Sakya Singha Sen

\title{
Preparation and Reactions of \\ Compounds with Heavier Group 14 \\ Elements in Low Oxidation States
}

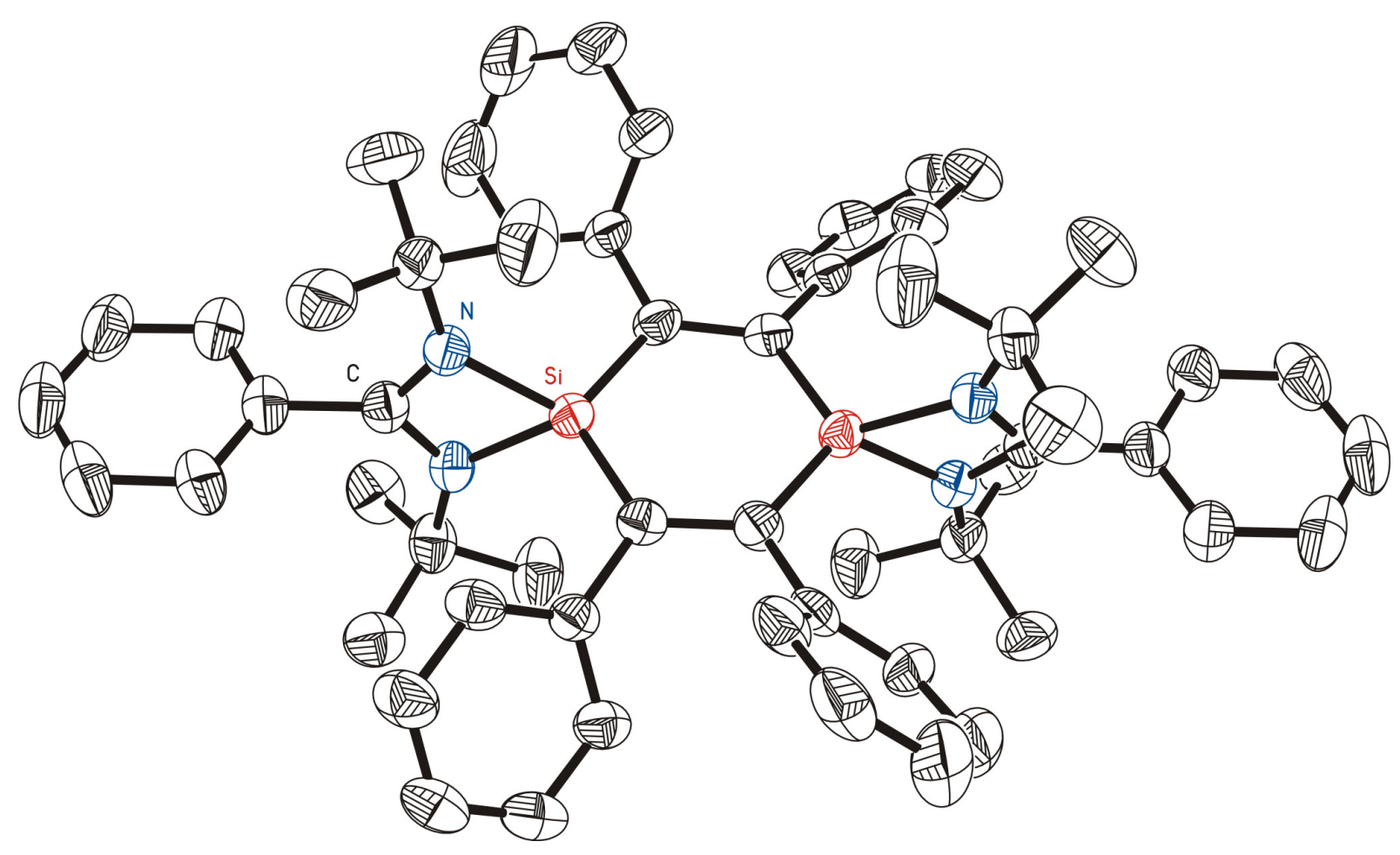

Göttingen 2010 


\title{
Preparation and Reactions of Compounds with
}

\section{Heavier Group 14 Elements in Low Oxidation States}

\author{
Dissertation \\ zur Erlangung des Doktorgrades \\ der Mathematisch-Naturwissenschaftlichen Fakultäten \\ der Georg-August-Universität zu Göttingen
}

vorgelegt von

Sakya Singha Sen

(aus Kolkata)

Indien

Göttingen 2010 
D 7

Referent: Prof. Dr. Dr. h. c. mult. Herbert W. Roesky

Koreferent: Prof. Dr. Dietmar Stalke

Tag der mündlichen Prüfung: 04.10.2010 
Dedicated to my parents for their love and affection 


\section{Acknowledgment}

The work described in this dissertation has been carried out under the guidance and supervision of Prof. Dr. Dr. h. c. mult. Herbert W. Roesky at the Institut für Anorganische Chemie der Georg-August-Universität in Göttingen between November 2006 and September 2010.

At first, I would like to express my gratitude to Prof. Herbert W. Roesky for giving an excellent working atmosphere, granting academic freedom and his continuous motivation to tackle the regular challenges in chemistry of group 14 elements. His enthusiasm for 'high quality results' and perseverance inspired me for not to give up till the end. His confidence that things will turn to work at the end definitely encouraged me to overcome a couple of experimental failures.

Furthermore, I would like to address my sincere thanks to my collaborators that added a great deal to the completion of my thesis: Prof. Dr. Dietmar Stalke not only for X-ray crystal structural investigations and theoretical calculations but for spending ample time for correcting my manuscripts for publication; Dr. Daniel Stern, Dr. Regine Herbst-Irmer, Prof. Carola Schulzke, Dr. Aritra Pal, Mr. Tobias Beck, Ms. Andrea Thorn, Mr. Daniel Kratzert, and Mr. Jakob Hey for dealing the X-ray diffraction experiments; Dr. A. Claudia Stückl for her enormous help in EPR measurement as well as handling the administration issues from my very first day in Göttingen; Dr. Julian Henn, Dr. Kathrin Meindl, Dr. Swapan K. Pati, Dr. Debashis Koley, Mr. Sudipta Dutta for their DFT calculation; Dr. Michael John, Mr. W. Zolke, Mr. R. Schöne for the assistance in all NMR-related concerns and Mr. T. Schuchardt for mass spectrometry. I am indebted to Mr. Martin Schlote and Mr. Matthias Hesse for their timely support during my experimental work. I am also very much grateful to the staff of the Analytical Laboratories, Electrical, and Glassblowing people for their apropos 
accompaniment. Deep thanks furthermore to all house-stewards and the cleaning service for maintaining the laboratory.

I would also like to thank to all my previous and present colleagues: Dr. Sanjay Singh, Dr. Cheuk -Wai So, Dr. Swadhin K. Mandal, Dr. Gregory Nikiforov, Dr. Victor Manuel JiménezPérez, Dr. Debojyoti Ghoshal, Dr. Sharanappa Nembenna, Dr. Prabhuodeyara Gurubasavraj, Dr. Zhi Yang, Dr. Sarish Sankar, Dr. Bijan Nekoueishahraki, Dr. Anukul Jana, Dr. Rajendra S. Ghadwal, Dr. Jianfeng Li, Dr. Ramachandran Azhakar, Dr. V. Arun, Dr. N. Dastagiri Reddy, Mr. Yang Ying, and Mr. Zhensheng Zhang. I would also like to acknowledge Mr. Malte P. Kritzler-Kosch for being involved in the experimental work with me.

At this point I would like to express my special thanks to Prof. Selvarajan Nagendran for helping me to teach how to work under inert atmosphere and also for numerous fruitful discussions. I am also grateful to Dr. Gaspar Tavcar and Dr. Shabana Khan for the way we jointly shared the topic- never as competitors, but always working hand in hand.

I express my best personal regards to Dr. Animesh Das, Mr. Vimal Nair, Mr. Prinson P. Samuel, Mr. Carlos Abad, Mr. Angshuman Mandal, Dr. Ashutosh Banerjee, Mr. Diptangshu Chakraborty, Dr. Akhilesh K. Singh, Mr. Gunther Speichert, and last but not the least Mr. Alexander Döring. I really consider myself fortunate to have them during my stay in Germany.

The financial support from the Göttinger Akademie der Wissenschaften and the Deutsche Forschungsgemeinschaft (DFG) is gratefully acknowledged.

Finally I would like to thank my parents and my family members for their motivation and support for accomplishing this work.
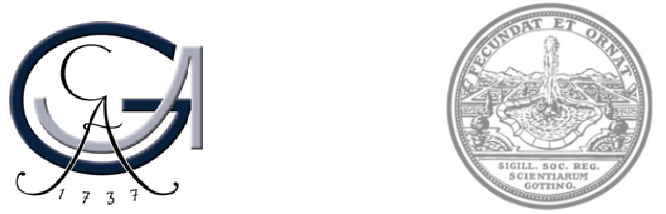


\section{Contents}

Chapter 1. General Introduction ..........................................................................................3

1.1 A brief history of low-valent group 14 elements ..................................................... 5

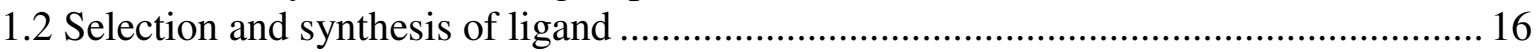

1.3 Focus and the content of this contribution .................................................................. 19

Chapter 2. Convenient and High Yield Access to Silylene and its Reactivity towards Unsaturated Compounds................................................................................................................ 22

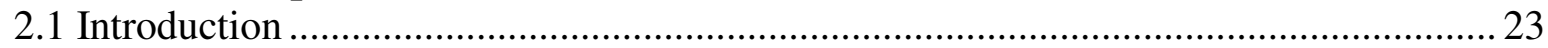

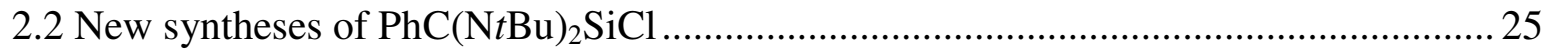

2.3 Reactivity of $\mathrm{LSiCl}\left(\mathrm{L}=\mathrm{PhC}(\mathrm{N} t \mathrm{Bu})_{2}\right)$ towards unsaturated organic compounds:

Synthesis of novel silicon containing ring compounds ...................................................26

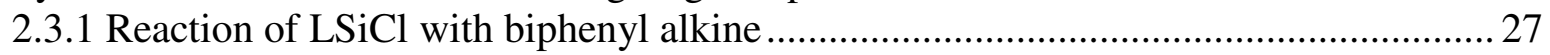

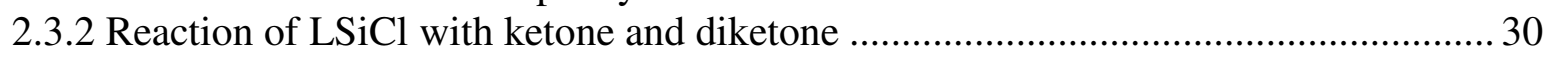

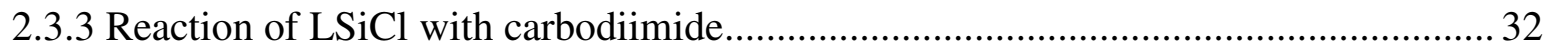

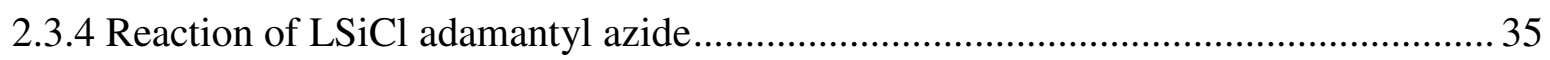

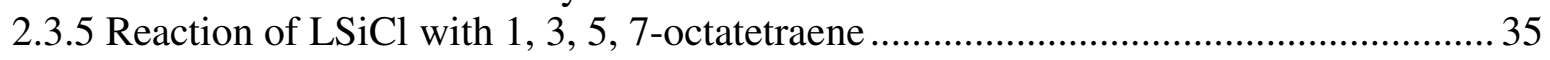

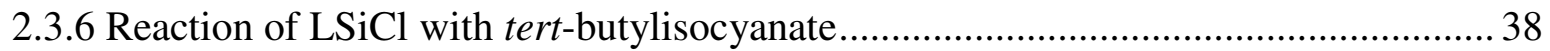

2.3.7 Reaction of $\mathrm{LSiCl}$ with trimethylamine $\mathrm{N}$-oxide ……..............................................4 40

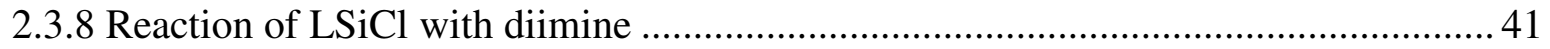

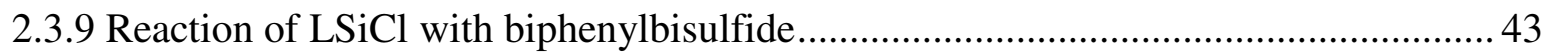

2.4.1 Reaction of $\mathrm{LSiCl}$ with adamantylphosphaalkine .................................................... 44

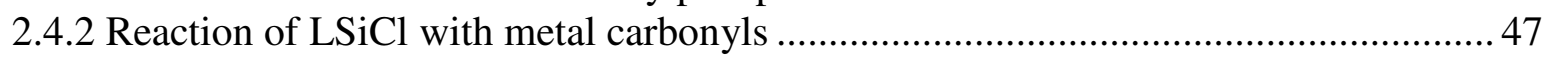

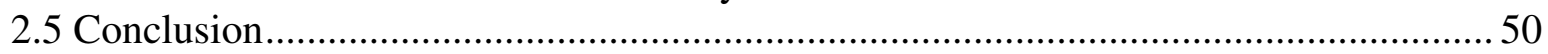

Chapter 3. A Remarkable Base Stabilized Bis-Silylene with a Si(I)-Si(I) Single Bond ...51

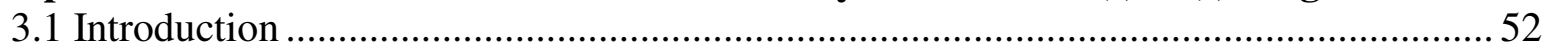

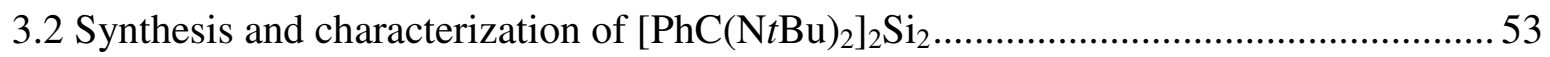

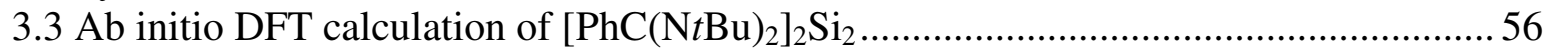

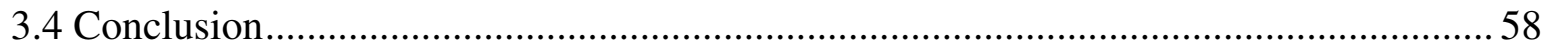

Chapter 4. Reactions of Base Stabilized Bis-Silylene $\left(\mathrm{PhC}\left(\mathrm{N} t \mathrm{Bu}_{2}\right)_{2} \mathrm{Si}_{2}\right)$ with various organic substrates: Probing the Nature of the Si-Si Bond .....................................................55

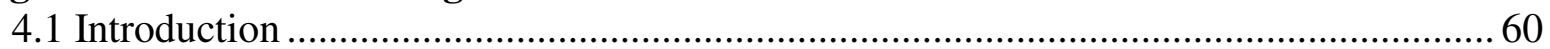

4.2. Reactions of bis-silylene with unsaturated organic compounds ................................... 61

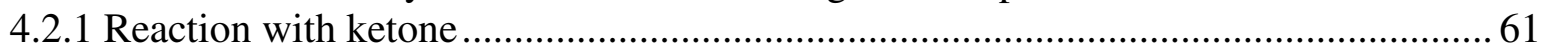

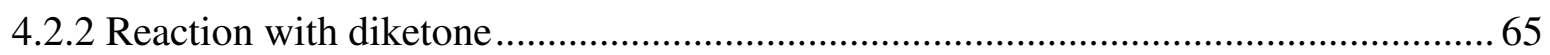

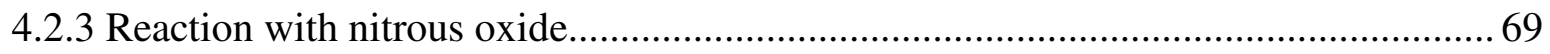

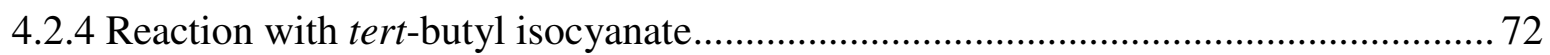

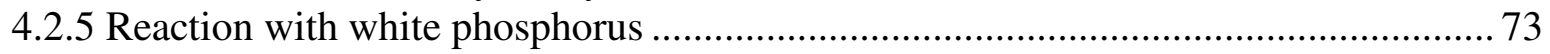

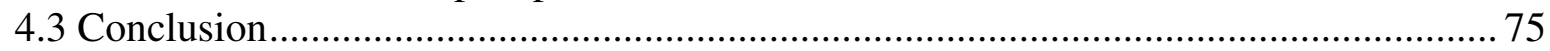

Chapter 5. Synthesis, Structure, and Theoretical Investigation of Amidinato Supported 1,4-disilabenzene.................................................................................................................76

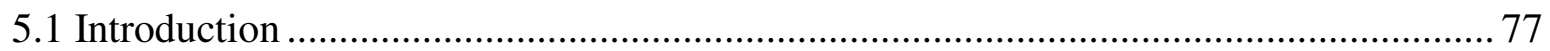

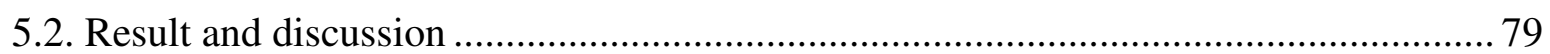

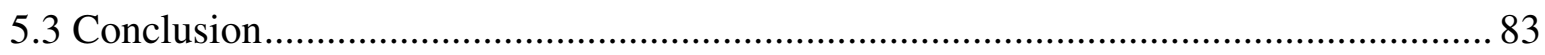

Chapter 6. Synthesis, Structural Elucidation, Theoretical Investigation and Reactivity

Studies of a Bis-germylene with a Ge(I)-Ge(I) bond ............................................................... 84

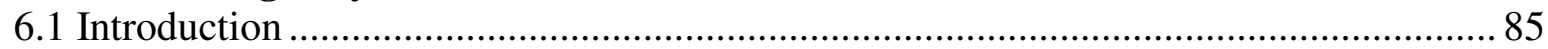

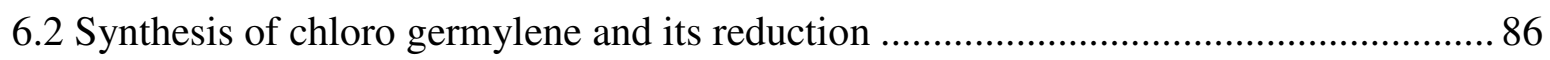

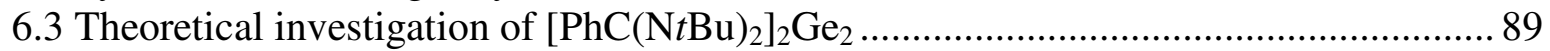

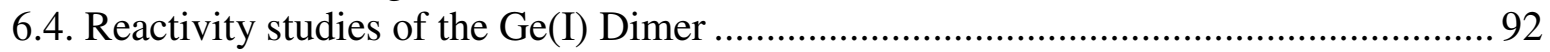




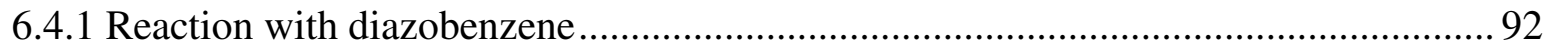

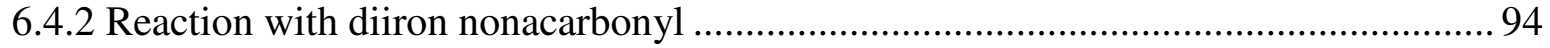

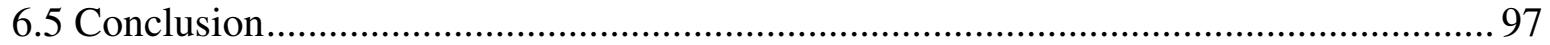

Chapter 7. Synthesis of Monomeric Divalent Tin(II) Compounds with Terminal

Chloride, Amide, and Triflate Substituents.................................................................................98

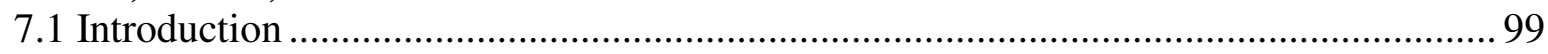

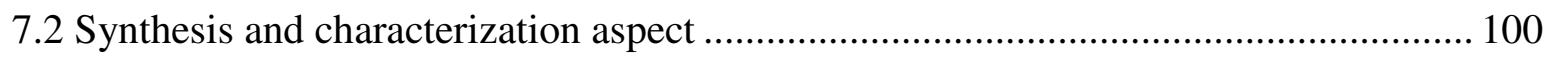

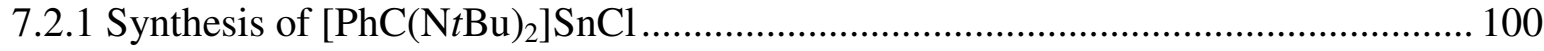

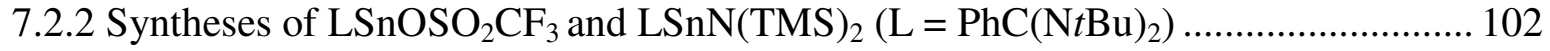

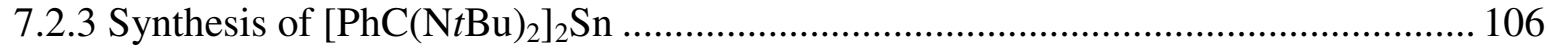

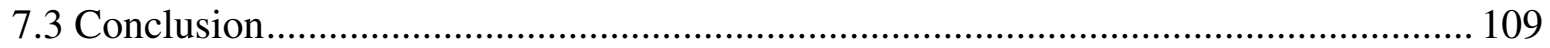

Chapter 8. Summary and Future Scope................................................................................... 110

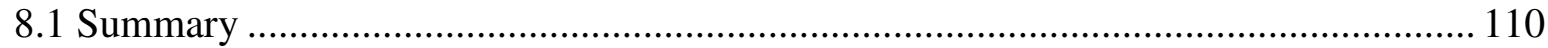

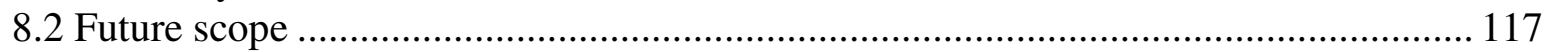

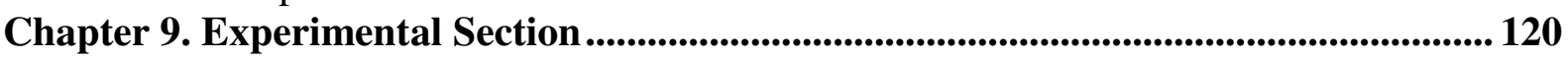

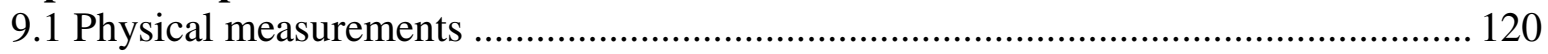

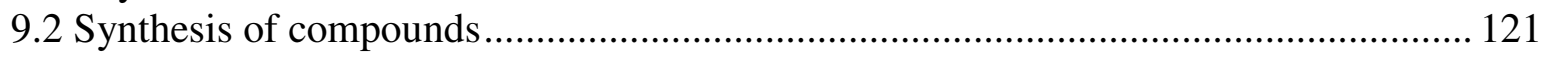

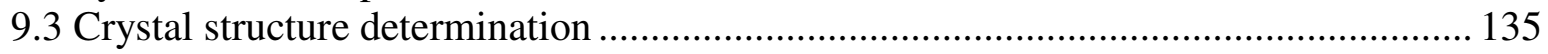

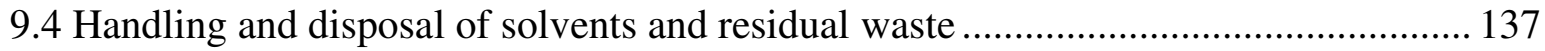

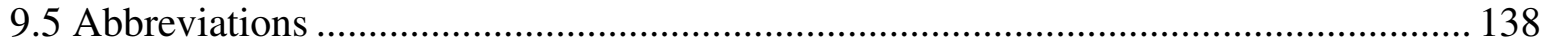

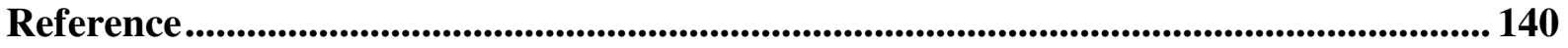




\section{Chapter 1}

\section{General Introduction}
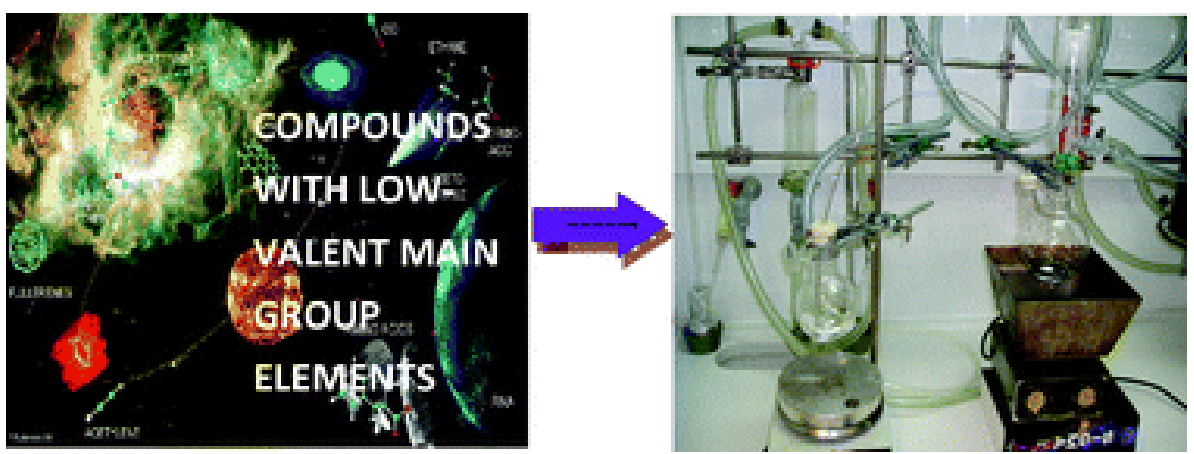

\section{Abstract}

The fundamental interest and formidable synthetic challenge regarding the synthesis of compounds with heavier low-valent group 14 elements is discussed and a general introduction covering a brief description of important compounds in this research area is provided. The selection and synthesis of ligand is vividly explained. The aim and the results presented in this contribution are outlined. 


\section{What the ocean is to the child, the Periodic Table is to the chemist}

K. Barry Sharpless (Nobel lecture, 2002)

Since the discovery of the periodic table by Mendeljeev and Lothar Meyer, it has become the part and parcel to a chemist's life because it provides a useful framework to classify, systematize, and compare all of the many different forms of chemical behavior. It makes chemistry more an art than a science. Every budding chemistry student eventually falls in love with at least one of the elements and/or groups of the periodic table. Although I am passionate about the whole periodic table, the elements in the group 14 particularly thrilled me from my school days. Beginning with the nonmetal carbon and concluding with lead, a malleable, poisonous heavy metal, the group 14 elements constitute an eclectic collection. Graciously residing between these two elemental extremes are the diverse group 14 elements silicon, germanium, and tin.

The organometallic chemistry of the main group has not developed in parallel with that of the transition metals although remarkable success has been achieved in the last two decades. A plausible explanation of this advancement may be due to the unprecedented reactivity of heavier group 14 elements. In a surprisingly brief period of time, organometallic compounds with low valent group 14 elements have blossomed at once into an engaging, intriguing, and still emerging area of chemistry. This introduction will draw a sharper attention to this rapidly expanding body of work. Carbene, silene, silylene, disilene, digermene, disiline, digermine, distannine, diplumbine, silaallene etc. are noteworthy additions to the diverse tapestry of organometallic chemistry. Even though these are not directly related with this dissertation, the author thinks it is apropos to acknowledge these groundbreaking syntheses concisely in the introduction of the dissertation. 


\subsection{A brief history of low-valent group 14 elements}

Carbenes are compounds in which the carbon atom is in a formal divalent state. ${ }^{1}$ Arduengo and co-workers isolated the first stable and structurally characterized N-heterocyclic carbene (NHC) (1) in $1991 .^{2}$ Compound $\mathbf{1}$ was obtained by reduction of 1,3-di-1-adamantylimidazol2-ylidene (Scheme 1). The key stabilizing effect in $\mathbf{1}$ is the donation of electron density from the nitrogen atoms into the empty $p$-orbital of carbon, which reduces its electrophilicity and increases its thermodynamic stability. By extending this stabilization principle, a large number of stable N-heterocyclic carbenes (NHCs) have been prepared. ${ }^{3}$ The NHCs have emerged as an important class of compounds due to their broad applications in organometallic chemistry, organic synthesis, and homogeneous catalysis. ${ }^{4}$

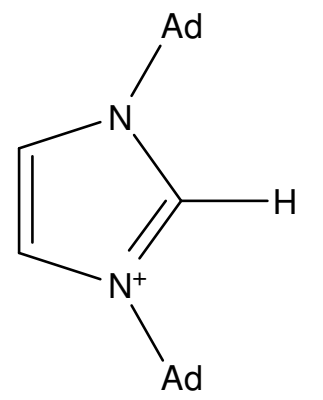

$\mathrm{NaH}, \mathrm{THF}$, cat. DMSO

$-\mathrm{NaCl},-\mathrm{H}_{2}$

$$
\text { Ad = Adamentyl }
$$

\section{Scheme 1.1. Synthesis of the first Stable N-heterocyclic carbene}

In view of this, much attention has also been paid to the heavier analogues of carbenes, i.e., silylenes $\left(\mathrm{R}_{2} \mathrm{Si}\right)$, germylenes $\left(\mathrm{R}_{2} \mathrm{Ge}:\right)$, stannylenes $\left(\mathrm{R}_{2} \mathrm{Sn}\right.$ :), and plumbylenes $\left(\mathrm{R}_{2} \mathrm{~Pb}\right.$ :). These so-called metallylenes are monomeric species of the polymetallanes. This is especially true for the silylenes, which are believed to be monomers of polysilane. The metallylenes could be expected to be of great importance in fundamental and applied chemistry as a result of their many differences and similarities to carbenes. The valency of the central atom of the heavier 
carbene analogues $\left(\mathrm{R}_{2} \mathrm{M}\right.$ :, $\left.\left.\mathrm{M}\right) \mathrm{Si}, \mathrm{Ge}, \mathrm{Sn}, \mathrm{Pb}\right)$ is two. Thus its formal oxidation state is $\mathrm{M}^{\mathrm{II}}$ and its stability increases as the principal quantum number $(n)$ increases. In fact, dichloroplumbylene $\left(\mathrm{PbCl}_{2}\right)$ and dichlorostannylene, $\left(\mathrm{SnCl}_{2}\right)$, are very stable ionic compounds. However, these dihalides exist as polymers or ion pairs both in solution and in the solid state. The dichlorogermylene complex $\mathrm{GeCl}_{2}$. dioxane ${ }^{5}$ is also known to be stable and isolable, whereas the dihalosilylenes are barely isolable compounds. ${ }^{6}$ Hence, the chemistry of the metallylenes has been considered mainly from the molecular chemistry point of view. ${ }^{7}$

\begin{tabular}{|c|c|c|c|c|c|}
\hline $\begin{array}{l}\text { Atom } \\
\mathrm{n}\end{array}$ & $\begin{array}{l}\mathrm{C} \\
2\end{array}$ & $\begin{array}{l}\mathrm{Si} \\
3\end{array}$ & $\begin{array}{c}\mathrm{Ge} \\
4\end{array}$ & $\begin{array}{c}\text { Sn } \\
5\end{array}$ & $\begin{array}{c}\mathrm{Pb} \\
6\end{array}$ \\
\hline \multicolumn{6}{|l|}{ Orbital Energy } \\
\hline ns & -19.39 & -14.84 & -15.52 & -13.88 & -15.41 \\
\hline $\mathrm{np}$ & -11.07 & -7.57 & -7.29 & -6.71 & -6.48 \\
\hline \multicolumn{6}{|l|}{ Ionization Energy } \\
\hline ns & 16.60 & 13.64 & 14.43 & 13.49 & 16.04 \\
\hline np & 11.26 & 8.15 & 7.90 & 7.39 & 7.53 \\
\hline Electron Affinity & 1.26 & 1.39 & 1.23 & 1.11 & 0.36 \\
\hline \multicolumn{6}{|l|}{ Electronegativity } \\
\hline Mulliken & 1.92 & 1.46 & 1.40 & 1.30 & 1.21 \\
\hline Pauling & 2.55 & 1.90 & 2.01 & 1.96 & 2.33 \\
\hline Allen & 2.28 & 1.76 & 1.81 & 1.68 & 1.91 \\
\hline \multicolumn{6}{|l|}{ Atomic Radius } \\
\hline ns & 1.58 & 2.20 & 2.19 & 2.48 & 2.39 \\
\hline np & 1.74 & 2.79 & 2.88 & 3.22 & 3.22 \\
\hline Polarizabilty & 1.76 & 5.38 & 6.07 & 7.7 & 6.8 \\
\hline
\end{tabular}

Table 1.1. Atomic Properties of Group 14 elements 
In table 1.1 we have shown the atomic properties of group 14 elements. In contrast to the carbon atom, the heavier group 14 atoms have a low ability to form hybrid orbitals. They therefore prefer the $(n s)^{2}(n p)^{2}$ valence electron configurations in their divalent species. ${ }^{8}$ Since two electrons remain as a singlet pair in the ns orbital, the ground state of $\mathrm{H}_{2} \mathrm{M}$ : (M) Si, Ge, $\mathrm{Sn}, \mathrm{Pb}$ ) is a singlet, unlike the case of $\mathrm{H}_{2} \mathrm{C}$;, where the ground state is a triplet (Figure 1.1).

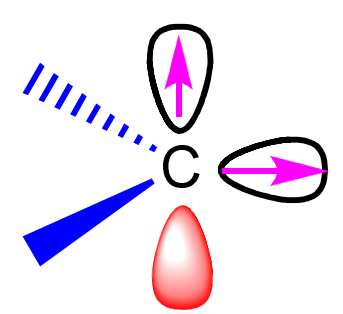

Triplet

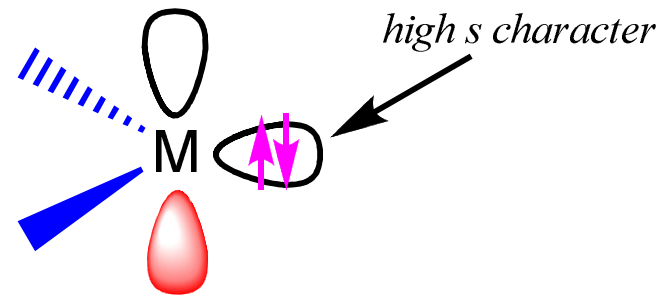

Singlet

$\mathrm{M}=\mathrm{Si}, \mathrm{Ge}, \mathrm{Sn}, \mathrm{Pb}$

Figure 1.1. Differences between the ground states of carbenes and metallyenes

On the basis of theoretical calculations, the singlet-triplet energy differences $\Delta E S T$ for $\mathrm{H}_{2} \mathrm{M}$, [ $\triangle E \mathrm{ST}) E$ (triplet) $-E($ singlet $)]$ are found to be $16.7(\mathrm{M}=\mathrm{Si}), 21.8(\mathrm{M}=\mathrm{Ge}), 24.8(\mathrm{M}=\mathrm{Sn})$, and $34.8(\mathrm{M}=\mathrm{Pb}) \mathrm{Kcal} / \mathrm{mol}$, whereas for $\mathrm{H}_{2} \mathrm{C}$ : it is estimated to be $-14.0 \mathrm{kcal} / \mathrm{mol}{ }^{9}$ Furthermore, the relative stabilities of the singlet species of $\mathrm{R}_{2} \mathrm{M}:(\mathrm{M}=\mathrm{C}, \mathrm{Si}, \mathrm{Ge}, \mathrm{Sn}, \mathrm{Pb} ; \mathrm{R}=$ alkyl or aryl) compared to the corresponding dimer, $\mathrm{R}_{2} \mathrm{M}=\mathrm{MR}_{2}$, are estimated to increase as the element row descends, $\mathrm{C}<\mathrm{Si}<\mathrm{Ge}<\mathrm{Sn}<\mathrm{Pb}$. It follows, therefore, that one can expect that a divalent organolead compound such as plumbylene should be isolable as a stable compound. 


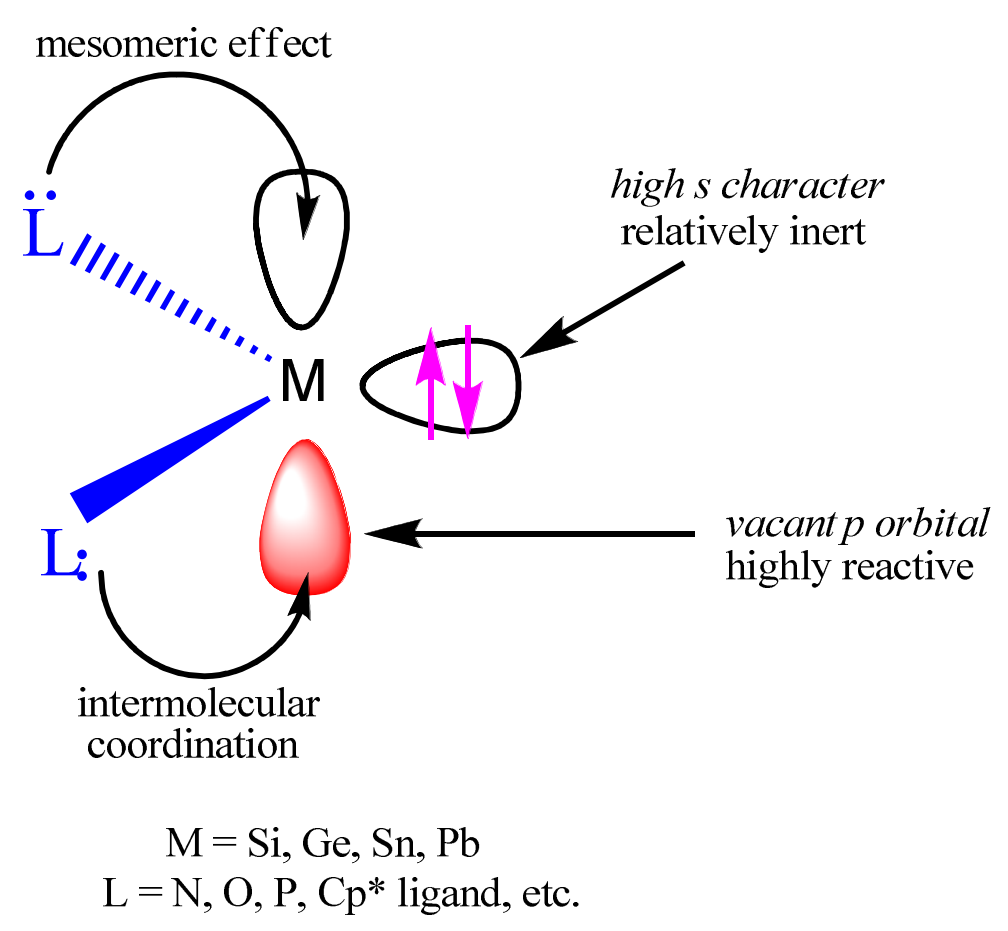

Figure 1.2. Thermodynamic stabilization of metallylenes

However, some plumbylenes without any electronic or steric stabilization effects are known to be thermally unstable and undergo facile disproportionation reactions, giving rise to elemental lead and the corresponding tetravalent organolead compounds. ${ }^{10}$ Metallylenes have a singlet ground state with a vacant $p$-orbital and a lone pair of valence orbitals. This extremely high reactivity must be due to their vacant $p$-orbitals, since six valence electrons is less than the eight electrons of the "octet rule". Their lone pair is expected to be inert due to its high $s$-character. On this basis, it can be inferred that it might be difficult to isolate metallylenes as stable compounds under ambient conditions, since they generally exhibit extremely high reactivity toward other molecules as well as themselves. In order to stabilize metallylenes enough to be isolated, either some thermodynamic and/or kinetic stabilization of the reactive vacant $p$-orbital is required (Figure 1.2). 
Nevertheless, by means of a tailor made starting material, West and co-workers in 1981 reported the isolation of a stable compound containing a silicon silicon double bond $\left(\mathrm{Mes}_{2} \mathrm{Si}=\mathrm{SiMes}_{2}\right)$ (2). ${ }^{11}$ Following this Brook et al. prepared the first silene [(TMS $\left.)_{2} \mathrm{Si}=\mathrm{C}(\mathrm{OTMS}) \mathrm{Ad}\right]$ (3) in the same year. ${ }^{12}$ The resulting paradigm shift led the burgeoning chemistry of the multiply bonded compounds of the heavier group 14 elements. Dozens of stable disilenes have been reported since the seminal synthesis of West's disilene. ${ }^{13}$ Following this, the first two structurally characterised stable digermenes $R_{2} G e=G_{2} R_{2}(R=$ 2,6- $\mathrm{Et}_{2} \mathrm{C}_{6} \mathrm{H}_{3}(\mathbf{4})^{14}$ and $\mathrm{CH}(\mathrm{TMS})_{2}(\mathbf{5})^{15}$ ) were reported in 1984 by the Masamune and Lappert groups independently. In contrast to the carbon system, double bonds containing heavier Group 14 elements have inherent high trans-bent structure. The unique structural feature has been investigated by theoretical study.<smiles>C[Si](C)=[Si](C)[Si](C)(C)C</smiles><smiles>Cc1cc(C)cc(C)c1</smiles>
2 West 1981

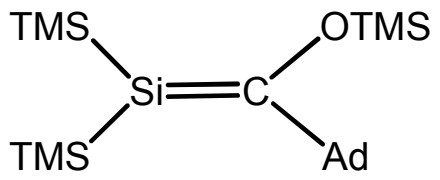<smiles>[Ge]=C1C2CC3CC(C2)CC1C3</smiles>

3 Brook 1981<smiles>CCc1cccc(CC)c1</smiles>

Masamune 1984

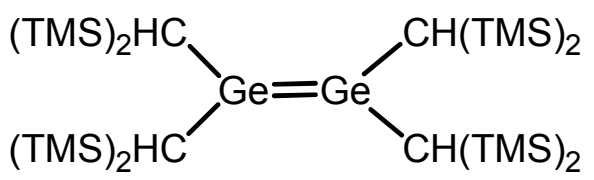

5

Lappert 1984

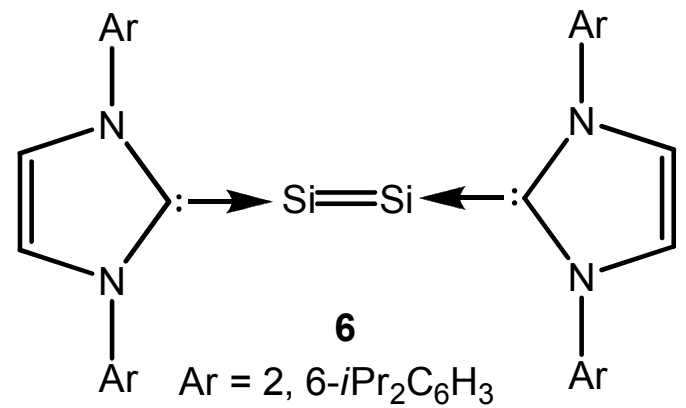

Robinson 2008

Chart 1.1. Diagrams for the notable double-bonded species containing heavier group 14 atoms 
A conceptual approach to rationalizing pyramidal or bent geometries in the double bond system of the heavier Group 14 elements begins with the recognition of the electronic structure of the molecular fragments. Carbene $\left(\mathrm{R}_{2} \mathrm{C}\right.$ : $)$ possesses a triplet ground state, so that two carbenes dimerize in a manner to form a planar structure of alkene $\left(\mathrm{R}_{2} \mathrm{C}=\mathrm{CR}_{2}\right)$. Meanwhile, the ground state of heavier Group 14 element carbene analogues is singlet. Bringing these singlet monomers together to dimerize them results in considerable repulsion between the lone pairs. This repulsion can be minimized by trans-bent geometry of double bond resulting from the donor-acceptor interactions between the occupied $n$ s orbitals and the empty $n \mathrm{p}$ orbitals of two singlet monomers. Then interaction occurs to bind the two monomers strongly affording the trans-bent structure. The interaction to form the trans-bent structure can be explained by molecular orbital mixing under distortion from the planar geometry. Under pyramidalization of the metal center, the E-E $\sigma^{*}$ orbital of the $\mathrm{E}_{2} \mathrm{H}_{4}$ system will mix into the $\pi$ orbital (HOMO), leading to further stabilization of the latter orbital. On descending the group, the degree of mixing, and hence the degree of energy lowering increases in order to reduce the energy gap between these orbitals. 

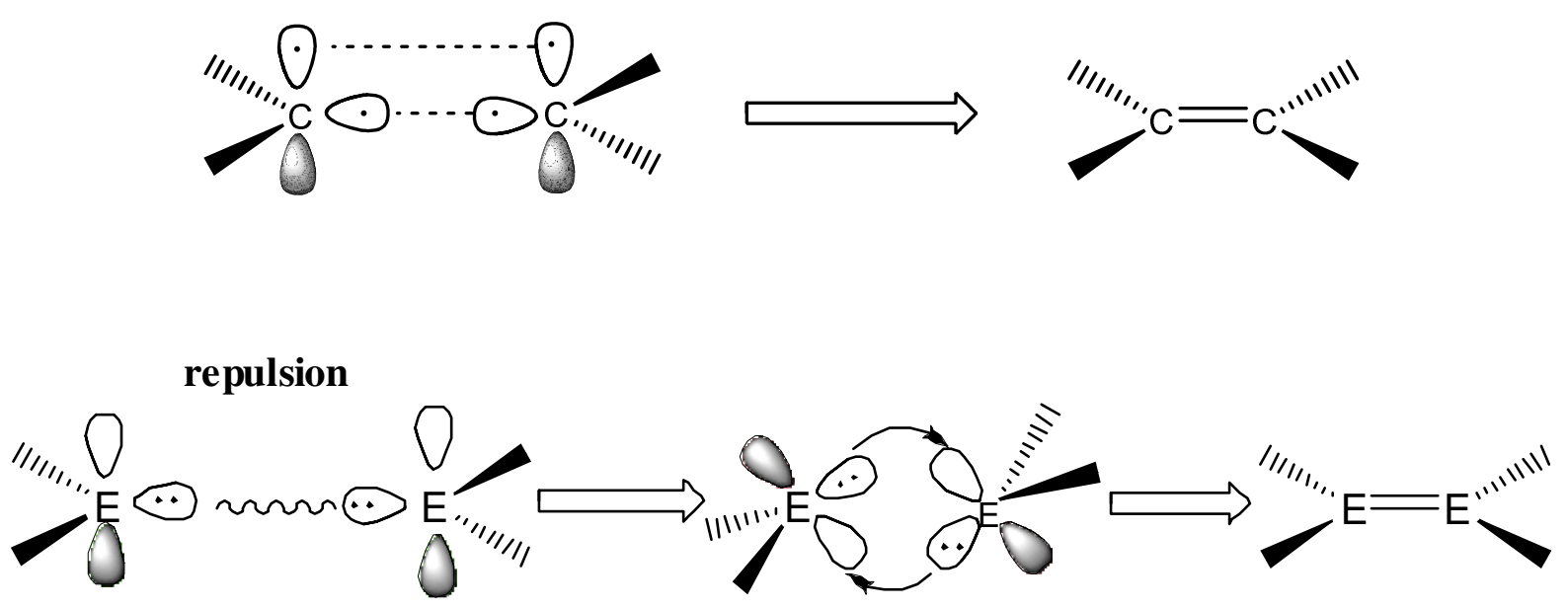

$\sigma^{*}-\pi$ interaction
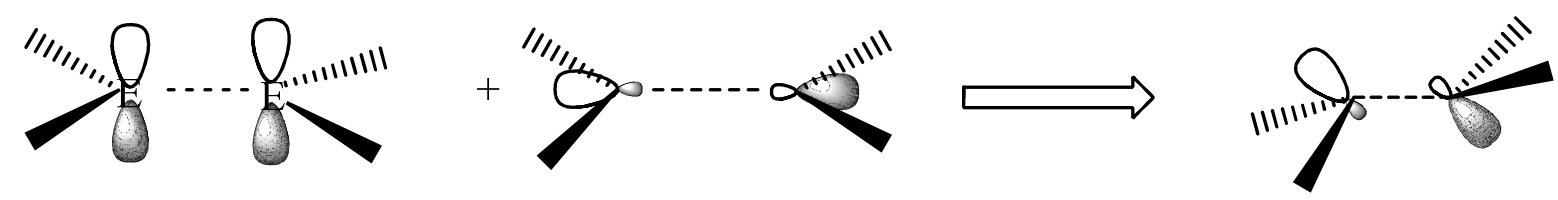

Figure 1.3. Schematic explanation for formation of trans-bent geometry

When the question of double bond formation between the heavier elements was almost largely settled, attention turned to triple bonds. An epoch making result in the chemistry of stable heavier analogue of alkine came in 2000 when first alkine analogue of lead, namely an amber-green diplumbine (vide infra) $\left[\mathrm{RPbPbR} ; \mathrm{R}=2,6-\mathrm{Trip}_{2} \mathrm{C}_{6} \mathrm{H}_{3}\right.$ (Trip = 2,4,6-i $\mathrm{Pr}_{3} \mathrm{C}_{6} \mathrm{H}_{2}$ ) (7) was isolated by Power and coworkers. ${ }^{16}$ Following this, the first carmine red-orange digermine $\left[\mathrm{RGeGeR} ; \mathrm{R}=2,6-\mathrm{Dipp}_{2} \mathrm{C}_{6} \mathrm{H}_{3}\right](\mathbf{8})^{17}$ and dark blue-green distannine $[\mathrm{RSnSnR} ; \mathrm{R}=$ 2,6-Dipp $\left.{ }_{2} \mathrm{C}_{6} \mathrm{H}_{3}\left(\mathrm{Dipp}=2,6-i \mathrm{Pr}_{2} \mathrm{C}_{6} \mathrm{H}_{3}\right)\right](9)^{18}$ were also reported by the same group in 2002 . However, all this alkine analogue of group 14 elements show marked lone pair character and decreasing $\pi$ - overlap of the bonded atoms, thus reducing the bond order from 3 (a triple bond) to 2 (a double bond) for germanium and tin and 1 (a single bond) for lead. The series was completed in 2004 with the first isolation of an emerald-green disilyne [RSiSiR; R = $i \operatorname{Pr}\left\{\mathrm{CH}\left(\mathrm{SiMe}_{3}\right)_{2}\right\}_{2}(\mathbf{1 0})$ by Sekiguchi and coworkers. ${ }^{19}$ In sharp contrast to alkine, which is 
linear it adopted a trans-bent geometry around the $\mathrm{Si}-\mathrm{Si}$ triple bond with the $\mathrm{Si}=\mathrm{Si}-\mathrm{Si}$ bond angle of $137.44^{\circ}$. Following this a few other alkine analogues have been reported till to date, ${ }^{20}$ which will be mentioned in the introductory paragraph of respective chapters. But what is a true landmark in the main group element multiple bond chemistry is the isolation of another disilene $\mathrm{RSi}=\mathrm{SiR},\left[\mathrm{R}=1,3\right.$-bis- $\left(2,6-i \mathrm{Pr}_{2} \mathrm{C}_{6} \mathrm{H}_{3}\right)$ imidazol-2-ylidene $\left.)\right](\mathbf{6})^{21}$ where the formal oxidation state of silicon is zero. The oxidation state of silicon can be assessed on the basis of the bond angles and distances. The X-ray crystal structure reveals a distinctly nonlinear C-SiSi-C backbone (C-Si-Si angle of $\left.93.37^{\circ}\right)$ and long $\mathrm{C}-\mathrm{Si}$ bonds $(1.927 \AA$ ). Thus the carbene ligand does not withdraw electron density from silicon but leaves a nonbonding electron pair on each silicon center.

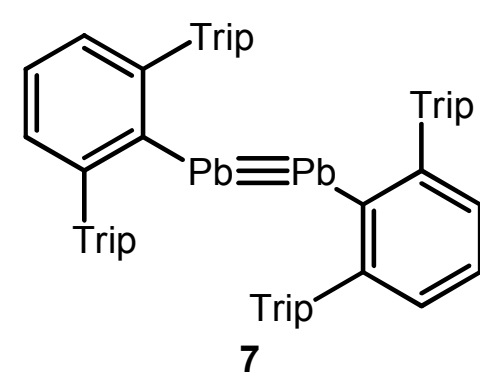

Trip $=2,4,6-i \mathrm{Pr}_{3} \mathrm{C}_{6} \mathrm{H}_{2}$

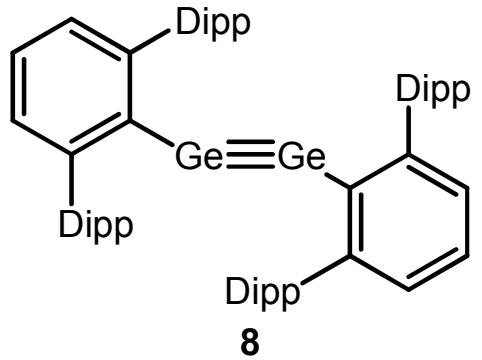

Dipp $=2,6-i \mathrm{Pr}_{2} \mathrm{C}_{6} \mathrm{H}_{3}$

Power (2002)<smiles>O=C(c1ccccc1)c1cccc([Pb])c1[Se]#Cc1c(P)cccc1[Pb]</smiles>

Dipp $=2,6-i \mathrm{Pr}_{2} \mathrm{C}_{6} \mathrm{H}_{3}$

Power (2000)

Power (2002)

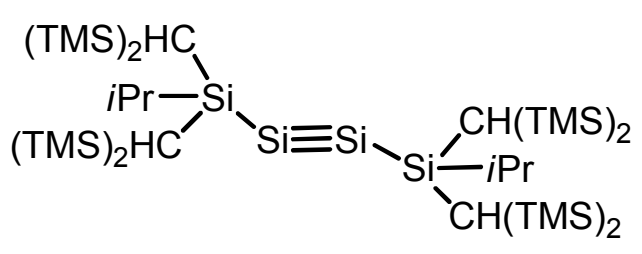

10

Sekiguchi (2004)<smiles>[Ge]c1cc(Br)c([Ge]#[Ge])c([Hg])c1</smiles>

11

$\mathrm{Tsi}=\mathrm{C}(\mathrm{TMS})_{2}$

Tokitoh (2006)

Chart 1.2. Alkine analogue of heavier group 14 elements 
Apart from multiply bonded group 14 compounds much attention was paid to the typical carbene analogue of silicon, silylene. Silylenes are divalent silicon compounds and normally have a singlet ground state $\left({ }^{1} \mathrm{~A}_{1}\right)$ due to the large energy gap between the $3 s$ and $3 p$ orbitals of the silicon atom. In organosilicon chemistry they are key intermediates in various thermal and photochemical reactions. They are very reactive, and most of them decompose immediately at temperatures above $77 \mathrm{~K}$. As reaction intermediates, the silylenes are even more important in silicon chemistry than their counterparts, the carbenes, are in organic chemistry. In 1994 West et al. pioneered the track by isolating the first stable and structurally characterized dicoordinate $\mathrm{N}$-heterocyclic silylene $\left[(\mathrm{CHN} t \mathrm{Bu})_{2} \mathrm{Si}\right](\mathbf{1 2})^{22}$ which is analogous to $\mathrm{N}$ heterocyclic carbene 1. The successful isolation of stable silylene by West completed for the silylenes a classic progression, from transient intermediate to matrix-isolated molecule to stable compound. Since then, the field of stable silylene research has become subject of extensive studies and by means of tailor-made synthetic strategies that utilize ligands with exact steric and electronic balance, a fair number of room temperature stable silylene complexes were synthesized and structurally characterized. ${ }^{23}$ The preparation of $\mathbf{1 2}$ was followed by the synthesis of its saturated analogue 13, also by West and co-workers, the preparation of the benzo-fused silylenes $\mathbf{1 4 a}$ and $\mathbf{1 4 b}$ by the Lappert-Gehrhus team, and the isolation of the pyrido-fused analogue 15 by Heinicke and co-workers. Following this, Kira and co-workers have isolated a silylene $\mathbf{1 6}$, with no such nitrogen stabilization. Very recently Driess et al. synthesized N-heterocyclic silylene 17. Of these known isolable silylenes 12, 14a, 14b, 15, and 17 are indefinitely stable, but 13 and 16 are only marginally stable. 
<smiles>CC(C)(C)N1C=CN(C(C)(C)C)[Si]1</smiles>

12

West (1994)<smiles>CC(C)(C)N1CCN(C(C)(C)C)[Si]1</smiles>

13

West (1996)<smiles>[R]c1ccc2c(c1)N([N+]([O-])[O-])[Si]N2[N+]</smiles>

14a $\mathrm{R}=\mathrm{H}$

14b $\mathrm{R}=\mathrm{Me}$ $\mathrm{Np}=$ Neopentyl

Lappert (1995)<smiles>[N+]N1[Si]N([N+](=O)[O-])c2cccnc21</smiles>

15

Heinicke (1998)

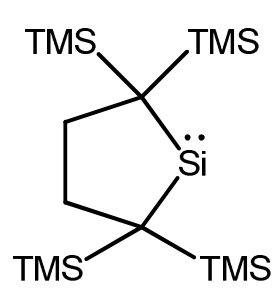

16

Kira (1999)

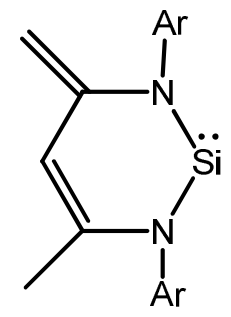

17 , $\mathrm{Ar}=2,6-$ ${ }^{2} \mathrm{Pr}_{2} \mathrm{C}_{6} \mathrm{H}_{3}$

Driess (2006)

Chart 1.3. Diagrams for the silylene $12-17$.

Surprisingly very little attention was paid to the synthesis of chloro silylenes, although they can be considered as analogues of gaseous silicon dichloride. It is also well known that silylene complexes with small substituents like $\mathrm{Cl}, \mathrm{Me}, \mathrm{Ph}$ are considered as reactive intermediates. ${ }^{24}$ Therefore, the isolation of these types of silylene complexes is a synthetic challenge. Recently we pursued such targets and successfully reported the synthesis of tricoordinate heteroleptic chloro silylene $\left[\mathrm{Ph}(\mathrm{N} t \mathrm{Bu})_{2} \mathrm{SiCl}\right](\mathbf{1 8})$ and carbene stabilized dichloro silylene ( $\mathrm{RSiCl}_{2}, \mathrm{R}=1,3$-bis- $\left(2,6-i \mathrm{Pr}_{2} \mathrm{C}_{6} \mathrm{H}_{3}\right)$ imidazol-2-ylidene) (19) ${ }^{25}$ These two compounds, particularly the latter one would be considered as a model compound for the silicon dichloride $\left(\mathrm{SiCl}_{2}\right),{ }^{26}$ which is unstable at room temperature.<smiles></smiles>

18

Roesky (2006)<smiles>Cl[Si](Cl)(Cl)C[C]1N([Ga])C=CN1Br</smiles>

19, $\mathrm{Ar}=2,6-\mathrm{Pr}_{2} \mathrm{C}_{6} \mathrm{H}_{3}$

Roesky (2009)

Chart 1.4. Diagrams of the chloro silylenes 18 and 19. 
Insofar the synthesis and isolation of important compounds containing low valent heavier group 14 elements is described in a nutshell. The most dramatic structural feature of these compounds containing homonuclear multiple bonds of the heavier main group elements is the trans-bent geometry. The donor-acceptor bonding mode may be used to rationalize the transbent geometry of the multiple bonds of group 14 elements. This bonding mode is also relevant in understanding some counterintuitive bonding phenomenon that is often observed (i.e. the $\mathrm{Pb}=\mathrm{Pb}$ double bond is even longer than the $\mathrm{Pb}-\mathrm{Pb}$ single bond). ${ }^{27}$ Indeed, given the preponderance of decidedly "non-carbon" like behavior of group 14 elements Prof. Yitzhak Apeloig rightly said "The heavier main-group elements, Si to Pb, actually exhibit 'normal' chemical behavior while carbon should be considered the 'unusual' member of group 14 elements rather than the prototype".

But in spite of all these breakthroughs it would not be an overstatement that the lowvalent main group chemistry is still a field in its infancy and presents many challenges for the future. For example a stable silyne $(-\mathrm{Si} \equiv \mathrm{C}-)$, sila-nitrile $(\mathrm{Si} \equiv \mathrm{N}), \mathrm{Si} \equiv \mathrm{P}$, and bis carbene with $\mathrm{C}(\mathrm{I})-\mathrm{C}(\mathrm{I})$ bond are still unknown. So to obtain a better understanding of the principles and rules, which lie behind the results in details we are motivated to probe the subvalent chemistry of heavier group 14 elements and compare their structures, bonding and reactivity with fundamental organic compounds like carbene, and alkine. 


\subsection{Selection and synthesis of ligand}

Everything has its science, with the exception of catching fleas: This is an art.

Dutch Proverb

The selection of a suitable ligand is indeed an art in main group chemistry because the utilization of bulky ligands, with appropriate steric and electronic effects, is a crucial factor in the stabilization of compounds with low valent and low coordinate group 14 elements. Several ligands were already proved to be very efficient in this perspective. In the next page we listed some ligands, which are widely used by different working groups for stabilizing compounds with low valent elements. For example our group has already developed the chemistry of $\mathrm{Al}(\mathrm{I}), \mathrm{Ge}(\mathrm{II})$ and $\mathrm{Sn}(\mathrm{II})$ with the support of sterically hindered $\beta$-diketiminato ligand $\mathrm{HC}(\mathrm{CMeNAr})_{2}\left(\mathrm{Ar}=2,6-i \mathrm{Pr}_{2} \mathrm{C}_{6} \mathrm{H}_{3}\right) .{ }^{28}$ Recently Driess et al. also stabilized silylene with the support of this ligand. ${ }^{23 c}$ Jutzi and co-workers showed $\mathrm{Cp}^{*}$ is efficient in stabilizing decamethyl silicocene, the ferrocene analogue of silicon. ${ }^{29 a}$ The same ligand was used later by Carmona et al. during the epoch-making synthesis of $\mathrm{Zn}(\mathrm{I}) .{ }^{29 \mathrm{~b}}$ Power and his team employed the terphenyl ligand system and were able to isolate a series of alkine analogues of heavier group 14 elements. ${ }^{16-18}$ Moreover, they were even successful in isolating a compound with a $\mathrm{Cr}-\mathrm{Cr}$ quintiple bond using the same ligand system. ${ }^{30}$ Recently Jones et al. reported the remarkable synthesis of first $\mathrm{Mg}(\mathrm{I})$ dimer with the support of guanidinato ligand. Moreover they prepared another derivative of $\operatorname{Mg}(\mathrm{I})$ dimer using $\beta$-diketiminato ligand $\mathrm{HC}(\mathrm{CMeNAr})_{2} \cdot{ }^{31}$ The stability and strong electron-pair donor capabilities of N-heterocyclic carbenes have been well documented. Robinson et al. utilizing these unique properties of Nheterocyclic carbenes recently synthesized and structurally characterized alkene analogues of boron, ${ }^{32 \mathrm{a}, 32 \mathrm{~b}}$ silicon, ${ }^{21}$ phosphorus, ${ }^{32 \mathrm{c}}$ arsenic, ${ }^{32 \mathrm{~d}}$ and gallium. ${ }^{32 \mathrm{e}}$ 


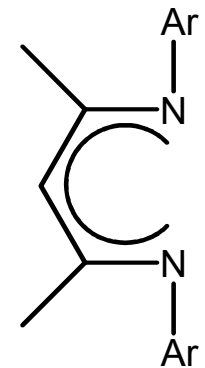

B-Diketiminato ligand; $\mathrm{Ar}=2,6-i \mathrm{Pr}_{2} \mathrm{C}_{6} \mathrm{H}_{3}$<smiles>[R2]C(=NBr)NBr</smiles>

Guanidinato ligand; $\mathrm{Ar}$ $=2,6-i \mathrm{Pr}_{2} \mathrm{C}_{6} \mathrm{H}_{3} ; \mathrm{R}=$ cyclohexyl/ $t \mathrm{Bu}$<smiles>BrN1C=CN(Br)C1</smiles>

$\mathrm{N}$-heterocyclic

carbene; $\mathrm{Ar}=2,6$ $i \mathrm{Pr}_{2} \mathrm{C}_{6} \mathrm{H}_{3}$

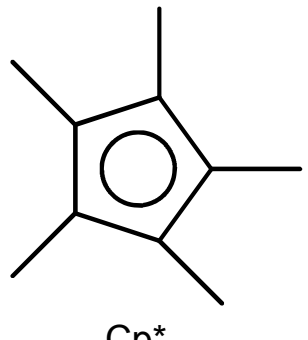

$\mathrm{Cp}{ }^{*}$

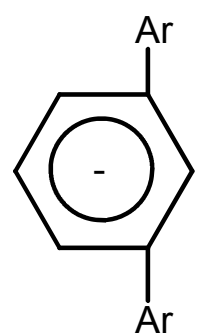

Terphenyl ligand; $\mathrm{Ar}=2,4,6-i \mathrm{Pr}_{3} \mathrm{C}_{6} \mathrm{H}_{2}$

Chart 1.5. Different ligand systems utilized by contemporary working groups

In view of these literature surveys we decided to stabilize a ligand system, which is not very widely used in main group chemistry for stabilization of low oxidation states of group 14 elements. In order to explore such chemistry of three-coordinate group 14 compounds, it was necessary to design a ligand with the following properties: (1) easy to synthesize and modify, (2) coordinate strongly to metal preferably as a bidentate ligand, (3) provide the opportunity to fine-tune the ligand by altering substituents, (4) discourage the propensity of formation of homoleptic compounds. A ligand that fits into these criteria is the four-membered monoanionic amidinato ligand. Amidines were first synthesized by Gerhardt in 1858 by the reaction of aniline with $N$-phenylbenzimidyl chloride and were named after the acid or amide obtained from it after hydrolysis. ${ }^{33}$ Thus, when $\mathrm{R}^{\prime}=\mathrm{H}$, the compound is known as a formamidine; $\mathrm{R}^{\prime}=\mathrm{CH}_{3}$, acetamidine; $\mathrm{R}^{\prime}=\mathrm{Ph}$, benzamidine. Amidines act as two-electron donors via the more basic and less sterically crowded imino lone pair, to form simple adducts such as $\mathrm{CoCl}_{2}(\mathrm{AmH})_{2}$ and $\mathrm{PdCl}_{2}(\mathrm{AmH})_{2}$, where $\mathrm{AmH}=$ amidine, but a more interesting group 
is formed when the $\mathrm{R}^{4}$ group is displaced to form the amidinato group, $\mathrm{R}^{2} \mathrm{NC}\left(\mathrm{R}^{\prime}\right) \mathrm{NR}^{3}$, which is isoelectronic with triazenato, carboxylato, sulfurdiimino etc.

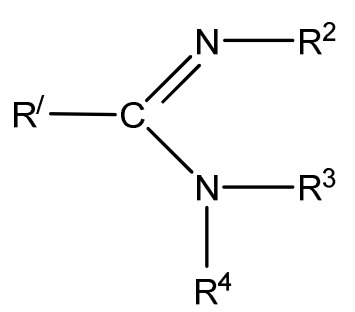

Figure 1.4. The general structure of an amidine

The major advantage of using amidinato ligands is that their steric and electronic properties are readily modified through variation of the substituents on the carbon and nitrogen atoms. Due to the geometric constraints of the NCN ligand backbone, amidinates have small N-M-N bite angles (typically $63-65^{\circ}$ ). They have a rich coordination geometry in which both chelating and bridging coordination modes can be achieved. The balance between chelating and bridging coordination is critically governed by the substitution pattern of the amidinate ligand. Large substituents on the carbon atom induce a convergent orientation of the lone pairs (favoring chelation), while small substituents lead to a more parallel orientation of the lone pairs (enabling bridging). ${ }^{34}$ The substituents on the amidinato nitrogen atoms can be used for tuning the steric requirement of the ligand, that influences the coordination geometry of the metal center. The 2,6-i $\operatorname{Pr}_{2} \mathrm{C}_{6} \mathrm{H}_{3}$ group has turned out to be very efficient in that respect, and has been used very recently in the development of stabilization of low valent germanium by Jones' working group. We also had initial success to stabilize first heteroleptic chloro silylene (18) by the support of amidinato ligand using $t \mathrm{Bu}$ substituents on nitrogen atoms. Benzamidinate type complexes of nickel, vanadium, and yttrium have already been probed for catalytic reactivity, and it has been found that the ligand is attractive towards catalysis. This may be due to the fact that it offers a similar level of steric protection to that of the 
cyclopentadienyl group but affords greater electrophilicity on the metal center as a consequence of being only four-electron donor and the prodigious size to access the threecoordinate group 14 metals. So we selected amidinato ligand (20) judiciously and probed whether it can stabilize subvalent group 14 elements. The synthetic procedure of $\mathbf{2 0}$ was very straightforward. The reaction of tert-butylcarbodiimide with one equivalent of $\mathrm{PhLi}$ in diethyl ether afforded 20 (Scheme 1.2). Our findings pertaining to $\mathrm{Si}(\mathrm{II}), \mathrm{Si}(\mathrm{I}), \mathrm{Ge}(\mathrm{II}), \mathrm{Ge}(\mathrm{I})$, and $\mathrm{Sn}(\mathrm{II})$ chemistry with the support of benzamidinato ligand are vividly discussed in this thesis.

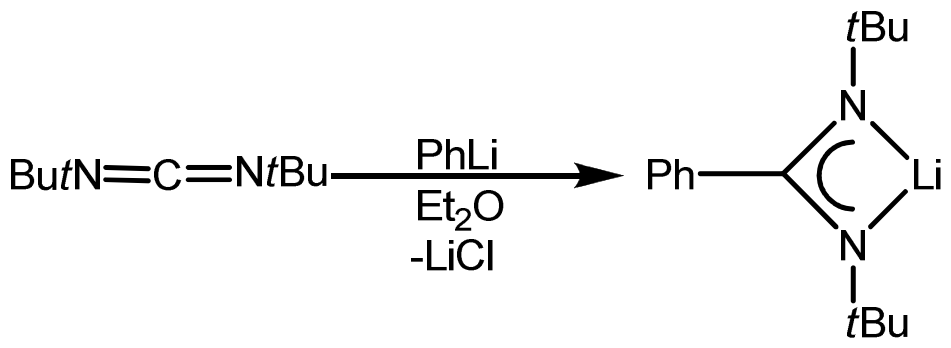

20

Scheme 1.2. Preparation of amidinato lithium

\subsection{Focus and the content of this contribution}

It is hoped that the introduction has accounted that the heavier analogues of carbene are no longer imaginary species, nor reactive intermediates, but they are "bottleable" when appropriately stabilized by kinetic and/or thermodynamic methods. Their unprecedented structures, novel bonding arrangements, and unusual patterns of reactivity continue to thrive. Despite these impressive progresses it is clear from the paucity of compounds with low valent group 14 elements that many more exciting discoveries remain to be made. Besides there is some recent development in low valent main group chemistry driven by the demands of material science and medical science. Moreover there is also an ongoing quest for improved catalysts and cocatalysts that can be used for effecting industrially important chemical transformations. Compounds with low valent main group elements have considerable potential as catalysts or reagents for chemical synthesis. ${ }^{35}$ Finally, there is a huge interest in 
recent days about interstellar and circumstellar compounds. ${ }^{36}$ It is believed that the compounds with low valent atoms exist there due to the absence of intermolecular collision. For example N-heterocyclic carbene which was isolated in laboratory by Arduengo et al. ${ }^{2}$ is detected explicitly in interstellar space. ${ }^{36}$ We hope that our synthesis of compounds with low valent group 14 elements under laboratory condition, their characterizaion, structural elucidation, and theoretical calculation will be useful for interstellar detection of them.

Chemistry provides not only the mental discipline, but an adventure and an aesthetic experience

\section{Cyril Hinshelwood}

Keeping the remark of Hinshelwood in mind we embarked on the adventure and the aesthetic experience of studying the chemistry of low oxidation state of heavier group 14 elements. Synthesis and characterization of new group 14 metallylenes with the support of benzamidinato ligand and investigation of their reactivities is the focus of this thesis. To achieve this goal we have encountered several exciting phenomena, which are documented in this thesis. All the compounds obtained were fully characterized by NMR spectroscopy, EIMS spectrometry, elemental analysis, and single crystal X-ray diffraction. In this part of the thesis we will give a very small description of the content of each chapters of the thesis.

Chapter 2 presents the two new approaches to synthesize previously reported heteropleptic chlorosilylene in excellent yield, which were not known in the literature before. Furthermore, the tri-coordinate silylene was reacted with unsaturated compounds to afford different kinds of small ring silicon compounds. All these reactivities along with complete characterization data are summerized in this chapter. 
Chapter 3 contains the synthesis, structure and theoretical calculation of a compound consisting of a $\mathrm{Si}(\mathrm{I})-\mathrm{Si}(\mathrm{I})$ bond along with a lone pair of electrons on each silicon atom. This is a very rare compound where the $\mathrm{Si}-\mathrm{Si}$ single bond is stabilized by a monoanionic benzamidinato ligand and avoids the lone pair of electrons taking part in any bonding.

Chapter 4 describes the reactivity of the $\operatorname{Si}(\mathrm{I})-\mathrm{Si}(\mathrm{I})$ containing compound with various organic substrates such as ketone, diketone, $\mathrm{N}_{2} \mathrm{O}$, tert-butyl isocyanate, white phosphorus. With the help of these reactivities we successfully correlated the molecular structure of $\mathrm{Si}(\mathrm{I})$ $\mathrm{Si}(\mathrm{I})$ and its bonding arrangements.

Chapter 5 presents the first isolable 1,4-disilabenzene obtained by the reaction of $\mathrm{Si}(\mathrm{I})-\mathrm{Si}(\mathrm{I})$ with biphenyl alkine. The structure and the bonding arrangement of the compound were determined by single crystal X-ray diffraction studies and DFT calculation.

Chapter 6 documents the synthesis of new alkine analogue of germanium. The bis-germylene has an identical geometry as that of bis-silylene. Furthermore we exhibited its reactivity towards azobenzene and diiron nonacarbonyl. In the first case there was a cleavage of the GeGe bond whereas in the latter case the Ge-Ge bond remained intact.

Chapter 7 accounts the synthesis, and structures of amidinato stabilized divalent tin(II) chloride and the metathesis reaction with $\mathrm{AgSO}_{3} \mathrm{CF}_{3}$ and (TMS) $)_{2} \mathrm{NLi}$ afforded the formation of $\mathrm{PhC}(\mathrm{N} t \mathrm{Bu})_{2} \mathrm{SnOTf}$ and $\mathrm{PhC}(\mathrm{N} t \mathrm{Bu})_{2} \mathrm{SnN}(\mathrm{TMS})_{2}$. Furthermore it was reacted with $\mathrm{Fe}_{2}(\mathrm{CO})_{9}$ to afford a stannylene iron carbonyl Lewis acid-base adduct. 


\section{Chapter 2}

\section{Convenient and High Yield Access to Silylene and its}

\section{Reactivity towards Unsaturated Compounds}

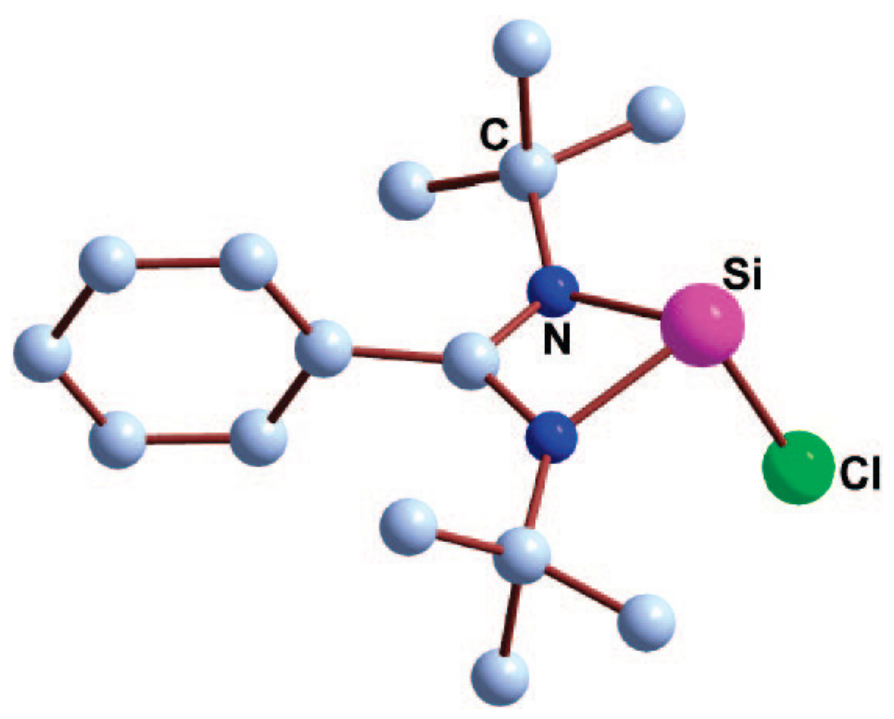

\section{Abstract}

Two new approaches for synthesizing $\mathrm{LSiCl},\left(\mathrm{L}=\mathrm{PhC}(\mathrm{N} t \mathrm{Bu})_{2}\right)$ are reported by the reaction of $\mathrm{LSiHCl}_{2}$ with bis-trimethylsilyl lithium amide and $\mathrm{N}$-heterocyclic carbene respectively. In the former method silylene is produced in $90 \%$ yield. Furthermore the silylene was treated with different unsaturated compounds e.g. biphenyl alkine, carbodiimide, adamentyl azide, tertbutylisocyanate, ketone, 1,2-diketone, diimine, phosphaalkine, COT which afforded a series of novel silicon containing heterocycles. These results are documented herein in order to facilitate future efforts in uncovering the silylene chemistry. 


\subsection{Introduction}

It is a profound and necessary truth that the deep things in science are not found because they are useful; they are found because it was possible to find them.

Robert Oppenheimer

Although more than two decades ago no one thought that silylene could be stable at room temperature, today silylene is even "bottleable". Numerous properties and characteristics of silylene have been measured and calculated. The realm of silylene chemistry has been expanded dramatically, and the investigation of reactivity of $\mathrm{N}$-heterocyclic silylene has become a matter of great interest. As mentioned before there is no example of a stable monochlorosilylene of the type $\mathrm{LSiCl}\left(\mathrm{L}=\mathrm{PhC}(\mathrm{N} t \mathrm{Bu})_{2}\right)$ known that contains a $\mathrm{Si}(\mathrm{II})-\mathrm{Cl}$ bond. We were able to achieve this milestone by exploiting the donor ability of the benz-amidinato ligand $^{25 \mathrm{a}}$ with the tert-butyl substituents on the nitrogen atoms. The 1:1 reaction of tertbutylcarbodiimide with phenyllithium in diethyl ether gave the amidinatolithium compound 20, which upon subsequent treatment with silicon tetrachloride afforded the amidinatotrichlorosilane 21a in $47 \%$ yield. The reduction with 2 equivalents of finely divided potassium at room temperature resulted in amidinatochlorosilylene $\mathbf{1 8}$ as colorless crystals (10\% yield) (Scheme 2.1). In order to prepare chlorosilylenes with different substituents on the ligand backbone, reduction of amidinatotrichlorosilane (containing trimethylsilyl groups on nitrogen atoms) was carried out with 2 equivalents of potassium. Instead of the expected chlorosilylene $\left[\mathrm{PhC}(\mathrm{NTMS})_{2}\right] \mathrm{SiCl}$, a compound with hexa-coordinate silicon atom was formed.

By adopting the protocol used for the synthesis of chlorosilylene 18, we aimed at the synthesis of unusual heteroleptic silylene $\mathrm{LSiR}\left(\mathrm{L}=\mathrm{PhC}(\mathrm{N} t \mathrm{Bu})_{2}\right)\left(\mathrm{R}=\mathrm{NMe}_{2}, \mathrm{PiPr}_{2}, \mathrm{O} i\right.$ $\mathrm{Pr}, \mathrm{O} t \mathrm{Bu})$. Accordingly, substituted amidinatodichlorides $\left[\mathrm{PhC}(\mathrm{N} t \mathrm{Bu})_{2}\right] \mathrm{SiCl}_{2} \mathrm{R} ; \mathrm{R}=\mathrm{NMe}_{2}$ 21b, $\mathrm{O} i \mathrm{Pr} 21 \mathbf{c}, \mathrm{O} t \mathrm{Bu}$ 21d, $\mathrm{P} \mathrm{Pr}_{2}$ 21e) were prepared by a $1: 1$ reaction of 21 a with $\mathrm{RLi}(\mathrm{R}=$ 
$\left.\mathrm{NMe}_{2}, \mathrm{P} i \mathrm{Pr}_{2}, \mathrm{O} i \mathrm{Pr}, \mathrm{O} t \mathrm{Bu}\right)$ and reduced with 2 equivalents of potassium at ambient temperature (Scheme 2.1). ${ }^{37}$

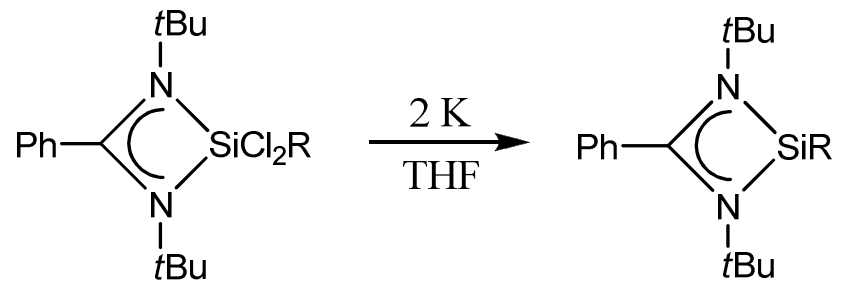

$\mathrm{R}=\mathrm{Cl}$ 21a, $\mathrm{NMe}_{2}$ 21b, O $i \mathrm{Pr}$ 21c, $\mathrm{O} t \mathrm{Bu}$ 21d, $\mathrm{P} \mathrm{Pr}_{2} 21 \mathbf{e}$

Scheme 2.1. Synthesis different heteroleptic silylenes

After being successful in preparing a number of heteroleptic silylenes we were curious to study its reactivity because little is known about the chemistry of substituted silylenes. The detailed DFT calculation predicted increased reactivity due to the presence of the stereochemically active lone pair at the $\mathrm{Si}$ atom, but the low yield of $\mathrm{LSiCl}$ hindered our further investigation. This situation may be largely related with the very limited synthetic approaches. The earlier routes to transient silylenes commonly involved photolytic and high temperature elimination of small organic fragments from appropriate silanes. ${ }^{26}$ But the most common route is the reduction of the parent halogen compounds with strong reducing agents such as potassium metal or potassium graphite $\left(\mathrm{KC}_{8}\right)$. Therefore, all the reported synthetic routes demand either rigorous conditions or highly reactive metallic reagents. Hence, there is a high quest to explore an alternative route to silylenes under mild conditions.

Very recently we showed that N-heterocyclic carbene could behave as a dehydrochlorinating agent and were able to isolate Lewis base stabilized dichloro silylene and activated germylene. ${ }^{25 b, 38}$ Following this, Cui et al. reported the isolation of four- and five- membered silylenes with the same approach. ${ }^{39}$ Benkeser and his coworkers discussed the formation of $\mathrm{SiCl}_{3}$ anion when trichlorosilanes are treated with amines. ${ }^{40 \mathrm{a}}$ Karsch and coworkers showed that the reaction of trichlorosilanes with tertiary amine provides the $\mathrm{SiCl}_{3}$ anion. ${ }^{40 \mathrm{~b}}$ Recently 
Driess et al. demonstrated that $\operatorname{LiN}(\mathrm{TMS})_{2}$ can be used as a dehydrochlorinating agent to prepare the activated germylene. ${ }^{41}$ Consequently we employed these two techniques and found out two new routes for the preparation of $\mathrm{LSiCl}$ in good yields.

\subsection{New Syntheses of $\mathrm{PhC}(\mathrm{N} t \mathrm{Bu})_{2} \mathrm{SiCl}(18)$}

In the new procedure we reacted $\mathrm{HSiCl}_{3}$ with tert-butyl carbodiimide and phenyl lithium to yield $\mathrm{LSiHCl}_{2}$ (21). The compound was characterized by NMR spectroscopy, EI-MS spectrometry, and elemental analysis. In the ${ }^{29} \mathrm{Si}$ NMR a sharp resonance was observed at $96.8 \mathrm{ppm}$. In the EI-MS spectrum the molecular ion is observed as the most abundant peak at $m / z 331$ with highest relative intensity. All these data confirm the proposed chemical constitution of 21 .

A toluene solution of $\mathrm{LSiHCl}_{2}$ and 1,3-di-tert-butylimidazol-2-ylidene under stirring immediately changed the color from colorless to yellow and finally to brown-red with the formation of a white precipitate. The insoluble white precipitate was identified as 1,3-di-tertbutylimidazolium chloride and the soluble part as the silylene $\mathbf{1 8}$ with $35 \%$ yield, which was confirmed by NMR spectroscopy, EI-MS spectrometry, and compared to a previously reported sample (Scheme 2.2). However 18 is easily accessible in $90 \%$ yield from the direct reaction of 21 with $\mathrm{LiN}(\mathrm{TMS})_{2}$ as a base in molar ratio of 1:1 in toluene. In this case also the color gradually changes from colorless to yellow to finally reddish-brown. (TMS $)_{2} \mathrm{NH}$ was removed under vacuum and $\mathrm{LiCl}$ was eliminated through filtration. The advantage of $\mathrm{LiN}(\mathrm{TMS})_{2}$ over carbene is its commercial availability. The increase in the yield for $\mathbf{1 8}$ with the new method allows investigating its reactivity because the reactivity studies only spill the bean about the structure and bonding characteristics of this unique species. 


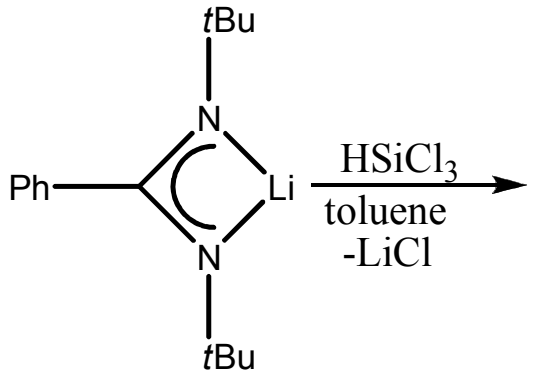

20<smiles></smiles>

21

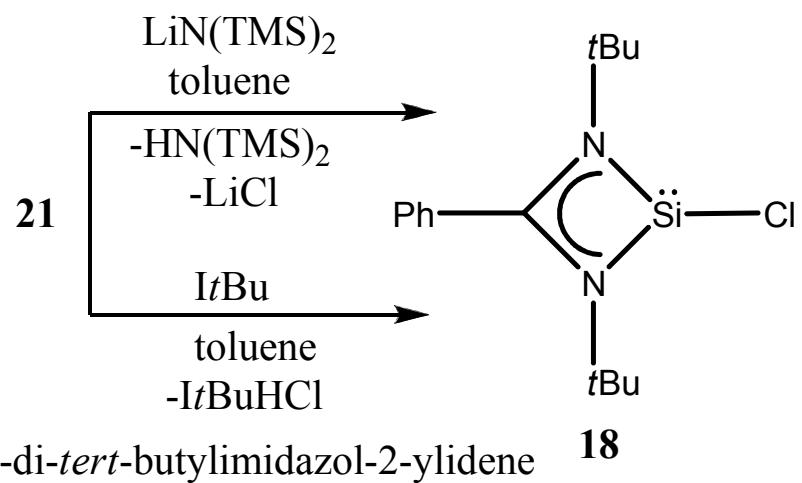

Scheme 2.2. Preparation of 21 and 18

\subsection{Reactivity of $\mathrm{LSiCl}\left(\mathrm{L}=\mathrm{PhC}(\mathrm{N} t \mathrm{Bu})_{2}\right)$ towards unsaturated} organic compounds: Synthesis of novel silicon containing ring

\section{compounds}

Silicon-containing small ring compounds are of interest because of their versatile role as building blocks in organosilicon chemistry. ${ }^{42}$ In order to derivatize 18 reactions were performed with biphenyl alkine, 2,6-diisopropyl carbodiimide, benzophenone, benzil, tertbutyl isocyante, methyl- $N$-oxide, COT, biphenylbisulfide, adamantyl azide, and phosphaalkine. In this part of the thesis we will discuss the synthesis and characterization of this novel silicon containing heterocycles in succession prepared by the reaction of $\mathbf{1 8}$ with the above-named unsaturated organic compounds. To analyse the nature of the Si center in 
these compounds we utilized ${ }^{29} \mathrm{Si}$ NMR as a probe. Furthermore the molecular structures of all these compounds were confirmed by single crystal X-ray studies.

\subsubsection{Reaction of $\mathrm{LSiCl}$ with biphenyl alkine}

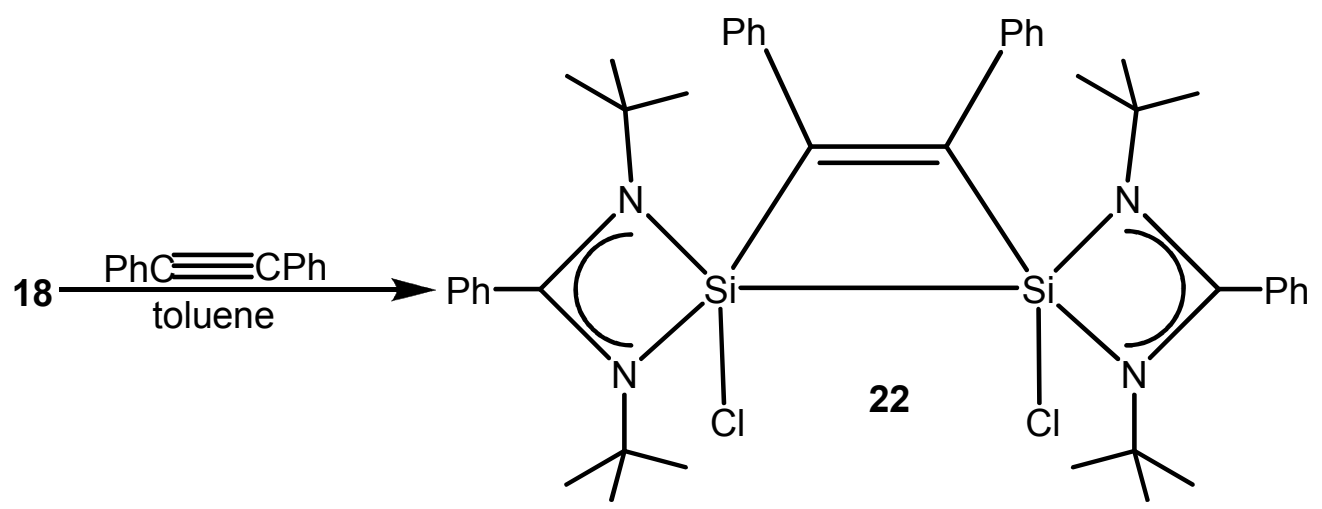

Scheme 2.3.1. Preparation of 22

$\mathrm{LSiCl}$ was reacted with biphenyl alkine in toluene at ambient temperature under stirring overnight (Scheme 2.3.1). The solution was concentrated and kept for crystallization. After 4 days colorless crystals of 1,2-disilacyclobutene $\mathbf{2 2}$ were obtained suitable for X-ray crystallography. The ${ }^{29} \mathrm{Si}$ NMR shows a resonance at $-109.53 \mathrm{ppm}$. The value is consistent with that of the reported trisilacyclopentane derivative ${ }^{25 b}$ and also with those known for cyclic silicon compounds. ${ }^{43}$

The molecular structure of $\mathbf{2 2}$ is shown in Fig. 2.3.1. ${ }^{44}$ Compound $\mathbf{2 2}$ crystallizes in the monoclinic space group $P 2{ }_{1} / c$. The $\mathrm{Si}$-Si bond length of $2.36(4) \AA$ is comparable with those of compounds containing Si-Si single bonds. ${ }^{45}$ The distance between the two carbon atoms in the ring (1.36(12) $\AA$ ) corresponds to a carbon carbon double bond. The Si-C bond distances are (1.92(9) $\AA$ and 1.93(9) $\AA$ ) in the expected range. ${ }^{46}$ The most important feature is the fourmembered $\mathrm{Si}_{2} \mathrm{C}_{2}$ ring that is almost planar (sum of the internal angles $357.82^{\circ}$ ). The amidinate ligands and chlorine atoms are disposed above and below the $\mathrm{Si}_{2} \mathrm{C}_{2}$ ring in such a way that the 
Si centers exhibit pyramidal coordination sites. The two five-coordinate silicon centers arranged adjacent to each other in a four- membered ring have not been reported so far.

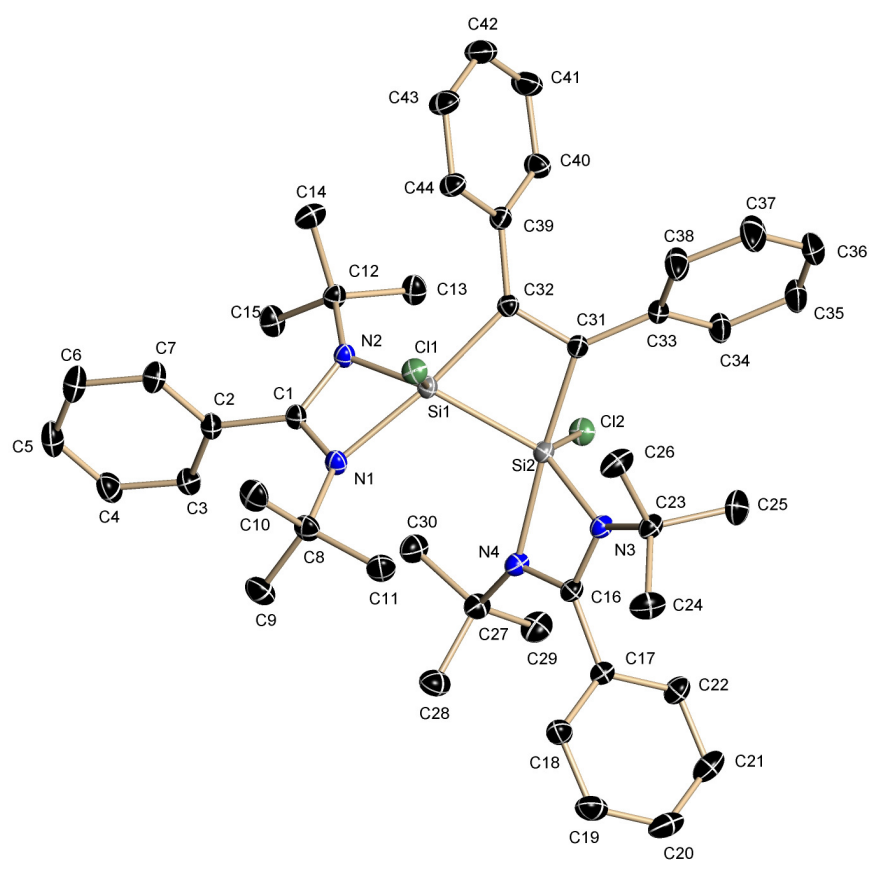

Figure 2.3.1. Crystal Structure of 22. Hydrogen atoms are not shown for clarity. Selected bond distances (Å) and bond angles (deg) Si(1)-N(2) 1.83(8), Si(1)-C(32) 1.92(9), Si(1)-N(1) 1.99(8), $\mathrm{Si}(1)-\mathrm{Cl}(1)$ 2.16(3), Si(1)-Si(2) 2.36(4), Si(2)-N(3) 1.82(8), Si(2)-C(31) 1.93(9), Si(2)$\mathrm{N}(4)$ 2.03(9), Si(2)-Cl(2) 2.15(3), C(31)-C(32) 1.36(12); N(2)-Si(1)-C(32) 108.30(4), N(2)$\mathrm{Si}(1)-\mathrm{N}(1)$ 68.46(3), C(32)-Si(1)-N(1) 173.99(4), N(2)-Si(1)-Cl(1) 117.83(3), C(32)-Si(1)-Cl(1) 90.40(3), N(1)-Si(1)-Cl(1) 86.88(3), N(2)-Si(1)-Si(2) 124.71(3), C(32)-Si(1)-Si(2) 74.76(3), $\mathrm{N}(1)-\mathrm{Si}(1)-\mathrm{Si}(2)$ 111.25(3), Cl(1)-Si(1)-Si(2) 117.322(14), N(3)-Si(2)-C(31) 109.78(4), N(3)$\mathrm{Si}(2)-\mathrm{N}(4)$ 67.91(3), C(31)-Si(2)-N(4) 175.62(4), N(3)-Si(2)-Cl(2) 114.46(3), C(31)-Si(2)-Cl(2) 91.59(3), N(4)-Si(2)-Cl(2) 86.18(3), N(3)-Si(2)-Si(1) 126.47(3), C(31)-Si(2)-Si(1) 74.54(3), $\mathrm{N}(4)-\mathrm{Si}(2)-\mathrm{Si}(1)$ 109.83(3), Cl(2)-Si(2)-Si(1) 118.779(14).

To give a mechanistic insight of the reaction we postulate that initially there is an oxidative addition between chloro silylene and biphenyl alkine resulting in the formation of a strained three-membered ring. Usually such type of cycloaddition or oxidative addition reaction is very common for heavier group 14 elements with unsaturated hydrocarbon. ${ }^{47}$ The strained three-membered ring undergoes a facile rearrangement by the insertion of another silylene molecule, thus giving rise to a stable four-membered disilacyclobutene system with formation of a Si-Si bond (Scheme 2.3.2). This is an oxidative addition followed by insertion reaction which is novel in the case of a $\mathrm{Si}(\mathrm{II})$ system. 


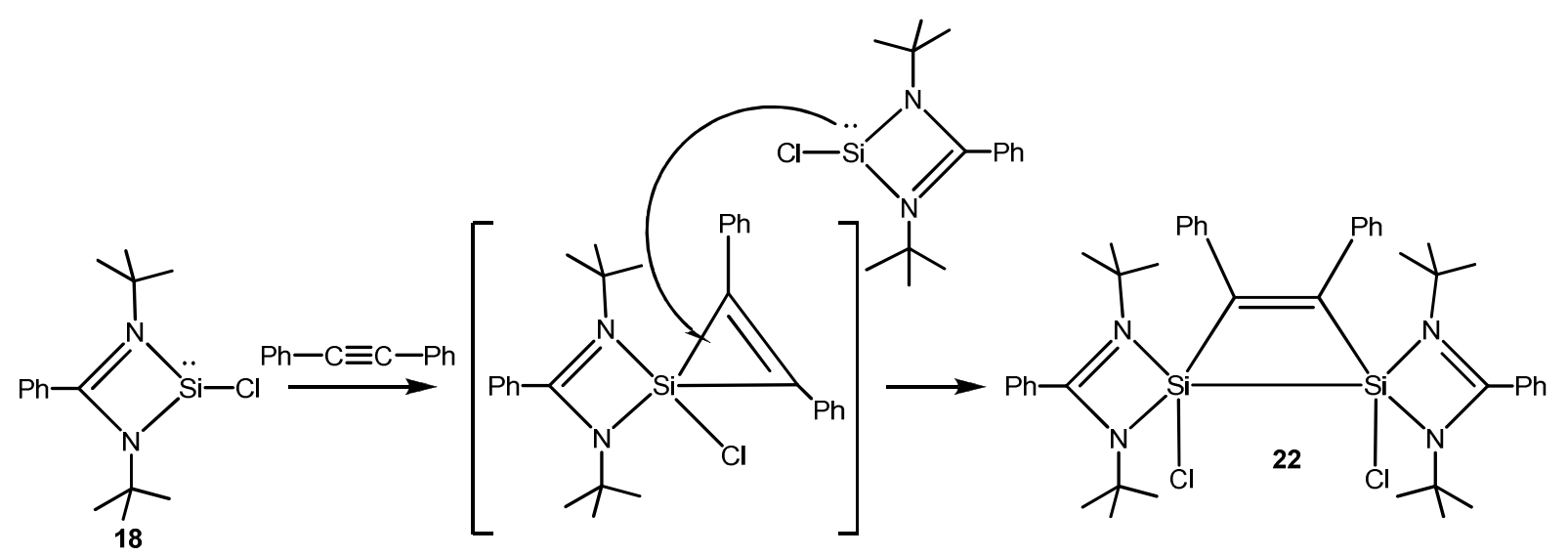

Scheme 2.3.2. Suggested mechanism for the formation of 22

Compound $\mathbf{2 2}$ is easily converted to the corresponding fluorine derivative $\mathbf{2 3}$ using trimethyltin fluoride as a fluorinating agent (Scheme 2.3.3). Compound $\mathbf{2 3}$ is a colorless solid and soluble in solvents like toluene, ether, and THF. 23 was characterized by ${ }^{1} \mathrm{H},{ }^{19} \mathrm{~F}$, and ${ }^{29} \mathrm{Si}$ NMR spectroscopy. In the ${ }^{19} \mathrm{~F}$ NMR spectrum 23 appeared as a sharp singlet at $-71.73 \mathrm{ppm}$ with silicon satellite $\left(J_{\mathrm{Si}-\mathrm{F}}=254.22 \mathrm{~Hz}\right)$. The values are consistent with those reported in literature.

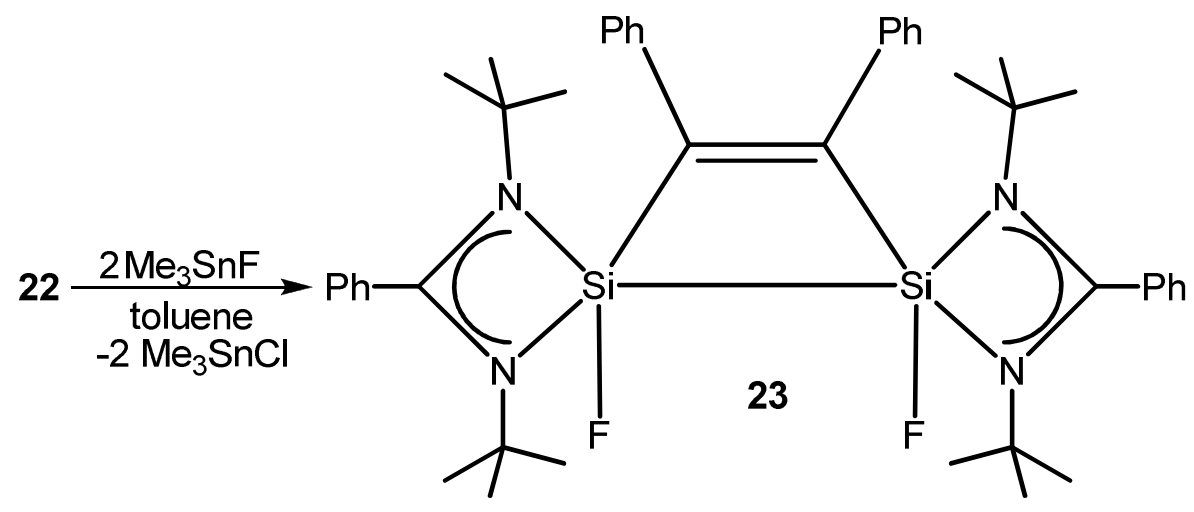

Scheme 2.3.3. Synthesis of 23 


\subsubsection{Reaction of $\mathrm{LSiCl}$ with ketone and diketone}<smiles>CC(C)(C)N1C(c2ccccc2)N(C(C)(C)C)[Si]12OC2(c1ccccc1)c1ccccc1</smiles>

24<smiles>CC(C)(C)N1C(c2ccccc2)N(C(C)(C)C)[Si]12OC(c1ccccc1)=C(c1ccccc1)O2</smiles>

25

Scheme 2.3.4. Reaction of chloro silylene with ketone and diketone

18 was reacted with benzophenone in toluene at room temperature, which afforded monosilicon epoxide (24) (Scheme 2.3.4). Similar kind of monosilicon epoxide was obtained when 19 was reacted with benzophenone in a similar manner. Furthermore 18 was reacted with benzil to yield monosiladioxolane derivative (25) (Scheme 2.3.4). The formation of 24 and 25 as stable compounds indicates that silaoxiranes can be electronically stabilized by $\sigma$ donor ligands. Compounds $\mathbf{2 4}$ and $\mathbf{2 5}$ are colorless crystals, stable under inert atmosphere and soluble in common organic solvents. The molecular structures were established by single crystal X-ray diffraction studies. The ${ }^{1} \mathrm{H}$ NMR spectra of $\mathbf{2 4}$ and $\mathbf{2 5}$ exhibit two resonances for amidinate moiety. The ${ }^{29} \mathrm{Si}$ NMR spectra of $\mathbf{2 4}$ and $\mathbf{2 5}$ exhibit sharp resonances $(\delta-$ 115.53, and $-92.24 \mathrm{ppm}$ ) consistent with fivefold-coordinate silicon. ${ }^{43}$ In the EI-MS spectra the molecular ion is observed as the most abundant peak with highest relative intensity at $\mathrm{m} / \mathrm{z}$ 476 and 505 for $\mathbf{2 4}$ and $\mathbf{2 5}$, respectively.

The molecular structure of $\mathbf{2 4}$ is shown in Figure 2.3.2. 24 crystallizes in the monoclinic space group $P 2{ }_{1} / c{ }^{44}$ In the spirocyclic structure the Si atom is part of a four- and a three- membered ring. The coordination environment of the $\mathrm{Si}$ atom exhibits a distorted square-pyramidal geometry. The coordination sites of the $\mathrm{Si}$ atom are occupied by the $\mathrm{N}$ atoms of the amidinato 
ligand, one oxygen and one carbon atom from the epoxide ring. The fifth coordination site is occupied by a chlorine atom. The $\mathrm{Si}-\mathrm{O}$ and $\mathrm{Si}-\mathrm{C}$ bond distances are $1.6435 \AA$ and $1.8641 \AA$, respectively. The $\mathrm{Si}-\mathrm{Cl}$ distance in $\mathbf{2 4}$ is $2.0708(6) \AA$, which is shorter compared to that in $\mathbf{1 8}$ (2.156(1) $\AA$ ). All these bond lengths are comparable with those of monosila epoxide formed between the reaction of $\mathbf{1 9}$ and benzophenone.

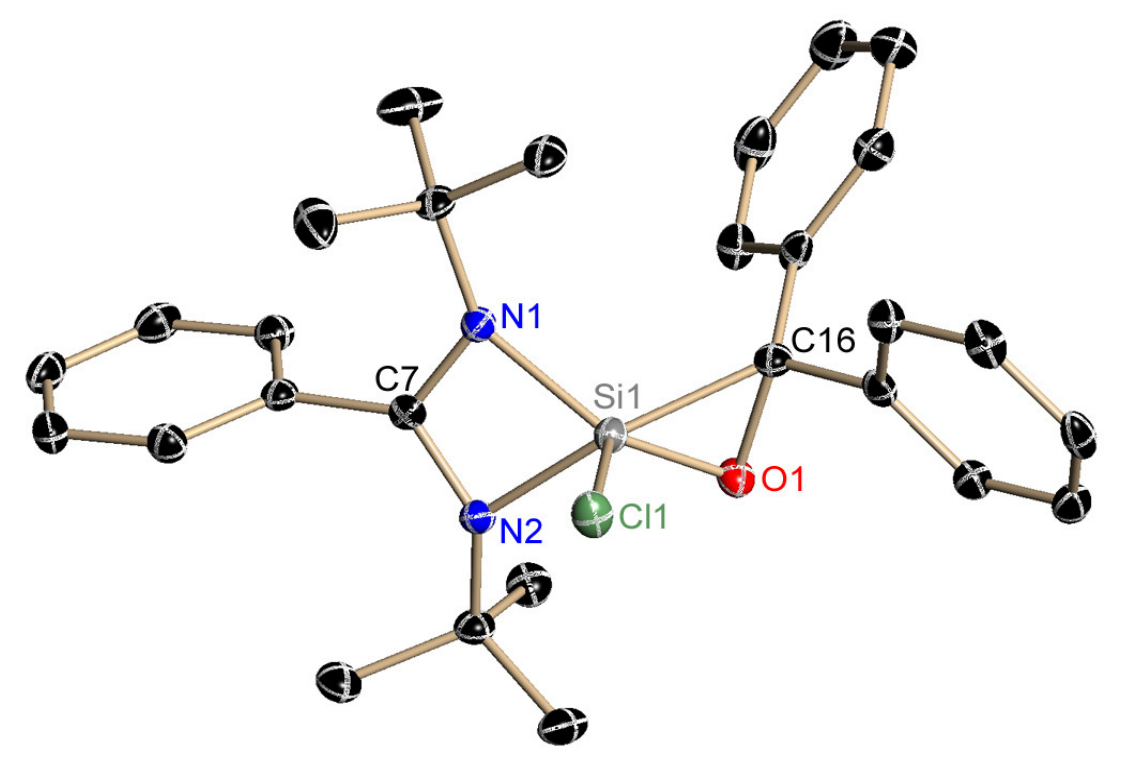

Figure 2.3.2. Molecular structure of 24; anisotropic displacement parameters depicted at the $50 \%$ probability level. Two toluene molecules were omitted for clarity. Selected bond lengths $[\AA]$ and bond angles (degree): $\mathrm{Si}(1)-\mathrm{O}(1)$ 1.6435(13), $\mathrm{Si}(1)-\mathrm{C}(16)$ 1.8641(19), $\mathrm{Si}(1)-\mathrm{Cl}(1)$ 2.0708(6), C(16)-O(1)-Si(1) 71.70(9), O(1)-Si(1)-C(1) 94.54(5), C(28)-Si(1)-C(1) 143.06(6), $\mathrm{O}(1)-\mathrm{Si}(1)-\mathrm{N}(1)$ 133.01(7), O(1)-Si(1)-N(2) 100.28(6), O(1)-Si(1)-C(16) 50.93(7), O(1)-Si(1)$\mathrm{Cl}(1) 118.15(5)$.

The structure of $\mathbf{2 5}$ was unequivocally elucidated by single crystal X-ray diffraction. The compound crystallises in the monoclinic space group $P 2_{1} / c$. The important bond lengths and bond angles are given in the legend of Figure 2.3.3. The X-ray structure revealed that the silicon center was penta-coordinate and exhibits a distorted square planar geometry. The two nitrogen atoms from amidinato ligand and two oxygen atoms from the diketone form the base of the pyramid and occupy the four coordination sites. The remaining coordination site is occupied by chlorine atom. The silicon oxygen bond lengths are 1.6796 (13) $\AA$, and 1.7064 (13) $\AA$ respectively which match very well with the reported silicon oxygen single bond 
distance. The $\mathrm{Si}-\mathrm{Cl}$ distance in 25 is (2.0958 (7) $\AA$ ), which is shorter compared to that in $\mathbf{1 8}$ (2.156(1) ^) and comparable with 24 .

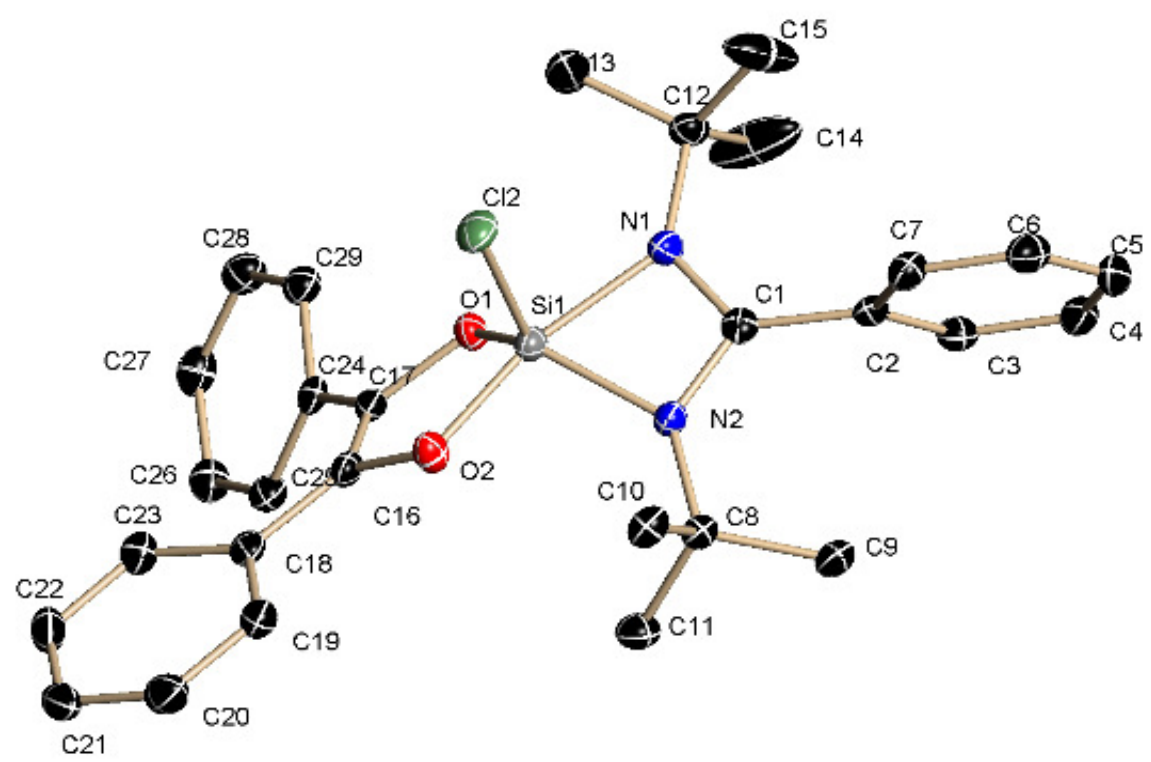

Figure 2.3.3. Crystal structure of 25. Hydrogen atoms are not shown for clarity. Anisotropic displacement parameters are depicted at the $50 \%$ probability level. Selected bond distances $(\AA)$ and bond angles (deg) $\mathrm{Cl}(2)-\mathrm{Si}(1)$ 2.0958(7), N(1)-C(1) 1.314(2), N(1)-C(12) 1.492(2), $\mathrm{N}(1)-\mathrm{Si}(1)$ 1.9091(16), N(2)-C(1) 1.363(2), N(2)-C(8) 1.493(2), N(2)-Si(1) 1.8133(16), O(1)$\mathrm{C}(17)$ 1.400(2), O(1)-Si(1) 1.6796(13), O(2)-C(16) 1.379(2), O(2)-Si(1) 1.7064(13); C(1)$\mathrm{N}(1)-\mathrm{C}(12)$ 130.76(16), C(1)-N(1)-Si(1) 90.43(11), C(12)-N(1)-Si(1) 138.55(13), C(1)-N(2)$\mathrm{C}(8)$ 132.02(15), $\mathrm{C}(1)-\mathrm{N}(2)-\mathrm{Si}(1)$ 93.08(11), C(8)-N(2)-Si(1) 134.39(12), C(17)-O(1)-Si(1) 112.30(11), $\quad \mathrm{C}(16)-\mathrm{O}(2)-\mathrm{Si}(1) \quad 111.95(11), \quad \mathrm{O}(1)-\mathrm{Si}(1)-\mathrm{O}(2) \quad 90.71(6), \quad \mathrm{O}(1)-\mathrm{Si}(1)-\mathrm{N}(2)$ 125.79(7), O(2)-Si(1)-N(2) 99.02(7), O(1)-Si(1)-N(1) 92.29(7), O(2)-Si(1)-N(1) 168.23(7), $\mathrm{N}(2)-\mathrm{Si}(1)-\mathrm{N}(1)$ 70.09(7), O(1)-Si(1)-Cl(2) 120.80(5), O(2)-Si(1)-Cl(2) 95.15(5).

\subsubsection{Reaction of $\mathrm{LSiCl}$ with carbodiimide}

Carbodiimide is a functional group consisting of the general formula $\mathrm{RN}=\mathrm{C}=\mathrm{NR}$ and $\mathrm{a}$ representative of the family of heterocumulenes. The reaction of NHC with diisopropylcarbodiimide, giving rise to the corresponding betaines followed by cyclization has been described recently. ${ }^{48}$ However, we are not aware of comparable reactions with silylenes. 18 was reacted with 2,6-diisopropyl phenyl carbodiimide in toluene at ambient temperature under stirring overnight. After that the solution was concentrated and kept for crystallisation, which affords colorless crystals of $\mathbf{2 6}$ suitable for X-ray crystallography 
(Scheme 2.3.4). The compound is soluble in toluene, diethyl ether, and THF. The structure of 26 was also confirmed by NMR spectroscopy, EI-MS spectrometry and elemental analysis. The ${ }^{1} \mathrm{H}$ NMR spectrum exhibits a resonance $\delta 1.27 \mathrm{ppm}$ which corresponds to the $t \mathrm{Bu}$ protons. Two sharp resonances at $\delta 1.20$ and $1.21 \mathrm{ppm}$ indicate the twelve $\mathrm{CH}_{3}$ protons. One septet was observed $\delta$ 3.5-3.6 ppm and another septet was obserevd $\delta$ 4.0-4.1 ppm for the two $\mathrm{CH}$ protons of the isopropyl group. The ${ }^{29} \mathrm{Si}$ NMR spectrum exhibits a sharp resonance at $\delta-104.73 \mathrm{ppm}$. In the EI-MS spectrum the molecular ion was observed as the most abundant peak at $\mathrm{m} / \mathrm{z}$ 469. All these data are in accordance with the proposed formula of $\mathbf{2 6}$. This is a very new and convenient route to prepare such silaimido complexes without using dangerous organoazides.
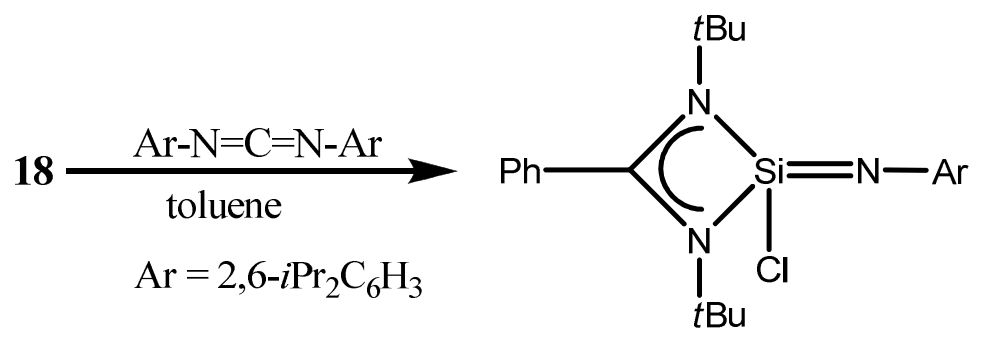

26

Scheme 2.3.4. Preparation of 26

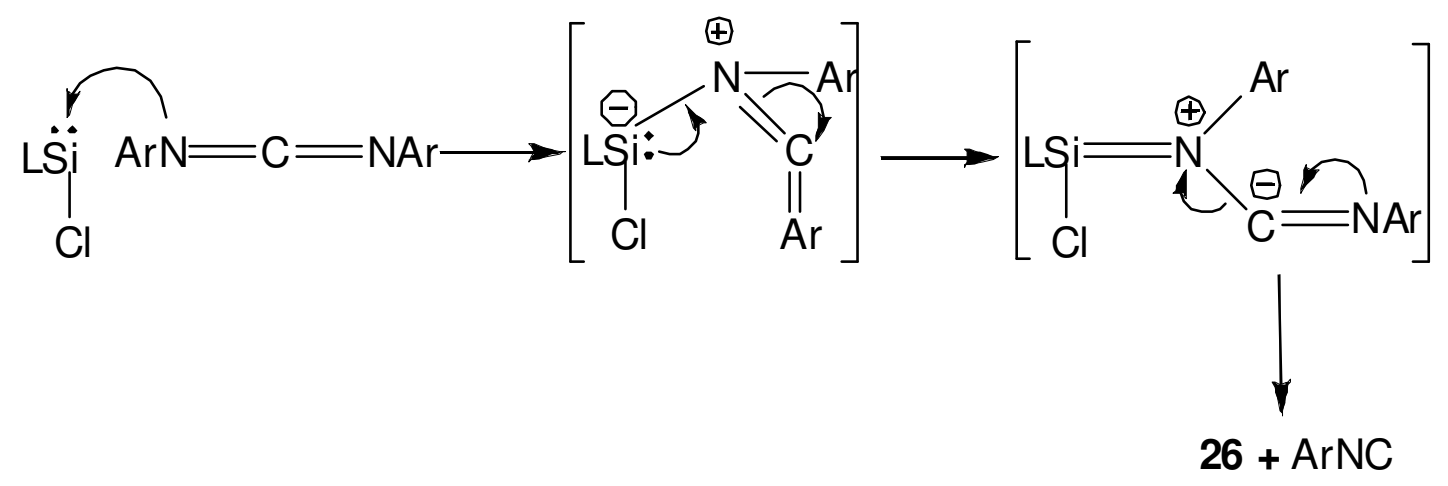

Scheme 2.3.4. Proposed mechanism of formation of 26 
We are not sure about the mechanism of formation of $\mathbf{2 6}$ and intermediates could not be isolated, but we suggest that the reaction may take place in the following way (Scheme 2.3.5). The lone pair of nitrogen attacks the electrophilic silylene first, which subsequently forms the double bond with nitrogen through the donation of its lone pair. This intermediate further rearranged to $\mathbf{2 6}$ along with the formation of isonitrile, which was proved by the ${ }^{1} \mathrm{H}$ NMR spectroscopy.

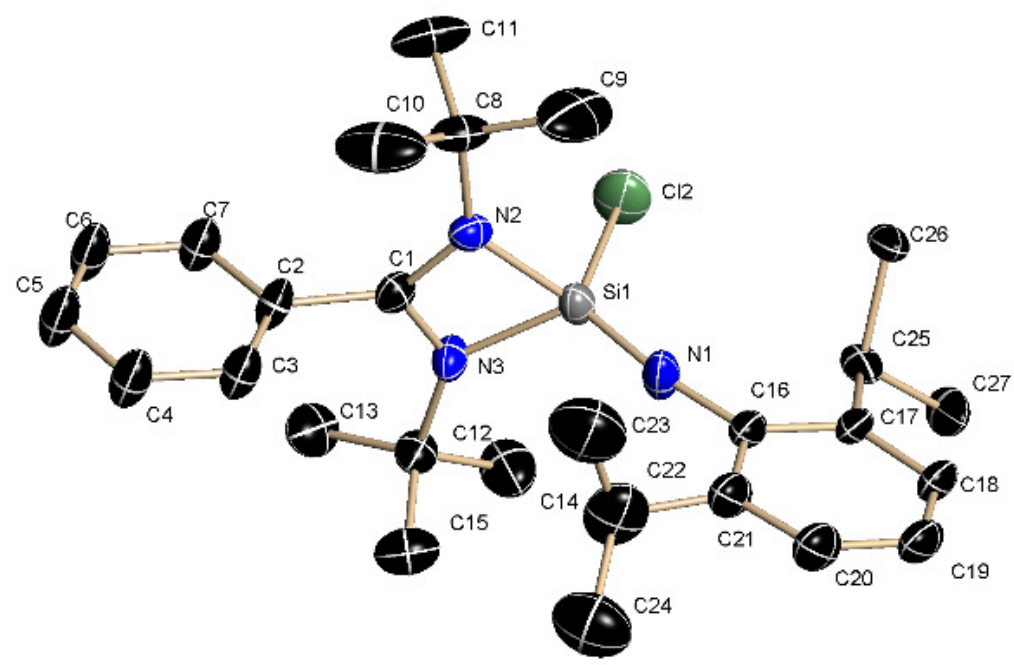

Figure 2.3.4. Crystal structure of 26. Hydrogen atoms are not shown for clarity. Anisotropic displacement parameters are depicted at the $50 \%$ probability level. Selected bond distances $(\AA ̊)$ and bond angles (deg) Si(1)-N(1) 1.545(2), Si(1)-N(3) 1.803(2), $\mathrm{Si}(1)-\mathrm{N}(2)$ 1.809(2), $\mathrm{Si}(1)-$ $\mathrm{Cl}(2) \quad 2.087(10) ; \mathrm{N}(1)-\mathrm{Si}(1)-\mathrm{N}(3) \quad 121.63(12), \quad \mathrm{N}(1)-\mathrm{Si}(1)-\mathrm{N}(2) \quad 122.71(12), \quad \mathrm{N}(3)-\mathrm{Si}(1)-\mathrm{N}(2)$ 72.73(9), N(1)-Si(1)-Cl(2) 121.33(9), N(3)-Si(1)-Cl(2) 103.57(8), N(2)-Si(1)-Cl(2) 104.10(7).

Single crystals of $\mathbf{2 6}$ were grown from a concentrated toluene solution. The molecular structure of $\mathbf{2 6}$ was determined by single crystal X-ray diffraction (Figure 2.3.4). ${ }^{44} \mathbf{2 6}$ crystallizes in the monoclinic space group $P 2_{1} / c$. The silicon center exhibits distorted tetrahedral geometry. The two sites of the silicon atom are occupied by the $\mathrm{N}$ atoms from the amidinato ligand and the other site is occupied by chlorine atom. Nitrogen atom from the carbodiimide group occupies the remaining coordination site. The most important bond lengths are the Si-N bond lengths. The two silicon and amidinato nitrogen bond lengths are almost the same (1.803(2) and 1.809(2) $\AA$ ), whereas the other $\mathrm{Si}-\mathrm{N}$ bond length is $1.545(2) \AA$ 
which clearly indicates the formation of $\mathrm{Si}=\mathrm{N}$ which is stabilized kinetically by the bulky 2,6diisopropyl phenyl group. The $\mathrm{Si}-\mathrm{Cl}$ bond length is $2.09(10) \AA$ ), which is shorter compared to that in $18(2.156(1) \AA)$.

\subsubsection{Reaction of LSiCl with adamantyl azide}

The formation of silaimine complex from the reaction between carbodiimide and $\mathbf{1 8}$ prompted us to probe the reaction of $\mathbf{1 8}$ with adamantyl azide. Recently Weidenbruch et al. reported the formation of 1,3-diaza-2-silacyclobutene ${ }^{49}$ from the reaction of adamantyl azide and silylene. Treatment of $\mathrm{AdN}_{3}$ with $\mathbf{1 8}$ in toluene solution furnished a colorless solid under elimination of nitrogen (Scheme 2.3.6). The EI-MS spectrum showed the molecular ion as the most abundant peak with highest relative intensity at $m / z, 443$ which suggested the formation of the silaimine complex 27. The NMR data are also in consistent with the proposed one. In the ${ }^{29} \mathrm{Si}$ NMR spectrum a sharp resonance was observed at $\delta-102.56 \mathrm{ppm}$. The value is in good accord with that of $\mathbf{2 6 .}$

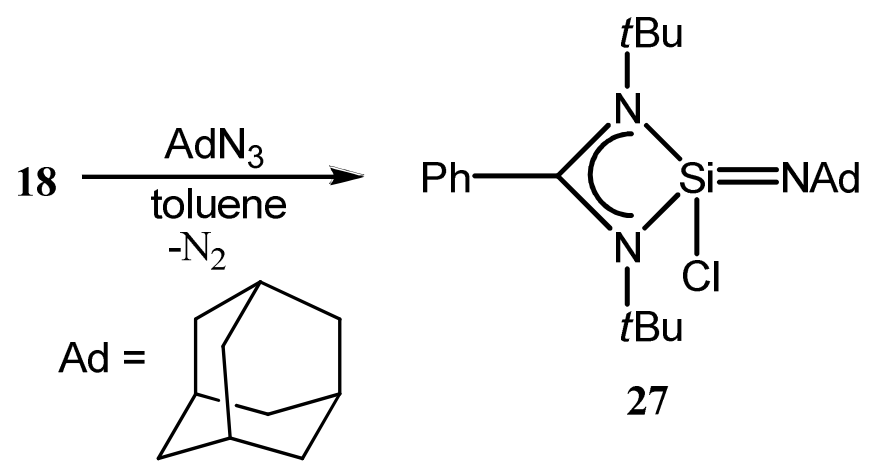

Scheme 2.3.6 Preparation of 27

\subsubsection{Reaction of $\mathrm{LSiCl}$ with 1, 3, 5, 7-octatetraene}

Cyclooctatetraene (COT), the first $4 \pi$ p-electron system to be studied, adopts an inherently nonplanar tub-shaped geometry of $D_{2 d}$ symmetry with alternating single and double bonds (with angles $\mathrm{C}=\mathrm{C}-\mathrm{C} 126.1^{\circ}$ and $\mathrm{C}=\mathrm{C}-\mathrm{H} \quad 117.6^{\circ}$ ) and hence behaves as a nonaromatic polyene rather than an anti-aromatic compound. ${ }^{50}$ COT has attracted a lot of attention because of its 
nature to change conformation between tub- and planar-shaped structure with addition or subtraction of electrons. Many COT-bridged molecules are known for $s$-block and $f$-block elements. ${ }^{51}$ Cyclic $[2+1]$ derivatives of COT with phosphorus fragments ${ }^{52}$ are known but there is to best of our knowledge no report for the reaction of COT with silylene. We have chosen COT to react with silylene to investigate the reactivity of silylene with cyclic polyolefinic system. There are few examples reported on the direct concerted cycloaddition of COT with unsaturated compounds and as a rare example, silylene $\mathbf{1 8}$ exhibits a [1+4]cycloaddition to the COT ring system.
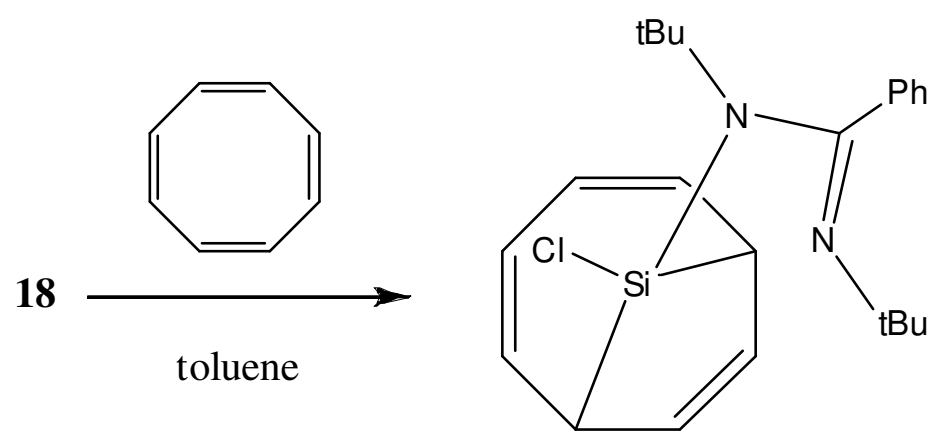

28

Scheme 2.3.7. Preparation of 28

Addition of COT to the colorless solution of $\mathbf{1 8}$ in a molar ratio of 1:1 in toluene at ambient temperature resulted in a light yellow crystalline solid after stirring overnight and removal of solvent under vacuum (Scheme 2.3.7). The solid was extracted with toluene. The colorless crystals of $\mathbf{2 8}$ were obtained by storing the saturated solution of $\mathbf{2 8}$ at room temperature for one day. The composition and constitution of $\mathbf{2 8}$ was proven by spectroscopic methods and elemental analysis. The ${ }^{1} \mathrm{H}$ NMR shows a broad resonance for the tert-butyl protons $(\delta 1.12$ $1.34 \mathrm{ppm}$ ) because two chemically different tert-butyl protons are present. The resonances at $\delta$ 2.28 and 5.63-5.93 ppm correspond to two $\mathrm{CH}$ and six $\mathrm{CH}=\mathrm{C}$ protons respectively. In the 
${ }^{29} \mathrm{Si}$ NMR a sharp resonance exhibits at $-9.46 \mathrm{ppm}$ that is in accordance with reported value for $[1+4]$ cycloaddition product. In the EI-MS spectrum the molecular ion peak with high intensity was observed at $m / z 397\left[\mathrm{M}^{+}\right]$.

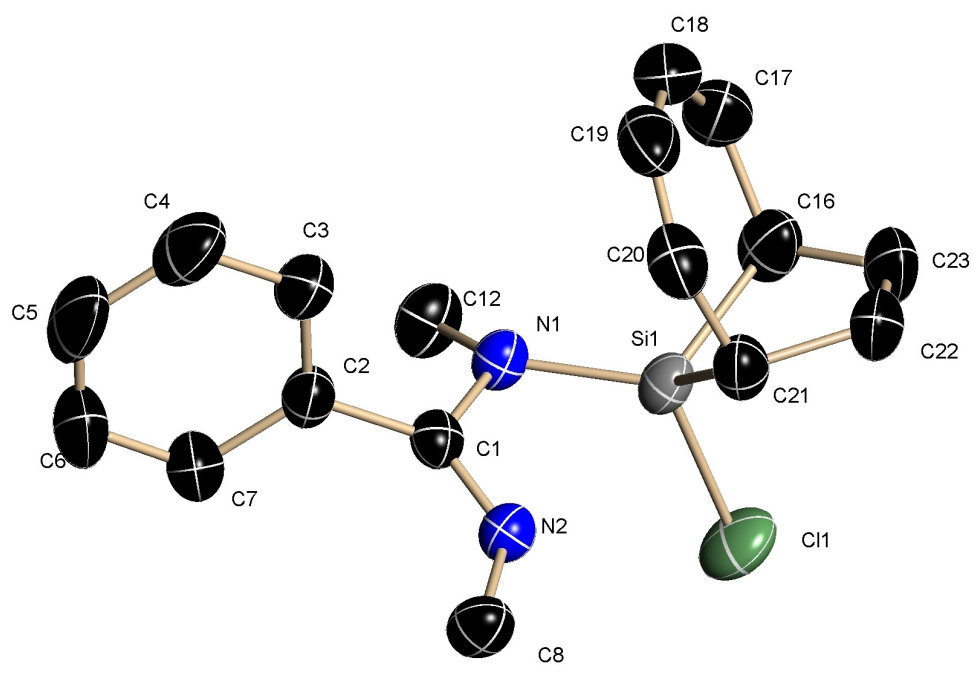

Figure 2.3.5. Crystal structure of 28. Hydrogen atoms and $t B u$ groups are not shown for clarity. Anisotropic displacement parameters are depicted at the $50 \%$ probability level. Selected bond distances $(\AA)$ and bond angles (deg) $\mathrm{Cl}(1)-\mathrm{Si}(1) 2.0820(7), \quad \mathrm{N}(1)-\mathrm{C}(1)$ 1.413(2), $\quad \mathrm{N}(2)-\mathrm{C}(1)$ 1.273(2), $\mathrm{Si}(1)-\mathrm{C}(21)$ 1.8761(19), $\quad \mathrm{Si}(1)-\mathrm{C}(16)$ 1.895(2), $\quad \mathrm{N}(1)-\mathrm{Si}(1)$ 1.7418(14), C(21)-C(22) 1.513(3), C(22)-C(23) 1.317(3), C(16)-C(23) 1.510(3); N(1)-Si(1)$\mathrm{C}(21)$ 119.34(7), $\mathrm{N}(1)-\mathrm{Si}(1)-\mathrm{C}(16)$ 114.71(8), N(1)-Si(1)-Cl(1) 114.20(6), $\mathrm{C}(21)-\mathrm{Si}(1)-\mathrm{Cl}(1)$ 111.62(6), $\quad \mathrm{C}(16)-\mathrm{Si}(1)-\mathrm{Cl}(1) \quad 103.11(7), \quad \mathrm{C}(21)-\mathrm{Si}(1)-\mathrm{C}(16) \quad 90.43(9), \quad \mathrm{C}(20)-\mathrm{C}(21)-\mathrm{C}(22)$ 108.76(16), C(22)-C(23)-C(16) 116.88(18), C(20)-C(19)-C(18) 132.0(2).

The molecular structure of $\mathbf{2 8}$ was unequivocally confirmed by single crystal X-ray diffraction (Figure 2.3.5). ${ }^{44} \mathbf{2 8}$ crystallizes in the triclinic space group $P$-1. Selected bond lengths and bond angles are given in the legend of Figure 2.3.5. The structure displays a bicyclooctatriene system with tetra-coordinate silicon. Sil shows a distorted tetrahedral geometry where two sites of the silicon center are occupied by two carbon C16 and C21 of the COT ring and another two sites are filled with nitrogen N1 of aminidato ligand and Cl1. The COT ring in its final structure has non-planar geometry with altering $\mathrm{C}-\mathrm{C}$ and $\mathrm{C}=\mathrm{C}$ bonds. The bond angles $\mathrm{C}(20)-\mathrm{C}(21)-\mathrm{C}(22)$ 108.76(16), C(22)-C(23)-C(16) 116.88(18), and $\mathrm{C}(20)-\mathrm{C}(19)-\mathrm{C}(18) \mathbf{1 3 2 . 0 ( 2 )}$ of the cycloadduct $\mathbf{2 8}$ are also in the range of the non-planar and 
distorted tub-shaped geometry of COT. The mechanism involves a rare example of a [1+4] cycloaddition of silylene to the COT ring for the formation of a silicon substituted bicyclosystem with three $\mathrm{C}=\mathrm{C}$ bonds (Scheme 2.3.8).
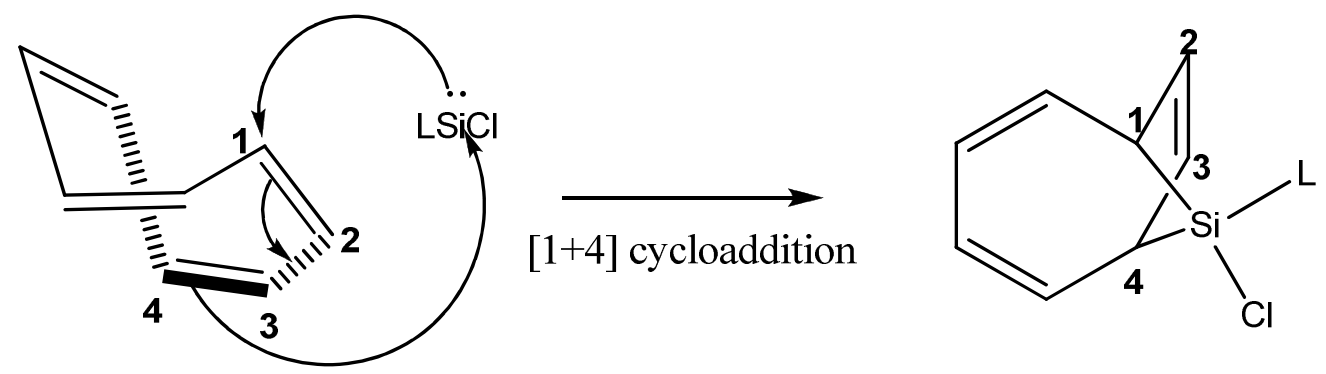

Scheme 2.3.8. Mechanism for the formation of 28

The outcome of the reaction between COT and $\mathbf{1 8}$ encouraged us to study the reaction between silylene with cyclooctadiene, and cis-cyclooctene. Unfortunately the reactions led to the formation of insoluble products which could not be identified.

\subsubsection{Reaction of $\mathrm{LSiCl}$ with tert-butylisocyanate}<smiles>CC(C)(C)N1C(c2ccccc2)N(C(C)(C)C)[Si]12O[Si]1(Cl)(Cl)(N(C(C)(C)C)C(C)(C)C)N(C(c3ccccc3)N1C(C)(C)C)C2c1ccccc1</smiles>

Scheme 2.3.9. Preparation of 29

Isocyanate displays two unsaturated functional groups and therefore may show some fascinating reactivities with stable silylenes. We are not aware that such reactions have been reported so far. We reasoned that the reaction would undergo a putative $[3+2]$ cycloaddition, but to our surprise the formation of a $\mathrm{Si}_{2} \mathrm{O}_{2}$ ring under cleavage of the $\mathrm{C}=\mathrm{O}$ bond was 
observed. The formation of tert-butyl isocyanide as a side product in the reaction was characterized by ${ }^{1} \mathrm{H}$ NMR spectroscopy.

The reaction mixture of $\mathbf{1 8}$ with tert-butylisocyanate in toluene at ambient temperature was stirred overnight and removal of solvent under vacuum afforded a colorless solid. The solid was extracted with toluene (Scheme 2.3.9). The colorless crystals of 29 were obtained by storing the concentrated toluene solution at $-32{ }^{\circ} \mathrm{C}$ in a freezer. The composition and constitution of 29 was proven by spectroscopic methods and elemental analysis. The ${ }^{1} \mathrm{H}$ NMR shows one set of resonances from the amidinato ligand. In the ${ }^{29} \mathrm{Si}$ NMR spectrum a sharp resonance exhibits at $-113.54 \mathrm{ppm}$. The value is consistent with those of the reported fivecoordinate silicon compounds. ${ }^{43}$ In the EI-MS spectrum the molecular ion peak was observed at $m / z 620$, although with small intensity.

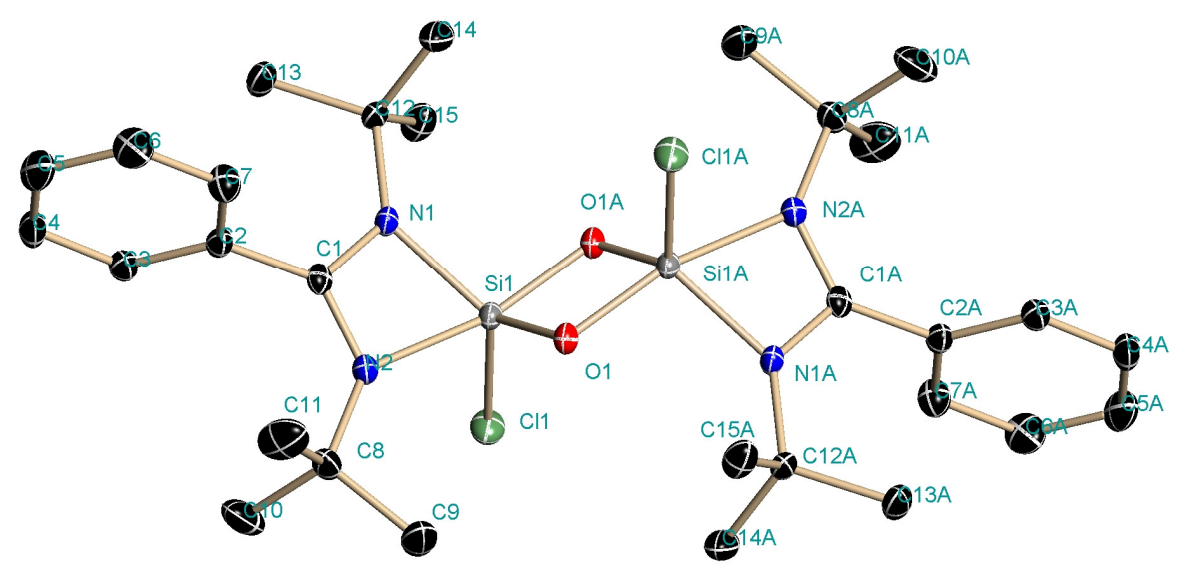

Figure 2.3.6. Crystal structure of 29. Hydrogen atoms are not shown for clarity. Anisotropic displacement parameters are depicted at the $50 \%$ probability level. Selected bond distances (Å) and bond angles (deg) O(1)-Si(1) 1.6574(12), O(1)-Si(1)\#1 1.7177(12), Si(1)-O(1)\#1 1.7177(12), $\quad \mathrm{Cl}(1)-\mathrm{Si}(1)$ 2.0972(6), $\quad \mathrm{N}(1)-\mathrm{C}(1)$ 1.356(2), $\quad \mathrm{N}(1)-\mathrm{C}(12) \quad 1.486(2), \quad \mathrm{N}(1)-\mathrm{Si}(1)$ 1.8080(14), N(2)-C(1) 1.311(2), N(2)-C(8) 1.481(2), N(2)-Si(1) 1.9416(14). O(1)-Si(1)-O(1)\#1 85.63(6), $\quad \mathrm{Si}(1)-\mathrm{O}(1)-\mathrm{Si}(1) \# 1 \quad 94.37(6), \quad \mathrm{O}(1)-\mathrm{Si}(1)-\mathrm{N}(1) \quad$ 126.02(6), $\quad \mathrm{O}(1) \# 1-\mathrm{Si}(1)-\mathrm{N}(1)$ 100.07(6), O(1)-Si(1)-N(2) 94.31(6), O(1)\#1-Si(1)-N(2) 166.96(6), N(1)-Si(1)-N(2) 69.46(6), $\mathrm{N}(1)-\mathrm{Si}(1)-\mathrm{Cl}(1)$ 113.78(5).

The molecular structure of $\mathbf{2 9}$ is shown in Figure 2.3.6. ${ }^{44}$ Compound $\mathbf{2 9}$ crystallizes in the triclinic space group $P-1$. The structure consists of a rectangular cyclodisiloxane ring 
orthogonal to a slightly distorted planar skeleton containing the silicons and their pendant nitrogens. The two independent Si-O bond lengths are very close to each other $(1.65(12)$ and 1.71(12) ̊) and are slightly larger than the normal bond lengths found for the other cyclic siloxanes. The amidinate ligands and $\mathrm{Cl}$ atoms are disposed above and below the $\mathrm{Si}_{2} \mathrm{O}_{2}$ ring in such a way that the Si centers exhibit a distorted square pyramidal geometry. The Si-Cl bond length is 2.09(6) $\AA$. Another striking feature of this structure is the Si-Si distance of 2.48(12) $\AA$ which is $0.13 \AA$ longer than the normal Si-Si sigma bond distance $(2.35 \AA)$. ${ }^{46}$ From these values it can be assumed that there is no bond between the two Si atoms. This assumption is also supported by MNDO calculations on the parent cyclodisiloxane, $\mathrm{H}_{4} \mathrm{Si}_{2} \mathrm{O}_{2}$ which also provided no evidence for bonding between silicon atoms. ${ }^{53}$ In agreement with this, recent $a b$ initio calculations on $\mathrm{H}_{4} \mathrm{Si}_{2} \mathrm{O}_{2}$ indicate that the cyclodisiloxane is best described as containing four equivalent localized $\mathrm{Si}-\mathrm{O}$ bonds with no appreciable $\sigma$ bonding between the silicon atoms.

\subsubsection{Reaction of $\mathrm{LSiCl}$ with trimethylamine $N$-oxide}

Trimethylamine $N$-oxide is well known as an oxidizing agent, which is used to convert alkyl halides to aldehydes. As $\mathbf{1 8}$ has a chlorine atom bound to silicon, we were keen to probe the reaction of $\mathbf{1 8}$ with trimethylamine $N$-oxide. It is also noteworthy that the isolation of room temperature stable silanone $\left(\mathrm{R}_{2} \mathrm{Si}=\mathrm{O}\right)$ (Kipping's dream) $)^{54,55}$ is still elusive. Keeping these possibilities in mind we treated $\mathrm{Me}_{3} \mathrm{~N}^{+} \mathrm{O}^{-}$with $\mathbf{1 8}$ in THF. The formation of $\mathbf{2 9}$ was observed which we obtained from the reaction between tert-butylisocyanate and $\mathbf{1 8}$ (Scheme 2.4.0). The NMR spectroscopic data and the EI-MS spectrometry are in accordance with those of 29.

$$
18 \underset{2 . \mathrm{THF}}{\stackrel{1 . \mathrm{Me}_{3} \mathrm{~N}^{+} \mathrm{O}^{-}}{\longrightarrow}} \mathbf{2 9}
$$

Scheme 2.4.0. Alternative synthesis of 29 


\subsubsection{Reaction of $\mathrm{LSiCl}$ with diimine}

The solution of between glyoxal-bis-(2,6-diisopropylphenyl)imine and $\mathbf{1 8}$ in toluene was stirred for 2 days. Evaporation of the solvent afforded air and moisture sensitive colorless crystals of 30. The mechanism of the product formation seems to be obvious. $\mathbf{1 8}$ underwent $[1+4]$ oxidative addition reaction with diimine to yield $\mathbf{3 0}$ (Scheme 2.4.1). Compound $\mathbf{3 0}$ was isolated as a colorless crystalline solid with good solubility in solvents such as diethyl ether, toluene, and THF. Moreover it is stable in solution or in the solid state at room temperature in an inert atmosphere. It has been characterized by spectroscopic methods, and X-ray crystallography. In the ${ }^{29} \mathrm{Si}$ NMR spectrum a sharp resonance exhibited at $\delta-101.89 \mathrm{ppm},{ }^{43}$ whereas in the EI-MS spectrum the molecular ion was observed as the most abundant peak with highest relative intensity at $m / z 670$.

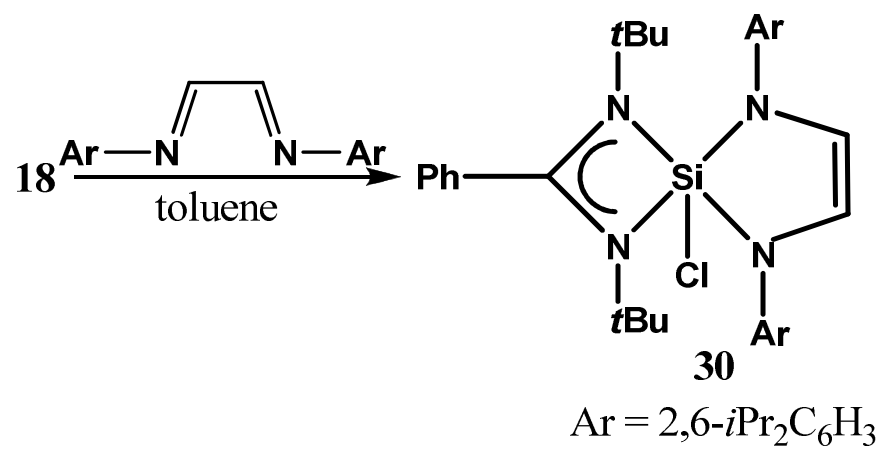

Scheme 2.4.1. Preparation of 30

Single crystals suitable for X-ray diffraction analysis were grown from saturated toluene solution of $\mathbf{3 0}$ at room temperature. The selected bond lengths and angles are given in the legend of Figure $2.3 .7 .^{44} \mathbf{3 0}$ crystallizes in the triclinic space group $P-1$. The silicon center is coordinated by two nitrogen atoms from the amidinato ligand, two nitrogen atoms from diimine, and one chlorine atom. So the silicon center displays an unambiguous distorted trigonal bipyramidal geometry with penta-coordinate silicon atom. The $\mathrm{Si}-\mathrm{N}$ bonds are the 
most notable structural features of 30. Inspection of structural data shows that $\mathrm{N}(2)$ and $\mathrm{Cl}$ occupy the axial positions with bond lengths 1.91(13) $\AA$ and 2.21(7) $\AA$, whereas the other three nitrogen atoms reside in the equatorial position. All the Si-N bond lengths are very close to each other and are in well accordance with the $\mathrm{Si}-\mathrm{N}$ single bonds reported in literature. The Si-Cl bond length in $\mathbf{8}$ is slightly longer than that in $\mathbf{1 8}$ [2.21(7) $\AA$ in $\mathbf{3 0}$ vs $2.15(1) \AA$ in $\mathbf{1 8}$ ].

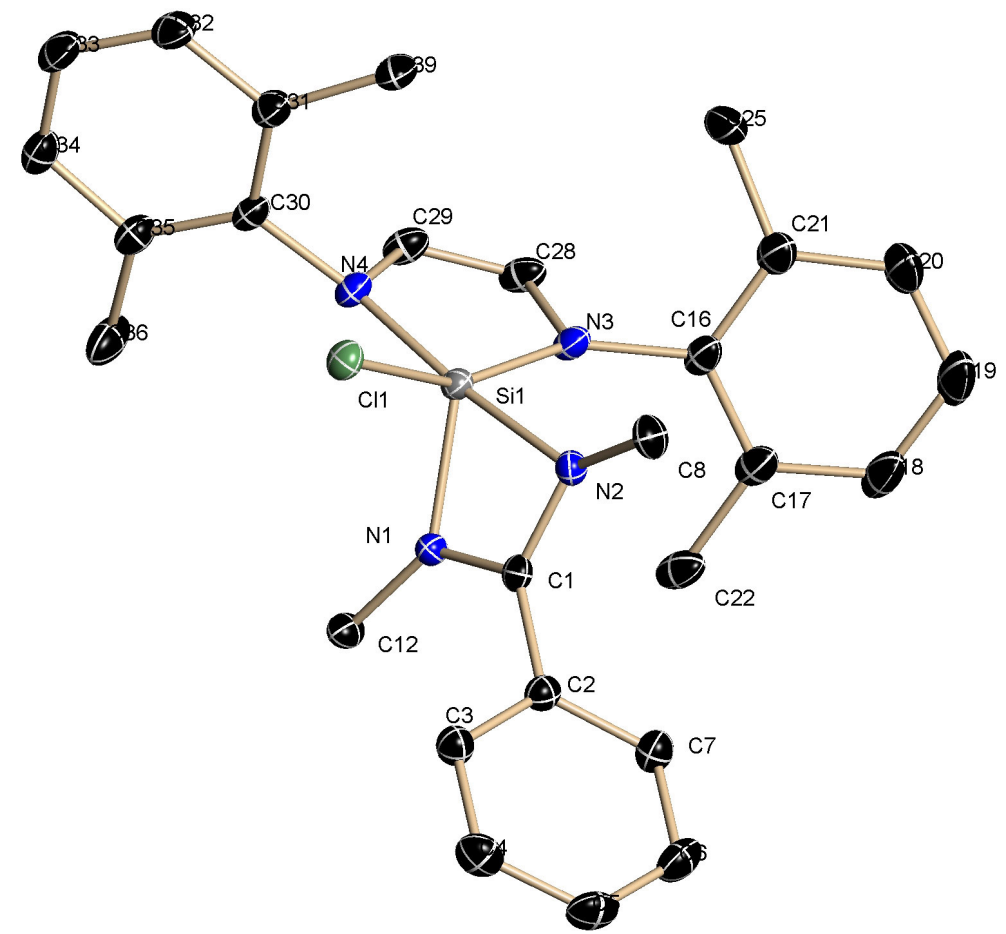

Figure 2.3.7. Crystal structure of 30 . Hydrogen atoms and $t \mathrm{Bu}$ groups are not shown for clarity. Anisotropic displacement parameters are depicted at the $50 \%$ probability level. Selected bond distances $(\AA)$ and bond angles (deg) $\mathrm{Cl}(1)-\mathrm{Si}(1) 2.2138(7), N(1)-\mathrm{C}(1)$ 1.3446(19), N(1)-C(12) 1.4862(19), N(1)-Si(1) 1.8290(13), N(2)-C(1) 1.324(2), N(2)-C(8) 1.494(2), $\quad \mathrm{N}(2)-\mathrm{Si}(1) \quad 1.9115(13), \quad \mathrm{N}(3)-\mathrm{C}(28) \quad 1.407(2), \quad \mathrm{N}(3)-\mathrm{C}(16) \quad 1.446(2), \quad \mathrm{N}(3)-\mathrm{Si}(1)$ 1.7883(14), N(4)-C(29) 1.412(2), N(4)-C(30) 1.4508(19), N(4)-Si(1) 1.7892(13), Si(1)-C(1) $2.3215(16) ; \quad \mathrm{C}(1)-\mathrm{N}(1)-\mathrm{C}(12) \quad 131.75(13), \quad \mathrm{C}(1)-\mathrm{N}(1)-\mathrm{Si}(1) \quad 92.76(9), \quad \mathrm{C}(12)-\mathrm{N}(1)-\mathrm{Si}(1)$ 135.41(10), C(1)-N(2)-C(8) 128.71(13), C(1)-N(2)-Si(1) 89.80(9), C(8)-N(2)-Si(1) 136.77(10), $\mathrm{C}(28)-\mathrm{N}(3)-\mathrm{C}(16)$ 114.44(13), $\mathrm{C}(28)-\mathrm{N}(3)-\mathrm{Si}(1)$ 111.80(10), $\mathrm{C}(16)-\mathrm{N}(3)-\mathrm{Si}(1)$ 133.42(11), C(29)-N(4)-C(30) 110.86(12), C(29)-N(4)-Si(1) 111.65(10), C(30)-N(4)-Si(1) 132.87(10), $\mathrm{N}(3)-\mathrm{Si}(1)-\mathrm{N}(4)$ 87.65(6), N(3)-Si(1)-N(1) 108.57(6), N(4)-Si(1)-N(1) 116.52(6), N(3)-Si(1)$\mathrm{N}(2)$ 92.98(6), N(4)-Si(1)-N(2) 172.86(6), N(1)-Si(1)-N(2) 70.00(6), N(3)-Si(1)-Cl(1)155.40(5), $\mathrm{N}(4)-\mathrm{Si}(1)-\mathrm{Cl}(1)$ 90.11(5), N(1)-Si(1)-Cl(1) 94.33(5), N(2)-Si(1)-Cl(1) 86.36(4), N(3)-Si(1)-C(1) 105.18(6), N(4)-Si(1)-C(1) 151.39(6), N(1)-Si(1)-C(1) 35.35(5), N(2)-Si(1)-C(1) 34.78(5), $\mathrm{Cl}(1)-\mathrm{Si}(1)-\mathrm{C}(1) 88.15(4), \mathrm{N}(2)-\mathrm{C}(1)-\mathrm{N}(1)$. 


\subsubsection{Reaction with biphenylbisulfide}

Reaction of silylene with many sulfur containing moieties are known e.g. $\mathrm{CS}_{2}, \mathrm{~S}_{8}$, and PhNCS. ${ }^{56}$ However there is no report available of silylene reacting with biphenylbisulfide. The bisulfide bond (S-S) is covalent in nature with the bond dissociation energy of 60 $\mathrm{kcal} / \mathrm{mole}$. The S-S bond is weaker when compared with that of a C-C bond ${ }^{27}$ and thus susceptible to scission by polar reagents, both electrophiles and especially nucleophiles. Treatment of silylene 18 with biphenylbisulfide results in the cleavage of the S-S bond with formation of product $\mathbf{3 1}$.

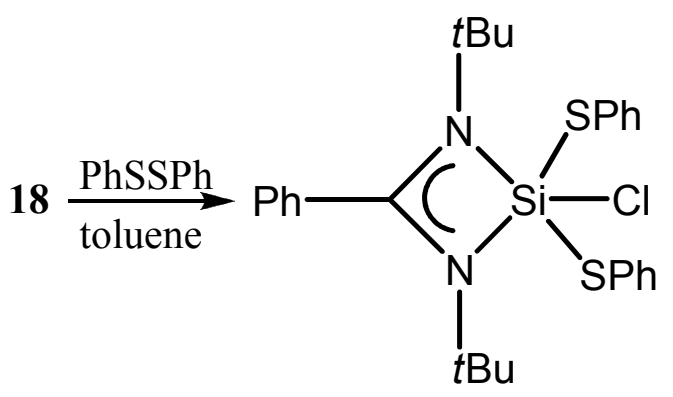

31

Scheme 2.4.2. Preparation of 31

Addition of biphenylbisulfide to in the solution of $\mathbf{1 8}$ in toluene at moderate temperature under overnight stirring gave a colorless solution. Evaporation of the solvent under vacuum yielded 31 as a colorless solid (Scheme 2.4.2). 31 is soluble in toluene and THF. The composition of $\mathbf{3 1}$ was also established by NMR spectroscopy, EI-mass spectrometry and elemental analysis. The ${ }^{1} \mathrm{H}$ NMR spectrum exhibits a resonance ( $\left.\delta 1.42 \mathrm{ppm}\right)$ which corresponds to the eighteen $t \mathrm{Bu}$ protons. The resonance for fifteen phenyl protons appears in the range of $\delta 6.90-7.91 \mathrm{ppm}$. The formation of $\mathbf{3 1}$ is accompanied by a large shift ( $\Delta \delta 97.66$ $\mathrm{ppm})$ in the ${ }^{29} \mathrm{Si}$ NMR spectrum $(\delta-83.26 \mathrm{ppm})$ compared to 18. The value is consistent with those of reported five- coordinate silicon compounds. ${ }^{43}$ In the EI-MS spectrum a peak was 
observed as the most abundant ion at $\mathrm{m} / \mathrm{z} 477$ that corresponds to a fragment after elimination of one chlorine atom from the molecular ion.

\subsubsection{Reaction of $\mathrm{LSiCl}$ with adamantylphosphaalkine}

Phosphaalkine has appeared as a new functional group in heteroatom chemistry due to its polarity, unsaturation, and high reactivity. ${ }^{57}$ Moreover the chemistry of silylene with $\mathrm{RC} \equiv \mathrm{P}$ is hitherto unknown. We reported the first successful reactivity of silylene with $\mathrm{AdC} \equiv \mathrm{P}(\mathrm{Ad}=$ adamantyl) and synthesis of a novel four-membered silicon-carbon-phosphorus ring with a bridging naked phosphorus atom of coordination number 2 that resulted from the cleavage of the $\mathrm{C} \equiv \mathrm{P}$ bond.

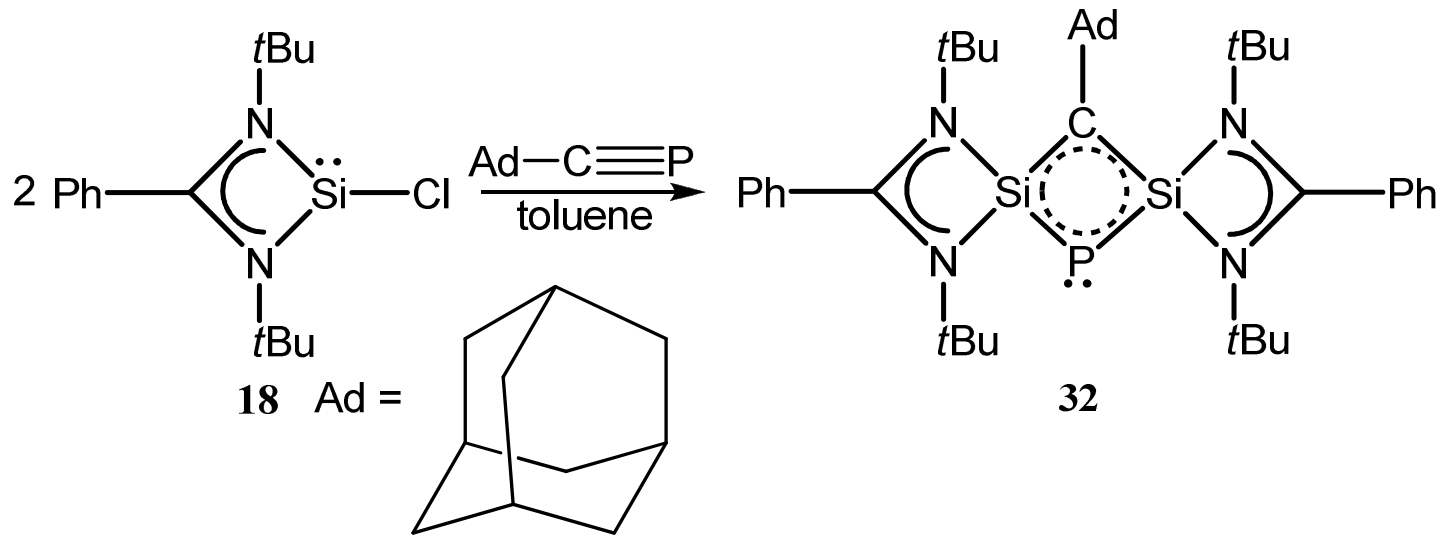

Scheme 2.4.3. Synthesis of 32

The synthesis of $\mathbf{3 2}$ is very straightforward. Addition of toluene solution of $\mathrm{AdC} \equiv \mathrm{P}$ to the toluene solution of $\mathbf{1 8}$ at $-30{ }^{\circ} \mathrm{C}$ in $1: 3$ molar ratio resulted in the formation of $\mathbf{3 2}$ which is isolated in moderate yield as reddish-yellow air- and moisture sensitive crystals upon concentrating the mother liquor (Scheme 2.4.3). Interestingly the formation of $\mathbf{3 2}$ is observed regardless of the molar ratio of the reaction partners. The product is stable under an inert atmosphere and soluble in toluene, diethyl ether, and THF. 32 was unequivocally characterized by single crystal X-ray diffraction. ${ }^{44}$ Figure 2.3 .8 depicts the molecular view of 
32 while selected bond lengths and bond angles are provided in the legend of Figure 2.3.8. 32 crystallizes in the orthorhombic space group $P 2{ }_{1} 2_{1} 2_{1}$. The main structure consists of two silicon atoms, one carbon, and one phosphorus atom, which all together form a novel $\mathrm{CSi}_{2} \mathrm{P}-$ four-membered ring. The predominant structural feature of $\mathbf{3 2}$ is the substituent free ("naked") bridged phosphorus atom, which connects the two silicon atoms. The two silicon atoms are four-coordinate and display a distorted tetrahedral geometry by coordination with two nitrogen atoms from the amidinato ligand. The remaining two sites of the tetrahedron are occupied by the carbon and the phosphorus atom. The bond lengths of the $\mathrm{P}$ atom to the atoms $\mathrm{Si}(1)$ and $\mathrm{Si}(2)$ are $2.19(7)$ and $2.20(7) \AA$, respectively, with an Si-P-Si angle of $68.86^{\circ}$. The bond length values are in between the average $\mathrm{Si}-\mathrm{P}$ single bond distance of $2.25 \AA$ and $\mathrm{Si}=\mathrm{P}$ double bond length of $2.11 \AA^{58}$ Moreover the $\mathrm{Si}(2)-\mathrm{C}(31)$ and $\mathrm{Si}(1)-\mathrm{C}(31)$ distances $(1.780$ (18) $\AA$ and $1.783(18) \AA$ ) are close to each other and significantly shorter than the Si-C single bond length reported in literature $(1.86 \AA-1.93 \AA)^{59}$ and slightly longer than that of the Si-C double bond found in $(\mathrm{TMS})_{2} \mathrm{Si}=\mathrm{C}(\mathrm{OTMS}) \mathrm{Ad}(1.764 \AA){ }^{12}$ This can be interpreted in terms of delocalization of electron throughout the $\mathrm{CSi}_{2} \mathrm{P}$-four-membered ring. Here it is noteworthy that the Si-C bond lengths in 32 matches excellently with that of Sekiguchi's 1,2disilabenzene derivative $(1.80 \AA$ and $1.79 \AA) .{ }^{60}$ Presumably the planar geometry in $\mathbf{3 2}$ is also accountable for shortening of $\mathrm{Si}-\mathrm{C}$, and $\mathrm{Si}-\mathrm{P}$ bonds. The geometry of three-coordinate $\mathrm{C}(31)$ can be best described as distorted trigonal planar. The sum of the bond angle around $\mathrm{C}(31)$ is $359.96^{\circ}$. Phosphorus too adopts a distorted trigonal planar geometry. The two Si atoms fill the two sites whereas the remaining site is occupied by the lone pair of electrons. A similar type of naked phosphorus atom is very unusual in literature. ${ }^{61}$ Another interesting feature is the $\mathrm{Si} \cdot$...Si distance $(2.48 \AA$ ), which proved that there is no Si-Si bond in 32. Here it is also noticeable that compound $\mathbf{3 2}$ contains a chain of three four-membered rings and in the spirocyclic structure each Si atom is part of two four-membered rings. 


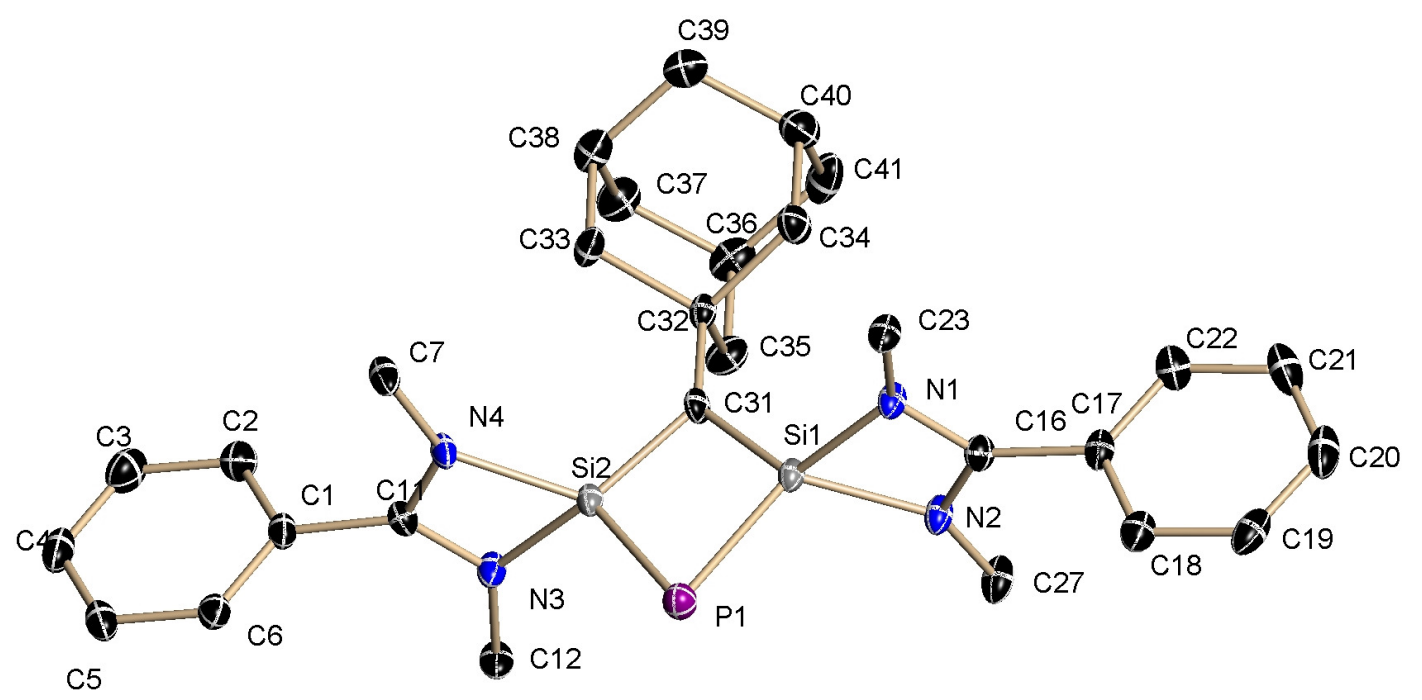

Figure 2.3.8. X-ray structure of 32. Hydrogen atoms are not shown for clarity. Selected bond distances $(\AA)$ and bond angles (deg) $\mathrm{N}(1)-\mathrm{Si}(1)$ 1.8646(17), N(2)-Si(1) 1.8653(18), N(3)-Si(2) 1.8588(18), N(4)-Si(2) 1.8624(18), $\mathrm{P}(1)-\mathrm{Si}(2)$ 2.1894(7), $\mathrm{P}(1)-\mathrm{Si}(1)$ 2.2012(7), $\mathrm{Si}(1)-\mathrm{C}(31)$ 1.7808(18), $\mathrm{Si}(2)-\mathrm{C}(31) 1.7834(18) ; \quad \mathrm{Si}(2)-\mathrm{P}(1)-\mathrm{Si}(1) \quad 68.86(3), \quad \mathrm{C}(31)-\mathrm{Si}(1)-\mathrm{N}(1) \quad$ 124.05(9), $\mathrm{C}(31)-\mathrm{Si}(1)-\mathrm{N}(2)$ 124.15(9), N(1)-Si(1)-N(2) 69.95(7), C(31)-Si(1)-P(1) 101.23(6), N(1)-Si(1)$\mathrm{P}(1)$ 117.63(6), $\mathrm{N}(2)-\mathrm{Si}(1)-\mathrm{P}(1)$ 119.32(7), $\quad \mathrm{C}(31)-\mathrm{Si}(2)-\mathrm{N}(3)$ 122.57(9), $\mathrm{C}(31)-\mathrm{Si}(2)-\mathrm{N}(4)$ 121.70(9), $\quad \mathrm{N}(3)-\mathrm{Si}(2)-\mathrm{N}(4) \quad 70.27(7), \quad \mathrm{C}(31)-\mathrm{Si}(2)-\mathrm{P}(1) \quad 101.59(6), \quad \mathrm{N}(3)-\mathrm{Si}(2)-\mathrm{P}(1)$ 119.44(7), N(4)-Si(2)-P(1) 120.78(7)

Additionally, 32 was characterized by NMR spectroscopy, EI-MS spectrometry, and elemental analysis. The NMR spectroscopic data are in accord with the constitution of $\mathbf{3 2}$ as derived from X-ray structural analysis. In the ${ }^{1} \mathrm{H}$ NMR four sets of resonances are observed. The $t \mathrm{Bu}$ protons appear at $\delta 1.26 \mathrm{ppm}$, which are shifted downfield compared to those of $\mathbf{1 8}(\delta$ $1.08 \mathrm{ppm})$. Hydrogens attached to tertiary carbon of the adamantyl group appear at $\delta 1.21$ ppm, whereas hydrogens attached to secondary carbons exhibit a resonance at $\delta 1.72 \mathrm{ppm}$. The phenyl protons of the amidinato ligand display a resonance at $\delta 7.45 \mathrm{ppm}$. Along with it two sets of resonances are also found in the ${ }^{1} \mathrm{H}$ NMR at $\delta 1.17 \mathrm{ppm}$ and $\delta 6.68-6.85 \mathrm{ppm}$. These resonances indicate the formation of $\mathrm{LSiCl}_{3}$ as a minor product. The ${ }^{1} \mathrm{H}$-coupled as 
well as the ${ }^{1} \mathrm{H}$-decoupled ${ }^{31} \mathrm{P}$ NMR spectrum of $\mathbf{3 2}$ shows a sharp singlet at $-243 \mathrm{ppm}$, which can be assigned to the naked $\mathrm{P}$ atom. In the ${ }^{29} \mathrm{Si}$ NMR spectrum a sharp resonance exhibits at $\delta-5.1 \mathrm{ppm}$ with the coupling constant $J_{\mathrm{Si}-\mathrm{P}}=75 \mathrm{~Hz}$ which is remarkably low compared to the Si-P coupling constants found in literature. ${ }^{58}$ The formation of $\mathrm{LSiCl}_{3}$ as a side product is justified from ${ }^{29} \mathrm{Si}$ NMR because a sharp resonance is detected at $\delta-98.4 \mathrm{ppm}$, which corresponds to the penta-coordinate silicon atom of $\mathrm{LSiCl}_{3}{ }^{25 \mathrm{a}}$ When the reaction has been carried out in 1:1 or 2:1 molar ratio, the same products are obtained along with excess of $\mathrm{AdC} \equiv \mathrm{P}$, which is indicated in the ${ }^{31} \mathrm{P}$ NMR spectrum $(\delta-68.4 \mathrm{ppm})$ of the crude product. In the EI-MS spectrum the molecular ion is observed as the most abundant peak with highest relative intensity at $m / z 696$.

\subsubsection{Reaction of LSiCl with metal carbonyls}

Transition-metal silylene complexes are of great interest not only due to their intriguing bonding nature between transition metals and silicon, but their similarity to transition-metal carbene complexes, while the latter serve as extremely fruitful catalysts for many organic transformations. ${ }^{62}$ In organosilicon chemistry these silylene metal complexes are postulated as probable catalytic intermediates in a number of metal-catalyzed silylene transfer reactions. ${ }^{63}$ In 1987, Tilley and coworkers reported on two base stabilized silylene complexes $(\mathrm{CO})_{4} \mathrm{FeSi}(\mathrm{O} t \mathrm{Bu})_{2}\left\{(\mathrm{O}) \mathrm{P}\left[\mathrm{NMe}_{2}\right]_{3}\right\} \quad$ and $\quad\left\{\mathrm{Cp} *\left[\mathrm{Me}_{3} \mathrm{P}\right]_{2} \mathrm{RuSiPh}_{2}[\mathrm{MeCN}]\right\} .^{64}$ These two complexes were synthesized by the indirect salt elimination and by triflate abstraction method. Instead of the aforesaid method, another promising alternative route to prepare metalsilylene complexes is the utilization of N-heterocyclic silylenes (NHSis). The discovery and elucidation of the first NHSi by West is considered as a landmark in organosilicon chemistry. ${ }^{22}$ A number of interesting chemical compounds and applications have stemmed from this discovery, ${ }^{65}$ which challenged long lasting traditional notions. As there is significant $p$-electron donation from the adjacent nitrogen lone pairs into empty $p$ orbitals on silicon, 
which leads to a strong stabilizing of the NHSis, these system can be considered as strong $\sigma$ donors and weak $\pi$ acceptors. ${ }^{66}$

In 1994 West and co-workers isolated $\mathrm{Ni}(\mathrm{CO})_{2}(\mathrm{NHSi})_{2}[\mathrm{NHSi}=(t \mathrm{BuNCH}=\mathrm{CHN} t \mathrm{Bu})]$ from the reaction of $\mathrm{NHSi}$ with $\mathrm{Ni}(\mathrm{CO})_{4}$ in a molar ratio of $2: 1 .^{67}$ The success of this reaction enthroned silylene as the pre-eminent ligand in transition metal chemistry and established the concept that NHSis may resemble phosphines. Since then, there is a burgeoning interest in the reaction of stable silylenes with transition metals, ${ }^{68-71}$ because the study of catalytic properties of silylene-metal complexes will be exciting as carbene transition metal complexes served as powerful catalysts for several organic reactions, and moreover such compounds may also be studied as precursors for preparing silicon-metal alloys by chemical vapor deposition. ${ }^{56 a}$

\section{Reaction of $\mathrm{LSiCl}$ with $\mathrm{Ni}(\mathrm{CO})_{4}$ and $\mathrm{Fe}_{2}(\mathrm{CO})_{9}$}

To probe the reaction of three-coordinate heteroleptic silylene with metal carbonyls, we reacted 18 with $\mathrm{Ni}(\mathrm{CO})_{4}$ and $\mathrm{Fe}_{2}(\mathrm{CO})_{9}$. The reactions of 18 with $\mathrm{Ni}(\mathrm{CO})_{4}$ and $\mathrm{Fe}_{2}(\mathrm{CO})_{9}$ respectively were straight forward, which afforded silylene carbonyl complexes $\mathbf{3 3}$ and $\mathbf{3 4}$ (Scheme 2.4.4). In the former case one carbonyl group was displaced, whereas in the latter case $\mathrm{Fe}(\mathrm{CO})_{5}$ was liberated. Both products are extremely air- and moisture sensitive and immediately decompose when exposed to air. They are well soluble in solvents like diethyl ether, toluene, and THF.

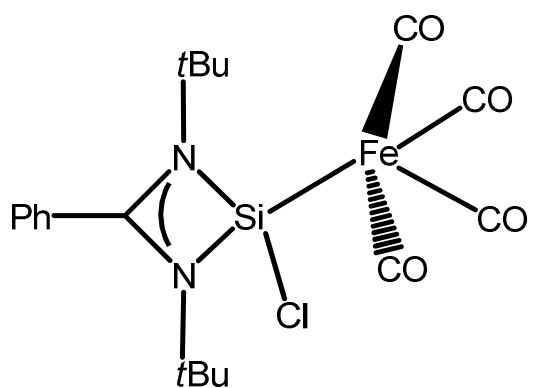

34

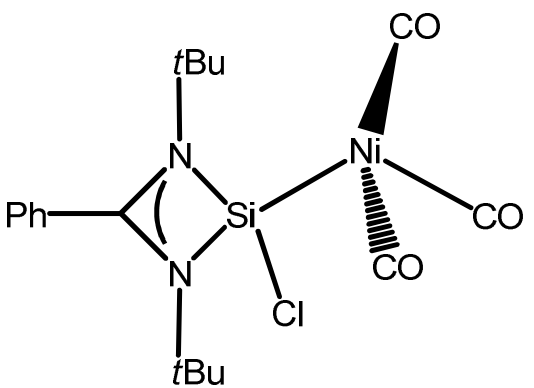

33

Scheme 2.4.4. Synthesis of silylene metal carbonyl complexes of 33 and 34 
The coordination of the metal to silicon resulted in a downfield chemical shift in the ${ }^{29} \mathrm{Si}$ NMR spectrum. The silylene-nickel complex of $\mathbf{3 3}$ resonates at $\delta 62.69 \mathrm{ppm}\left({ }^{29} \mathrm{Si} \mathrm{NMR}\right.$ of 18: $14.16 \mathrm{ppm}$ ), while in $\mathbf{3 4}$ it is observed at $\delta 43.19 \mathrm{ppm}$. The downfield chemical shift is due to the deshielding upon coordination of the metal atom to silicon. These values are consistent with those reported for base stabilized silylene transition metal complexes $(\delta 40.30$ ppm for $\mathrm{Ph}(\mathrm{N} t \mathrm{Bu})_{2} \mathrm{SiO} t \mathrm{Bu} \cdot \mathrm{Fe}(\mathrm{CO})_{4}$ and $\delta 44.25 \mathrm{ppm}$ for $\left(\mathrm{Co}(\mathrm{CO})_{3}\left\{\mathrm{SiCl}_{2} \mathrm{R}\right\}_{2}^{+}\left[\mathrm{CoCl}_{3}(\mathrm{THF})\right]^{-}\right)$ $\mathrm{R}=1,3$-bis-(2,6- $\left.i \mathrm{Pr}_{2} \mathrm{C}_{6} \mathrm{H}_{3}\right)$ imidazol-2-ylidene). ${ }^{69,70}$

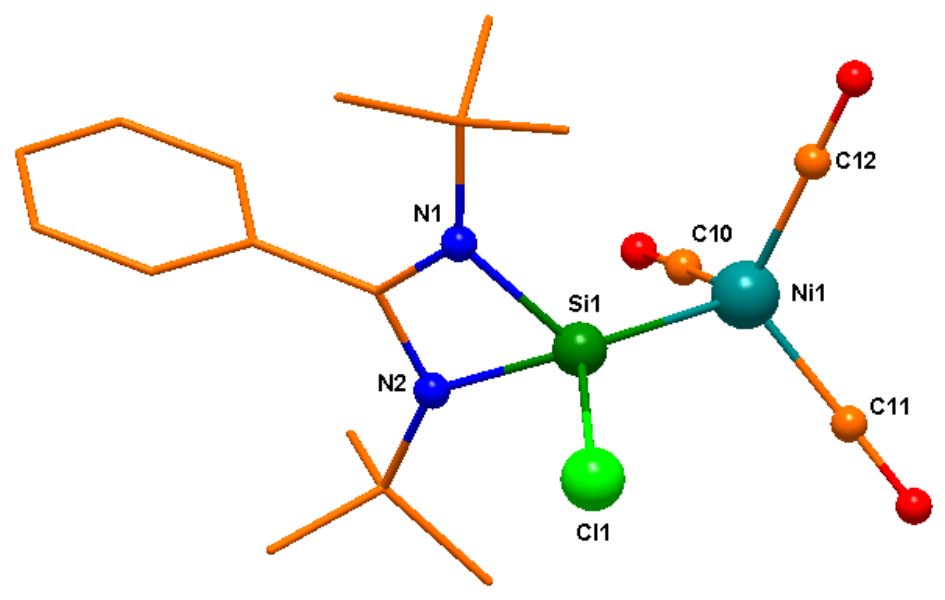

Figure 2.3.9. Molecular Structure of 33. Hydrogen atoms are removed for clarity. Selected bond lengths $(\AA)$ and angles (deg) Ni1-Si1 2.2111(8), Ni1-C10 1.795(2), Ni1-C11 1.7967(19), Ni1-C12 1.798(2), Cl1-Si1 2.1149(7), Si1-N1 1.8456(15), Si1-N2 1.8373(15); C10-Ni1-C11 114.39(9), C10-Ni1-C12 114.47(9), C11-Ni1-C12 110.48(8), C10-Ni1-Si1 102.67(7), C11Ni1-Si1 105.66(6), C12-Ni1-Si1 108.31(6), N2-Si1-N1 71.15(7), N2-Si1-Cl1 102.23(5), N1Si1-Cl1 102.24(5), N2-Si1-Ni1 123.18(5), N1-Si1-Ni1 125.63(5), Cl1-Si1-Ni1 120.87(3), N2Si1-C26 35.72(6), N1-Si1-C26 35.81(6), Cl1-Si1-C26 109.08(5).

The molecular structures of $\mathbf{3 3}$ are given in Figure 2.3.9. 33 crystallizes in the monoclinic space group $P 2_{1} / c{ }^{44}$ The $\mathrm{Si}-\mathrm{Ni}$ bond length in $\mathbf{3 3}$ is $2.211(8) \AA$ and quite comparable to those of the other silylene nickel complexes reported in literature. ${ }^{67,71}$ The Si-Ni bond in $\mathbf{3 3}$ is 0.14 $\AA$ shorter than the sum of the covalent radii of $\mathrm{Si}(1.11 \AA$ ) and Ni (1.24 $\AA$ ). A similar decrease in bond length is also observed in the $\mathrm{Si}$-Fe bond in $\mathrm{Ph}(\mathrm{N} t \mathrm{Bu})_{2} \mathrm{SiO} t \mathrm{Bu} \cdot \mathrm{Fe}(\mathrm{CO})_{4}(2.23 \AA)$ complex, ${ }^{69}$ which is $0.2 \AA$ shorter than the sum of the covalent radii of $\mathrm{Si}(1.11 \AA)$ and Fe 
$\left(1.32 \AA\right.$ ) and $\mathrm{Si}-\mathrm{Co}$ bond $(2.22 \AA)$ in $\left.\left[\mathrm{Co}(\mathrm{CO})_{3}\left\{\mathrm{SiCl}_{2} \mathrm{R}\right\}_{2}\right]^{+}\left[\mathrm{CoCl}_{3}(\mathrm{THF})\right]^{-}\right){ }^{70}$ which is $0.15 \AA$ shorter compared to the sum of covalent radii of silicon and cobalt. These results indicate some possible $\pi$ - back bonding within the Ni-Si bond. There is a slight change in the Ni-C bond lengths when compared with that of the precursor. The average Ni-C bond lengths in $\mathrm{Ni}(\mathrm{CO})_{4}$ is $1.817(2) \AA{ }^{66}$ whereas in $\mathbf{3 3}$ the $\mathrm{Ni}-\mathrm{C}_{\mathrm{av}}$ bond distance is $1.796(2) \AA$.The silicon atom in $\mathbf{3 3}$ is four-coordinate $(2 \mathrm{~N}, 1 \mathrm{Cl}, 1 \mathrm{Ni})$ and adopts a distorted tetrahedral geometry. The bond length of $\mathrm{Si}-\mathrm{Cl}$ in $\mathbf{3 3}$ is $2.1146(121) \AA$ [ $\mathrm{Si}-\mathrm{Cl}$ of $\mathbf{1 8}$ is $2.156(1) \AA$ ] and the cone angle $(\angle \mathrm{N}-\mathrm{Si}-\mathrm{N})$ is $71.210(261)^{\circ}\left[\angle \mathrm{N}-\mathrm{Si}-\mathrm{N}\right.$ of $\mathbf{1 8}$ is $\left.68.35(8)^{\circ}\right]$.

\subsection{Conclusion}

We have reported two new approaches for synthesizing $\mathrm{LSiCl}$, $\left(\mathrm{L}=\mathrm{PhC}(\mathrm{N} t \mathrm{Bu})_{2}\right)$. The reaction of $\mathrm{LSiHCl}_{2}$ with bis-trimethylsilyl lithium amide and $\mathrm{N}$-heterocyclic carbene afforded 18 respectively. In the former method silylene is produced in 90\% yield. Furthermore we have prepared a variety of silicon heterocycles by treating $\mathbf{1 8}$ with a different type of unsaturated organic compounds. The experimental data for the reactions of $\mathbf{1 8}$ disclose the proclivity of $\mathbf{1 8}$ towards oxidative addition due to the presence of the stereochemically active lone pair of electrons on the silicon center. This is in good agreement with the reactivity of previously reported silylenes. In addition we showed that $\mathbf{1 8}$ is quite versatile for the synthesis of silylene metal carbonyl complexes. The nature of the ligand dictates the displacement of carbonyl groups from the $\mathrm{Ni}(\mathrm{CO})_{4}$. The above versatile and diverse nature of 18 can be utilized as a scaffold for the preparation of other different transition metal silylene complexes. Moreover one can also pursue the reactivities of $\mathbf{3 3}$ with reactive $\mathrm{Si}-\mathrm{Cl}$ bond after coordinating to the lone pair of electrons, which will provide synthetic access to other fascinating silicon compounds. 


\section{Chapter 3}

\section{A Remarkable Base Stabilized Bis-Silylene with a}

\section{$\mathrm{Si}(\mathrm{I})-\mathrm{Si}(\mathrm{I})$ Single Bond}

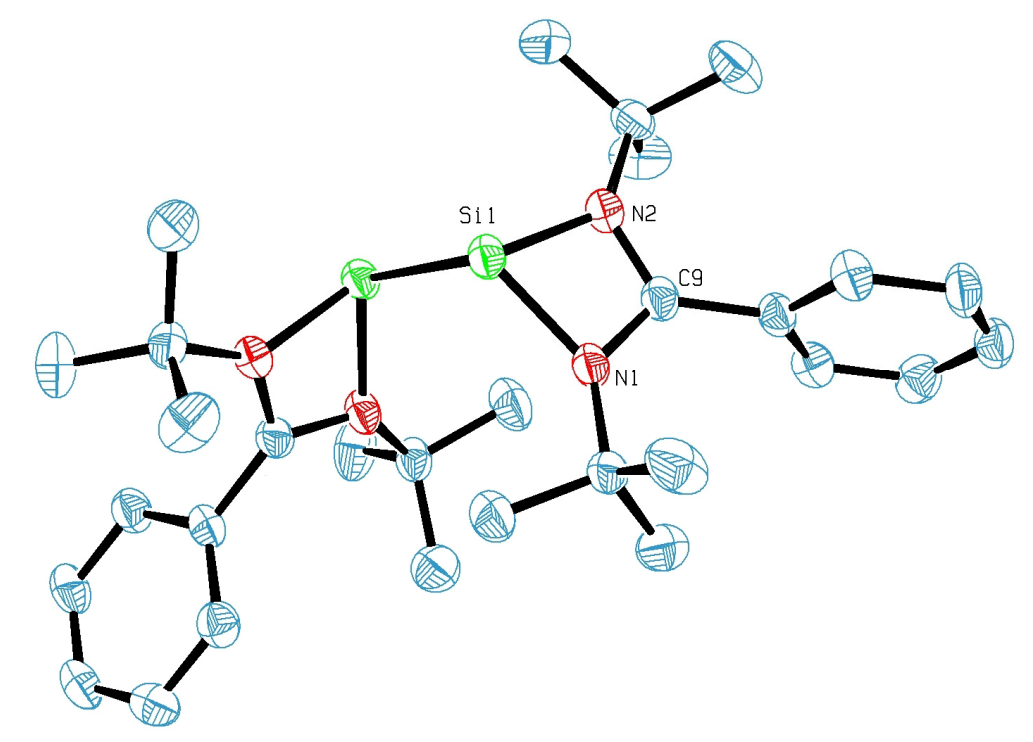

\section{Abstract}

A compound with a $\mathrm{Si}(\mathrm{I})-\mathrm{Si}(\mathrm{I})$ single bond was prepared by the reduction of amidinato chlorosilane with potassium graphite. There is no multiple bond character in the Si-Si bond. Moreover the X-ray structural analysis and the theoretical calculation show that this bissilylene possesses a gauche-bent geometry instead of the usual trans-bent arrangement. 


\subsection{Introduction}

The most striking characteristic of the bond that holds atoms together in a metallic aggregate is the mobility of the bonding electrons...

(Linus Pauling, The Nature of the Chemical Bond, 3rd ed.; Cornell University Press: Ithaca, NY, 1960; p 10). This is the statement from perhaps the most significant chemistry text of this century, written by the discipline's most influential and celebrated practitioner. As manifested by the spectacular range and diversity of organometallic compounds containing metal-metal bonds, which have already been brought to the fore, the austere insight of Pauling is all the more poignant with the passing of nearly five decades.

After being successful in studying the preparation of silylene and its reactivity we were curious to investigate the nature of the Si-Si bonding. Double and triple covalent bonds are ubiquitous in carbon chemistry and have been studied for more than two centuries but were unusual with its congener in the periodic table, silicon. The initial attempts to synthesize such compounds were unsuccessful, resulting in the formation of polymeric substances. This changed when West and coworkers in 1981 synthesized a compound containing a $\mathrm{Si}-\mathrm{Si}$ double bond $\left(\mathrm{R}_{2} \mathrm{Si}=\mathrm{SiR} 2, \mathrm{R}=\mathrm{Me}_{3} \mathrm{C}_{6} \mathrm{H}_{2}\right.$ ), where each $\mathrm{Si}$ atom has a formal oxidation state of + II. $^{11}$ Key to the discovery of stable compounds containing Si-Si double bonds was the protection of the double bonds by bulky substituents, which provide kinetic stability. Apeloig and co-workers showed that silylene has a singlet ground state and the ${ }^{3} \mathrm{~B}_{1}$ triplet state is lying significantly higher in energy. ${ }^{72}$ Moreover recent calculations exhibit that the energy difference between the singlet and triplet state of silylene is around $18-21 \mathrm{Kcal} / \mathrm{mol}{ }^{73}$ This singlet-triplet energy difference of the silylene fragments is the main reason for the weakness of the $\mathrm{Si}=\mathrm{Si}$ double bond. This now generally accepted model originated from Carter, Goddard, Malrieu, and Trinquier (CGMT), who described the double bond topology as being 
a function of the energy difference between the singlet and the triplet state of the carbene-like fragments formally constituting the double bond. ${ }^{74}$ In 2004 , Sekiguchi and coworkers and Wiberg et al. were successful of isolating a compound containing a $\mathrm{Si}-\mathrm{Si}$ triple bond $\left(\mathrm{RSi} \equiv \mathrm{SiR} ; \mathrm{R}\right.$ are $\mathrm{Si}(i \mathrm{Pr})\left\{\mathrm{CH}\left(\mathrm{SiMe}_{3}\right)_{2}\right\}_{2}{ }^{19}$ and $\mathrm{SiMe}\left(\mathrm{Si}_{\mathrm{B}} \mathrm{Bu}_{3}\right)_{2}{ }^{75}$ respectively) where the formal oxidation state of $\mathrm{Si}$ is $+\mathrm{I}$. Following this, Robinson and coworkers synthesized two compounds, one with a Si-Si single bond having formal oxidation state one and another with a $\mathrm{Si}-\mathrm{Si}$ double bond where the formal oxidation state of $\mathrm{Si}$ is zero ( $\mathrm{RClSi}-\mathrm{SiClR}$ and $\mathrm{RSi}=\mathrm{SiR}, \mathrm{R}=1,3$-bis-(2,6-diisopropylphenyl)imidazol-2-ylidene ). ${ }^{21}$ The former compound was stabilized by an N-heterocyclic carbene and one chlorine atom was attached to each silicon center making the formal oxidation state of silicon $+\mathrm{I}$. This is a unique property because each Si center, which features a lone pair of electrons is simultaneously involved in bonding. These two attributes are usually associated with extreme instability. In view of this we became interested in synthesizing one compound with a $\mathrm{Si}-\mathrm{Si}$ single bond stabilized by a monoanionic ligand and avoiding the lone pair of electrons taking part in any bonding. We were recently successful in using an amidinate ligand with $t \mathrm{Bu}$ substituents on the nitrogen atom in stabilizing heteroleptic silylenes. It seems, that such a ligand may also stabilize a Si(I) compound with a $\mathrm{Si}(\mathrm{I})-\mathrm{Si}(\mathrm{I})$ single bond.

\subsection{Synthesis and Characterization of $\left[\mathrm{PhC}(\mathrm{N} t \mathrm{Bu})_{2}\right]_{2} \mathrm{Si}_{2}$}

The reaction of tert-butylcarbodiimide with one equivalent of $\mathrm{PhLi}$ in diethyl ether followed by treatment with $\mathrm{SiCl}_{4}$ afforded $\left[\mathrm{PhC}(\mathrm{N} t \mathrm{Bu})_{2}\right] \mathrm{SiCl}_{3}$ (21a; Scheme 3.1). Treatment of 21a with 3 equivalents of potassium graphite $\left(\mathrm{KC}_{8}\right)$ in THF afforded air-sensitive, orange-red crystals of $\mathbf{3 5}$ (5.21\% yield). $\mathbf{3 5}$ is highly soluble in solvents like diethyl ether, toluene and THF. It has been characterized by elemental analysis, spectroscopic methods, and X-ray structural analysis. The ${ }^{1} \mathrm{H}$ and ${ }^{13} \mathrm{C}$ NMR spectra of 35 (in THF- $d_{8}$ ) display one set of resonances that result from the amidinate ligand. The resonances show a downfield shift 
relative to that of $\mathbf{2 1 a}$ and also from $\mathbf{1 8} .^{25 a}$ The shift is probably due to the lower oxidation state of the silicon center $+\mathrm{I}$ in $\mathbf{3 5}$ compared to silylene, where normally a formal oxidation state of $\mathrm{Si}$ is $+\mathrm{II}$ observed. The ${ }^{29} \mathrm{Si}$ NMR resonance of $\mathbf{3 5}$ (in THF- $\left.d_{8}\right),(\delta 75.71 \mathrm{ppm}$ ) is more downfield compared to that of RClSi-SiClR, $(38.4 \quad \mathrm{ppm}) \quad[\mathrm{R}=1,3-\mathrm{bis}-(2,6-$ diisopropylphenyl)imodazol-2-ylidene]. ${ }^{21}$ We were also curious to measure the NMR in nonpolar solvent $\left(\mathrm{C}_{6} \mathrm{D}_{6}\right)$. In the ${ }^{29} \mathrm{Si}$ NMR 35 resonates at $76.29 \mathrm{ppm}$. So, virtually there is very little downfield shift in the ${ }^{29} \mathrm{Si}$ NMR. The molecular ion of $\mathbf{3 5}$ appeared as the most abundant peak in the EI-MS spectrum at $m / z$ 518.3.

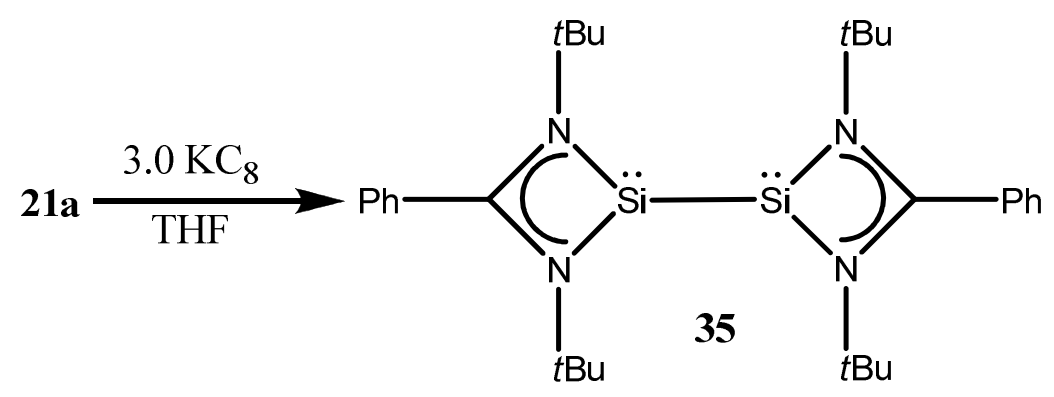

Scheme 3.1. Preparation of $\mathbf{3 5}$

The molecular structure of $\mathbf{3 5}$ is shown in Figure $3.1 .^{44}$ Compound $\mathbf{3 5}$ crystallizes in the monoclinic space group $C 2 / c$. The coordination environment of each of the $\mathrm{Si}(\mathrm{I})$ atoms exhibits a distorted tetrahedral geometry. The coordination sites of the $\mathrm{Si}(\mathrm{I})$ centers are each occupied by the $\mathrm{N}$ atoms of the amidinato ligand and by the other $\mathrm{Si}(\mathrm{I})$ atom. The lone pair of the $\mathrm{Si}(\mathrm{I})$ occupies the remaining coordination site of the tetrahedral geometry. 


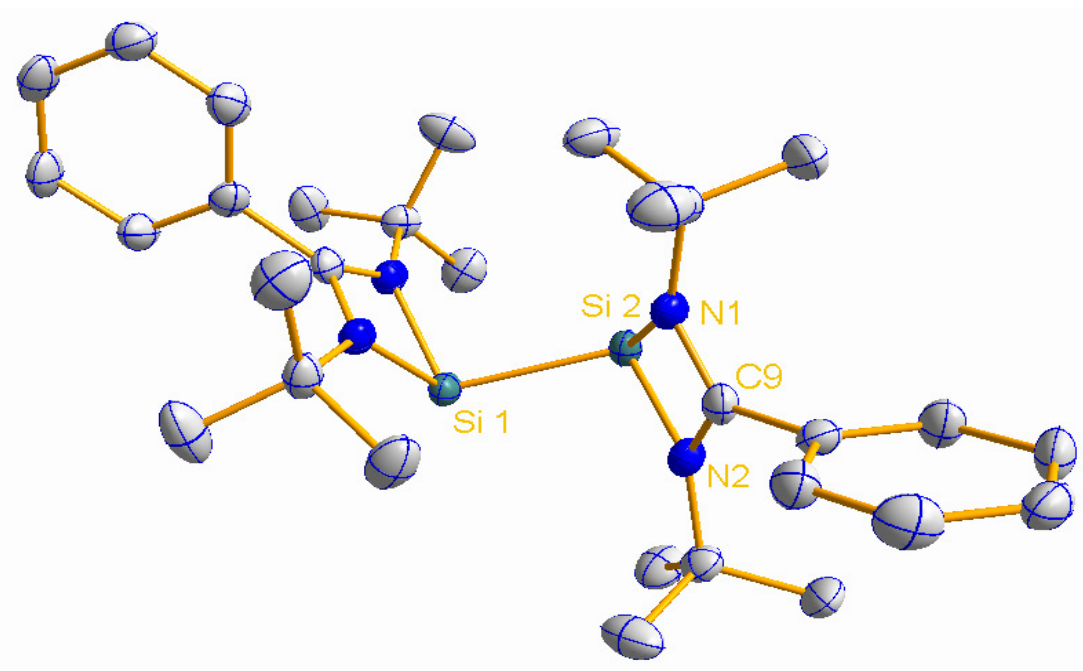

Figure 3.1 ORTEP view (50\% ellipsoid probability) of compound 35. Hydrogen atoms have been removed for clarity. Selected bond distances $(\AA)$ and bond angles (deg) $\mathrm{Si}(1)-\mathrm{Si}(2)$ $2.413(2), \mathrm{Si}(2)-\mathrm{N}(2) 1.866(4), \mathrm{Si}(2)-\mathrm{N}(1)$ 1.874(4), $\mathrm{C}(1)-\mathrm{N}(1)$ 1.467(6), C(9) - N(1) 1.331(6), N(2) - Si(2) - N(1) 69.52(18), N(2) - Si(2) - Si(1) 102.15(14), N(1) - Si(2) - C(1) 130.09(4).

The X-ray structural analysis of $\mathbf{3 5}$ reveals that the $\mathrm{Si}-\mathrm{Si}$ core is sterically well-shielded by the two amidinate ligands. The central Si-Si bond distance $(2.413(2) \AA$ ) is only about $0.07 \AA$ longer than the sum of the Si covalent radii $(2.34 \AA)^{76}$ and about $0.05 \AA$ longer than the $\mathrm{Si}-\mathrm{Si}$ single bond distance in $\alpha$-silicon $(2.36 \AA) .{ }^{77}$ The value is $5.17 \%$ larger than the longest disilene bond distance $(2.29 \AA)$ and $14.6 \%$ longer than the reported disilyne bond distance $(2.06 \AA)$ and also well comparable with that of the $\mathrm{Si}(\mathrm{I})$ dimer reported by Robinson et al. (2.393 $\AA$ ). The sum of the bond angles of the Si atoms in $\mathbf{3 5}\left(282.35^{\circ}\right.$ average $)$ compares very well with literature value. ${ }^{78}$ Compound 35 possesses a gauche-bent conformation. This structural feature of $\mathbf{3 5}$ is akin to the isolobal equivalent hydrazine and diphosphine. ${ }^{79}$ 


\subsection{Ab initio DFT calculation of $\left[\mathrm{PhC}(\mathrm{N} t \mathrm{Bu})_{2}\right]_{2} \mathrm{Si}_{2}$}

To obtain a better insight of the bonding characteristics of the $\operatorname{Si}(\mathrm{I})-\mathrm{Si}(\mathrm{I})$ unit in compound 35, we have performed ab initio DFT calculations at $B 3 \mathrm{LYP}^{80}$ level for exchange and correlation functional and LANL2DZ ${ }^{81}$ basis set as implemented in Gaussian 03 package. ${ }^{82}$ The positions of hydrogen atoms in the experimentally obtained structure have been relaxed keeping in mind that the X-ray crystallography cannot precisely locate the hydrogen atoms. For a structural comparison, we also performed optimization of the total molecule, and the attainment of the global minima has been confirmed by vibrational frequency analysis. The geometry of the fully relaxed structure resembles the experimentally obtained structure quite well with only little variation. Upon relaxation, the $\mathrm{Si}-\mathrm{Si}$ bond length shows an increase of $0.07326 \AA$ (2.4124 $\AA$ to $2.4856 \AA)$ and the C9 - Si1 - Si1A - C9A dihedral angle experiences an increase of $2.72834^{\circ}\left(96.46536^{\circ}\right.$ to $\left.99.1937^{\circ}\right)$. The $\mathrm{Si}-\mathrm{N}$ bonds also show an average of $\sim 3 \%$ increase.

To understand the nature of bonding, we have performed natural atomic orbital (NAO) and natural bonding orbital (NBO) analysis. It shows that the two Si centers are connected by a $\sigma$ bond, characterized by the Wiberg bond order of 0.9768 with high $p$-character $(s: 18.27 \%, p$ : $81.73 \%$ ). Interestingly, the single non-bonded electron on each Si center is found to reside in orbitals with very high $s$-character $(s: \sim 65 \%, p: \sim 35 \%)$. This is evident from the highest occupied molecular orbital (HOMO) picture in Figure 3.2(a) which shows localization of spherically symmetric orbitals on the Si centers. In fact, due to the lack of directionality of these orbitals and the constraint of bonding configuration of $\mathrm{Si}(\mathrm{I})$ in the geometry, the single electron pair rather prefers to remain non-bonded in each $\mathrm{Si}(\mathrm{I})$ center.

The lowest unoccupied molecular orbital (LUMO) on the contrary shows the $\pi^{*}$-type interaction (orthogonal lobes) between the Si centers (see Figure 3.2(b)). Interestingly the $\mathrm{Si}$ $\mathrm{N}$ bonds in the molecule are highly polarized with charge separation of 0.57 and -0.80 
(average) over $\mathrm{Si}$ and $\mathrm{N}$ centers respectively and an average Wiberg bond order of $\sim 0.5$. Additionally, considering the spin degrees of freedom explicitly, ${ }^{83}$ we found that the singlet state is preferred over the triplet state by $2.37 \mathrm{eV}$, suggesting an anti-parallel alignment of the spins on two Si atoms, as shown in Figure 3.2(c).

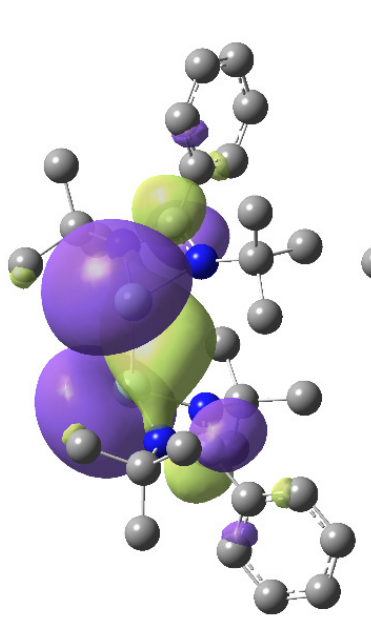

(a)

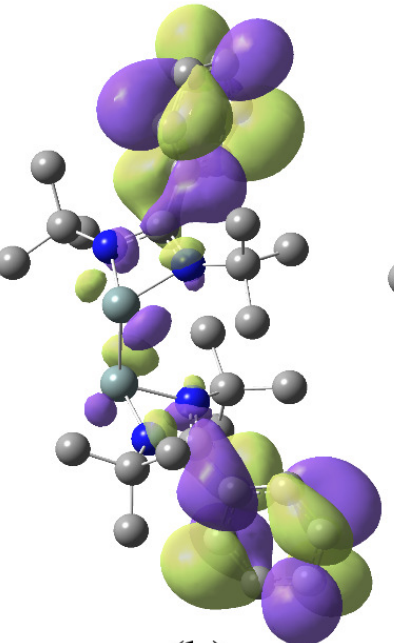

(b)

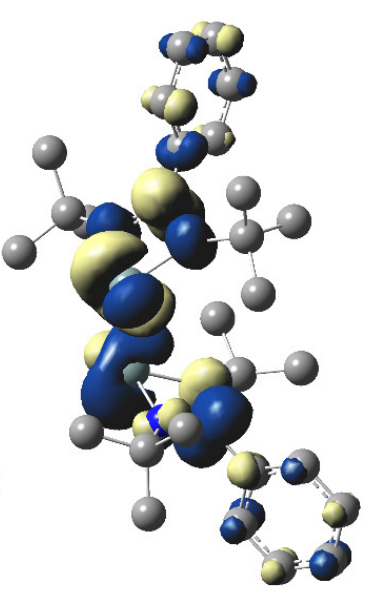

(c)

Figure 3.2 (a) HOMO, (b) LUMO and (c) Spin density plot of compound 35. For clarity, the hydrogen atoms are removed.

Also, to verify the stability of the gauche-bent structure of $\mathrm{Si}(\mathrm{I})$ - $\mathrm{Si}(\mathrm{I})$ moiety, we varied the C9 - Si1 - Si1A - C9A dihedral angle in the molecule, for the crystal geometry as well as for the theoretically fully relaxed structure. The energy vs. dihedral angle plots (see Figure 3.3) for both cases show that, the gauche-bent structure is more stable than its trans analogue, which is reflected in experimental isolation of this system. 


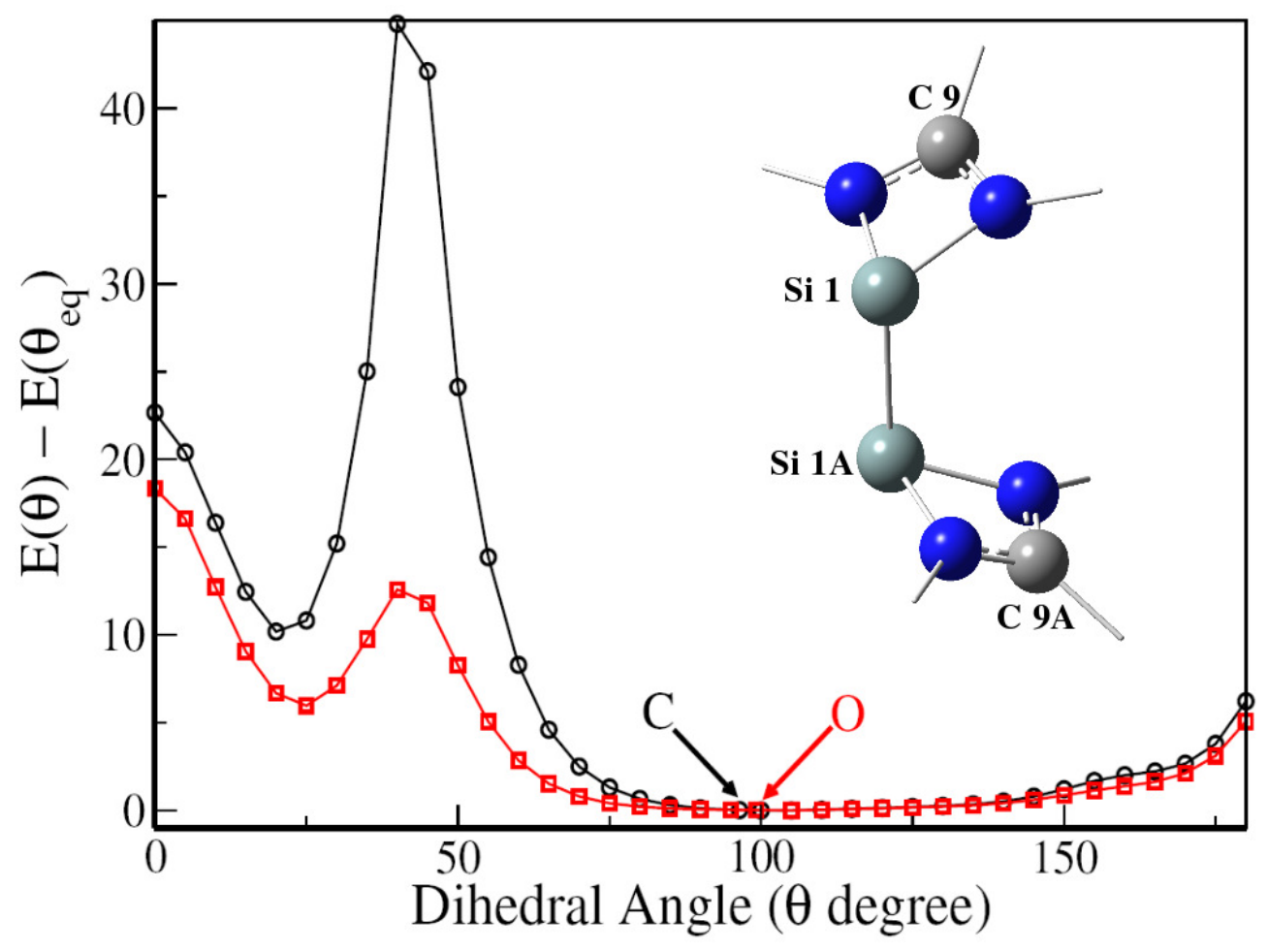

Figure 3.3. The stability analysis (in eV) of the gauche-bent structure of compound 35 as a function of the $\mathrm{C} 9-\mathrm{Si} 1-\mathrm{Si} 1 \mathrm{~A}-\mathrm{C} 9 \mathrm{~A}$ dihedral angle for both experimentally (circles) and theoretically (square) obtained structures. The "C" and "O" specify the ground state structures $\left(\right.$ at $\theta_{\text {eq }}$ ) obtained experimentally and theoretically, respectively.

\subsection{Conclusion}

In conclusion, we have prepared and fully characterized a compound with a $\mathrm{Si}(\mathrm{I})-\mathrm{Si}(\mathrm{I})$ bond, which is stabilized by bulky amidinate ligands. Theoretical studies confirm that $\mathbf{3 5}$ exhibits a gauche-bent geometry with a stereochemically active lone pair of electrons on each silicon center and the $\mathrm{Si}-\mathrm{Si}$ bond shows no multiple bond character. In the next chapter we will discuss the reactivity of $\mathbf{3 5}$. 


\section{Chapter 4}

\section{Reactions of Base Stabilized Bis-Silylene}

\section{$\left(\left[\mathrm{PhC}(\mathrm{N} t \mathrm{Bu})_{2}\right]_{2} \mathrm{Si}_{2}\right)$ with various organic substrates:}

\section{Probing the Nature of the Si-Si Bond}

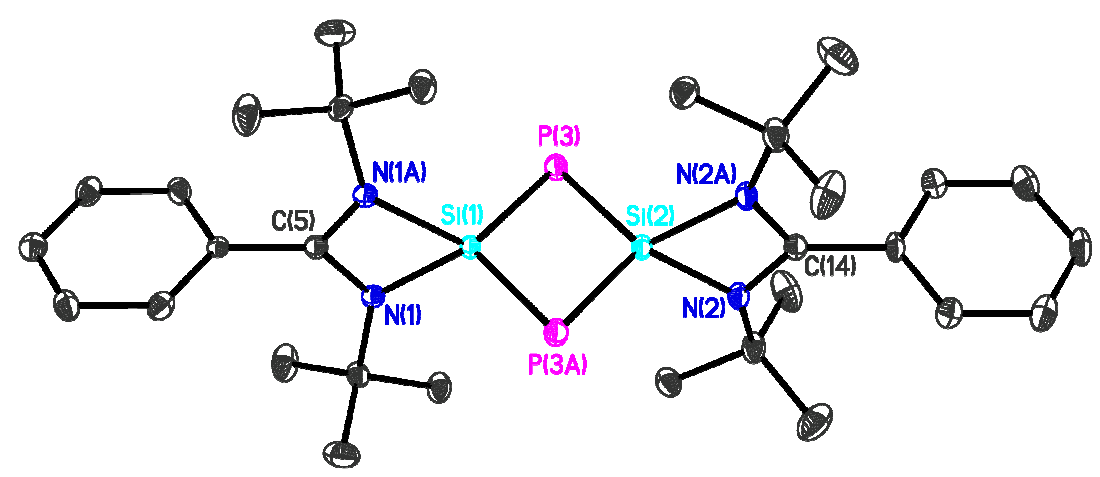

The reactivity of $\mathrm{LSi}^{\mathrm{I}}-\mathrm{Si}^{\mathrm{I}} \mathrm{L}$ with benzophenone, benzil, $\mathrm{N}_{2} \mathrm{O}$, and $t \mathrm{Bu}$-isocyanate is described here. With benzophenone the cleavage of the Si-Si bond is observed whereas with benzil the $\mathrm{Si}-\mathrm{Si}$ bond is preserved. Moreover $\mathrm{LSi}^{\mathrm{I}}-\mathrm{Si}^{\mathrm{I}} \mathrm{L}$ is treated with $\mathrm{N}_{2} \mathrm{O}$ to afford two four-membered $\mathrm{Si}_{2} \mathrm{O}_{2}$ rings connected with two oxygen atoms with the cleavage of $\mathrm{Si}-\mathrm{Si}$ bond. The identical product is obtained when the reaction is performed with $t \mathrm{Bu}$-isocyanate with the liberation of $t \mathrm{Bu}$-isocyanide. The formation of unique $\mathrm{Si}_{2} \mathrm{P}_{2}$ ring is observed when $\mathrm{LSi}^{\mathrm{I}}-\mathrm{Si}^{\mathrm{I}} \mathrm{L}$ is reacted with white phosphorus. All products were characterized by single crystal X-ray diffraction studies and multinuclear NMR spectroscopy. 


\title{
4.1 Introduction
}

\section{On s'engage et puis on voit}

\section{(First start and then you will see)}

\begin{abstract}
Napoleon
Probing and pushing the limits of metal-to-metal bonding has continuously been a strong motivation for the explorative synthetic chemist. Quite recent and spectacular reports such as the chromium-chromium quintuple bond ${ }^{30}$ and the zinc-zinc single bond ${ }^{29 b}$ in organometallic chemistry show that there is still plenty of room for new discoveries. A key to success over the last three decades has been the development of tailored steric shielding together with fine-tuning the electronic properties of the ancillary organic substituents at low coordinate metal centers across the periodic table. We had a recent success in isolating a new type of alkine analogue of silicon (35). It contains a Si-Si single bond with a lone pair of electrons on each silicon atom. Thus it can be considered as a bis-silylene or inter-connected silylene. So it is of interest to study the reactivity to ascertain the hallmark of such special class of compound. The stereo active lone pair of electrons on each silicon atom indicates increased reactivity. ${ }^{80-83}$ Now it is frequently experienced that the utilization of compounds with low valent silicon atom is always an alternative strategy to prepare silicon containing ring compounds. $^{84} \mathbf{3 5}$ is a new entry to compounds with low valent low coordinate silicon compound and may serve as a building block in organosilicon chemistry. Although the yield of $\mathbf{3 5}$ is only around 5\%, which can be a huge obstacle for pursuing further investigation, we remembered Napoleon Bonaparte and started our journey to make a systematic study of the reactivity of $\mathbf{3 5}$ towards unsaturated compounds. This would be of great interest to organic and organometallic chemists.
\end{abstract}




\subsection{Reactions of bis silylene with unsaturated organic compounds}

To probe the nature of the $\mathrm{Si}-\mathrm{Si}$ single bond we performed the reaction of $\mathbf{3 5}$ with benzophenone, benzil, $\mathrm{N}_{2} \mathrm{O}$, isocyanate, and white phophorus. Except in case of the reaction with benzil, the cleavage of the Si-Si bond was observed.

\subsubsection{Reaction with ketone}

$>\mathrm{C}=\mathrm{O}$ is the most versatile functional group in organic chemistry. It must be noted that despite the recent advancement in low valent silicon chemistry, the room temperature stable silanone $\left(\mathrm{R}_{2} \mathrm{Si}=\mathrm{O}\right)$, silicon analogue of ketone (Kipping's dream) is still elusive. The question arises whether it is possible to obtain a $[1+2]$ cycloaddition product or a cyclodisiloxane derivative from the reaction of $\mathbf{3 5}$ with benzophenone.

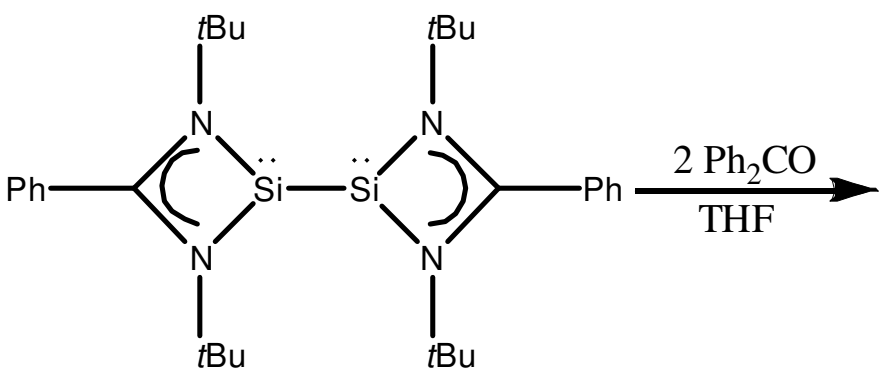

35

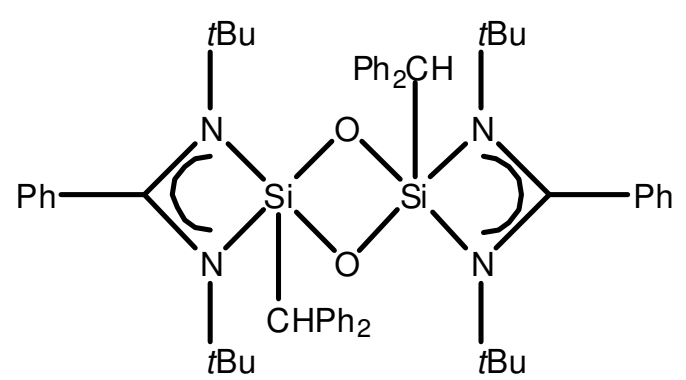

36

Scheme 4.1. Synthesis of $\mathbf{3 6}$

35 was treated with benzophenone in THF at ambient temperature under stirring overnight. After removal of the solvent under vacuum, $n$-hexane was added and the reaction mixture was stirred for $12 \mathrm{~h}$. After that the solution was concentrated and kept for crystallisation, which affords colorless crystals of $\mathbf{3 6}$ suitable for X-ray crystallography. The structure of $\mathbf{3 6}$ was confirmed by NMR spectroscopy, EI-MS spectrometry, and elemental analysis. The ${ }^{1} \mathrm{H}$ NMR spectrum exhibits a resonance $(\delta 1.27 \mathrm{ppm})$ which corresponds to the $t \mathrm{Bu}$ protons. A resonance at $\delta \quad 4.1 \mathrm{ppm}$ indicates the two $\mathrm{CH}$ protons. The ${ }^{29} \mathrm{Si}$ NMR spectrum shows a 
resonance at $\delta-86.25 \mathrm{ppm}$, which is comparable with that of the reported five-coordinate silicon $^{43}$ and differs distinctively from West's four- coordinate cyclodisiloxane $(\delta-22.02$ ppm). ${ }^{53 \mathrm{c}}$ The molecular ion is observed with the highest relative intensity in the EI-MS spectrum at $\mathrm{m} / \mathrm{z}$ 884. Compound 36 is well soluble in diethyl ether, THF, toluene, and partially soluble in $n$-hexane. It is stable in the solid state and in solution at room temperature under an inert atmosphere.

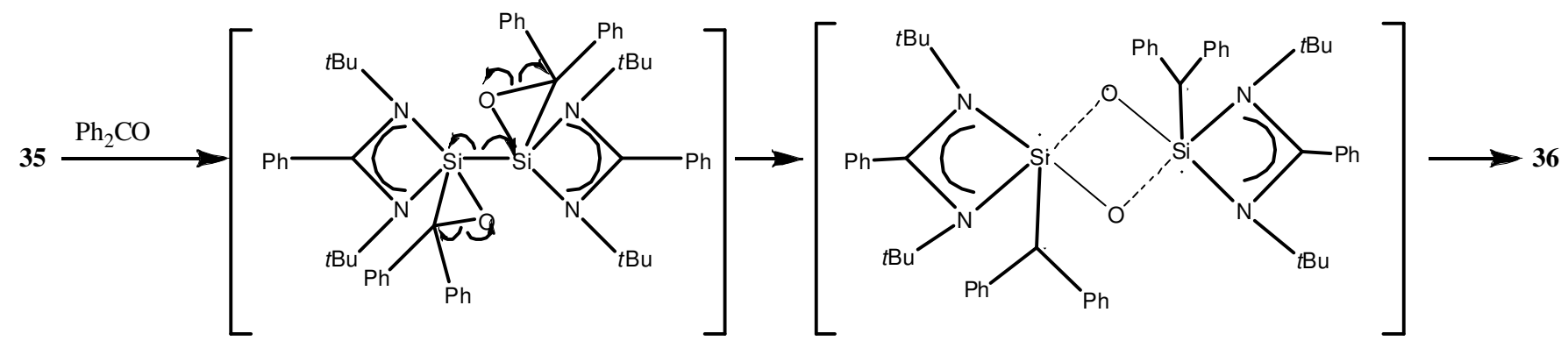

Scheme 4.2. Suggested mechanism for the formation of $\mathbf{3 6}$

The mechanism for the formation of $\mathbf{3 6}$ is not clear at this moment, and intermediates could not be observed. We propose that a [2+1] cycloaddition occurs as an initial step to give the respective three-membered $\mathrm{SiCO}$ cycles which subsequently rearrange to the final product under hydrogen abstraction from THF ${ }^{85}$ To prove the hydrogen abstraction from the solvent we performed a NMR scale reaction in THF- $d_{8}$, and we did not find any resonance for the $\mathrm{CH}$ proton. Furthermore a hydrogen abstraction from etheral solvent was also observed by Robinson et al. during the synthesis of diborane and diborene compounds. ${ }^{32 \mathrm{a}}$ The cleavage of the $\mathrm{Si}-\mathrm{Si}$ bond is not unexpected because So et al. showed recently that $\mathbf{3 5}$ is cleaved with $\mathrm{Br}_{2}$ under formation of a heteroleptic bromo silylene. ${ }^{86}$

The molecular structure of $\mathbf{3 6} \cdot 2$ toluene is shown in Figure $4.1 .^{44}$ Compound $\mathbf{3 6} \cdot 2$ toluene crystallizes in the monoclinic space group $P 2_{1} / n$. The structure consists of a rectangular 
cyclodisiloxane ring orthogonal to a slightly distorted planar skeleton containing the silicons and their pendant nitrogens. The two independent Si-O bond lengths are very close to each other (1.68(11) and 1.72(10) $⿱$ Á) and are slightly larger than the normal bond lengths found for other cyclic siloxanes. ${ }^{53}$ A twofold symmetry axis passes through the centroid of the siloxane ring, which is almost planar (sum of the internal angles $360.02^{\circ}$ ). The endocyclic Si-O-Si bond angle in the four-membered ring is $95.08^{\circ}$, which is very close to the $\mathrm{Si}-\mathrm{O}-\mathrm{Si}$ bond angle $\left(95.8^{\circ}\right)$ in $t \mathrm{Bu}_{4} \mathrm{Si}_{2} \mathrm{O}_{2}$ ring reported by West et al. ${ }^{53 \mathrm{c}}$ The amidinate ligands and $\mathrm{Ph}_{2} \mathrm{CH}$ groups are disposed above and below the $\mathrm{Si}_{2} \mathrm{O}_{2}$ ring in such a way that the Si centers exhibit trigonal bi-pyramidal coordination sites. ${ }^{87}$

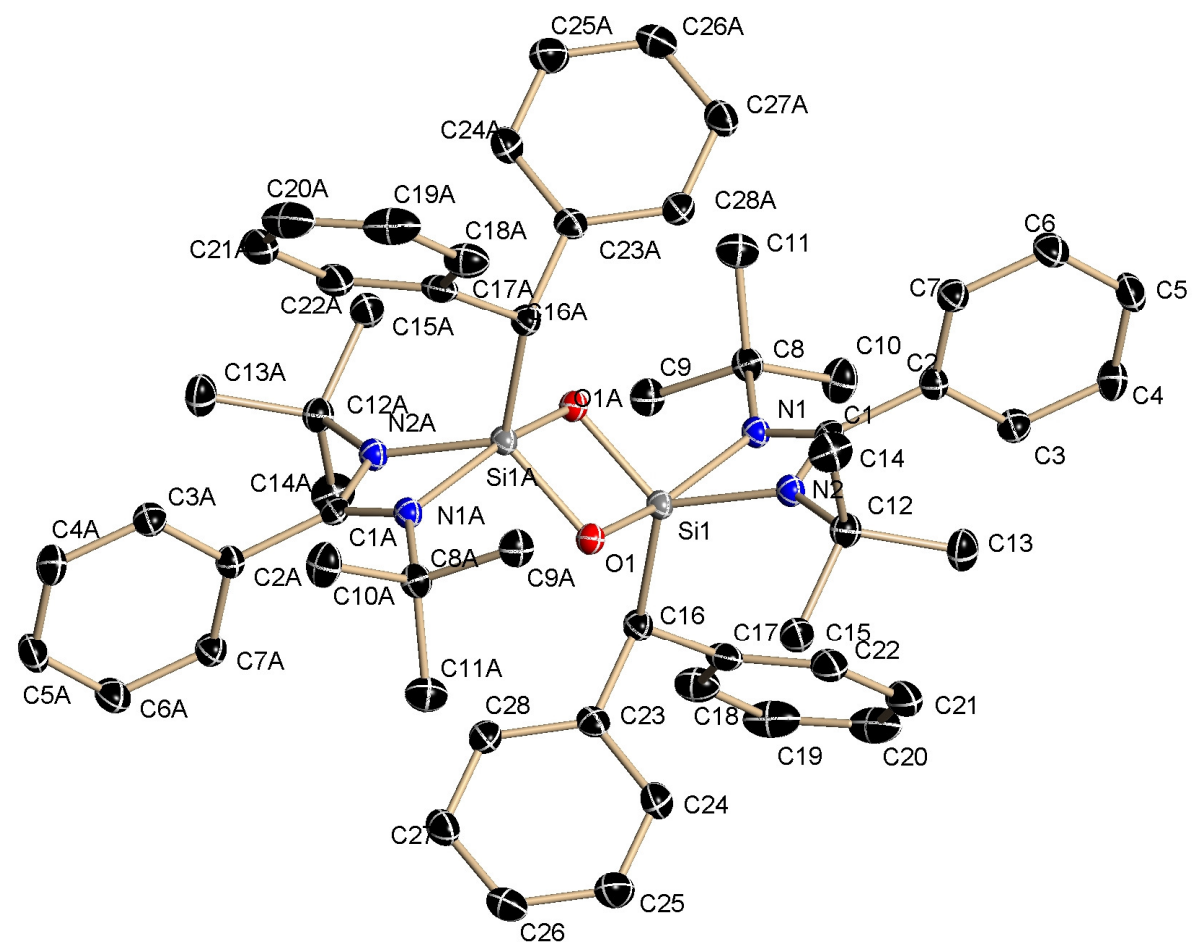

Figure 4.1. Crystal structure of $\mathbf{3 6} \cdot 2$ toluene. Hydrogen atoms, methyl groups at $C(8), C(12)$ and two toluene molecules are not shown for clarity. Anisotropic displacement parameters are depicted at the $50 \%$ probability level. Selected bond distances $(\AA)$ and bond angles (deg) $\mathrm{Si}(1)-\mathrm{O}(1 \mathrm{~A}) 1.68$ (11), $\mathrm{Si}(1)-\mathrm{O}(1)$ 1.72(10), $\mathrm{Si}(1)-\mathrm{N}(2)$ 1.83(13), $\mathrm{Si}(1)-\mathrm{C}(16)$ 1.93(15), $\mathrm{Si}(1)-\mathrm{N}(1) \quad 1.97(12), \quad \mathrm{O}(1)-\mathrm{Si}(1 \mathrm{~A}) \quad 1.68(11) ; \quad \mathrm{O}(1 \mathrm{~A})-\mathrm{Si}(1)-\mathrm{O}(1) \quad 84.93(5), \quad \mathrm{O}(1 \mathrm{~A})-\mathrm{Si}(1)-\mathrm{N}(2)$ 123.96(5), $\quad \mathrm{O}(1)-\mathrm{Si}(1)-\mathrm{N}(2) \quad$ 100.03(5), $\quad \mathrm{O}(1 \mathrm{~A})-\mathrm{Si}(1)-\mathrm{C}(16) \quad 117.07(6), \quad \mathrm{O}(1)-\mathrm{Si}(1)-\mathrm{C}(16)$ 101.77(6), N(2)-Si(1)-C(16) 116.28(6), O(1A)-Si(1)-N(1) 90.65(5), O(1)-Si(1)-N(1) 162.41(5), $\mathrm{N}(2)-\mathrm{Si}(1)-\mathrm{N}(1)$ 68.51(5), C(16)-Si(1)-N(1) 95.41(6), O(1A)-Si(1)-Si(1A) 43.07(3), O(1)-Si(1)$\mathrm{Si}(1 \mathrm{~A})$ 41.86(3), N(2)-Si(1)-Si(1A) 119.58(4). 
To explain the axial and equatorial arrangement we selected $\mathrm{Si}(1) . \mathrm{N}(2), \mathrm{O}(1 \mathrm{~A})$, and $\mathrm{O}(1)$, which reside in the equatorial positions, whereas $\mathrm{C}(16)$ and $\mathrm{N}(1)$ occupy the axial positions of the trigonal bipyramidal geometry. Another striking feature of this structure is the $\mathrm{Si}-\mathrm{Si}$ distance of 2.50(8) $\AA$ which is $6.38 \%$ longer than the normal Si-Si $\sigma$ bond distance (235 pm) and $3.73 \%$ longer compared to that in $\mathbf{3 5}(2.41 \AA \dot{A})$ and significantly longer than West's

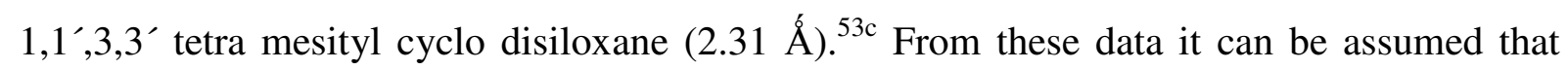
there is no bond between the two Si atoms. This assumption is also supported by MNDO calculations on the parent cyclodisiloxane, $\mathrm{H}_{4} \mathrm{Si}_{2} \mathrm{O}_{2},{ }^{53 \mathrm{~b}}$ which also provided no evidence for bonding between silicon atoms. In agreement with this, recent ab initio calculations on $\mathrm{H}_{4} \mathrm{Si}_{2} \mathrm{O}_{2}$ indicate that the cyclodisiloxane is best described as containing four equivalent localized $\mathrm{Si}-\mathrm{O}$ bonds with no appreciable $\sigma$ bonding between the silicon atoms. ${ }^{88}$ The calculated $\mathrm{Si}-\mathrm{O}-\mathrm{Si}$ bond angle for $\mathrm{H}_{4} \mathrm{Si}_{2} \mathrm{O}_{2}$ is $91.5^{\circ}$, similar to that found for $\mathbf{3 6}$, while the calculated Si-O bond length $(2.39 \AA)$ ) is reasonably shorter than those in 36. A possible qualitative explanation for the marked differences in bond length observed in $\mathbf{3 6}$ and West's $1,1^{\prime}, 3,3^{\prime}$ tetra mesityl cyclo disiloxane is as follows: The $3 p$ orbitals of $\boldsymbol{\pi}$ symmetry on silicon have low electron density compared to the $2 p$-orbitals on oxygen so that the diagonal antibonding interactions between oxygens are stronger than between the silicon atoms, leading to the diamond-shaped distortion found for West's disiloxane, ${ }^{53 \mathrm{c}}$ whereas in $\mathbf{3 6}$, due to the presence of four nitrogen atoms the electron density on silicon atoms is shifted towards the amidinato ligand causing longer Si-Si bond length. Here it is also worth to point out that recently Driess and co-workers reported the formation of cyclodisiloxane, where the two silicon atoms have different coordination numbers; one silicon atom is penta-, and the other one is tetra-coordinate, ${ }^{89}$ whereas in $\mathbf{3 6}$ both the Si centers are five -coordinate. 


\subsubsection{Reaction with diketone}

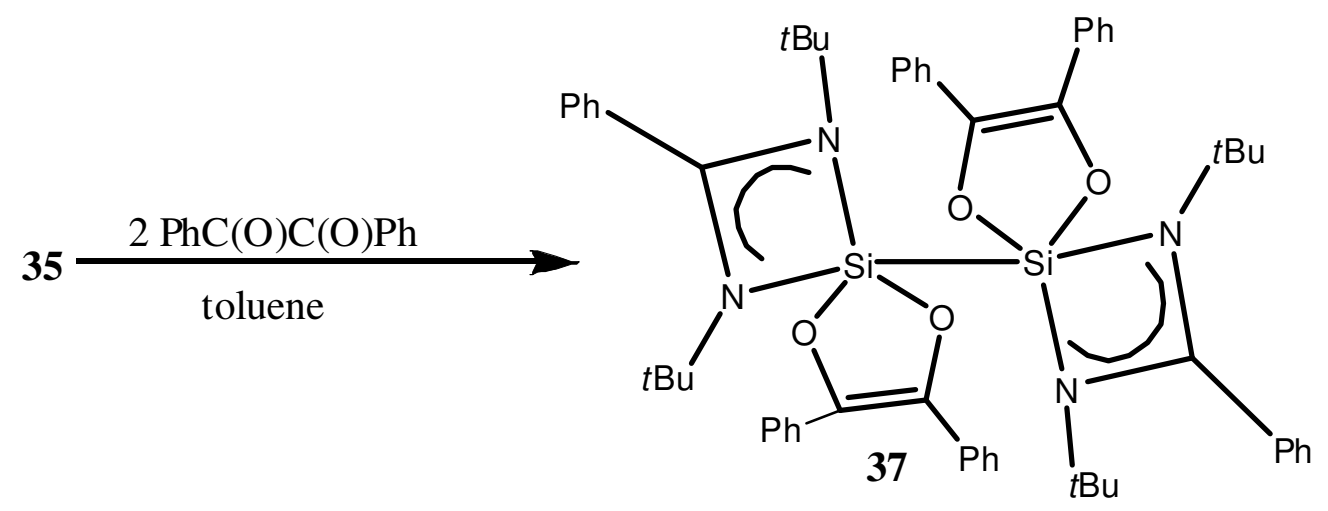

Scheme 4.3. Synthesis of 37

Compound $\mathbf{3 7}$ was formed by treatment of $\mathbf{3 5}$ with 2 equivalents of benzil in toluene overnight at room temperature (Scheme 4.3). After removal of the solvent under vacuum, $n$ hexane was added and the reaction mixture was stirred for $2 \mathrm{~h}$. After that the solution was concentrated and kept for crystallisation, which afforded colorless crystals of $\mathbf{3 7}$ suitable for X-ray crystallography. The structure of $\mathbf{3 7}$ was confirmed by NMR spectroscopy, EI-MS spectrometry, and elemental analysis. The ${ }^{29} \mathrm{Si}$ NMR spectrum shows a resonance at $\delta-79.75$ ppm that differs distinctively from that of $\mathbf{3 5}(\delta 76.15 \mathrm{ppm})$ and is comparable with that of the bis(silacyclopropene) derivative prepared by Ando et al. $(\delta-61.9 \mathrm{ppm}) .{ }^{43 a}$ Moreover it matches very well with those of the reported compounds with five-coordinate silicon. ${ }^{43}$ In the EI-MS spectrum the molecular ion was observed as the most abundant peak with highest relative intensity at $\mathrm{m} / \mathrm{z}$ 938.4. The most striking feature of the reaction is the preservation of the Si-Si bond after the reaction. 


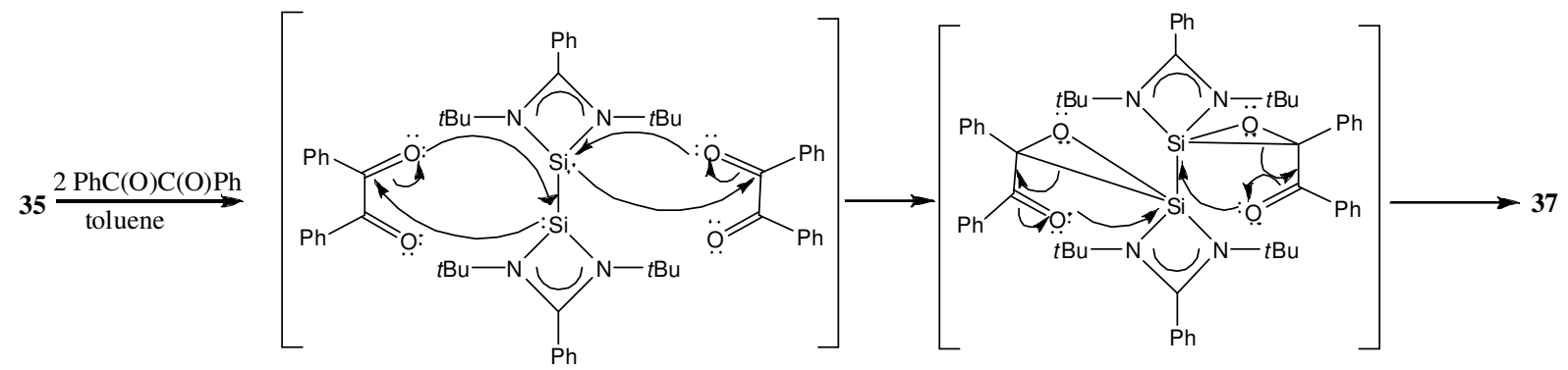

Scheme 4.4. Suggested mechanism for the formation of 37

The mechanism for the reaction seems to be obvious. $\mathbf{3 7}$ is formed unequivocally as a two times [1+4] cycloaddition product. Therefore the question raises why the reaction of $\mathbf{3 5}$ with diketone afforded a compound keeping the $\mathrm{Si}-\mathrm{Si}$ bond intact, whereas the similar reaction with ketone afforded a four-membered $\mathrm{Si}_{2} \mathrm{O}_{2}$ ring under the cleavage of the $\mathrm{Si}-\mathrm{Si}$ bond. To give the answer we decided to quote Basolo and Johnson from the book, "Coordination Chemistry", published in 1965. They stated: “In general, it has been observed that for ligands that do not contain double bonds, those that form five-membered metal-chelate rings give the most stable products. Chelate rings that contain either four atoms or more than six atoms have been observed, but they are relatively unstable and uncommon".90a The reason behind this observation is presumably the change in internal ring strain accompanying the change in coordination number of the ring atom participating in the reaction. For three-membered rings the internal strain arises primarily from the distortions of the normal bond angle whereas for five-membered rings the strain is attributed primarily to repulsion terms arising from unfavorable conformations. As a result five-membered rings are more stable compared to the three-membered ring compounds. $^{90 \mathrm{~b}}$ Therefore, the formation of the bis(silaepoxide) derivative is energetically not favourable although the situation is reverse in case of the bis(siladioxolene) derivative. Moreover, replacement of one $\mathrm{R}$ group $\left(\mathrm{R}=\mathrm{PhC}(\mathrm{N} t \mathrm{Bu})_{2} \mathrm{Si}\right)$ by 
one chlorine atom decreases the strain of the ring and favors the formation of monosila epoxide. ${ }^{9}$

The molecular structure of $\mathbf{3 7}$ was unequivocally established by a single crystal X-ray structure determination (Figure 4.2). ${ }^{44} 37$ crystallizes in the monoclinic space group $P 2{ }_{1} / c$ incorporating two disordered toluene molecules per unit cell on lattice positions. Important bond lengths and angles are given in the legend of Figure 4.2. The structure consists of two five-membered dioxolene rings connected by a $\mathrm{Si}-\mathrm{Si}$ bond. To avoid the steric congestion two dioxolene rings are situated in such a way that they are arranged trans to each other. Both the silicon atoms are penta-coordinate and adopt a distorted trigonal bipyramidal geometry. It is true for each of them that the axial positions are occupied by one oxygen atom and one nitrogen atom. The sums of angles between the equatorial atoms and the silicon atom are $354.45^{\circ}$ for $\mathrm{Si}(1)$ and 358.24 for $\mathrm{Si}(2)$. The axial substituents include an angle of $164.95^{\circ}$ with $\operatorname{Si}(1)$ and $167.67^{\circ}$ with $\mathrm{Si}(2)$. The distorted trigonal bipyramidal architecture induces different $\mathrm{Si}-\mathrm{N}$ and $\mathrm{Si}-\mathrm{O}$ distances for axial and equatorial substituents. This effect is very apparent for the $\mathrm{Si}-\mathrm{N}$ bonds in that the axial positions exhibit elongated silicon-nitrogen distances $(\mathrm{Si}(1)-$ $\left.\mathrm{N}_{\mathrm{ax}}: 1.994 \AA, \operatorname{Si}(2)-\mathrm{N}_{\mathrm{ax}}: 2.001 \AA\right)$ compared to the equatorial bond lengths $\left(\mathrm{Si}(1)-\mathrm{N}_{\mathrm{eq}}\right.$ : $\left.1.830 \AA, \mathrm{Si}(2)-\mathrm{N}_{\mathrm{ax}}: 1.816 \AA\right)$, but the fact is also observable at the $\mathrm{Si}-\mathrm{O}$ distances $(1.753 \AA$ and $1.744 \AA$ for the axial $\mathrm{Si}-\mathrm{O}$ distances; $1.698 \AA$ and $1.701 \AA$ for the equatorial $\mathrm{Si}-\mathrm{O}$ distances). The bond lengths appear well comparable with previously reported values. ${ }^{53}$ 


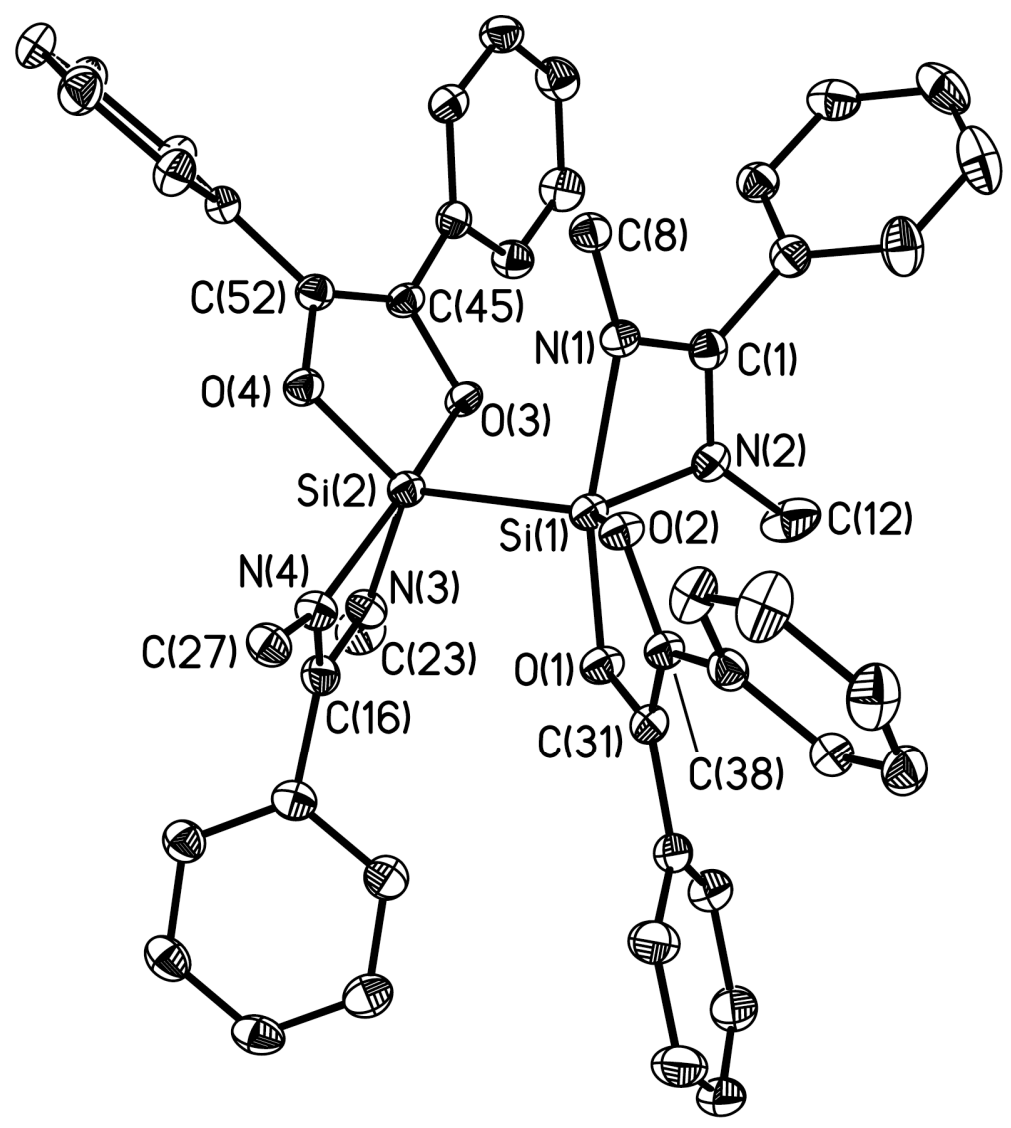

Figure 4.2. Molecular structure of 37. Hydrogen atoms as well as methyl groups and and the toluene lattice molecule are omitted for clarity. Selected bond distances $(\AA)$ and bond angles (deg) with standard deviations: $\mathrm{N}(1)-\mathrm{Si}(1)$ 1.9938(14), $\mathrm{N}(2)-\mathrm{Si}(1)$ 1.8304(15), N(3)-Si(2) 1.8163(14), N(4)-Si(2) 2.0008(14), O(1)-Si(1) 1.7531(12), O(2)-Si(1) 1.7008(12), O(3)-Si(2) 1.7443(12), O(4)-Si(2) 1.6984(12), Si(1)-Si(2) 2.3628(7); O(2)-Si(1)-O(1) 89.26(6), O(2)$\mathrm{Si}(1)-\mathrm{N}(2) \quad 114.81(6), \quad \mathrm{O}(1)-\mathrm{Si}(1)-\mathrm{N}(2) \quad 99.83(6), \quad \mathrm{O}(2)-\mathrm{Si}(1)-\mathrm{N}(1) \quad 87.50(6), \mathrm{O}(1)-\mathrm{Si}(1)-\mathrm{N}(1)$ 164.95(6), N(2)-Si(1)-N(1) 68.43(6), O(2)-Si(1)-Si(2) 128.85(5), O(1)-Si(1)-Si(2) 97.42(4), $\mathrm{N}(2)-\mathrm{Si}(1)-\mathrm{Si}(2)$ 113.79(5), N(1)-Si(1)-Si(2) 96.05(4), O(4)-Si(2)-O(3) 89.93(6), O(4)-Si(2)$\mathrm{N}(3) \quad 118.59(6), \quad \mathrm{O}(3)-\mathrm{Si}(2)-\mathrm{N}(3) \quad 100.46(6), \quad \mathrm{O}(4)-\mathrm{Si}(2)-\mathrm{N}(4) \quad 90.35(6), \quad \mathrm{O}(3)-\mathrm{Si}(2)-\mathrm{N}(4)$ 167.61(6), N(3)-Si(2)-N(4) 68.65(6), O(4)-Si(2)-Si(1) 124.90(5), O(3)-Si(2)-Si(1) 93.37(4), $\mathrm{N}(3)-\mathrm{Si}(2)-\mathrm{Si}(1)$ 114.75(5), N(4)-Si(2)-Si(1) 96.61(4).

The most noticeable feature in the structure is that the $\mathrm{Si}-\mathrm{Si}$ bond is not cleaved during the reaction, but the bond length is reduced to $2.36 \AA$ when compared to the $\mathrm{Si}-\mathrm{Si}$ bond length in the parent starting material $35(2.41 \AA)$. The new Si-Si bond distance is only about $0.02 \AA$ longer than the sum of the Si covalent radii $(2.34 \AA)^{76}$ and matches excellently with the $\mathrm{Si}-\mathrm{Si}$ single bond distance in $\alpha$-silicon $(2.36 \AA) .{ }^{77}$ The shortening of the Si-Si bond length is perhaps due to the nonexistence of the lone pair-lone pair repulsion in 37. Another important 
facet of the structure is the $\mathrm{C}-\mathrm{C}$ bond length in the dioxolene rings $(1.34 \AA$ and $1.35 \AA$ ), which are in good agreement with those of $\mathrm{C}-\mathrm{C}$ double bonds. ${ }^{91}$

\subsubsection{Reaction with Nitrous Oxide}

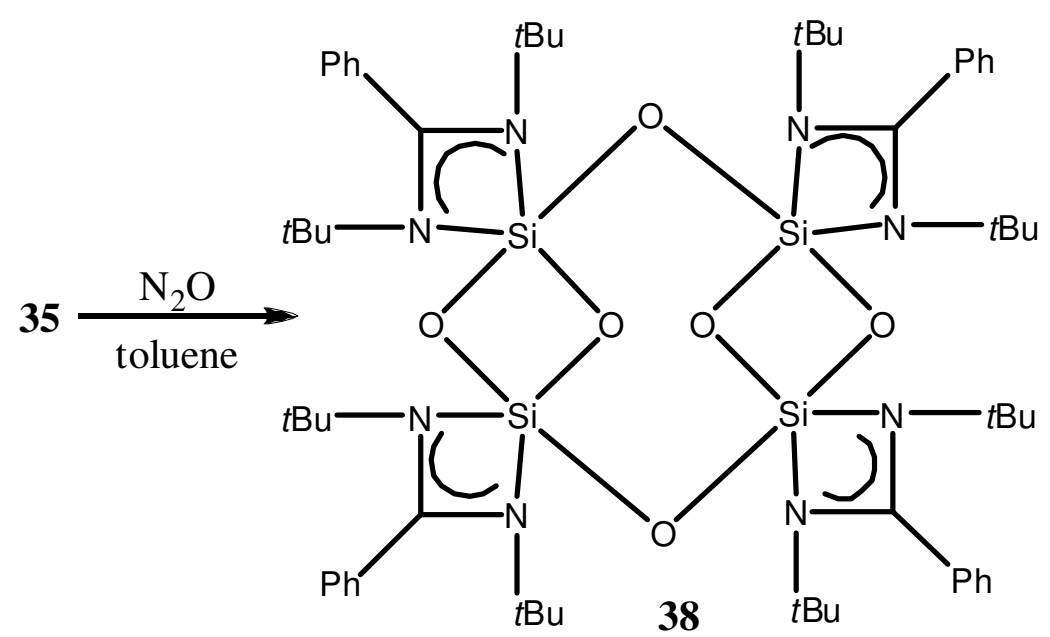

Scheme 4.5. Synthesis of $\mathbf{3 8}$

Exposure of a red solution of $\mathbf{3 5}$ in toluene to $\mathrm{N}_{2} \mathrm{O}$ at room temperature led to rapid decoloration and formation of a colorless compound. Recrystallization of the latter in a toluene/ $n$-hexane solution at room temperature furnished colorless crystals of $\mathbf{3 8}$ suitable for single crystal X-ray diffraction. To our surprise, $\mathbf{3 8}$ contains two four-membered disiloxane rings which are bridged by two oxygen atoms. This is a new class of compounds, which has not been reported so far. $\mathbf{3 8}$ was fully characterized by single crystal X-ray diffraction, NMR spectroscopy, EI-MS spectrometry, and elemental analysis. In the ${ }^{1} \mathrm{H}$ NMR spectrum two types of resonances were observed, one for $t \mathrm{Bu}$ protons and another for the phenyl rings. In the ${ }^{29} \mathrm{Si}$ NMR spectrum a sharp resonance was outlined $(\delta-111.02 \mathrm{ppm})$, which corresponds to the five-coordinate silicon atom ${ }^{43}$ and differs distinctly from that of $\mathbf{3 6}(\delta-86.25 \mathrm{ppm})$ and 
Driess' cyclodisiloxane $(\delta-60.7$ and $-119.2 \mathrm{ppm}) .{ }^{89}$ The molecular ion is observed with the highest relative intensity in the EI-MS spectrum at $\mathrm{m} / \mathrm{z}$ 1132. All the silicon atoms in $\mathbf{3 8}$ are five-coordinate.

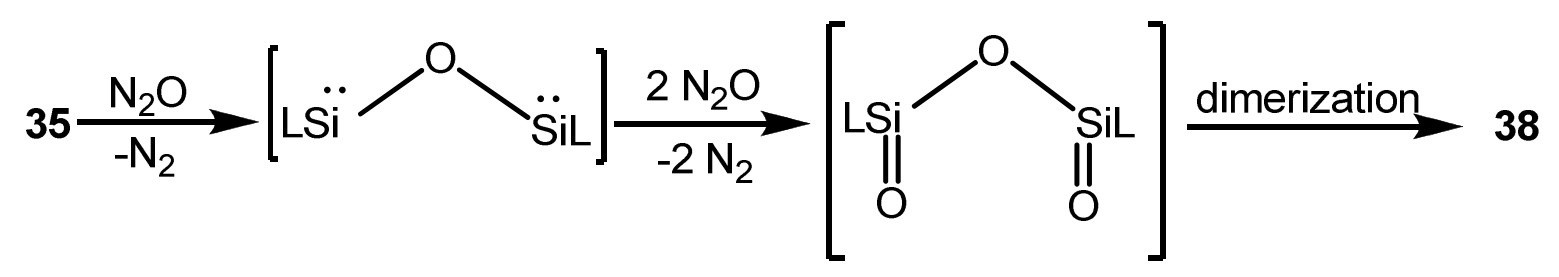

Scheme 4.6. Suggested mechanism for the formation of $\mathbf{3 8}$

Like 36, the mechanism for the formation of $\mathbf{3 8}$ is unknown, but it can be suggested that the $\mathrm{Si}-\mathrm{Si}$ bond is cleaved under insertion of an oxygen atom from $\mathrm{N}_{2} \mathrm{O}$. Then each of the lone pair of electrons at silicon reacts with $\mathrm{N}_{2} \mathrm{O}$ to give the respective $\mathrm{Si}=\mathrm{O}$ which in situ dimerizes to afford 38 due to the highly polar nature of $\mathrm{Si}^{\delta+}=\mathrm{O}^{\delta-} \cdot 5 \mathrm{~h}$

The molecular structure of $\mathbf{3 8}$ toluene was unequivocally established by single crystal X-ray diffraction (Figure 4.3). ${ }^{44} \mathbf{3 8}$ toluene crystallizes in the monoclinic space group $P 2_{1} / n$. Selected bond lengths and bond angles are given in the legend of Figure 4.3. 


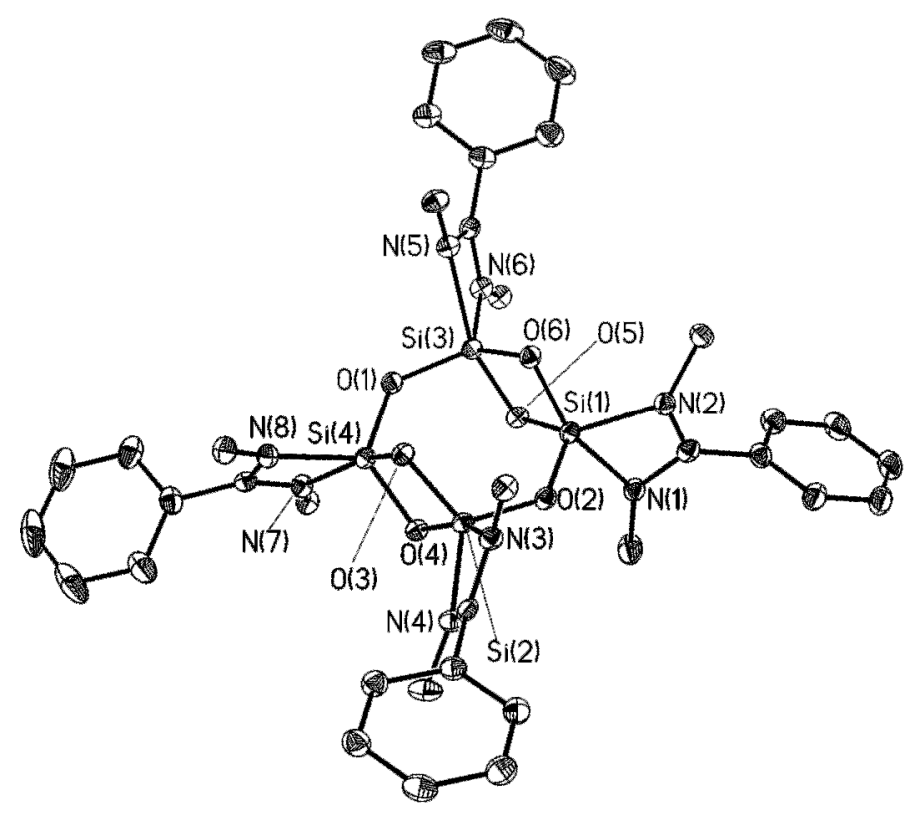

Figure 4.3. Crystal structure of 38- toluene. Hydrogen atoms as well as methyl groups and the toluene molecule are omitted for clarity. Selected bond distances $(\AA)$ and bond angles (deg) with standard deviations: $\mathrm{O}(1)-\mathrm{Si}(3)$ 1.64(13), O(1)-Si(4) 1.65(13), O(2)-Si(1) 1.64(13), $\mathrm{O}(2)-\mathrm{Si}(2)$ 1.64(13), O(3)-Si(2) 1.66(13), O(3)-Si(4) 1.73(13), O(4)-Si(4) 1.65(13), O(4)-Si(2) 1.72(13), O(5)-Si(1) 1.66(13), O(5)-Si(3) 1.72(13), O(6)-Si(3) 1.66(13), O(6)-Si(1) 1.72(13), $\mathrm{N}(1)-\mathrm{Si}(1)$ 1.97(16), N(2)-Si(1) 1.85(16), N(3)-Si(2) 1.98(16), N(4)-Si(2) 1.84(16), N(5)-Si(3) 2.02(16), $\quad \mathrm{N}(6)-\mathrm{Si}(3) \quad 1.84(16), \quad \mathrm{N}(7)-\mathrm{Si}(4)$ 1.99(16), $\mathrm{N}(8)-\mathrm{Si}(4) \quad 1.85(16) ; \quad \mathrm{Si}(3)-\mathrm{O}(1)-\mathrm{Si}(4)$ 129.96(8), $\mathrm{Si}(1)-\mathrm{O}(2)-\mathrm{Si}(2)$ 130.94(8), $\mathrm{Si}(2)-\mathrm{O}(3)-\mathrm{Si}(4)$ 94.28(6), $\mathrm{Si}(4)-\mathrm{O}(4)-\mathrm{Si}(2)$ 94.70(6), $\mathrm{Si}(1)-\mathrm{O}(5)-\mathrm{Si}(3)$ 94.28(6), $\mathrm{Si}(3)-\mathrm{O}(6)-\mathrm{Si}(1)$ 94.26(6), O(2)-Si(1)-O(5) 117.48(7), O(2)-Si(1)$\mathrm{O}(6) \quad$ 102.92(6), $\mathrm{O}(5)-\mathrm{Si}(1)-\mathrm{O}(6) \quad 85.25(6), \quad \mathrm{O}(2)-\mathrm{Si}(1)-\mathrm{N}(2) \quad 113.48(7), \quad \mathrm{O}(5)-\mathrm{Si}(1)-\mathrm{N}(2)$ 127.11(7), O(6)-Si(1)-N(2) 96.67(7), O(2)-Si(1)-N(1) 96.71(7), O(5)-Si(1)-N(1) 92.40(7), O(6)$\mathrm{Si}(1)-\mathrm{N}(1)$ 158.96(7), N(2)-Si(1)-N(1) 68.21(7), O(2)-Si(2)-O(3) 117.04(7), O(2)-Si(2)-O(4) 101.85(6), O(3)-Si(2)-O(4) 85.15(6), O(2)-Si(2)-N(4) 116.65(7), O(3)-Si(2)-N(4) 124.52(7), $\mathrm{O}(4)-\mathrm{Si}(2)-\mathrm{N}(4)$ 96.82(7), O(2)-Si(2)-N(3) 96.58(6).

The most important feature of $\mathbf{3 8}$. toluene is that two four-membered rings are connected by two oxygen atoms. The ring actually consists of two four-membered disiloxane rings which are parallel to each other and connected by a bridging oxygen atom. The amidinato ligands are each arranged orthogonally to the four-membered disiloxane ring. All the silicon atoms are five-coordinate and exhibit a trigonal bipyramidal geometry. Three sites of each silicon atom are occupied by the three oxygen atoms whereas the nitrogen atoms from the amidinato ligand occupy the remaining two coordination sites. To explain the axial and equatorial arrangement of the silicon atoms, we chose one silicon atom in the ring designated as $\mathrm{Si}(4)$. 
From the bond lengths and angles data it is revealed that $\mathrm{N}(7)$ and $\mathrm{O}(3)$ reside in the axial positions and $\mathrm{O}(1), \mathrm{O}(4)$, and $\mathrm{N}(8)$ occupy the equatorial positions. The sum of angles between the equatorial atoms and $\mathrm{Si}(4)$ is $357.9^{\circ}$ and the two axial atoms include an angle of $159.5^{\circ}$ with $\mathrm{Si}(4)$. The arrangement holds truth for all the silicon atoms in the ring where one oxygen atom of the disiloxane moiety and one nitrogen atom from the amidinato ligand occupy axial positions and two oxygen atoms and another nitrogen atom from the ligand occupy equatorial positions. It is noticeable that the $\mathrm{Si}-\mathrm{N}$ as well as the $\mathrm{Si}-\mathrm{O}$ bond lengths differ significantly depending on whether equatorial or axial positions are occupied. The Si-N distances range from $1.97 \AA$ to $2.02 \AA$ for the axial nitrogen atoms while the equatorial Si-N bond lengths are between $1.84 \AA$ and $1.85 \AA$ and thus significantly shorter. The Si-O bond lengths in the disiloxane rings exhibit a similar behaviour with axial Si-O bond lengths ranging from $1.72 \AA$ to $1.73 \AA$ and shorter equatorial Si-O bond lengths ranging from $1.65 \AA$ to $1.66 \AA$. All these $\mathrm{Si}-\mathrm{O}$ bond lengths are quite similar with those found for $\mathbf{3 6}, \mathbf{3 7}$, and $\mathrm{HSi}\left(\mathrm{L}^{\prime}\right)-\mathrm{O}-(\mathrm{L}) \mathrm{Si}^{89}$

\subsubsection{Reaction with tert-butyl isocyanate}

$$
35 \underset{\text { toluene }}{\stackrel{t \mathrm{BuN}=\mathrm{C}=\mathrm{O}}{\longrightarrow}} 38+t \mathrm{Bu}-\mathrm{N} \equiv \mathrm{C}
$$

Scheme 4.7. Alternative synthesis of $\mathbf{3 8}$

Treatment of $\mathbf{3 5}$ with $t \mathrm{Bu}$-isocyanate led to the formation of $\mathbf{3 8}$. The composition and constitution was characterized by NMR spectroscopy, EI-MS spectrometry, and elemental analysis and compared with the previously reported data. The formation of $t \mathrm{Bu}$-isocyanide was observed in the ${ }^{1} \mathrm{H}$ NMR spectrum. 


\subsubsection{Reaction with white phosphorus}

With the advent of "Isolobal Principle"92 and its application in organometallic and main group chemistry, a considerable attention was paid to the chemistry of phosphorus because the $\mathrm{CH}$ fragment in acyclic and cyclic hydrocarbon can be formally replaced by a naked $\mathrm{P}$ atom. Scherer and his coworkers took the initiative and reported a myriad of polycyclic phosphorus compounds using the $\mathrm{P} \leftrightarrow \mathrm{CH}$ isolobal relationship. ${ }^{93}$ The analogy between hydrocarbon species and organophosphorus compounds has been described in the book, "Phosphorus: The Carbon Copy". ${ }^{94}$ Following this, several review articles appeared addressing the activation of white phosphorus mediated by early transition metals, ${ }^{95}$ late transition metals, ${ }^{96}$ and main group elements. ${ }^{97}$ Nevertheless the activation of phosphorus by low-valent group 14 elements is still in its infancy. Driess and coworkers documented the synthesis of $\mathrm{SiP}_{4}$ and $\mathrm{Si}_{2} \mathrm{P}_{4}$ cage compounds by the reaction between silylene and white phosphorus. ${ }^{65 n}$ After that, the same group illustrated the formation of heterobinuclear tetra phosphorus complexes with a $\operatorname{Si}(\mu$, $\left.\eta^{2: 2}-\mathrm{P}_{4}\right) \mathrm{Ni}$ core from the reaction between $\mathrm{SiP}_{4}$ cage compound and $\beta$-diketiminato nickel(I) complex. ${ }^{98}$ These results further show that there is plenty of room for new discovery of novel interesting compounds containing silicon and phosphorus atoms. In view of these we were interested to investigate the reaction of $\mathbf{3 5}$ and white phosphorus.

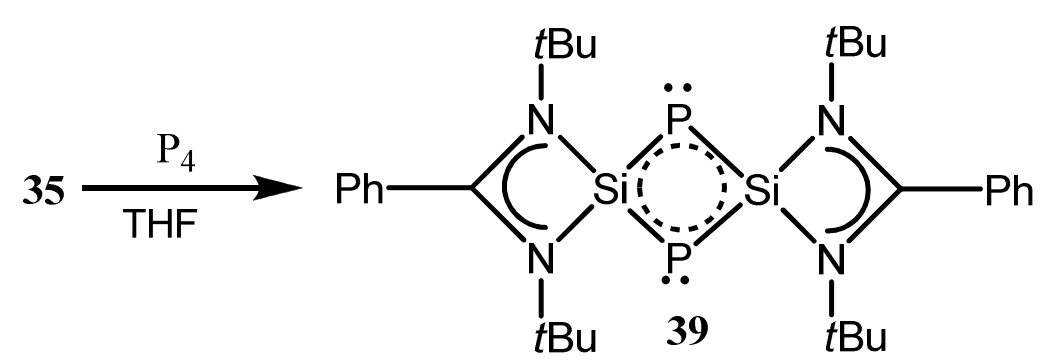

Scheme 4.8. Synthesis of 39 
Treatment of equimolar amounts of $\mathbf{3 5}$ and white $\mathrm{P}_{4}$ in THF overnight resulted in the formation of the $\mathrm{Si}_{2} \mathrm{P}_{2}$ unit stabilized by the amidinato ligand $\left(\mathrm{L}_{2} \mathrm{Si}_{2} \mathrm{P}_{2} ; \mathrm{L}=\mathrm{PhC}(\mathrm{N} t \mathrm{Bu})_{2}\right)(39)$. After recrystallisation from THF, 39 was isolated as air- and moisture-sensitive yellow crystals with $60 \%$ yield. The ${ }^{1} \mathrm{H}$ NMR spectrum shows one new singlet at $\delta 1.36 \mathrm{ppm}$, which is deshielded in comparison to that of $\mathbf{3 5}(\delta 1.23 \mathrm{ppm})$. The ${ }^{31} \mathrm{P}$ NMR spectrum of $\mathbf{3 9}$ displays a singlet with silicon satellite at $\delta-166 \mathrm{ppm}\left(J_{\mathrm{Si}-\mathrm{P}}=75 \mathrm{~Hz}\right)$. The ${ }^{29} \mathrm{Si}$ NMR spectrum of 39 shows a resonance at $\delta 25.6 \mathrm{ppm}$.

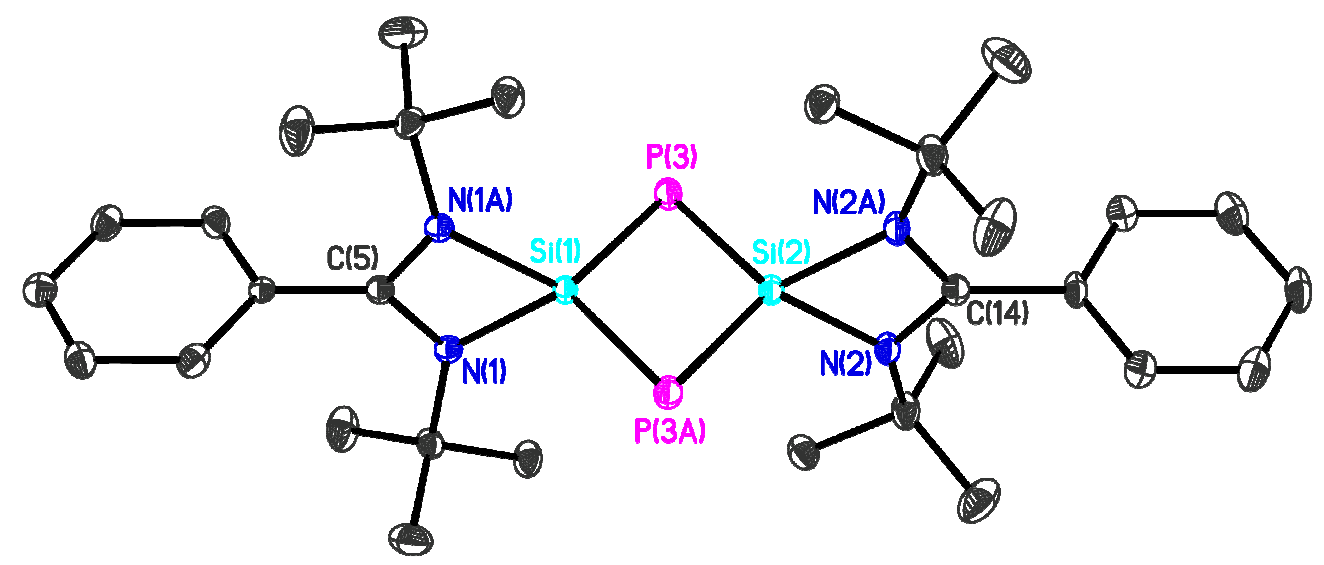

Figure 4.4. Molecular structure of compound 39. Anisotropic displacement parameters are depicted at $50 \%$ probability level. Hydrogen atoms have been removed for clarity. Selected bond distances $(\AA)$ and bond angles (deg) $\mathrm{N}(1)-\mathrm{Si}(1)$ 1.8585(14), N(2)-Si(2) 1.8524(15), $\mathrm{P}(3)-\mathrm{Si}(2) \quad 2.1737(6), \quad \mathrm{P}(3)-\mathrm{Si}(1) \quad 2.1742(6), \quad \mathrm{Si}(1)-\mathrm{N}(1) \# 1 \quad$ 1.8586(14), $\quad \mathrm{Si}(1)-\mathrm{P}(3) \# 1$ 2.1742(6), Si(1)-Si(2) 2.5693(9), Si(2)-N(2)\#1 1.8524(15), Si(2)-P(3)\#1 2.1737(6); C(14)$\mathrm{N}(2)-\mathrm{Si}(2)$ 91.41(11), C(10)-N(2)-Si(2) 137.42(11), Si(2)-P(3)-Si(1) 72.45(2), N(1)-Si(1)-N(1)\# 170.53(9), $\quad \mathrm{N}(1)-\mathrm{Si}(1)-\mathrm{P}(3) \quad 118.61(4), \quad \mathrm{N}(1) \# 1-\mathrm{Si}(1)-\mathrm{P}(3) 119.11(4), \quad \mathrm{N}(1)-\mathrm{Si}(1)-\mathrm{P}(3) \# 1$ 119.11(4), N(1)\#1-Si(1)-P(3)\#1 118.61(4), P(3)-Si(1)-P(3)\#1 107.54(3), $\quad P(3)-S i(2)-$ $\mathrm{P}(3) \# 1$ 107.57(3)

The proposed constitution of $\mathbf{3 9}$ was confirmed by single crystal X-ray diffraction (Figure 4.4). Crystals of 39 were grown by cooling a concentrated THF solution to $-32{ }^{\circ} \mathrm{C}$; the complex crystallises in the monoclicnic space group $C 2 / c$ with a mirror plane bisecting the molecule. ${ }^{[19]}$ Importatnt bond lengths and angles are provided in the legend of Figure 4.4. The most apparent feature of the structure is the four-membered $\mathrm{Si}_{2} \mathrm{P}_{2}$ ring. The planar ring consists of four equivalent silicon -phosphorus bonds of 2.17(6) $\AA$. The value is the shorter 
than of the Si-P single bond and longer than that of double bond and matches well with that of 32. It indicates that all $\mathrm{Si}-\mathrm{P}$ bonds are in resonance. It is further supported from the $\mathrm{Si} \cdots \mathrm{Si}$ separation $(2.57 \AA)$, which suggests that there is no Si-Si bond in 39. Like 32, the silicon atoms in $\mathbf{3 9}$ exhibit a distorted tetrahedral geometry, whereas the phosphorus adopts trigonal planar geometry. Another important feature are the two equivalent Si-P-Si bond angles of $72.45(2)^{\circ}$. The value is slightly increased compared to that of 32. The two P-Si-P bond angles are also exactly the same $\left(107.5(3)^{\circ}\right)$. All these data indicate the extensive delocalization of the $4 \pi$ electrons over a four -center bonding molecular orbital comprised of the $\mathrm{p}_{\mathrm{z}}$-atomic orbital of silicon and phosphorus.

\subsection{Conclusion}

We have prepared a four-membered $\mathrm{Si}_{2} \mathrm{O}_{2}$ ring with five-coordinate silicon atoms. Compound 36 is formed by oxidative addition of benzophenone under cleavage of the ketone bond and hydrogen abstraction from THF. We reacted 35 with $\mathrm{N}_{2} \mathrm{O}$ to afford compound $\mathbf{3 8}$ with two four-membered rings bridged by two oxygen atoms. Surprisingly no cleavage of the Si-Si bond was observed when $\mathbf{3 5}$ was treated with benzil. The reaction of $\mathbf{3 5}$ with white phosphorus led to the formation of a unique cyclobutadiene analogue without a carbon atom. The composition and constitution of $\mathbf{3 6}, \mathbf{3 7}, \mathbf{3 8}$, and $\mathbf{3 9}$ has been supported by NMR spectroscopy, EI-MS spectrometry, and by single crystal X-ray diffraction. 


\section{Chapter 5}

\section{Synthesis, Structure, and Theoretical Investigation}

\section{of Amidinato Supported 1,4-disilabenzene}

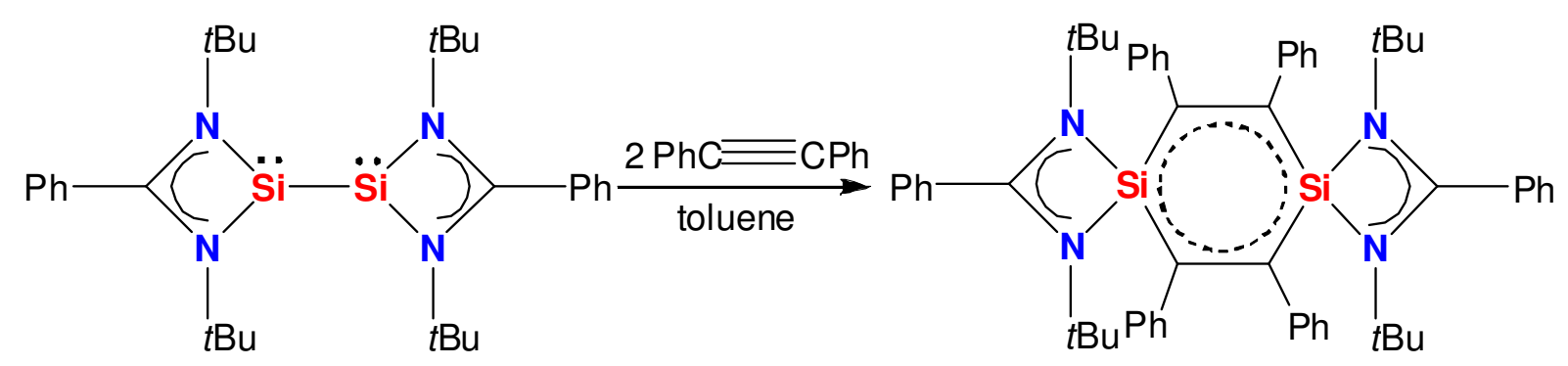

\footnotetext{
Abstract

Reaction of LSi-SiL (35) $\left(\mathrm{L}=\mathrm{PhC}(\mathrm{N} t \mathrm{Bu})_{2}\right)$ with biphenyl alkine gave the first example of a room temperature stable 1,4-disilabenzene. The compound was characterized by single crystal X-ray diffraction studies. NICS calculations showed that the compound has some aromatic character.
} 


\subsection{Introduction}

I was sitting writing at my textbook but the work did not progress; my thoughts were elsewhere. I turned my chair to the fire and dozed. Again the atoms were gambolling before my eyes. This time the smaller groups kept modestly in the background. My mental eye, rendered more acute by the repeated visions of the kind, could now distinguish larger structures of manifold confirmation: long rows, sometimes more closely fitted together all twining and twisting in snake like motion. But look! What was that? One of the snakes had seized hold of its own tail, and the form whirled mockingly before my eyes. As if by a flash of lightning I awoke; and this time also I spent the rest of the night in working out the rest of the hypothesis. Let us learn to dream, gentlemen, then perhaps we shall find the truth... But let us beware of publishing our dreams till they have been tested by waking understanding.

\section{Kekulé}

This is the story behind the discovery of the structure of benzene, perhaps the most important discovery in chemistry so far. It paves the way for aromatic compounds, which are wonderful edifices in organic and organometallic chemistry. Although the concept of aromaticity has almost exclusively emerged from the domain of organic chemistry, many other heteroatomic examples have been identified ever since. ${ }^{99}$ Borazine, popularly known as "inorganic benzene" is believed to be aromatic because it is isostructural to benzene, and they share some similar physical properties. ${ }^{100 a, 100 b}$ In recent years numerous anionic boron compounds, which are found to be aromatic, have been isolated by other research groups. ${ }^{100 c-100 f}$ Owing to the similarities between carbon and its closest congener silicon it was also expected that siliconsubstituted benzene would show aromatic character and has become a synthetic and theoretical attraction for many research groups. The aromatic nature of silicon-substituted benzene has been predicted theoretically, ${ }^{101}$ and some properties were observed spectroscopically in the gas phase and in low temperature matrices ${ }^{102}$ but due to the high 
reactivity the synthesis of silabenzene was not accomplished. Märkl and Schlosser came to the closest and reported the generation and NMR observation of the 1,4-di-tert-butyl-2,6bis(trimethylsilyl)-1-silabenzene. ${ }^{103}$ However, the major breakthrough was achieved when Tokitoh and coworkers synthesized stable silabenzene by introducing the sterically encumbered 2,4,6-tris[bis(trimethylsilyl)-methyl]phenyl substituent at the silicon atom. ${ }^{104}$ Parallel to this, Ando et al. reported the synthesis of 1,4-disila-(Dewar-benzene). ${ }^{105}$ Following this Sekiguchi and coworkers accounted the synthesis of 1,2-disilabenzene by treating $\mathrm{RSi} \equiv \mathrm{SiR}\left(\mathrm{R}=\mathrm{Si}\left(\mathrm{CH}(\mathrm{TMS})_{2} i \mathrm{Pr}\right)\right.$ with $\mathrm{PhC} \equiv \mathrm{CH}$ which underwent a $[2+4]$-cycloaddition. ${ }^{60}$ But the most outstanding result so far came very recently with the isolation of a tricyclic aromatic isomer of hexasilabenzene by Scheschkewitz et al. ${ }^{106}$ All these molecules are notable additions to the diverse progress of organosilicon chemistry.

In contrast, 1,4-disilabenzene has never been isolated as stable compound, although there are some reports of the direct observation by UV-Vis spectroscopy of the parent 1,4disilabenzene in a frozen Ar matrix at $10 \mathrm{~K}^{107}$ and of the chemical trapping of intermediate 1,4-disilabenzenes with $\mathrm{MeOH}$ or alkine derivatives. ${ }^{108}$ Therefore, in spite of all these remarkable results, it would not be an overstatement that the synthesis and isolation of 1,4disilabenzene still remains a synthetic challenge and there is still plenty of room for new discoveries. ${ }^{109}$ A key to success behind these results has been the development of tailor made steric shielding together with fine tuning the electronic properties of the ancillary organic ligands at low valent silicon atoms. Recently, we outlined the successful isolation of $\left[\mathrm{PhC}(\mathrm{N} t \mathrm{Bu})_{2}\right]_{2} \mathrm{Si}_{2}(\mathbf{3 5})$ stabilized by bulky amidinato ligands. 35 consists of a $\mathrm{Si}-\mathrm{Si}$ single bond and a lone pair of electrons at each Si atom. The formal oxidation state of each silicon atom in $\mathbf{3 5}$ is $+\mathrm{I}$ or, according to the suggestion of Frenking et al., compound $\mathbf{3 5}$ contains three-valent silicon atoms of formal oxidation state $+\mathrm{I} .{ }^{110}$ Reviewing the literature results we 
expected that the reaction of alkine with $\mathbf{3 5}$ may afford the formation of elusive 1,4disilabenzene or a diradical of 1,4-disilacyclohexadiene. Herein, we report the synthesis, structural analysis, and theoretical investigation of a 1,4-disilabenzene supported by bulky amidinato ligands with $t \mathrm{Bu}$ substituents on the nitrogen atoms. The compound is definitely stable at room temperature both in solution and in the solid state.

\subsection{Result and discussion}

We found that the reaction of $\mathbf{3 5}$ with biphenyl alkine in toluene at room temperature in a 1:2 molar ratio resulted in the formation of $\operatorname{LSi}\left(\mathrm{C}_{2} \mathrm{Ph}_{2}\right)\left(\mathrm{C}_{2} \mathrm{Ph}_{2}\right) \operatorname{SiL}\left(\mathrm{L}=\mathrm{PhC}(\mathrm{N} t \mathrm{Bu})_{2}\right)(\mathbf{4 0})$ with the cleavage of the central Si-Si bond of $\mathbf{3 5}$ (Scheme 5.1). $\mathbf{4 0}$ was isolated as air sensitive dark red crystals in $30 \%$ yield by recrystallization of the reaction mixture in toluene. $\mathbf{4 0}$ was characterized by X-ray crystallography, NMR spectroscopy, EI-MS spectrometry, and moreover we performed DFT calculations to elucidate the nature of the bonding. In the ${ }^{29} \mathrm{Si}$ NMR a resonance was exhibited at $\delta-18.05 \mathrm{ppm}$. The most striking result of the reaction is the six-membered ring, which is nearly planar and contains $6 \pi$-electrons. Because of the topological similarities to the singlet diradical of boron reported by Bertrand et al., we initially did not eliminate the possibility of the formation of a silicon diradical, ${ }^{111}$ but the EPR experiments even at elevated temperature did not support the hypothetical 1,4disilacyclohexadiene diradical.
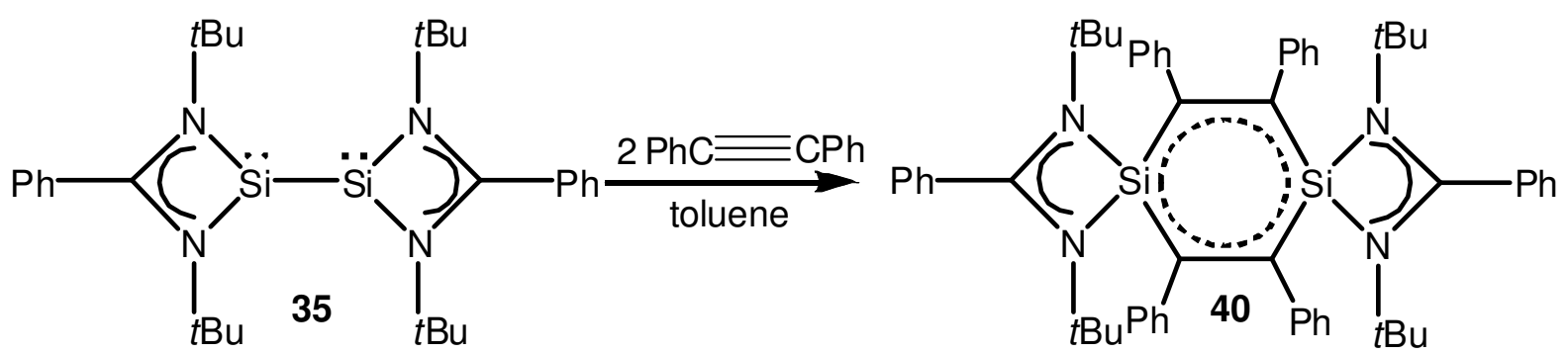

Scheme 5.1 Preparation of $\mathbf{4 0 .}$ 
The molecular structure of $\mathbf{4 0}$ was unequivocally determined by single crystal X-ray diffraction. ${ }^{44}$ The molecular structure of $\mathbf{4 0}$ is shown in Figure 5.1. $\mathbf{4 0}$ crystallized in the trigonal space group $P 3_{1} 21$. The structure revealed that both $\mathrm{Si}$ atoms show distorted tetrahedral geometry. The two nitrogen atoms from the amidinato ligands occupy the two sites and the remaining two sites are engaged with two carbon atoms. The 1,4-disilabenzene ring is almost planar, which is an important factor in contributing the enhanced stability of 40. A $C_{2}$ axis passes through the center of the disilabenzene ring. Another important feature is that the four-membered amidinato rings are oriented almost perpendicular to the six-membered ring probably due to avoid steric hindrance. The Si-C bond distance is $1.800(3) \AA$ which is shorter than the Si-C single bond length reported in the literature $(1.86 \AA-1.93 \AA)^{59}$ and longer than that of the $\mathrm{Si}-\mathrm{C}$ double bond found in $(\mathrm{TMS})_{2} \mathrm{Si}=\mathrm{C}(\mathrm{OTMS}) \mathrm{Ad}(1.764 \AA)\left(\mathrm{Ad}=\right.$ adamantyl). ${ }^{12}$ The Si-C bond length matches excellently with that of Sekiguchi's 1,2-disilabenzene derivative $(1.80 \AA$ and $1.79 \AA) .{ }^{60}$ The $\mathrm{C}$-C bond distance in the 1,4 -disilabenzene ring is 1.415(4) $\AA$, which is slightly longer than the normal $\mathrm{C}=\mathrm{C}$ double bond length $(1.34 \AA) .{ }^{91}$ 


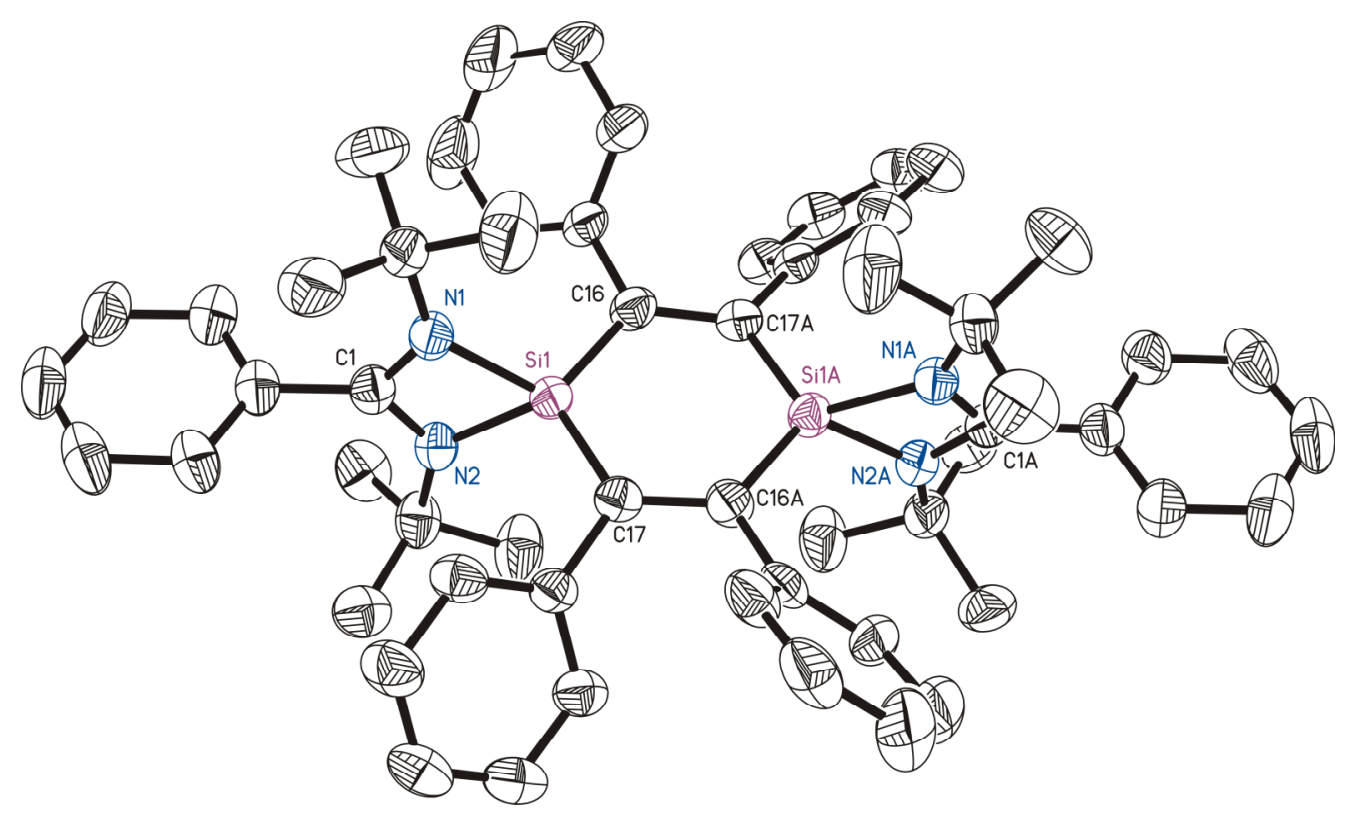

Figure 5.1 Molecular structure of 40. Anisotropic displacement parameters are depicted at the $50 \%$ probability level. Hydrogen atoms are omitted for clarity. Selected bond length $(\AA)$ and bond angles (deg): $C(16)-C(17) \# 1$ 1.415(4), $C(16)-C(18)$ 1.484(11), $C(16)-S i(1)$ 1.800(3), C(17)- (16)\#1 1.415(4), C(17)-C(24) 1.490(3), C(17)-Si(1) 1.799(2), N(1)-Si(1) 1.861(2), N(2)-Si(1) 1.859(2); N(1)-C(1)-N(2) 106.5(2), N(1)-C(1)-C(2) 127.1(3), N(2)-C(1)$\mathrm{C}(2)$ 126.4(2), N(1)-C(1)-Si(1) 53.29(13), N(2)-C(1)-Si(1) 53.23(13), C(2)-C(1)-Si(1) 178.2(2), C(17)\#1-C(16)-C(18') 118.6(6), C(18)-C(16)-Si(1) 117.6(5), C(16)\#1-C(17)-C(24) 119.6(2), $\mathrm{C}(16) \# 1-\mathrm{C}(17)-\mathrm{Si}(1)$ 123.18(17), C(24)-C(17)-Si(1) 116.88(18), C(1)-N(1)-C(8) 131.7(2).

In order to gain further insight into the electronic structure of $\mathbf{4 0}$ full DFT gas phase optimizations and frequency calculations were carried out for the singlet and for the triplet states of $\mathbf{4 0}$ at the UB3LYP/6-311G* level of theory. ${ }^{82}$ No negative frequencies were observed. The proper ground state was determined for both singlet and triplet state for $\mathbf{4 0}$ and it is found that the singlet state is energetically preferred over the triplet state with an energy difference of $10.61 \mathrm{kcal} / \mathrm{mol}$. The bond lengths for the singlet state of $\mathbf{4 0}$ compare well with the experimental ones ( $\mathrm{Si}-\mathrm{C}: 1.81, \mathrm{C}-\mathrm{C}: 1.43 \AA$ ). In the triplet state of $\mathbf{4 0}$ the $1,4-$ disilabenzene ring is more distorted and thus less planar than for the singlet state (average deviation from plane: 0.0815 and $0.0193 \AA$, respectively), which also leads to a shorter $\mathrm{Si}-\mathrm{Si}$ 
distance (3.33 vs. $3.39 \AA)$. The Si-C (1.82 $\AA$ ) and the C-C (1.44 $\AA$ ) bond lengths are in the same range as in the singlet state.

To verify the substituent effect in the stabilization of 1,4-disilabenzene, we replaced the phenyl group of the disilabenzene ring by hydrogen atoms (40a) and performed the same DFT calculation at the same level of theory. In this case also the singlet case is energetically favored over the triplet state with an energy gap of $7.49 \mathrm{kcal} / \mathrm{mol}$. For the singlet state of $\mathbf{4 0 a}$ the Si-C and C-C bond lengths are $1.79 \AA$ and $1.41 \AA$. The 1,4-disilabenzene ring is more planar than in $\mathbf{4 0}$ (mean deviation from plane: $0.0000 \AA$ ). For the triplet state of $\mathbf{4 0 a}$ one of the terminal phenyl rings is rotated against the molecular plane (i.e. the second terminal phenyl ring and the 1,4-disilabenzene ring) by about $40^{\circ}$, which leads to an elongation of the corresponding $\mathrm{Si}-\mathrm{C}$ bond to $1.85 \AA$ whereas the $\mathrm{Si}-\mathrm{C}$ bond length on the other side is $1.81 \AA$. The C-C bond length is slightly shorter than in the singlet state $(1.38 \AA)$.

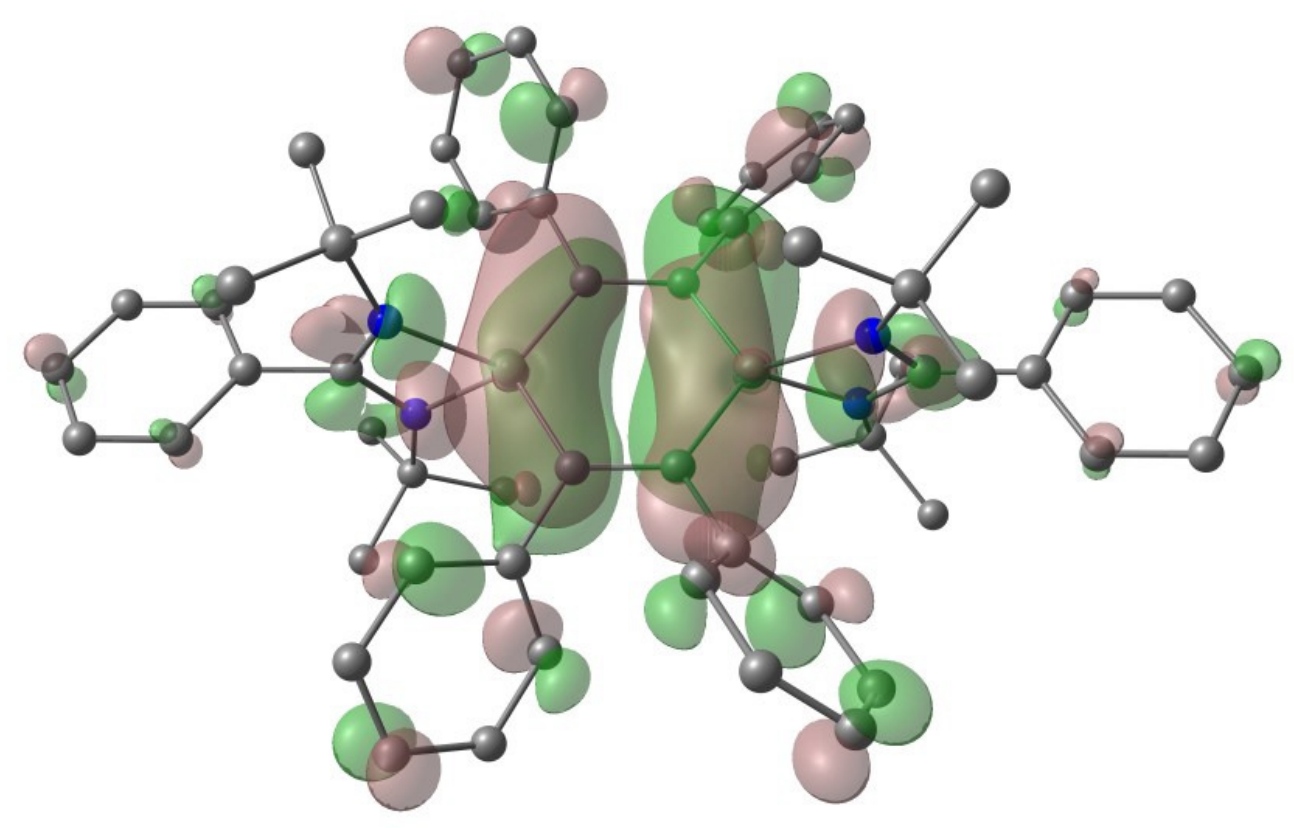

Figure 5.2 Highest occupied molecular orbital of compound 40. Hydrogen atoms have been omitted for clarity. Positive (green) and negative (red) isosurface representation at 0.02 a.u.. 
Also, for the theoretical results a possible diradical character for the 1,4-disilabenzene rings of 40 and 40a was investigated. However, none of the computational measures indicate a diradical. ${ }^{112}$ For both compounds the LUMO occupation number (calculated from natural orbitals) is zero, while the HOMO is doubly occupied. The spin density is zero, there is no spin contamination and the restricted closed shell calculation results in the same energy as the open shell unrestricted calculation. Moreover, the energy differences between the singlet and triplet states (40: $10.61 \mathrm{kcal} / \mathrm{mol}, \mathbf{4 0 a}: 7.49 \mathrm{kcal} / \mathrm{mol})$ and the HOMO-LUMO gaps (40: 34.67 $\mathrm{kcal} / \mathrm{mol}$, 40a: $41.09 \mathrm{kcal} / \mathrm{mol}$ ) are not small enough to indicate a diradical character.

To investigate if the character of the 1,4-disilabenzene rings in $\mathbf{4 0}$ and $\mathbf{4 0 a}$ is aromatic, the nucleus independent chemical shifts (NICS) at $1 \AA$ above the ring centers were calculated. ${ }^{113}$ 40 has a NICS(1) value of -3.64 which shows that it is slightly aromatic and 40a has a NICS(1) value of -5.17 , which indicates an even more aromatic character. These findings are also supported by the higher planarity of the 1,4-disilabenzene ring for $\mathbf{4 0 a}$ compared to that of 40. In addition, the highest occupied molecular orbitals with a nodal plane in the ring plane clearly resemble the equivalent orbitals (i.e. the $\pi$-MOs constructed from the carbon $\mathrm{p}_{\mathrm{z}^{-}}$ orbitals) from benzene in shape and symmetry, however, the energetic degeneracy of the HOMO is lost.

\subsection{Conclusion}

In conclusion, we were able to isolate 1,4-disilabenzene, which was elusive so far. Furthermore, NICS calculations showed that the compound has some aromatic character. The synthesis and isolation of room temperature stable 1,4-disilabenzene will open the way for the new developments in chemistry of aromatic silicon compounds. 


\section{Chapter 6}

\section{Synthesis, Structural Elucidation, Theoretical}

\section{Investigation and Reactivity Studies of a Bis-} germylene with a $\mathrm{Ge}(\mathrm{I})-\mathrm{Ge}(\mathrm{I})$ bond

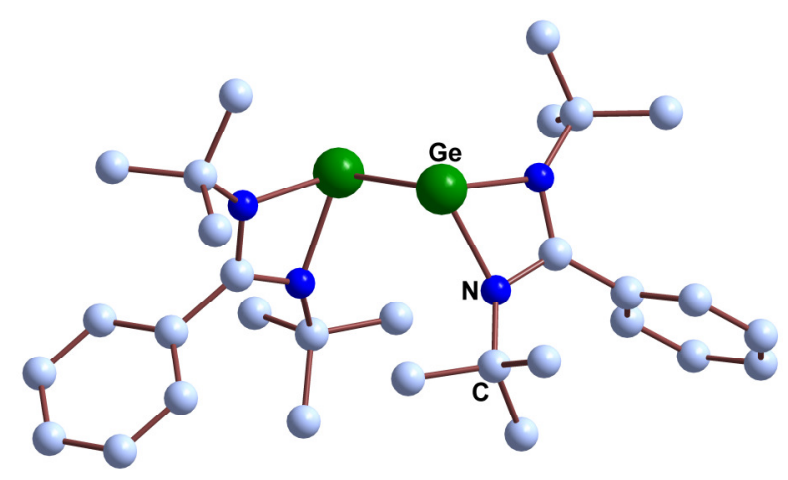

\section{Abstract}

The reduction of the chloride $\left[\mathrm{PhC}(\mathrm{N} t \mathrm{Bu})_{2}\right] \mathrm{GeCl}$ (41) with potassium in THF affords the reddish crystals of $\left[\mathrm{PhC}(\mathrm{N} t \mathrm{Bu})_{2}\right]_{2} \mathrm{Ge}_{2}$ (42). The molecule of 42 contains a $\mathrm{Ge}-\mathrm{Ge}$ bond. The X-ray structure and DFT calculation indicate that the Ge-Ge bond possesses unusual gauchebent geometry and there is no multiple bond character in $\mathbf{4 2}$. The Ge-Ge bond length in $\mathbf{4 2}$ is $2.570 \AA$ A. Furthermore 42 was treated with azobenzene and $\mathrm{Fe}_{2}(\mathrm{CO})_{9}$ to afford $\mathbf{4 4}$ and $\mathbf{4 5}$ respectively. In the former case cleavage of the Ge-Ge bond was observed, whereas in the latter case the Ge-Ge bond remains intact during the reaction. 


\subsection{Introduction}

Clemens Winkler, who discovered germanium in 1886, reported on the characteristics of elements:

\section{The world of chemical events}

Resembles a stage on which an

Unbroken succession of scenes is played out,

The cast consists of the elements,

Each is assigned its unique role,

Be it that of walk-on or principal character.

Residing between silicon and tin, germanium has become during the past decades a principal character in main group chemistry without any doubt. Alkines analogue of germanium was first achieved by Power and his co-workers in 2002. ${ }^{17}$ Inspection of structural data affords the central Ge-Ge bond distance to be 2.28(6) A. Following this Jones et al. reported the amidinato and guanidinato stabilized $\mathrm{Ge}(\mathrm{I})$ dimer $\{\mathrm{Ge}(\mathrm{Piso})\}_{2}$ and $\{\mathrm{Ge}(\mathrm{Giso})\}_{2}$, [Piso $=$ $(\mathrm{ArN})_{2} \mathrm{Ct} \mathrm{Bu}_{2}$, Giso $\left.=(\mathrm{ArN})_{2}-\mathrm{CNi} \mathrm{Pr}_{2}, \mathrm{Ar}=2,6-i \mathrm{Pr}_{2} \mathrm{C}_{6} \mathrm{H}_{3}\right] .{ }^{20 \mathrm{~b}}$ These compounds also possess Ge-Ge single bonds with lone pair of electrons on germanium atoms and exhibit trans-bent geometry. This is explicit from the introduction of this dissertation that the kinetic stabilization of the labile $\mathrm{Ge}_{2}$ core using the sterically demanding substituents is a feasible strategy to produce a stable alkine analogue of germanium. Our success in stabilizing the $\mathrm{Si}(\mathrm{I})$ dimer prompted us to study the alkine analogue of germanium. Earlier the amidinato ligand served to stabilize the $\mathrm{Si}(\mathrm{I})$ dimer. Therefore, we were most intrigued to verify whether amidinato ligand with $t \mathrm{Bu}$ substituents on nitrogen atoms is capable of stabilizing the low oxidation state of germanium and it seemed that the ligand worked perfectly. 


\subsection{Synthesis of Chloro germylene and its reduction}

The reaction of tert-butylcarbodiimide $(t \mathrm{BuN}=\mathrm{C}=\mathrm{N} t \mathrm{Bu})$ with one equivalent of $\mathrm{PhLi}$ in diethyl ether followed by treatment with $\mathrm{GeCl}_{2} \cdot$ dioxane afforded $\left[\mathrm{PhC}(\mathrm{N} t \mathrm{Bu})_{2}\right] \mathrm{GeCl}(\mathbf{4 1}$; Scheme 6.1). Compound 41 was obtained as colorless crystalline solid in $75 \%$ yield and its structure was confirmed by NMR spectroscopy, EI-MS spectrometry, and elemental analysis. The ${ }^{1} \mathrm{H}$ NMR spectrum of compound $\mathbf{4 1}$ shows a singlet at $1.08 \mathrm{ppm}$ for the 18 protons of two $t \mathrm{Bu}$ groups and another multiplet for 5 aromatic protons $(7.48-7.56 \mathrm{ppm})$. The most abundant ion peak in the EI-MS spectrum appeared at $m / z$ 338.5.

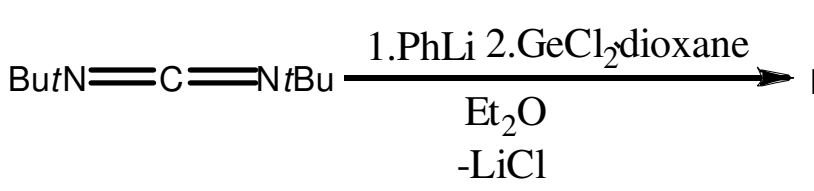

$-\mathrm{LiCl}$

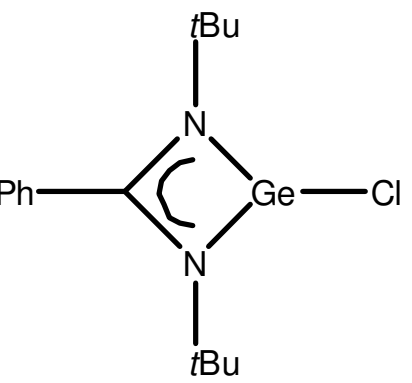

41

Scheme 6.1. Synthesis of 41

Maintaining a toluene solution of $\mathbf{4 1}$ overnight at $-32{ }^{\circ} \mathrm{C}$ resulted in colorless single crystals suitable for X-ray structural analysis. Compound $\mathbf{4 1}$ crystallizes in the monoclinic space group $C 2 / c$ (Figure 6.1). The Ge(II) center exhibits distorted tetrahedral geometry as the sum of the bond angles is $248^{\circ}$ which is significantly smaller than $360^{\circ}$. The two sites of the Ge(II) center are occupied by the $\mathrm{N}$ atoms from the amidinato ligand and the other site is occupied by a chlorine atom. The lone pair of the Ge(II) occupies the remaining coordination site. The structure is very similar to the structures of the recently reported amidinato and guanidinato germanium(II) chlorides. ${ }^{20 \mathrm{~b}}$ 


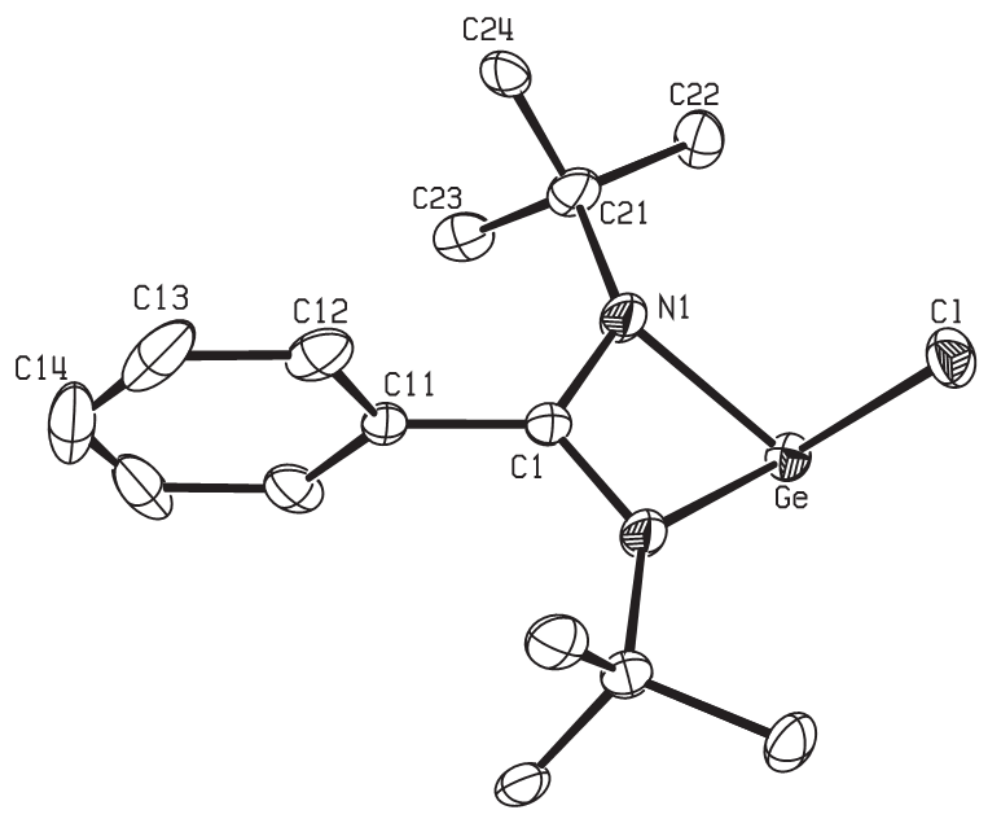

Figure 6.1. ORTEP view (30\% ellipsoid probability) of compound 41 . Hydrogen atoms have been removed for clarity. Selected bond distances $(\AA)$ and bond angles (deg) $\mathrm{Ge}(1)-\mathrm{Cl}(1)$ 2.2572(13) $\mathrm{Ge}(1)-\mathrm{N}(1) 2.060 \pm 0.012(2), \mathrm{N}(1)-\mathrm{C}(1)$ 1.330(3), N(1) $-\mathrm{C}(21)$ 1.480(3), $\mathrm{C}(1)$ $-\mathrm{C}(11)$ 1.483(4), N(1) - Ge(1) - N(1) 63.22(11), N(1) - Ge(1) - Cl(1) 92.10(8), N(1) - C(1) $\mathrm{N}(1)$ 108.6(3), $N(1)-C(1)-C(11) 125.72(13)$

Treatment of $\mathbf{4 1}$ with 1.5 equivalents of finely divided potassium in THF for 48 hours afforded a deeply colored solution of germanium dimer $\left[\mathrm{PhC}(\mathrm{N} t \mathrm{Bu})_{2}\right]_{2} \mathrm{Ge}_{2}$ (42; Scheme 6.2). Recrystallization of the crude product in toluene gave reddish crystals of germanium (I) dimer with $35 \%$ yield. Compound $\mathbf{4 2}$ was isolated as a reddish crystalline solid with good solubility in solvents such as diethyl ether, toluene, and THF. Moreover it is stable in solution or in the solid state at room temperature in an inert atmosphere. It has been characterized by spectroscopic methods, and X-ray crystallography.

The ${ }^{1} \mathrm{H}$ NMR spectrum of compound $\mathbf{4 2}$ shows a singlet at $1.16 \mathrm{ppm}$ for the 36 protons of four $t \mathrm{Bu}$ groups and another multiplet for 10 aromatic protons $(7.34-7.39 \mathrm{ppm})$. So there is a very little shift in the ${ }^{1} \mathrm{H}$ NMR spectrum from the starting material. The most abundant ion peak in the EI-MS spectrum appeared at $m / z 608.5$. 


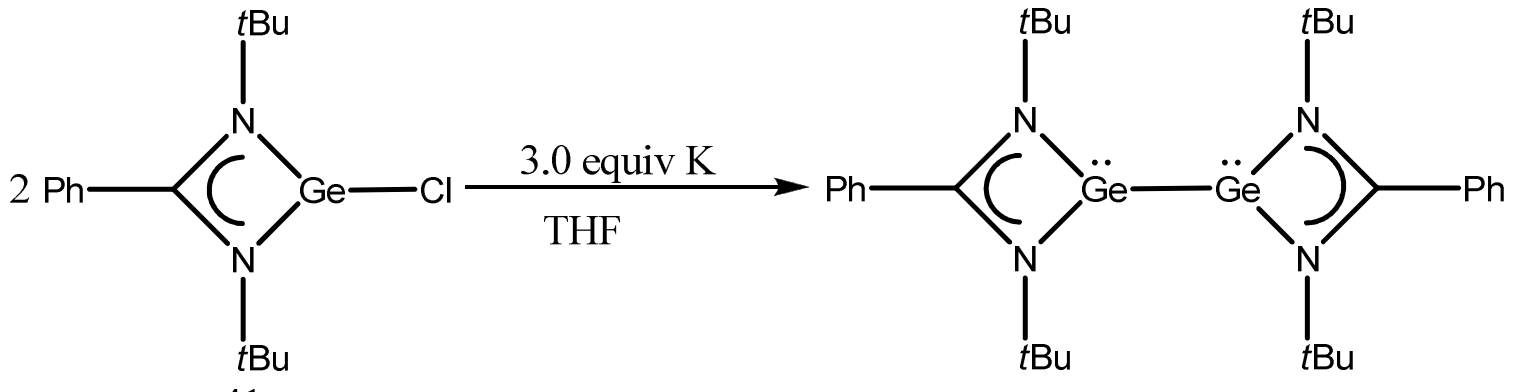

42

Scheme 6.2. Synthesis of 42

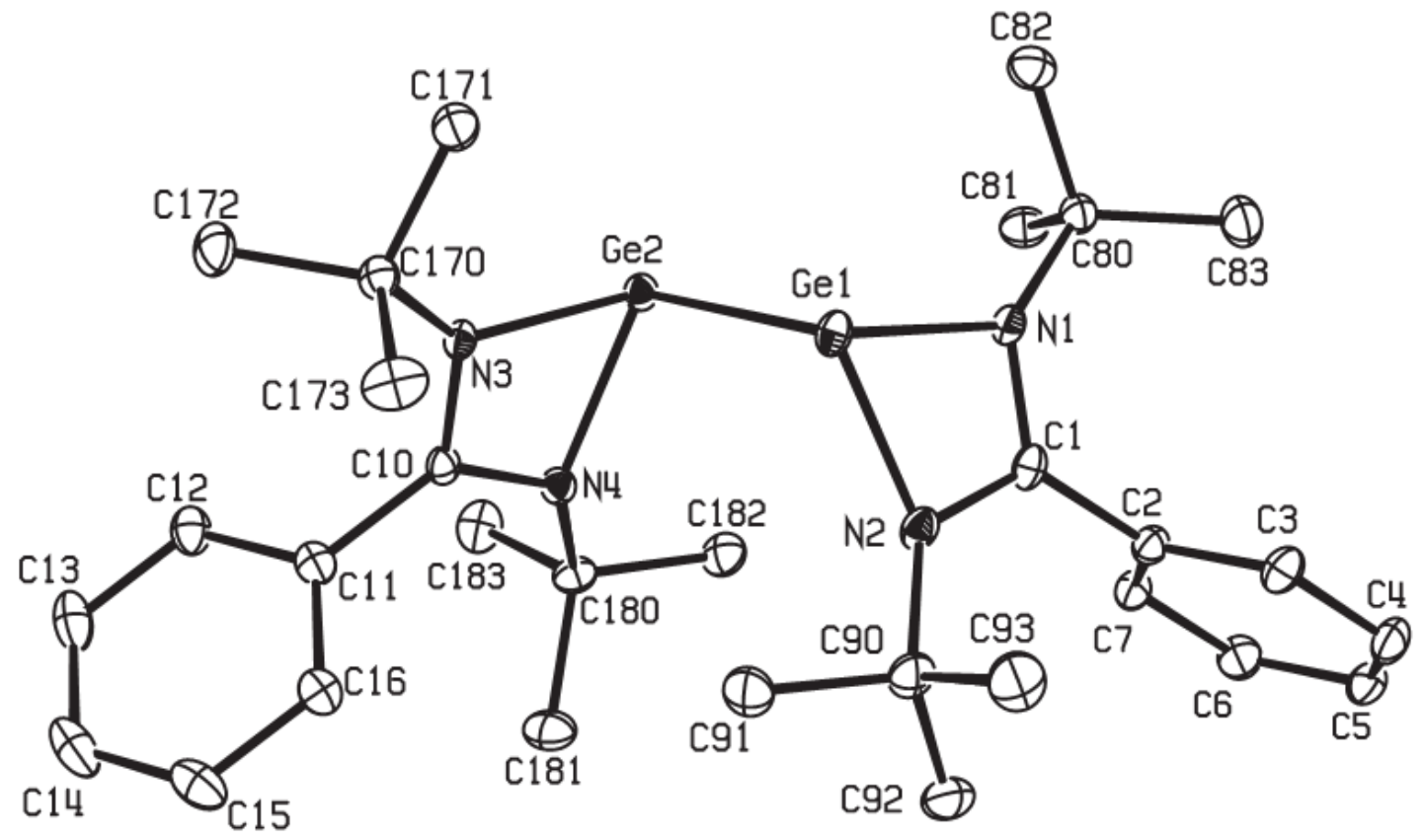

Figure 6.2. ORTEP view (30\% ellipsoid probability) of compound 42 . Hydrogen atoms have been removed for clarity. Selected bond distances $(\AA)$ and bond angles (deg) $\mathrm{Ge}(1)-\mathrm{N}(2)$ 2.005(2), Ge(1) - N(1) 2.026(2), Ge(2) - N(3) 2.020(2), Ge(2) - N(4) 2.014(2), Ge(1) - Ge(2) 2.569(5), $C(1)-N(1)$ 1.326(4), $C(1)-N(2)$ 1.337(4), $C(10)-N(3)$ 1.333(3), $C(10)-N(4)$ 1.338(3), N(1) - C(80) 1.482(4), N(2) - C(90) 1.479(4), N(3) - C(190) 1.481(4), N(4) $\mathrm{C}(180)$ 1.475(4), N(2) - Ge(1) - N(1) 64.65(9), N(2) - Ge(1) - C(1) 32.54(9), N(1) - Ge(1) $\mathrm{C}(1) 32.27(9), N(2)-\mathrm{Ge}(1)-\mathrm{Ge}(2)$ 103.55(7), N(1) -Ge(1) - Ge(2) 97.84(7), C(1) - Ge(1) $\mathrm{Ge}(2)$ 105.09(6), N(4) - Ge(2) - N(3) 64.85(9), N(4) - Ge(2) - C(10) 32.58(9), N(3) - Ge(2) $\mathrm{C}(10)$ 32.44(9), N(4) - Ge(2) - Ge(1) 104.62(7), N(3) - Ge(2) - Ge(1) 98.17(6), C(10) - Ge(2) $-\mathrm{Ge}(1) 105.96(6)$. 
The molecular structure of $\mathbf{4 2}$ is shown in Figure 6.2. Compound $\mathbf{4 2}$ crystallizes in the orthorhombic space group $P_{b c a}$. The coordination environment of one of the Ge(I) atoms exhibits a distorted tetrahedral geometry (Figure 6.2). The coordination sites of the $\mathrm{Ge}(\mathrm{I})$ centers are each occupied by the $\mathrm{N}$ atoms of the amidinato ligand and by the other $\mathrm{Ge}(\mathrm{I})$ atom. The lone pair of electrons at the Ge(I) occupies the remaining coordination site of the tetrahedron. The Ge-Ge bond length in $\mathbf{4 2}$ is $2.570 \AA$ which is very close to the single $\mathrm{Ge}-\mathrm{Ge}$ interaction $(2.61 \AA)$ but significantly longer than for typical digermenes, $\mathrm{R}_{2} \mathrm{GeGeR}_{2}(2.21-$ $2.51 \AA)$ and the two structurally characterized digermines $(2.2850 \AA \text { and } 2.2060 \AA)^{17}$ which proves that there is no multiple bond character in $\mathbf{4 2}$.

\subsection{Theoretical Investigation of $\left[\mathrm{PhC}(\mathrm{N} t \mathrm{Bu})_{2}\right]_{2} \mathrm{Ge}_{2}$}

In order to elucidate the nature of the Ge-Ge interaction, we performed DFT calculations on the isolated gauche species 42. Calculations were performed at both B3LYP/6-31G** and BP86/6-31G** levels of theory. Structures and energies are reported in B3LYP level if otherwise not mentioned. The DFT optimized geometry $\mathbf{4 3}$ shows a close resemblance with the geometry of $\mathbf{4 2}$ (Figure 6.3 and Figure 6.4) though the Ge-Ge bond length is overestimated (2.702 $\AA$ in $\mathbf{4 3}$ vs. $2.570 \AA$ in $\mathbf{4 2}$ ) and there are subtle differences in average Ge-N distances (2.091 $\AA$ in 43 vs. $2.015 \AA$ in 42 ). Moreover, the C1-Ge1-Ge2'-C10', dihedral angle has increased by $8.1^{\circ}\left(113.0^{\circ}\right.$ in $\mathbf{4 3}$ vs. $104.9^{\circ}$ in $\left.\mathbf{4 2}\right)$ during the course of the optimization.

Figure 6.4, (a) shows the Kohn-Sham (KS) HOMO of the optimized structure of $\mathbf{4 3}$ which is largely comprised of $\sigma$-bonding interaction between the Ge $p$-orbitals whereas in the KSLUMO (b) there is a strong Ge-Ge $\pi_{\mathrm{y}}$ bonding overlap with an additional $\mathrm{Ge}-\mathrm{N} \pi^{*}$ interaction (Figure 6.4). Similar types of frontier orbitals are reported by Jones and his 
coworkers in their theoretical investigations of trans-bent Ge(I) dimers. ${ }^{20 \mathrm{~b}}$ Weinhold's NBO analysis indicates a strong Ge-Ge bonding interaction in $\mathbf{4 3}$, with high $p$-character $(s$ character $14.4 \%, p$-character $85.3 \%$ ) and a Wiberg bond index of 0.915 . The Ge-N bonds are highly polarized (NPA charges: Ge; $0.524 e$ and N (mean); -0.713 $e$ ) with an average bond order of 0.431 .

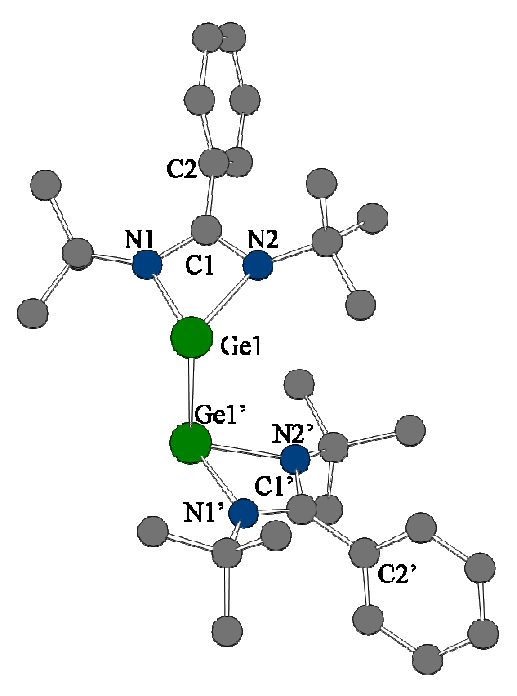

Figure 6.3. B3LYP/6-31G** optimized structure 43 (hydrogens are omitted for clarity) with selected bond lengths (in $\AA$ ) and angles (in deg). Ge1-Ge1' 2.702, Ge1-N1 (Ge1'-N1') 2.094, Ge1-N2 (Ge1'-N2') 2.091, N1-C1 (N1'-C1') 1.335, N2-C1 (N2'-C1') 1.345, C2Ge1-Ge1' 106.5, C2'-Ge1'-Ge1 106.5, C1-Ge1-Ge1'-C1' 113.0.

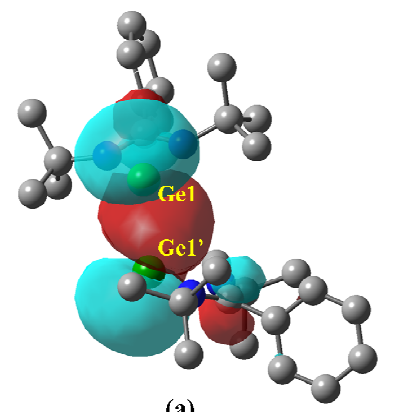

(a)

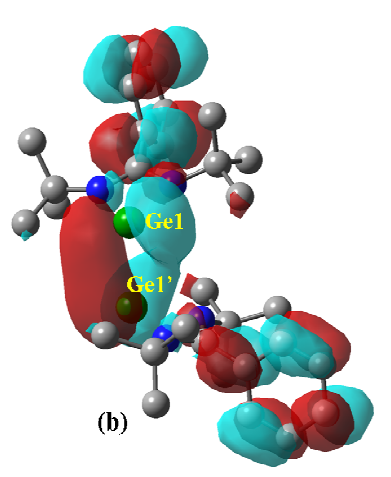

Figure 6.4. Frontier orbitals of $\mathbf{4 3}$ (isodensity value $=0.002$ electron/bohr ${ }^{3}$ ) (a) KS-HOMO ($3.564 \mathrm{eV})$ (b) KS-LUMO (-0.598 eV). 


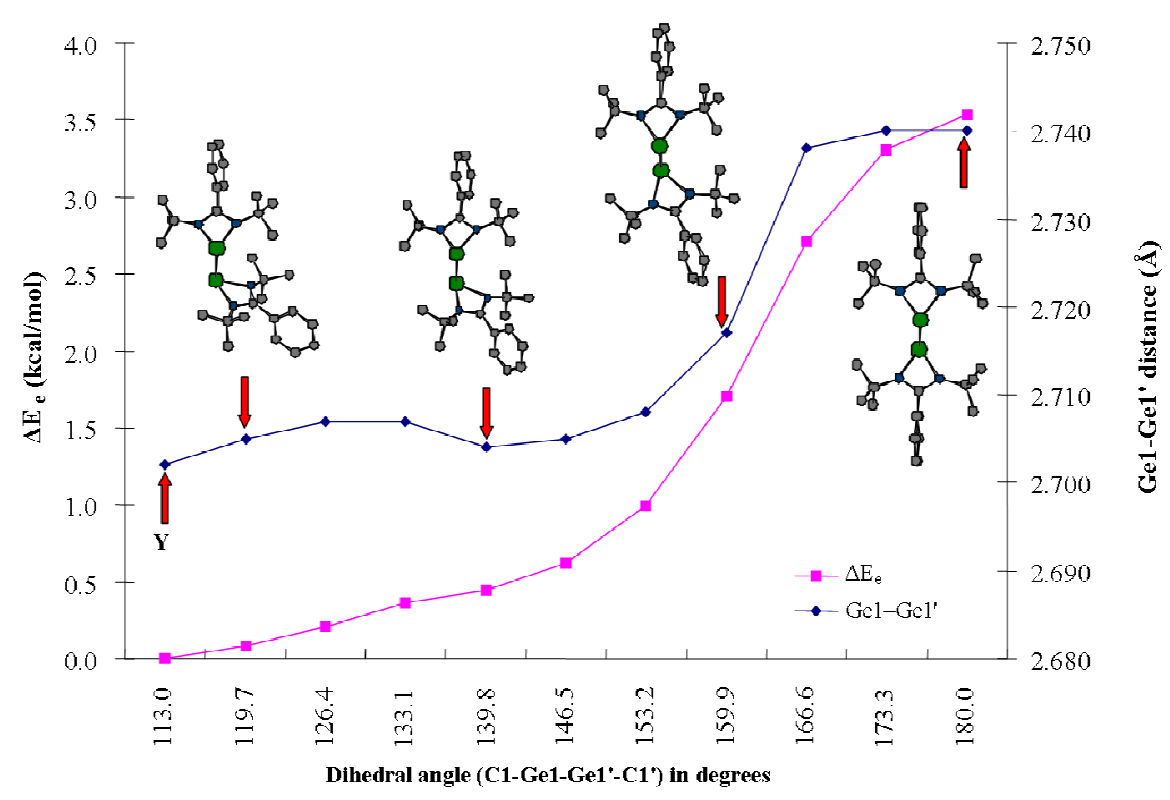

Figure 6.5. Potential energy scan for isomerization of $\mathbf{4 3}$ with respect to C1-Ge1-Ge1'-C1' dihedral angle changes at B3LYP/6-31G** level of theory (refer text).

Unlike the previous studies, which investigated on both the structural and electronic features of trans-bent group 14 elements, herein we report a similar theoretical characterization of a gauche-bent $\mathrm{Ge}(\mathrm{I})$ dimer 43 . We were curious to understand the relative stability of the optimized gauche-bent structure with respect to its trans-variant. To this end, we performed a relaxed potential energy scan of the $\mathrm{C} 1-\mathrm{Ge} 1-\mathrm{Ge} 1{ }^{\prime}-\mathrm{C} 1$ ' dihedral angle from $\mathbf{4 3}$. Figure 6.5 shows the energy profile along with the concomitant change in dihedral angles and Ge1-Ge1' distances. The drastic increase in the Ge1-Ge1' distance occurs with the increase of the dihedral angle from roughly $153^{\circ}$ to $166^{\circ}$.

The end structure of the scan with trans oriented amidinate ligands (C1-Ge1-Ge1'-C1' $\left.180^{\circ}\right)$ was fully optimized. Interestingly, the unconstrained optimization furnished the gauche-bent structure 43. In fact all similar attempts to optimize the trans configured geometry failed. This prompted us to conclude the absence of any trans-variant of conformer $\mathbf{4 2 .}$ 
Though previous theoretical investigations have pointed out the stability of the planar-trans conformation for the $\mathrm{Ge}, \mathrm{Sn}$, and $\mathrm{Pb}$ species they have also emphasized the effect of the bulky ligands in destabilizing the gauche conformer on steric grounds and experimentally utilizing this strategy to isolate the trans-bent conformer.

\subsection{Reactivity Studies of the Ge(I) Dimer}

Although the reactivity of digermines has been studied extensively, to the best of our knowledge the reactivity of a germanium(I) dimer where the Ge-Ge bond length has to be regarded a single bond has not been reported so far. Furthermore a detailed theoretical calculation proposed that $\mathbf{4 2}$ features two stereo active lone pairs, which prefer to remain non-bonded at each germanium atom. Fuelled by this unprecedented electronic structure we embarked to study the reactivity of $\mathbf{4 2}$. Unequivocally this is of great interest both to the organic and the organometallic chemists.

\subsubsection{Reaction with diazobenzene}

In order to derivatize 42, a reaction was performed with azobenzene $(\mathrm{PhN}=\mathrm{NPh})$ in a $1: 1$ molar ratio in toluene at room temperature (Scheme 6.3). This afforded 44 as colorless crystals in good yield. The product is stable under inert atmosphere and soluble in organic solvents like ether, toluene, and THF.

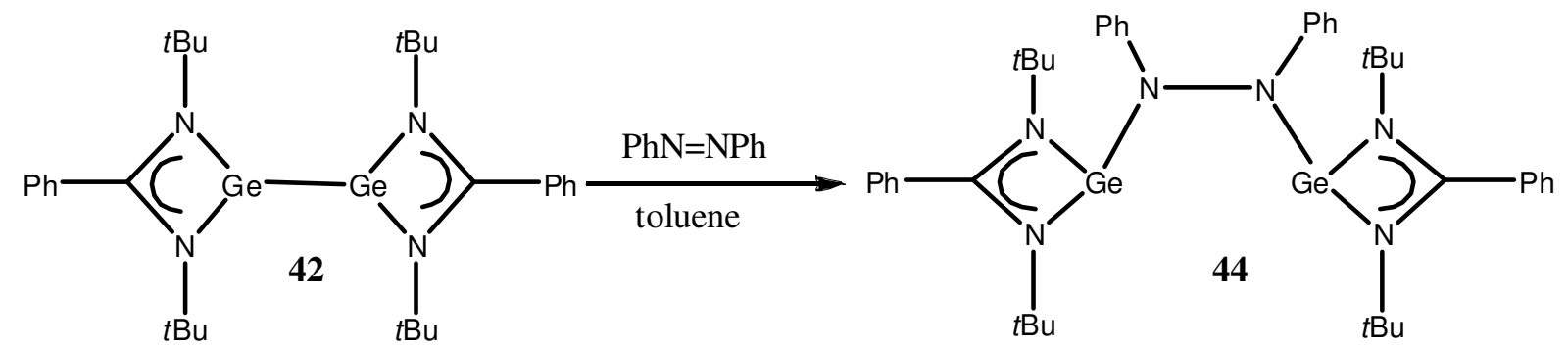




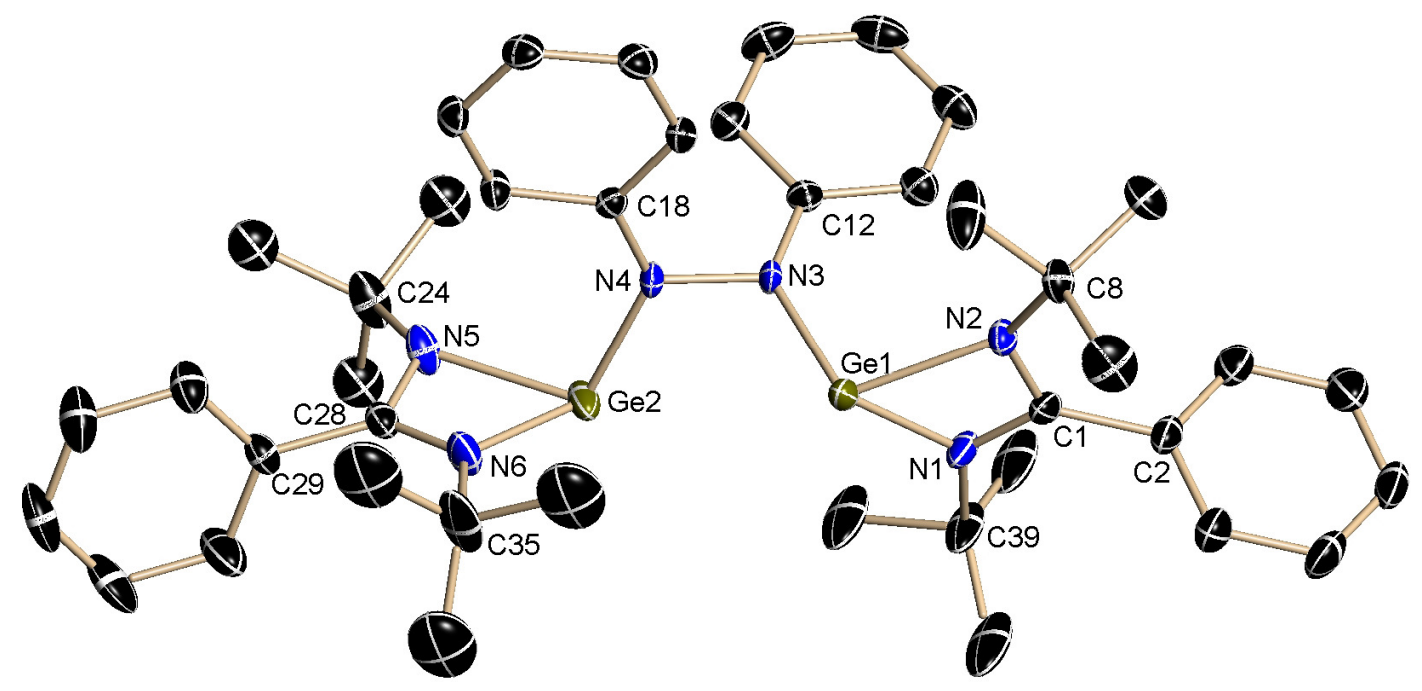

Figure 6.6. Anisotropic displacement parameters, depicted at the $50 \%$ probability level of 44. Hydrogen atoms and two toluene molecules are omitted for clarity. All four tBu-groups are rotationally disordered about the $\mathrm{N}-\mathrm{C}$-axis. Selected bond lengths $[\AA]$ ] and angles [ $\left.{ }^{\circ}\right]: \mathrm{N}(2)$ - Ge(1) 2.050(3), Ge(1) - N(3) 1.911(3), Ge(2) - N(4) 1.902(3), N(3) - C(12) 1.401(4), N(3) $\mathrm{N}(4)$ 1.436(4); $\mathrm{C}(28)-\mathrm{N}(5)-\mathrm{Ge}(2)$ 91.1(2), C(24) - N(5) - Ge(2) 133.1(2), C(28) - N(6) $\mathrm{Ge}(2)$ 92.7(2), $\mathrm{C}(35)-\mathrm{N}(6)-\mathrm{Ge}(2)$ 136.3(3), N(4) - Ge(2) - N(5) 105.6(13), N(4) - Ge(2) $\mathrm{N}(6)$ 103.6(14), $\mathrm{C}(12)-\mathrm{N}(3)-\mathrm{Ge}(1)$ 134.5(2), N(4) - N(3) - Ge(1) 110.8(2), C(18) - N(4) $\mathrm{Ge}(2)$ 132.7(2), N(3) - N(4) - Ge(2) 110.9(2), C(1) - N(1) - Ge(1) 93.0(2), C(39) - N(1) $\mathrm{Ge}(1)$ 135.4(3), $\mathrm{C}(1)-\mathrm{N}(2)-\mathrm{Ge}(1)$ 91.7(2), $\mathrm{C}(8)-\mathrm{N}(2)-\mathrm{Ge}(1)$ 134.2(2), N(3) - Ge(1) $\mathrm{N}(1)$ 104.4(13), N(3) - Ge(1) - N(2) 105.1(12), N(1) - Ge(1) - N(2) 64.1(12)

The molecular graph from the crystal structure determination of $\mathbf{4 4}$ is shown in Figure $6.6{ }^{44}$ Compound 44 crystallizes in the monoclinic space group Pbcn. The most striking result of the reaction is the unambiguous cleavage of the $\mathrm{Ge}-\mathrm{Ge}$ bond and the insertion of the substituted $\mathrm{N}_{2}$ motif of the azobenzene. The formal oxidation state of both germanium atoms in $\mathbf{4 4}$ is increased to $+\mathrm{II}$. The $\mathrm{N}-\mathrm{N}$ bond distance of 1.44 (4) $\AA$ is consistent with the interpretation as a single bond. ${ }^{114}$ In addition the $\mathrm{N}-\mathrm{C}$ bond distances remain unchanged. Therefore the reaction of 42 with $\mathrm{PhN}=\mathrm{NPh}$ has to be regarded as an oxidative addition with simultaneous Ge-Ge bond cleavage. As a result 44 is a molecular chain containing four elements each with a lone pair of electrons. Here it is worth to mention that a similar kind of Ge-Ge bond 
cleavage was reported by Power et al. when $\mathrm{RGeGeR}\left[\mathrm{R}=2,6-\mathrm{Trip}_{2} \mathrm{C}_{6} \mathrm{H}_{3}\right.$ (Trip = 2,6$\left.i \mathrm{Pr}_{2} \mathrm{C}_{6} \mathrm{H}_{3}\right)$ ] was reacted with azobenzene. ${ }^{115}$ In addition to that the $\mathrm{Ge}-\mathrm{N}$ bond lengths $(1.882(4)$ and $1.875(4) \AA)$ and N-N bond length $(1.45(3) \AA)$ in $\operatorname{RGe}\{(\mathrm{Ph}) \mathrm{NN}(\mathrm{Ph})\} \mathrm{GeR}$ coincide very well with those of $\mathbf{4 4}$. Both germanium atoms are tri-coordinate and exhibit each a distorted trigonal pyramidal geometry with a stereochemically active lone-pair at one apex. The three other sites at the germanium atom are occupied by the two nitrogen atoms of the amidinato ligand and one nitrogen atom of the bridging azobenzene. The coordination of the nitrogen atoms is almost planar with the angular sum $\Sigma^{\circ} \mathrm{N} 3,4$ of $359.8(2)^{\circ}$. The $\mathrm{Ge}-\mathrm{N}$ bond length $\left(1.905(3) \AA\right.$ ) is within the range found for other Ge (II) amides. ${ }^{114}$

Additionally, 44 was characterized by NMR spectroscopy, EI-MS spectrometry, elemental analysis, and furthermore by X-ray crystallography. The ${ }^{1} \mathrm{H}$ NMR spectrum of $\mathbf{4 4}$ exhibits three sets of resonances, one from the $t \mathrm{Bu}$ protons of the amidinato ligand and the second one of the phenyl rings of the same ligand, and the third one of the phenyl substituents of the azobenzene. In the EI-MS spectrum the molecular ion appeared as the most abundant peak with the highest relative intensity at $m / z, 790$.

\subsubsection{Reaction with diiron nonacarbonyl}

In the aforementioned experiment the $\mathrm{Ge}-\mathrm{Ge}$ bond in $\mathbf{4 2}$ was cleaved by insertion of azobenzene. But we were curious to see a reactivity where the $\mathrm{Ge}-\mathrm{Ge}$ bond will remain intact. In order to obviate such possibility and minimize the opportunity of the cleavage we wanted to employ the lone pair at the Ge(I) atom in bonding. According to Pearson's HSAB concept it can be considered as a soft base. ${ }^{116}$ So the simplest idea is to treat $\mathbf{4 2}$ with a soft Lewis acid and check whether it can form the Lewis acid-base adduct. Therefore, diironnonacarbonyl, $\mathrm{Fe}_{2}(\mathrm{CO})_{9}$, with iron in the formal oxidation state zero was judiciously chosen as a probe to investigate the reaction behavior. It is known to form metal complexes with N-heterocyclic 
silylene. ${ }^{69}$ In our case the reaction successfully affords the target complex, a unique example of a Lewis acid base adduct employing both $\mathrm{Ge}(\mathrm{I})$ centers with adjacent lone pairs as Lewis bases.

Treatment of $\mathbf{4 2}$ with two equivalents of $\mathrm{Fe}_{2}(\mathrm{CO})_{9}$ in THF for 1 day afforded $\mathbf{4 5}$ (Scheme 6.4). After the reaction the solvent was removed in vacuum and the residue extracted with toluene. The insoluble solid was filtered off and the filtrate was concentrated and stored at $30{ }^{\circ} \mathrm{C}$ in a freezer to yield red crystals of $\mathbf{4 5}$, suitable for the X-ray diffraction study.

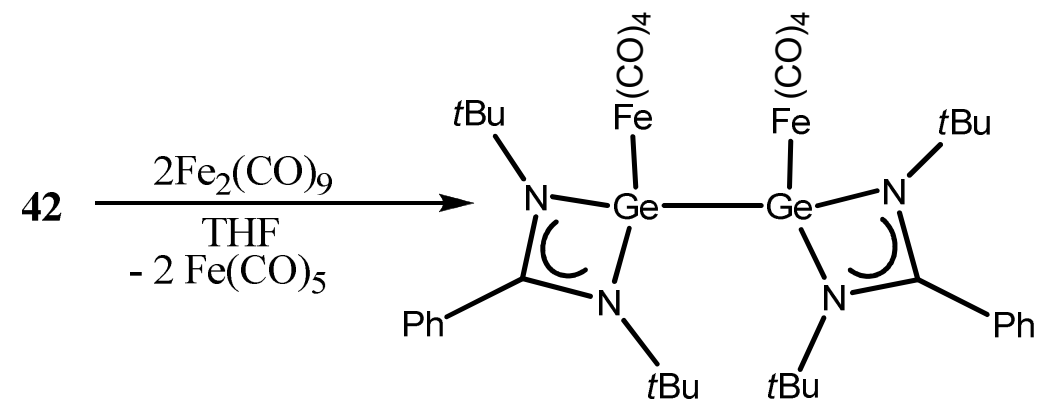

45

Scheme 6.4. Preparation of 45

The solid-state structure of $\mathbf{4 5}$ is shown in Figure $6.7 .^{44} \mathbf{4 5}$ crystallizes in the centrosymmetric monoclinic space group $C 2 / c$. The most striking feature of the structure is the unbroken $\mathrm{Ge}-$ Ge bond. This bond length is $2.55(5) \AA$, hence is reduced a little in comparison to 42 (2.57(5) $\AA$ ). In 45 , each Ge atom binds to the two nitrogen atoms from the monoanionic chelating amidinato ligand, to another $\mathrm{Ge}(\mathrm{I})$ atom, and to a $\mathrm{Fe}(\mathrm{CO})_{4}$ moiety, leaving the germanium atom four-coordinate in a distorted tetrahedral coordination geometry. Each Fe atom is centered in a trigonal bipyramidal polyhedron, four sites are occupied by carbonyl groups and one by a germanium atom. The $\mathrm{Fe}(\mathrm{I})-\mathrm{Ge}(\mathrm{I})$ bond distance in $\mathbf{4 5}$ is 2.34(4) $\AA$, which is very similar to those found in $\mathrm{LGe}(\mathrm{OH}) \mathrm{Fe}(\mathrm{CO})_{4}(2.33(1) \AA)\left(\mathrm{L}=\mathrm{HC}(\mathrm{CMeNAr})_{2}\right.$ with $\mathrm{Ar}=2,6-i$ $\left.\operatorname{Pr}_{2} \mathrm{C}_{6} \mathrm{H}_{3}\right)^{117 \mathrm{a}}$ and $\left[\eta^{3}-\left((\mu-t \mathrm{BuN})_{2}(\mathrm{SiMeN} t \mathrm{Bu})_{2}\right) \mathrm{GeFe}(\mathrm{CO})_{4}\right](2.348(1) \AA)^{117 \mathrm{~b}}$ and also slightly longer when compared with that of $\mathrm{LGe}(\mathrm{Cl}) \mathrm{Fe}(\mathrm{CO})_{4}\left(2.29(2) \AA ; \mathrm{L}=\mathrm{HC}\{(\mathrm{CMe})(\mathrm{NPh})\}_{2}\right){ }^{117 \mathrm{c}}$ 
But in all the mentioned examples the $\mathrm{Fe}(\mathrm{CO})_{4}$ fragment is bonded to $\mathrm{Ge}$ atoms in the formal oxidation state of $+\mathrm{II}$. To the best of our knowledge $\mathbf{4 5}$ is the first example where the $\mathrm{Fe}(\mathrm{CO})_{4}$ moiety is attached to a germanium atom in the formal oxidation state of $+\mathrm{I}$.

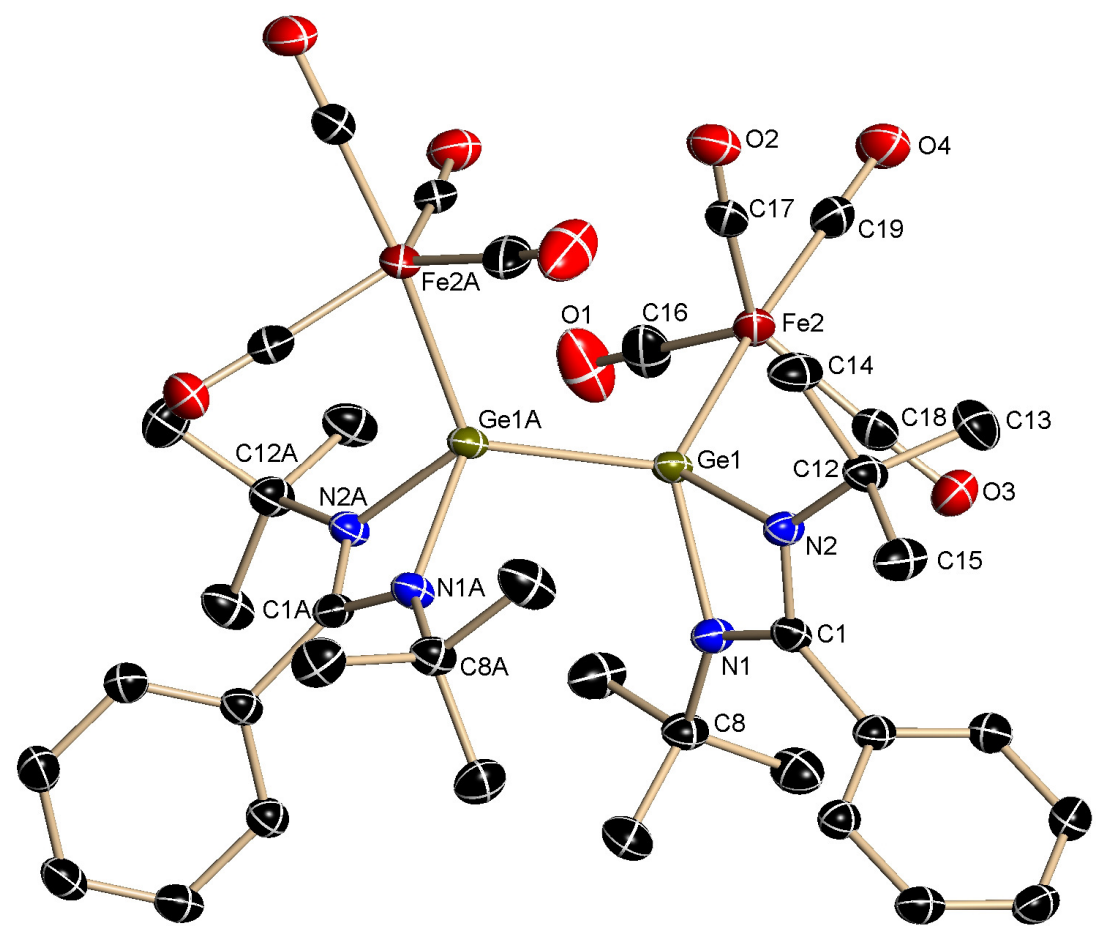

Figure 6.7. Anisotropic displacement parameters, depicted at the $50 \%$ probability level of 45. Hydrogen atoms are omitted for clarity. Selected bond lengths $[\AA]$ and angles [ ${ }^{\circ}: \mathrm{Ge}(1)-$ $\mathrm{Ge}(1 \mathrm{~A})$ 2.5512(5), $\mathrm{Ge}(1)-\mathrm{N}(1)$ 1.9623(16), $\mathrm{Ge}(1)-\mathrm{N}(2)$ 1.9817(16), $\mathrm{Fe}(2)-\mathrm{C}(16)$ 1.791(2), $\mathrm{Fe}(2)-\mathrm{Ge}(1)$ 2.3402(4); C(18)-Fe(2)-C(19) 92.34(9), N(1)-Ge(1)-N(2) 66.60(6), N(1)-Ge(1)$\mathrm{Fe}(2)$ 118.44(5), N(2)-Ge(1)-Fe(2) 114.94(5), N(1)-Ge(1)-Ge(1A) 111.66(5), N(2)-Ge(1)$\mathrm{Ge}(1 \mathrm{~A})$ 108.00(5), $\mathrm{Fe}(2)-\mathrm{Ge}(1)-\mathrm{Ge}(1 \mathrm{~A})$ 123.211(10).

Additionally 45 was characterized by mass spectrometry, NMR spectroscopy, elemental analysis. All obtained data are in accordance with the structure of $\mathbf{4 5}$. The ${ }^{1} \mathrm{H}$ NMR spectrum of $\mathbf{4 5}$ shows a singlet at $1.41 \mathrm{ppm}$ for the 36 protons of four $t \mathrm{Bu}$ groups and another multiplet for 10 aromatic protons (7.56-7.82 ppm). In the ${ }^{13} \mathrm{C}$ NMR spectrum the chemical shift of the carbonyl groups in $\mathbf{4 5}$ (220.56 ppm) is similar to that observed for the N-heterocyclic silylene complex with $\mathrm{Fe}(\mathrm{CO})_{4}{ }^{69}$ The molecular ion in the EI-MS spectrum appeared as the most 
Chapter 6. Synthesis, Structural Elucidation, Theoretical Investigation and Reactivity Studies of a Bis-germylene with a $G e(I)-G e(I)$ bond

abundant peak at $m / z$ 943. The carbonyl stretching frequencies of 45 comes at 2029 (m), 1974 (s), $1920(\mathrm{~s}) \mathrm{cm}^{-1}$.

\subsection{Conclusion}

We have prepared a germanium(I) dimer which is stabilized by bulky amidinate ligands. Theoretical studies confirm the dimer exhibits a gauche-bent geometry and the Ge-Ge bond shows no multiple bond character. We also performed further reactions with germanium(I) dimer. It shows strikingly two different types of reactivities with azobenzene and diironnonacarbonyl with and without the cleavage of the Ge-Ge bond respectively. 


\title{
Chapter7
}

\section{Synthesis of Monomeric Divalent Tin(II)}

\section{Compounds with Terminal Chloride, Amide, and}

\section{Triflate Substituents}

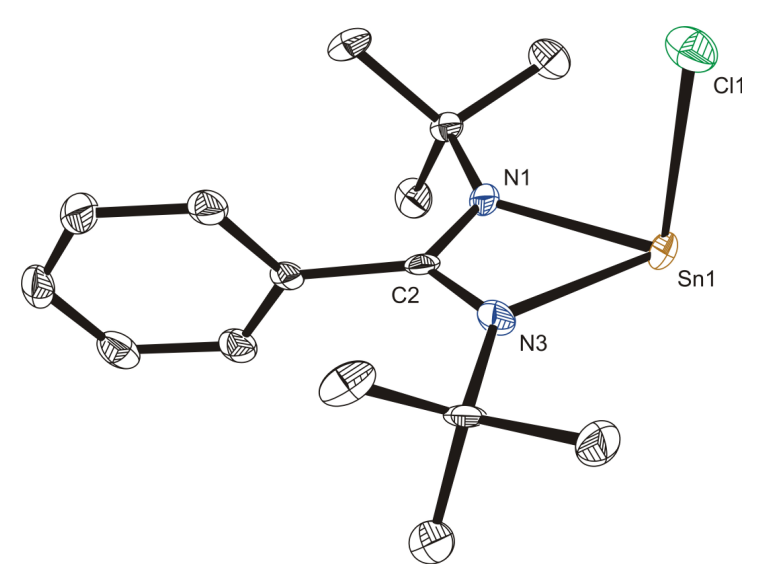

\begin{abstract}
Monomeric three-coordinate amidinato tin chloride $\left(\left(\mathrm{PhC}(\mathrm{N} t \mathrm{Bu})_{2} \mathrm{SnCl}\right)(\mathbf{4 6})\right.$ was prepared by the reaction of tert-butyl carbodiimide, phenyl lithium, and $\mathrm{SnCl}_{2}$. The metathesis reaction of 46 with $\mathrm{AgSO}_{3} \mathrm{CF}_{3}$ and $\mathrm{LiN}\left(\mathrm{SiMe}_{3}\right)_{2}$ afforded the formation of $\mathrm{PhC}(\mathrm{N} t \mathrm{Bu})_{2} \mathrm{SnOTf}$ $\left(\mathrm{Tf}=\mathrm{CF}_{3} \mathrm{SO}_{2}\right)(49)$ and $\mathrm{PhC}(\mathrm{N} t \mathrm{Bu})_{2} \mathrm{SnN}\left(\mathrm{SiMe}_{3}\right)_{2}$ (48). The reductive dehalogenation of 46 with K-selectride resulted in the formation of four-coordinate homoleptic tin, $\mathrm{Ph}_{2} \mathrm{C}_{2}(\mathrm{~N} t \mathrm{Bu})_{4} \mathrm{Sn}$ (49). Compounds 46, 47, 48, and 49 were characterized by single crystal structural analysis. Furthermore 46 was reacted with $\mathrm{Fe}_{2}(\mathrm{CO})_{9}$ to afford the stannylene iron carbonyl Lewis acidbase adduct (50).
\end{abstract}




\subsection{Introduction}

There is widespread interest in the chemistry of divalent derivatives of the heavier group 14 elements, due to their carbene-like properties. ${ }^{118}$ In contrast to carbenes and silylenes, the germylenes and stannylenes are less reactive due to the larger energy gap between the $s$ and $p$ orbitals. ${ }^{19}$ The germanium analogue of Arduengo's carbene ( $\left.t \mathrm{BuNCHCHN} t \mathrm{Bu}\right) \mathrm{Ge}$ was obtained by Herrmann et al. in $1992 .{ }^{120}$ In view of this, the present Ge(II) chemistry is very rich and much diversified with different types of germylene derivatives, ${ }^{121}$ which have been reviewed periodically. ${ }^{122}$ On the contrary the tin analogue of "Arduengo type carbene" is relatively scant despite the well-known inert pair effect, which proposes that divalent group 14 species should become more stable upon descending the group and was accomplished by Gudat and co-workers only in $2002 .{ }^{123 a}$ Following this Russell et al. very recently reported the synthesis and structural elucidation of five tin analogues of $\mathrm{N}$-heterocyclic carbene. ${ }^{123 \mathrm{~b}}$ Nonetheless there has been considerable interest over the past three decades in the chemistry of dialkyl and diaryl Sn(II) compounds following the pioneering studies by Lappert and coworkers. ${ }^{65 \mathrm{w}, 74 \mathrm{c}, 124}$ Stable tin(II) compounds of formula $\left(\mathrm{SnR}_{2}\right)_{1,2}$ and $\left(\mathrm{RSnX}^{1}\right)_{1,2}(\mathrm{R}=$ bulky ligand, $\mathrm{X}^{1}=$ halide) are well-characterized and are an abundant class of compounds. ${ }^{125}$ In contrast, derivatives of tin(II) of the type $\operatorname{Sn}\left(X^{2}\right) R$, where $X^{2}$ is a small ligand other than halide, have received much less attention. This is albeit surprising because these tin(II) derivatives can act as a good precursor for polymerization and catalysis reactions. It is also noteworthy that simple $\mathrm{Sn}(\mathrm{II})$ halides have important applications as promoters for Ptcatalyzed hydroformylation. ${ }^{126}$ Moreover owing to its low toxicity Sn(II) compounds are preferred for medical and pharmaceutical applications compared to any other metal ions, e.g. Sn(ethyl hexanoate $)_{2}$ is a permitted food additive in many countries. However, to the best of our knowledge, only few such compounds are known, including $\mathrm{Sn}\left(\mathrm{C}_{7} \mathrm{H}_{7}\right)\left[2,6-\left(\mathrm{CH}_{2} \mathrm{NMe}_{2}\right)_{2}-\right.$ 
Chapter 7. Synthesis of Monomeric Divalent Tin(II) Compounds with Terminal Chloride, Amide, and Triflate Substituents

$\left.\mathrm{C}_{6} \mathrm{H}_{3}\right],{ }^{125 \mathrm{e}} \quad\left[(n-\mathrm{Pr})_{2} \mathrm{ATI}\right] \mathrm{SnN}_{3} \quad\left(\right.$ where $\quad\left[(n-\mathrm{Pr})_{2} \mathrm{ATI}\right] \quad=\quad N-(n$-propyl $)-2-(n-$ propylamino)troponiminate $),{ }^{125 \mathrm{k}} \mathrm{Sn}\left[\mathrm{B}\left(\mathrm{C}_{6} \mathrm{~F}_{5}\right)_{4}\right] \mathrm{Cp},{ }^{127} \quad\left[\mathrm{Sn}\left(\mathrm{SO}_{3} \mathrm{CF}_{3}\right)\left\{\mathrm{N}\left(\mathrm{SiMe}_{3}\right)_{2}\right\}\right]_{2}{ }^{128}$, and $\left[\left\{\mathrm{HC}(\mathrm{CMeNAr})_{2}\right\} \mathrm{SnX}\right]\left(\mathrm{Ar}=2,6-i \mathrm{Pr}_{2} \mathrm{C}_{6} \mathrm{H}_{3}\right),\left(\mathrm{X}=\mathrm{Cl}, \mathrm{I}, \mathrm{N}\left(\mathrm{SiMe}_{3}\right)_{2}, \mathrm{Me}, \mathrm{F}, \mathrm{OTf}\right) .{ }^{28 \mathrm{~b}, 129}$ From these literature exploration it is understandable that there is plenty of room for new discoveries in tin(II) chemistry and we believe that base-stabilized heteroleptic stannylenes to be particularly attractive in extending the heavier group 14 analogues of carbene.

In order to explore the chemistry of three-coordinate tin, we turned our attention towards benz-amidinato ligand with $t \mathrm{Bu}$ substituent at the nitrogen atoms. Our experience in synthesizing few heteroleptic silylenes ( $\left.\mathrm{LSiX}, \mathrm{X}=\mathrm{Cl}, \mathrm{O} t \mathrm{Bu}, \mathrm{NMe}_{2}, \mathrm{P} t \mathrm{Bu}\right), \quad(\mathrm{L}=$ $\left.\mathrm{PhC}(\mathrm{N} t \mathrm{Bu})_{2}\right)^{25 \mathrm{a}, 37}$ and gauche-bent silicon(I) and germanium(I) dimer $\left(\left[\mathrm{PhC}(\mathrm{N} t \mathrm{Bu})_{2}\right]_{2} \mathrm{Si}_{2}\right.$, $\left.\left[\mathrm{PhC}(\mathrm{N} t \mathrm{Bu})_{2}\right]_{2} \mathrm{Ge}_{2}\right)$ by taking advantage of such a ligand prompted us to invesigate the chemistry of subvalent tin compounds with the same ligand. Herein, we report the preparation of $\mathrm{LSnCl}$ (46) $\left(\mathrm{L}=\mathrm{PhC}(\mathrm{N} t \mathrm{Bu})_{2}\right)$, and the resulting derivatives $\operatorname{LSn}\left(\mathrm{OSO}_{2} \mathrm{CF}_{3}\right)$ (47), $\mathrm{LSnN}(\mathrm{TMS})_{2}(\mathbf{4 8})$, and $\mathrm{L}_{2} \mathrm{Sn}(49)$. Furthermore 46 was reacted with $\mathrm{Fe}_{2}(\mathrm{CO})_{9}$ to afford the $\mathrm{LSnCl} \rightarrow \mathrm{Fe}(\mathrm{CO})_{4}(\mathbf{5 0})$ Lewis acid-base adduct.

\subsection{Synthesis and characterization aspect}

\subsubsection{Synthesis of $\left[\mathrm{PhC}(\mathrm{N} t \mathrm{Bu})_{2}\right] \mathrm{SnCl}$}

The reaction of tert-butylcarbodiimide with one equivalent of $\mathrm{PhLi}$ in diethyl ether followed by treatment with $\mathrm{SnCl}_{2}$ afforded $\left[\mathrm{PhC}(\mathrm{N} t \mathrm{Bu})_{2}\right] \mathrm{SnCl}$ (46; Scheme 7.1). Compound 46 was obtained as colorless crystalline solid in $75 \%$ yield and its structure was confirmed by NMR spectroscopy, EI-MS spectrometry, and elemental analysis. 
Chapter 7. Synthesis of Monomeric Divalent Tin(II) Compounds with Terminal Chloride, Amide, and Triflate Substituents

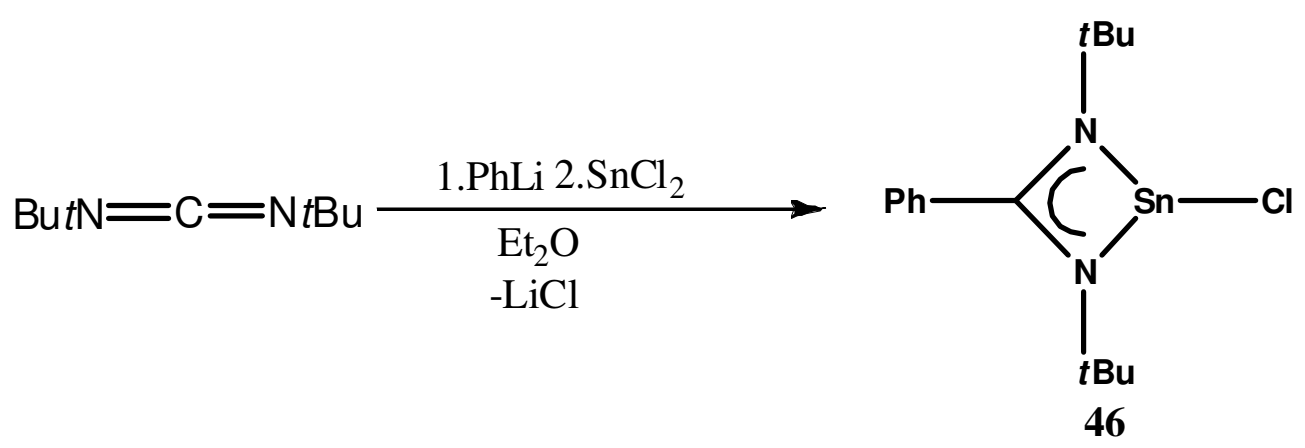

Scheme 7.1. Preparation of 46

The ${ }^{1} \mathrm{H}$ NMR spectrum of compound $\mathbf{4 6}$ shows a singlet at $1.08 \mathrm{ppm}$ for the 18 protons of two $t \mathrm{Bu}$ groups and another multiplet for 5 aromatic protons (7.48-7.56 ppm). 46 resonates at 29.6 ppm in the ${ }^{119} \mathrm{Sn}$ NMR spectrum. The molecular ion peak is observed with the highest relative intensity in the EI-MS spectrum at $m / z$ 385.5.

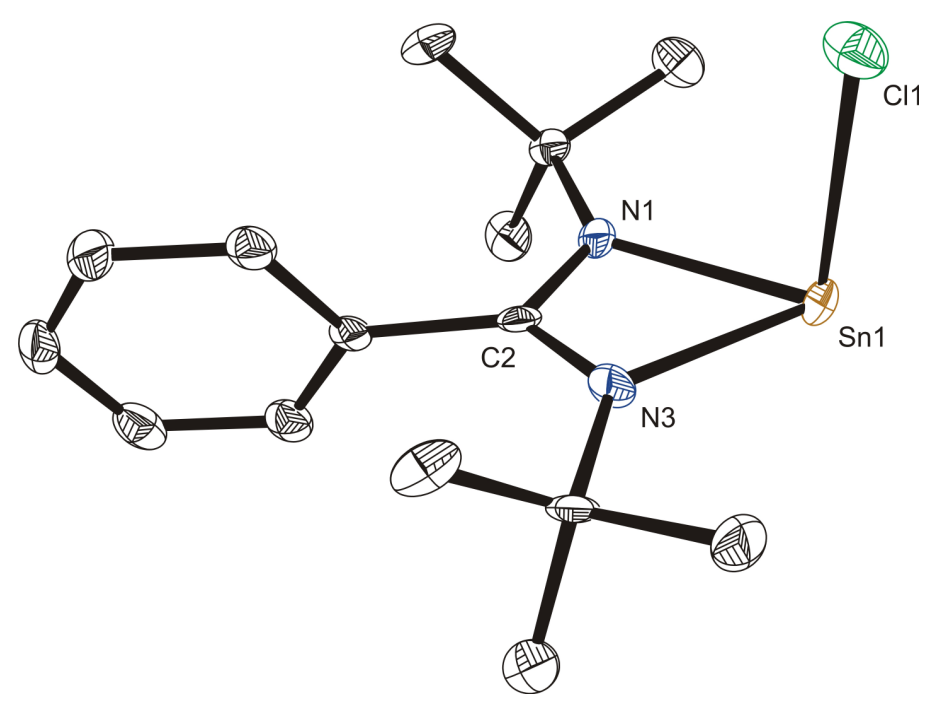

Figure 7.1. X-ray structure of 46. Hydrogen atoms are omitted for clarity. Selected bond distances $(\AA)$ and bond angles (deg): Sn1-N1 2.177(3), Sn1-N3 2.192(3), Sn1-Cl1 2.4831(9); N1-Sn1-N3 60.5(1), N1-Sn1-Cl1 92.28(7), N3-Sn1-Cl1 94.16(8). 
Chapter 7. Synthesis of Monomeric Divalent Tin(II) Compounds with Terminal Chloride, Amide, and Triflate Substituents

Maintaining a toluene solution of $\mathbf{4 6}$ overnight at $-32{ }^{\circ} \mathrm{C}$ resulted in colorless single crystals suitable for X-ray structural analysis. Compound $\mathbf{4 6}$ crystallizes in the triclinic space group $P-1$ (Figure 7.1$).{ }^{44}$ Compared to the similar structures of $\left[\left\{\mathrm{cy}_{2} \mathrm{NC}(\mathrm{NAr})_{2}\right\} \mathrm{SnCl}\right](\mathrm{cy}=$ cyclohexyl), $\quad\left[\left\{\left(\text { cis- } \mathrm{Me}_{2} \mathrm{C}_{5} \mathrm{H}_{8} \mathrm{~N}\right) \mathrm{C}(\mathrm{NAr})_{2}\right\} \mathrm{SnCl}^{130} \text { and }\left[t \mathrm{BuC}(\mathrm{NAr})_{2}\right\} \mathrm{SnCl}\right]^{131} \quad(\mathrm{Ar}=2,6-$ diisopropyl phenyl) the structure of $\mathbf{4 6}$ is very similar showing a distorted pyramidal geometry indicating a stereochemically active lone pair. The bond lengths and angles are in the same range, but the $\mathrm{N}-\mathrm{Sn}-\mathrm{Cl}$ angles $\left(92.3\right.$ and $\left.94.2^{\circ}\right)$ in $\mathbf{4 6}$ have slightly smaller values than in the other structures $\left(94.4\right.$ to $\left.99.8^{\circ}\right)$. The tin atom is $0.20 \AA$ above the plane of the amidinato ligand. In the other three structures this value is smaller ( 0.03 to $0.12 \AA)$.

\subsubsection{Syntheses of $\mathrm{LSnOSO}_{2} \mathrm{CF}_{3}$ and $\mathrm{LSnN}(\mathrm{TMS})_{2}\left(\mathrm{~L}=\mathrm{PhC}(\mathrm{N} t \mathrm{Bu})_{2}\right)$}

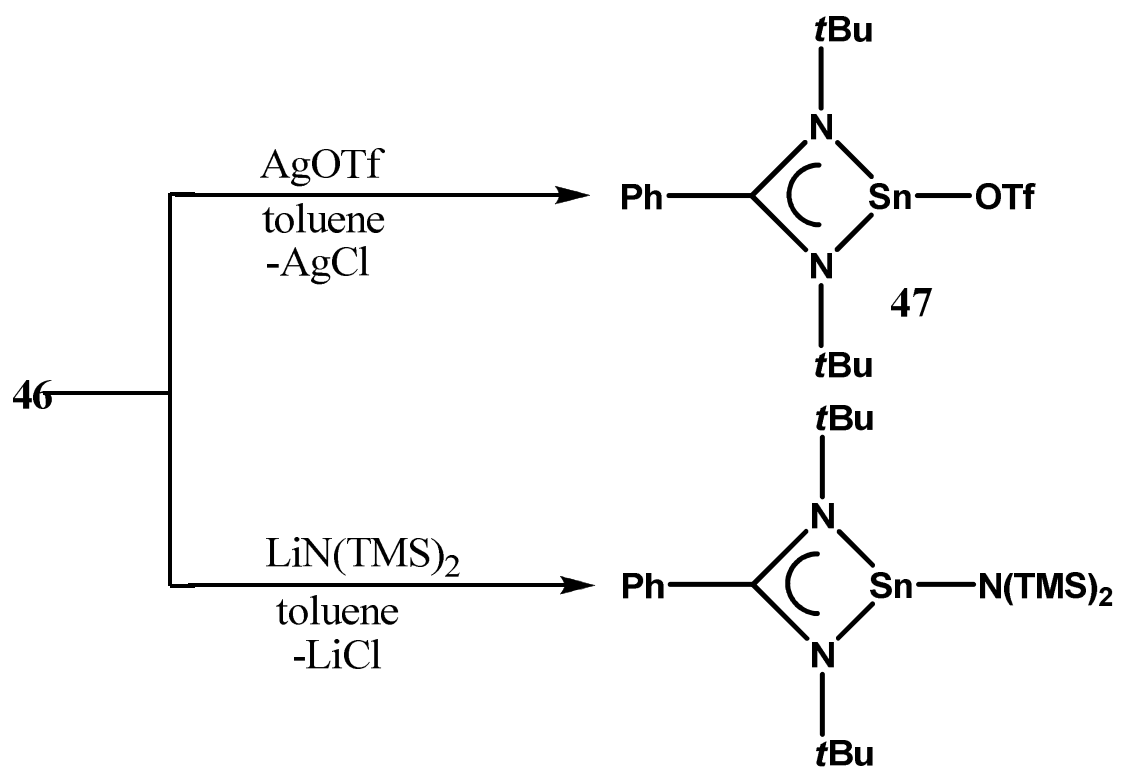

48

Scheme 7.2. Preparation of $\mathbf{4 7}$ and $\mathbf{4 8}$

With the objective of preparing other tin(II) derivatives we tried the substitution reactions of 46 with selected nucleophiles. It is well known that triflate anion $\left(\mathrm{OSO}_{2} \mathrm{CF}_{3}\right)$ has long served as an excellent leaving group in nucleophilic displacement reactions. ${ }^{22}$ Organotin triflates 
Chapter 7. Synthesis of Monomeric Divalent Tin(II) Compounds with Terminal Chloride, Amide, and Triflate Substituents

may act as a precursor for further reactions. Treatment of $\mathbf{4 6}$ with AgOTf in toluene at room temperature for 3 hours afforded the compound $\operatorname{LSnOTf}\left(\mathrm{L}=\operatorname{PhC}(\mathrm{N} t \mathrm{Bu})_{2}\right)$ (47) with good yield $\left(78 \%\right.$ ) (Scheme 7.2). Compound 47 was characterized by ${ }^{1} \mathrm{H},{ }^{13} \mathrm{C},{ }^{19} \mathrm{~F}$, and ${ }^{119} \mathrm{Sn}$ NMR spectroscopy, EI-MS spectrometry and elemental analysis. In the ${ }^{19}$ F NMR 47 exhibits a singlet resonance at $-73 \mathrm{ppm}$ whereas in the ${ }^{119} \mathrm{Sn}$ NMR it resonates at $-33.16 \mathrm{ppm}$. Colorless crystals of $\mathbf{4 7}$ were obtained from a toluene solution at room temperature after 1 day.

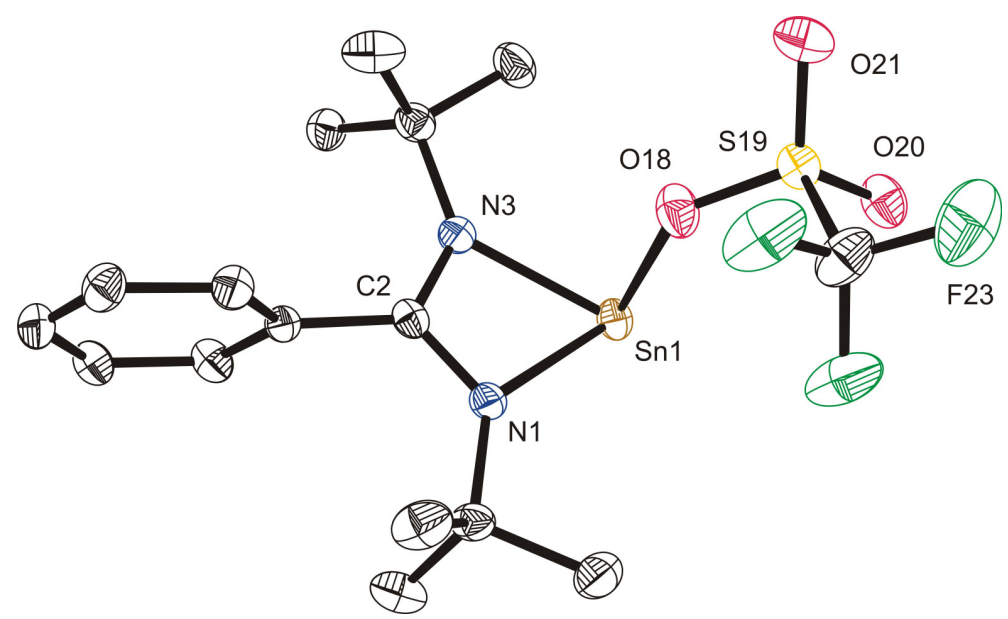

Figure 7.2. X-ray structure of 47. Hydrogen atoms are omitted for clarity. Selected bond distances $(\AA)$ and bond angles (deg): Sn1-N1 2.176(2), Sn1-N3 2.163(2), Sn1-O18 2.362(2), N1-Sn1-N3 60.88(8), N1-Sn1-O18 86.43(8), N3-Sn1-O18 83.09(8).

The structure of $\mathbf{4 7}$ was unequivocally established by X-ray crystallography. ${ }^{44} 47$ crystallizes in the monoclinic space group $P 2_{1} / n$. Important bond lengths and bond angles are given in the legend. The geometry of the amidinato ligand and the coordination of the tin atom are similar to what is found in structure 46. However the $\mathrm{N}-\mathrm{Sn}-\mathrm{O}$ bond angles are much smaller than the $\mathrm{N}-\mathrm{Sn}-\mathrm{Cl}$ angles in 46 (83.1 and 86.5 compared to 92.3 and $\left.94.2^{\circ}\right)$. The distance of the tin atom to the plane of the amidinato ligand is quite similar $(0.23$ compared to $0.20 \AA$ in 46 ). Similar compounds $\quad\left[\mathrm{PhC}\left(\mathrm{NSiMe}_{3}\right)_{2} \mathrm{SnOCPh}_{3}\right], \quad\left[\mathrm{PhC}\left(\mathrm{NSiMe}_{2} \mathrm{Ph}\right)_{2} \mathrm{SnOCPh}_{3}\right]^{132}$, $\left[t \mathrm{BuC}(\mathrm{NAr})_{2} \mathrm{SnO} i \mathrm{Pr}\right]^{131}(\mathrm{Ar}=2,6$-diisopropyl phenyl) show smaller Sn-O bond lengths $(2.006$ 
Chapter 7. Synthesis of Monomeric Divalent Tin(II) Compounds with Terminal Chloride, Amide, and Triflate Substituents

to $2.040 \AA$ compared to $2.362 \AA$ ) while the $\mathrm{Sn}-\mathrm{N}$ bonds are longer $(2.208$ to $2.252 \AA$ compared to 2.163 and $2.176 \AA$ ) and the $\mathrm{N}-\mathrm{Sn}-\mathrm{O}$ angles wider (87.3 to $94.8^{\circ}$ compared to 83.1 and $\left.86.5^{\circ}\right)$

Our interest in subvalent group 14 metal bis(trimethylsilyl) amides derives from work with the isoelectronic bis(trimethylsilyl) methyl derivatives and on earlier studies on many amides, including $\mathrm{SnMe}_{3}\left(\mathrm{NMe}_{2}\right){ }^{133}$ The bis(trimethylsilyl) amido ligand was chosen because a) its size often stabilizes complexes in which the metal has a low coordination number, b) the numerous methyl groups provide for good hydrocarbon solubility. Compound LSnN(TMS $)_{2}$ (48) was obtained in high yield from the reaction of $\mathbf{4 6}$ with 1 equivalent of $\operatorname{LiN}(\mathrm{TMS})_{2}$ at room temperature in diethyl ether (Scheme 7.2). Compound $\mathbf{4 8}$ is a white solid soluble in benzene, THF, toluene and shows no decomposition on exposure to air for a short period of time. The amidinato tin amide is a very interesting compound because it can act as a precursor for polymerization and hydrolysis reactions. Richeson and his team showed that amidinato tin(II) amide compounds are good catalysts for cyclotrimerization of phenyl isocyanate to triphenyl isocyanurates. ${ }^{133 \mathrm{~d},}$, 133e 48 was characterized by ${ }^{1} \mathrm{H},{ }^{13} \mathrm{C},{ }^{29} \mathrm{Si}$, and ${ }^{119} \mathrm{Sn} \mathrm{NMR}$ spectroscopy, EI mass spectrometry, elemental, and X-ray structural analysis. The ${ }^{1} \mathrm{H}$ NMR spectrum of compound 48 shows two singlets at (1.07 ppm and $0.25 \mathrm{ppm})$ corresponding to $t \mathrm{Bu}$ protons and $\mathrm{SiMe}_{3}$ protons respectively, and one multiplet $(7.34-7.39 \mathrm{ppm})$ for the $\mathrm{Ph}$ protons. The ${ }^{119} \mathrm{Sn}$ NMR of 48 exhibits a singlet at $112 \mathrm{ppm}$. The ${ }^{29} \mathrm{Si}$ NMR shows a resonance at $5.26 \mathrm{ppm}$. The molecular ion peak is observed with the highest relative intensity in the EI-MS spectrum at $m / z 511$.

An X-ray diffraction study on single crystal of $\mathbf{4 8}$ obtained from a toluene solution kept at -30 ${ }^{\circ} \mathrm{C}$ in a freezer for 1 day confirmed the features deduced from the spectroscopic data. Compound 48 crystallizes as a pseudo-merohedral twin (twin fraction $0.473(2)$ ) in the 
Chapter 7. Synthesis of Monomeric Divalent Tin(II) Compounds with Terminal Chloride, Amide, and Triflate Substituents

triclinic space group $P-1$ with two very similar molecules in the asymmetric unit. We find the typical geometry as in $\mathbf{4 6}$ and $\mathbf{4 7}$. But here the mean bond lengths between tin and the amidinate nitrogen atoms $(2.21 \AA)$ are longer than the average value in 46 and 47 (2.18 and 2.17 $\AA$ ). But the value corresponds to values found in similar structures: $\left[t \mathrm{BuC}\left(\mathrm{NSiMe}_{3}\right)_{2} \mathrm{SnN}\left(\mathrm{SiMe}_{3}\right)_{2}\right]^{133 \mathrm{~d}}, \quad\left[t \mathrm{BuC}(\mathrm{Ncyclohexyl})_{2} \mathrm{SnN}\left(\mathrm{SiMe}_{3}\right)_{2}\right]^{133 \mathrm{e}} \quad$ and $\left[t \mathrm{BuC}(\mathrm{NAr})_{2} \mathrm{SnNMe}_{2}\right]^{131 \mathrm{~d}}(\mathrm{Ar}=2,6$-diisopropyl phenyl) $(2.19$ to $2.26 \AA)$ as well as the mean value for the $\mathrm{Sn}-\mathrm{N}$ (from monodentate amide) bond length $(2.13 \AA$ in $\mathbf{4 8})$ compared to the two other $\mathrm{LSnNSiMe}_{3}$ structures (2.12 and $2.14 \AA$ ) and the N-Sn-NSiMe 3 bond angles (102.1 to $106.4^{\circ}$ in $\mathbf{4 8}$ compared to 99.0 to $105.4^{\circ}$ ). In these structures the deviation of the tin atom from the plane of the amidinato ligand is larger ( 0.5 in $\mathbf{4 8}$ and 0.3 to $0.5 \AA$ in the others) compared to 46 and 48.

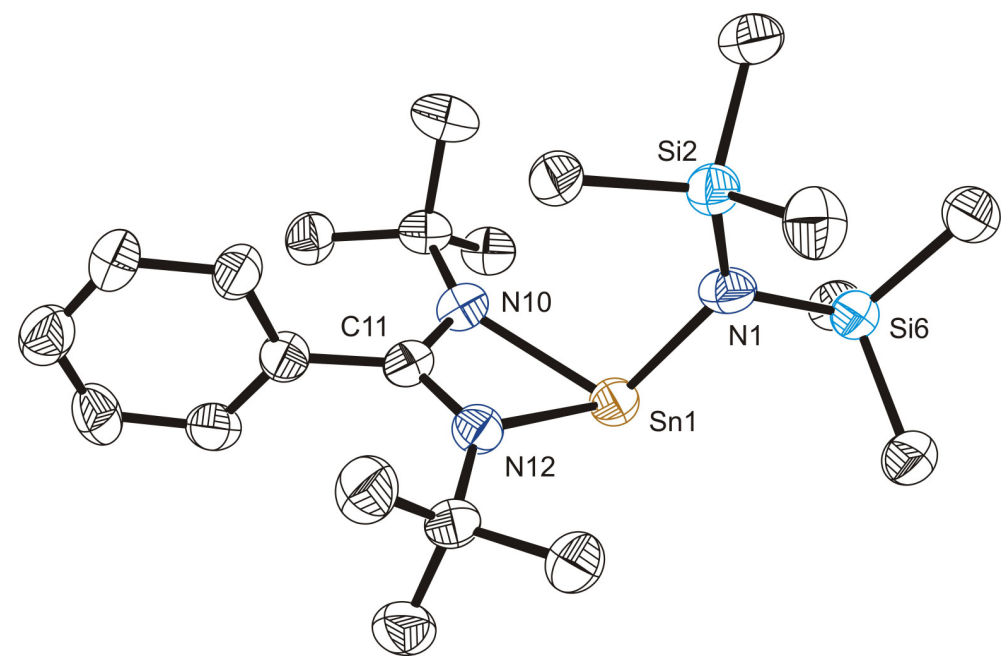

Figure 7.3. X-ray structure of 48. There are two molecules in the asymmetric unit. Molecule 1 is shown and hydrogen atoms are omitted for clarity. Selected bond distances $(\AA)$ and bond angles (deg) for molecule 1: Sn1-N1 2.116(6), Sn1-N10 2.216(6), Sn1-N12 2.227(6), N1-Sn1-N10 103.6(2), N1-Sn1-N12 105.0(2), N10-Sn1-N12 59.9(2). Selected bond distances $(\AA)$ and bond angles (deg) for molecule 2 (not shown): Sn2-N1' 2.134(5), Sn2-N10' 2.191(7), Sn2-N12' 2.214(6), N1'-Sn2-N10' 102.1(3), N1'-Sn2-N12' 106.4(2), N10'-Sn2-N12' 59.3(2.). 


\subsubsection{Synthesis of $\left[\mathrm{PhC}(\mathrm{N} t \mathrm{Bu})_{2}\right]_{2} \mathrm{Sn}$}

Organotin(II) hydrides generally can be prepared by the reduction of the corresponding chlorides with $\mathrm{AlH}_{3} \cdot \mathrm{NMe}_{3} \cdot{ }^{134}$ However treatment of $\mathbf{4 6}$ with $\mathrm{AlH}_{3} \cdot \mathrm{NMe}_{3}$ in toluene at room temperature does not lead to LSnH. However, recently we successfully synthesized LSnH $\left(\mathrm{L}=\mathrm{HC}(\mathrm{CMeNAr})_{2},\left(\mathrm{Ar}=2,6-i-\mathrm{Pr}_{2} \mathrm{C}_{6} \mathrm{H}_{3}\right)\right.$ in good yield and without any impurity with $\mathrm{K}\left[\mathrm{HB}(\mathrm{sec}-\mathrm{Bu})_{3}\right]$ (commonly known as K-selectride) reagent. ${ }^{135}$ Consequently compound 46 was treated with 1 equivalent of L-selectride and this resulted in the formation of $\mathrm{L}_{2} \mathrm{Sn}(49)$. The propensity of the formation of homoleptic tin compound is due to the instability of the amidinato tin(II) hydride compound. 49 was characterized by ${ }^{1} \mathrm{H},{ }^{13} \mathrm{C},{ }^{119} \mathrm{Sn} \mathrm{NMR}$ spectroscopy, EI-MS spectrometry, elemental, and X-ray structural analysis. The ${ }^{1} \mathrm{H}$ NMR spectrum exhibits a singlet at $1.12 \mathrm{ppm}$, which was attributed to 36 protons of $t \mathrm{Bu}$ group, and a multiplet at $7.30-7.34 \mathrm{ppm}$ which is attributed to 10 protons. The ${ }^{119} \mathrm{Sn}$ NMR of 49 shows a resonance at $-285 \mathrm{ppm}$, which is upfield shifted, when compared with that of $\mathbf{1}$. In the EI-MS spectrum only smaller ions are observed. Further attempts to prepare the $\mathrm{Sn}-\mathrm{H}$ compound by $\mathrm{NaBH}_{4}, \mathrm{KH}$, and $\mathrm{NaH}$ respectively failed.

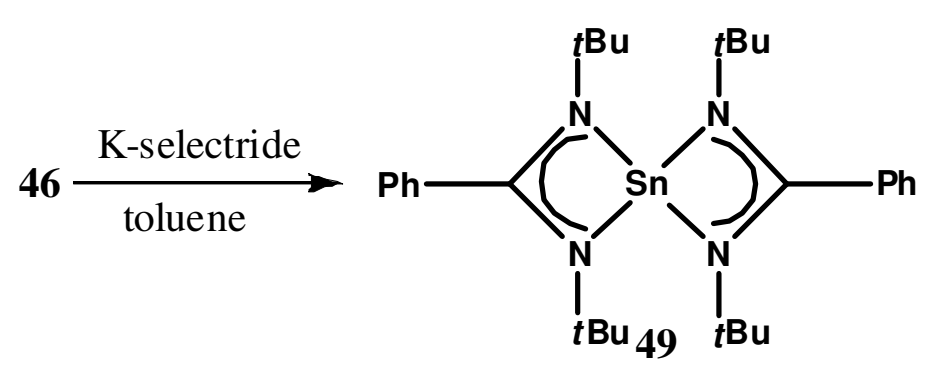

Scheme 7.3. Preparation of 49 


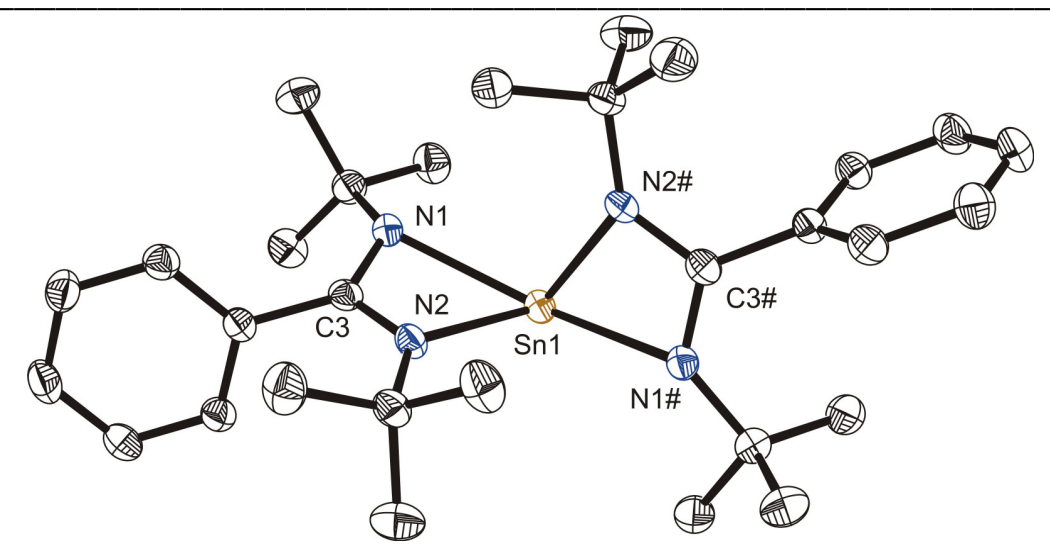

Figure 7.4. X-ray structure of 49. Hydrogen atoms are omitted for clarity. Symmetry-related atoms are denoted with \#. Selected bond distances $(\AA)$ and bond angles (deg) Sn1-N2 2.195(1), Sn1-N1 2.394(1), N2-Sn1-N2\# 98.12(8) N2-Sn1-N1 57.75(5) N2\#-Sn1N1 103.97(5), N1-Sn1-N1\# 154.24(7).

Maintaining a toluene solution of 49 at $-30{ }^{\circ} \mathrm{C}$ in a freezer afforded temperature sensitive colorless crystals suitable for X-ray analysis. Compound 49 crystallizes as a non-merohedral twin (twin fraction $0.0252(9)$ ) in the monoclinic space group $C 2 / c$ with half a molecule and a solvent toluene molecule in the asymmetric unit. The coordination geometry around $\mathrm{Sn} 1 \mathrm{can}$ be viewed as distorted saw-horse like, with N1 and N1\# in the axial positions and N2 and N2\# residing in the equatorial plane. Accordingly the axial Sn- N(1) bond distance is longer (2.394(1) $\AA$ ) than the Sn-N(2) bond length with 2.195(1) $\AA$. There are several known structures with two coordinating amidinato ligands to $\operatorname{Sn}(\mathrm{II}),{ }^{[26]}$ e.g. $\left[\left(\mathrm{PhCNSiMe}_{3} \mathrm{~N} t \mathrm{Bu}\right)_{2} \mathrm{Sn}^{136}\right.$ All have a more or less distorted saw-horse like coordination with two longer $(2.32-2.44 \AA)$ and two shorter bonds $(2.15-2.27 \AA)$, but the "axial" angle varies (119.6 to $148.8^{\circ}$ ) showing the highest value in 49. Accordingly also the angle between the planes of the amidinato ligands varies $\left(42.4\right.$ to $89.3^{\circ}, 53.8^{\circ}$ in 49$)$.

\subsubsection{Reaction of $\left[\mathrm{PhC}(\mathrm{N} t \mathrm{Bu})_{2}\right] \mathrm{SnCl}$ with $\mathrm{Fe}_{2}(\mathrm{CO})_{9}$}

The aptitude of silylene, germylene, and stannylene to act as a $\sigma$-donor, and $\pi$-acceptors already established them as preeminent ligands for the synthesis of transition metal complexes with potential application in homogeneous catalysis. ${ }^{67,68,69}$ After the successful isolation of 
Chapter 7. Synthesis of Monomeric Divalent Tin(II) Compounds with Terminal Chloride, Amide, and Triflate Substituents

chlorostannylene we have been intrigued by the question whether such tri-coordinate stannylenes can act as ligands to stabilize metal complexes. Therefore, $\left[\mathrm{PhC}(\mathrm{N} t \mathrm{Bu})_{2}\right] \mathrm{SnCl}$ and diiron nonacarbonyl $\left[\left(\mathrm{Fe}_{2}(\mathrm{CO})_{9}\right]\right.$ were selected as a probe to investigate their reaction behavior. The synthetic procedure of $\mathbf{5 0}$ is similar to that of $\left\{\left[\mathrm{PhC}(\mathrm{N} t \mathrm{Bu})_{2}\right] \mathrm{SiO} t \mathrm{Bu}\right\} \mathrm{Fe}(\mathrm{CO})_{4}{ }^{69}$

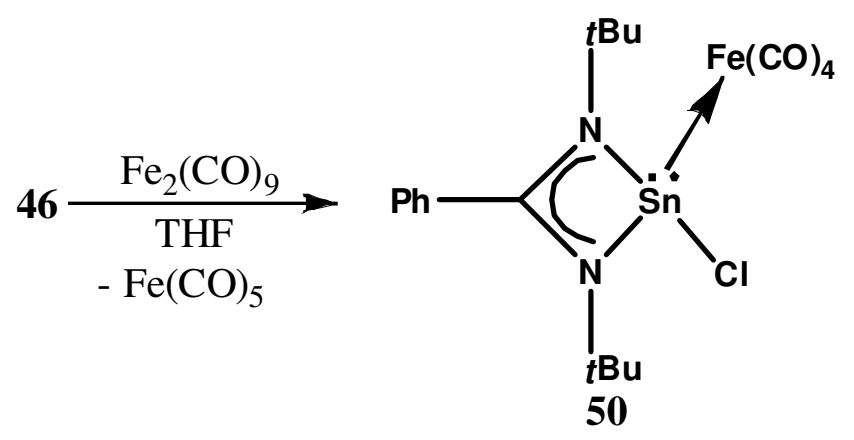

Scheme 7.4. Preparation of 50.

Treatment of $\mathbf{4 6}$ with 1.2 equivalent of diiron nonacarbonyl in THF for $40 \mathrm{~h}$ afforded complex 50. The solvent was then removed in vacuum, and the residue was extracted with toluene. The insoluble solid was filtered off, and the filtrate was concentrated to yield a red-brown solid. 50 was characterised by NMR spectroscopy, EI-MS spectrometry, and elemental analysis. In the ${ }^{119} \mathrm{Sn}$ NMR $\mathbf{5 0}$ exhibits a sharp resonance at $255 \mathrm{ppm}$, which indicates the coordination of the Fe atom to tin which resulted in a downfield chemical shift in the ${ }^{119} \mathrm{Sn}$ NMR spectrum $\left({ }^{119} \mathrm{Sn}\right.$ of 46: $\left.29.6 \mathrm{ppm}\right)$. This downfield chemical shift suggests the removal of electron density from tin atom upon product formation. Similar kind of downfield chemical shift was observed for the $t \mathrm{Bu}$ protons of the amidinato ligand, which resonates at $1.41 \mathrm{ppm}$, when compared to that of $\mathbf{4 6}(\delta 1.08 \mathrm{ppm})$. In the EI-MS spectrum the molecular ion appeared as the most abundant peak with highest relative intensity at $\mathrm{m} / \mathrm{z}$ 553. All data corroborate the formation of 50. Here it is worth to point out that $\mathbf{5 0}$ can be an efficient precursor for the synthesis of amidinato stabilized tin(II) hydroxide as very recently we have shown that 
Chapter 7. Synthesis of Monomeric Divalent Tin(II) Compounds with Terminal Chloride, Amide, and Triflate Substituents

reaction of $\mathrm{L}_{1} \mathrm{Sn}\left(\mathrm{NMe}_{2}\right) \mathrm{Fe}(\mathrm{CO})_{4}\left(\mathrm{~L}_{1}=\mathrm{CH}\left\{(\mathrm{CMe})\left(2,6-i \mathrm{Pr}_{2} \mathrm{C}_{6} \mathrm{H}_{3} \mathrm{~N}\right)\right\}_{2}\right)$ with $\mathrm{H}_{2} \mathrm{O}$ gave the first example of a monomeric tin(II) hydroxide complex $\mathrm{L}_{1} \mathrm{Sn}(\mathrm{OH}) \mathrm{Fe}(\mathrm{CO})_{4 \cdot}{ }^{137}$

\subsection{Conclusion}

In conclusion we have prepared the monomeric tin(II) chloride stabilized by bulky amidinato ligand. By taking advantage of nucleophilic substitution reactions using AgOTf, and LiN(TMS $)_{2}$ respectively we synthesized the divalent tin(II) monomer with different substituents. These compounds can act as a good precursor for catalysis and polymerization reactions. 


\section{Chapter 8}

\subsection{Summary}

In this thesis we highlighted the synthesis of alkine analogue of heavier group 14 elements and investigated their unprecedented reactivity towards small molecules. The initial task was to gain an appreciation of the affinity of $\mathrm{Si}, \mathrm{Ge}$, and $\mathrm{Sn}$ with sterically demanding benzamidinato ligand with $t \mathrm{Bu}$ substituents on the nitrogen atoms because the choice of ligand is very crucial in organometallic chemistry. With the support of this ligand we were able to synthesize and structurally characterize a bis-silylene $\left[\mathrm{PhC}(\mathrm{N} t \mathrm{Bu})_{2}\right]_{2} \mathrm{Si}_{2}(\mathbf{3 5})$. The compound was prepared by the simple expedient of reacting $\mathrm{SiCl}_{4}$ with $\mathrm{Li}[\mathrm{Bu} t \mathrm{NC}(\mathrm{Ph}) \mathrm{NBu} t]$ and treating the resulting $\mathrm{LSiCl}_{3}\left(\mathrm{~L}=\mathrm{PhC}(\mathrm{N} t \mathrm{Bu})_{2}\right)$ with potassium graphite. It displays a unique structure in which two silicon atoms are connected by a single bond and each silicon atom is having a lone pair of electrons. To elucidate the electronic structure and bonding characteristics of $\mathbf{3 5}$, theoretical calculation was done. NAO and NBO analyses of $\mathbf{3 5}$ show that the two Si centers are connected by a $\sigma$ bond, characterized by the Wiberg bond order of 0.9768 with high $p$ character $(s: 18.27 \%, p: 81.73 \%)$. Interestingly, the single non-bonded electron pair on each silicon atom is found to reside in an orbital with very high $s$-character $(s: \sim 65 \%, p: \sim 35 \%)$. The preparation of $\mathbf{3 5}$ was followed by the synthesis of its germanium version $\left[\mathrm{PhC}(\mathrm{N} t \mathrm{Bu})_{2}\right]_{2} \mathrm{Ge}_{2}$ (42). It must be noted that both 35 and 42 are red-orange crystalline substance with truly astonishing thermal stability and persistent at room temperature under an inert atmosphere. Another important aspect is their geometry. All the heavier group 14 alkine analogues reported so far possess trans-bent geometry, but these are the first two alkine analogues with gauche-bent geometry. The $\mathrm{Si}-\mathrm{Si}$ bond in $\mathbf{3 5}$ is $2.41 \AA$, whereas the Ge-Ge bond in 42 is $2.57 \AA$, which corresponds to the single bond character. The designation of the metal environments in $\mathbf{3 5}$ and $\mathbf{4 2}$ is distorted tetrahedral. Moreover, calculations based upon the structure suggest a gauche conformation arising from the $\mathrm{Si}-\mathrm{Si}$ and $\mathrm{Ge}-\mathrm{Ge}$ bonds 
analogous to hydrazine. The formation of $\mathrm{Si}-\mathrm{Si}$ and $\mathrm{Ge}-\mathrm{Ge}$ bond with the support of amidinato ligand showed that the classical $\sigma / \pi$ model of the double bond in carbon, nitrogen, and oxygen compounds is not necessarily applicable to the heavier elements. It also must be noted that a similar type bis-carbene is not reported so far.

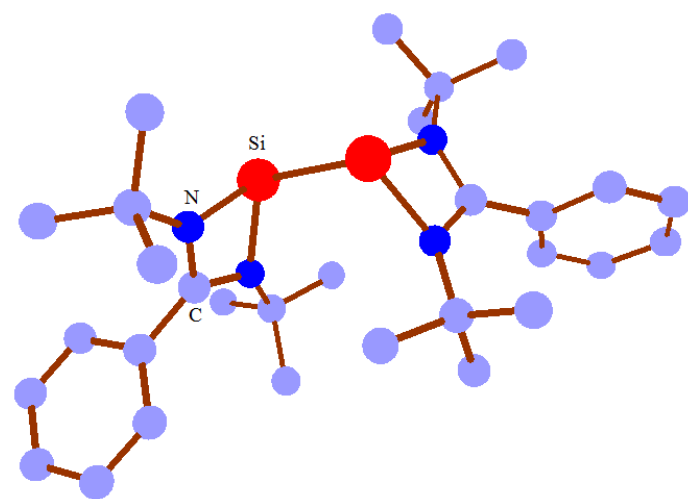

35

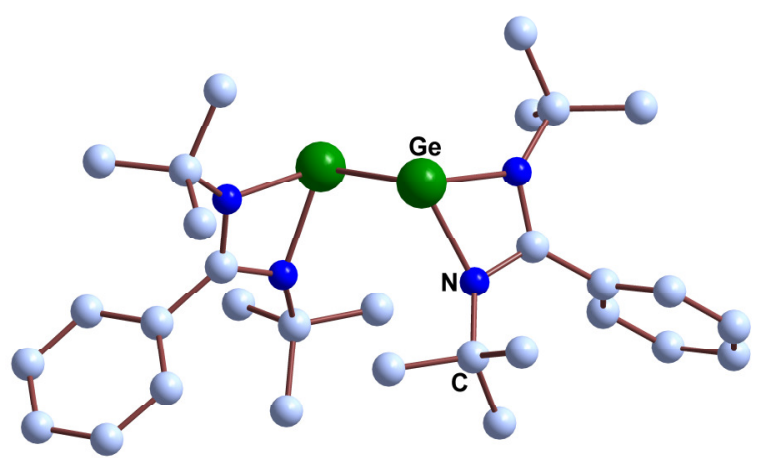

42

The success in isolating interconnected silylene and germylene prompted us to study its reactivity, which is of fundamental importance to ascertain the hallmark of this special class of compounds. We reacted 35 with ketone, diketone, $\mathrm{N}_{2} \mathrm{O}$, white phosphorus. In the reaction with ketone and $\mathrm{N}_{2} \mathrm{O}$ the central $\mathrm{Si}-\mathrm{Si}$ bond was cleaved and led to the formation of a $\mathrm{Si}_{2} \mathrm{O}_{2}$ four -membered ring (36) and two four -membered $\mathrm{Si}_{2} \mathrm{O}_{2}$ rings connected with two oxygen atoms (38), respectively. In sharp contrast, when $\mathbf{3 5}$ was reacted with benzil, it afforded the formation of bis(siladioxolene) (37) with the preservation of the Si-Si bond. Treatment of white phosphorus with $\mathbf{3 5}$ resulted in the formation of a new cyclobutadiene (CBD) analogue with four heteroatoms (39). All the compounds are characterized by spectroscopic tools and single crystal X-ray diffraction. 


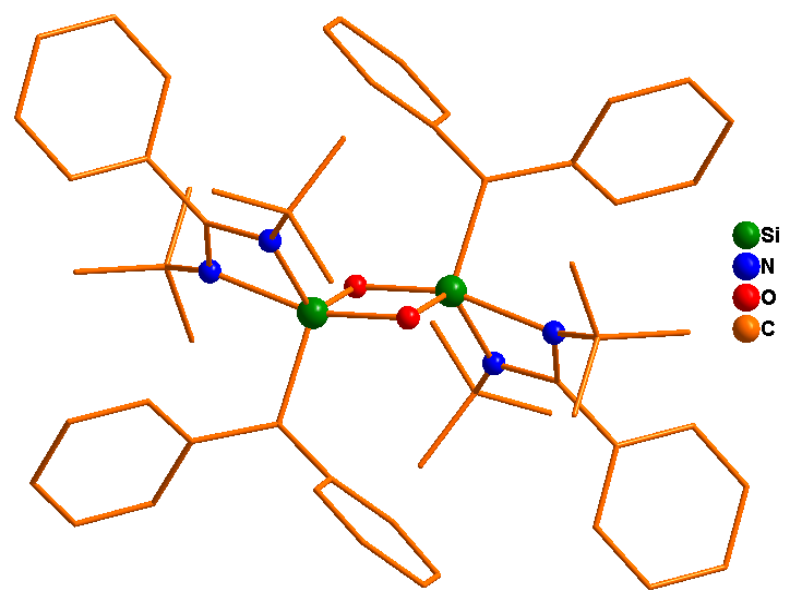

36

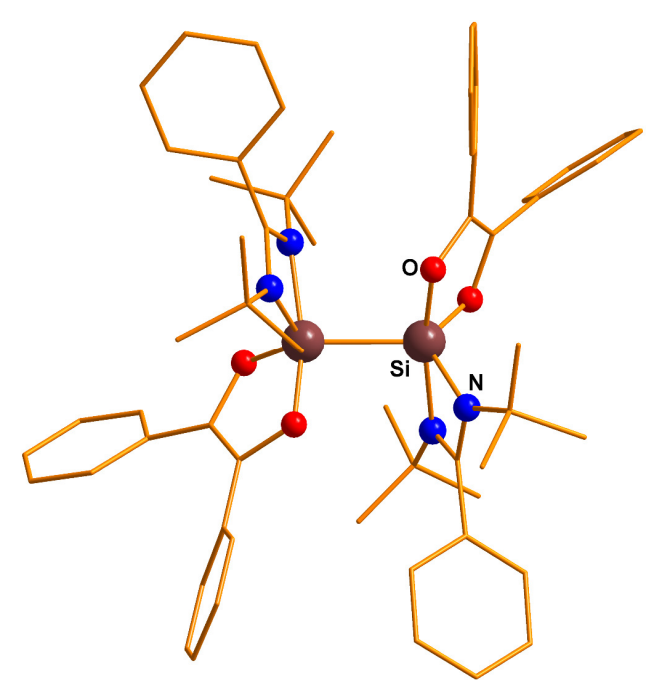

37

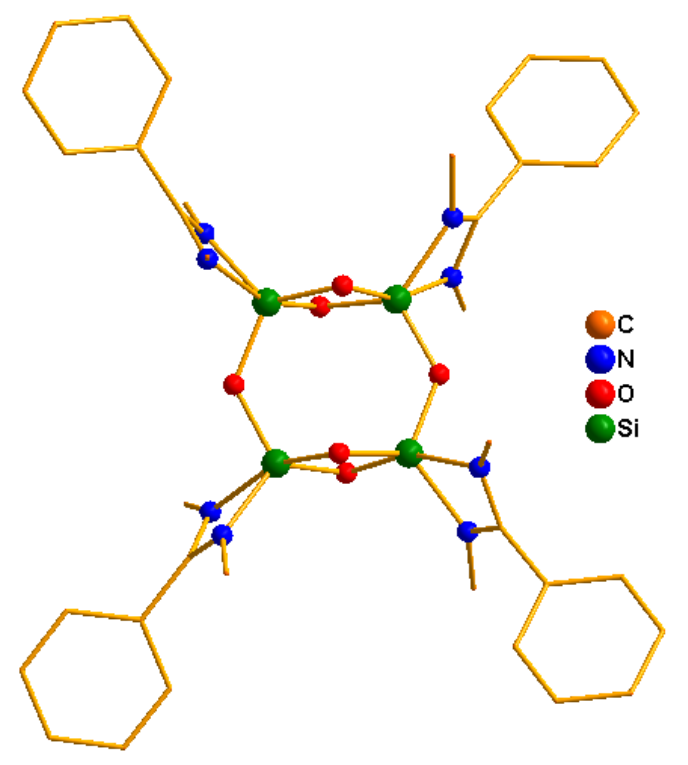

38

Treatment of white phosphorus with $\mathbf{3 5}$ resulted in the formation of a new cyclobutadiene analogue with four heteroatoms (39). This is the first analogue of cyclobutadiene, where all four carbon atoms are replaced by heteroatoms. All four Si-P bond lengths are the same with $2.18 \AA$, which is in between the single and double bond. The two P-Si-P bond angles are exactly the same $\left(107.5(3)^{\circ}\right)$. All these data indicate an extensive delocalization of the $4 \pi$ 
electrons over a four -center bonding molecular orbital comprised of the $\mathrm{p}_{\mathrm{z}}$-atomic orbital of silicon and phosphorus.

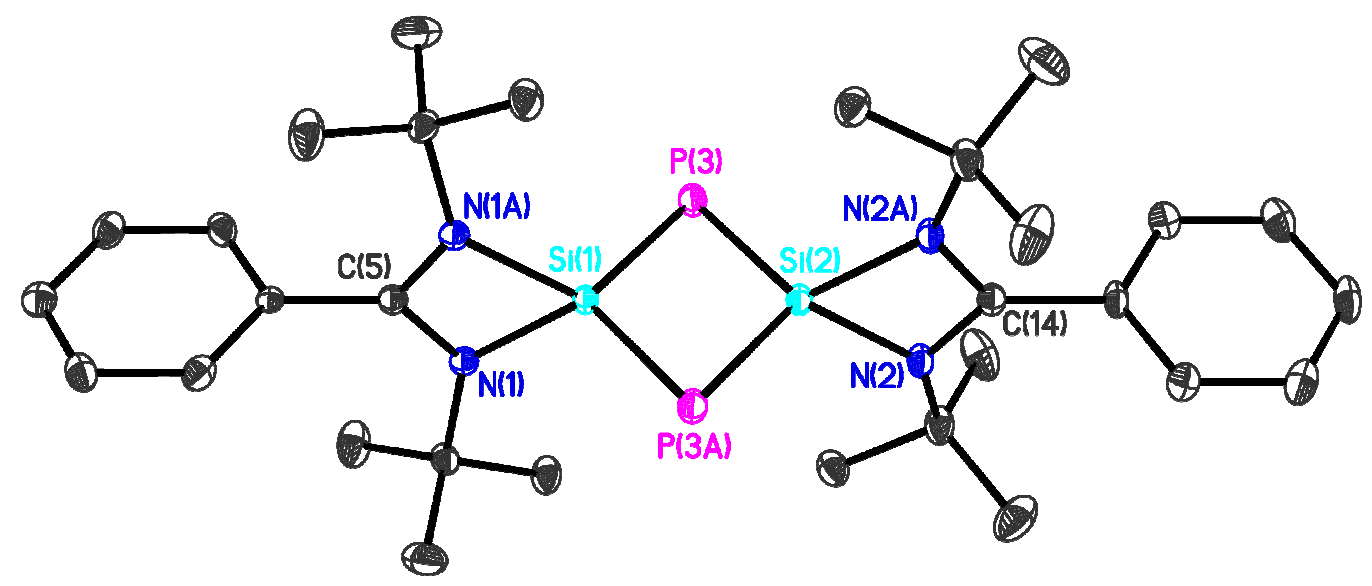

39

When 35 is reacted with biphenyl alkine in 1:2 molar ratio, it leads to the formation of 1,4disilabenzene (40). The four Si-C bonds in $\mathbf{4 0}$ are of $1.800(3) \AA$, which are shorter than the Si-C single bond and longer than that of the Si-C double bond found in literature. ${ }^{12,59}$ NICS (nuclear independent chemical shift) calculation showed that the compound has some aromatic character (-3.64). This is the first example of a room temperature stable of 1,4disilabenzene which was only observed before by UV-Vis spectroscopy in a frozen Ar matrix at $10 \mathrm{~K}$ and by chemical trapping of the intermediate 1,4-disilabenzenes with $\mathrm{MeOH}$ or alkine derivatives. 


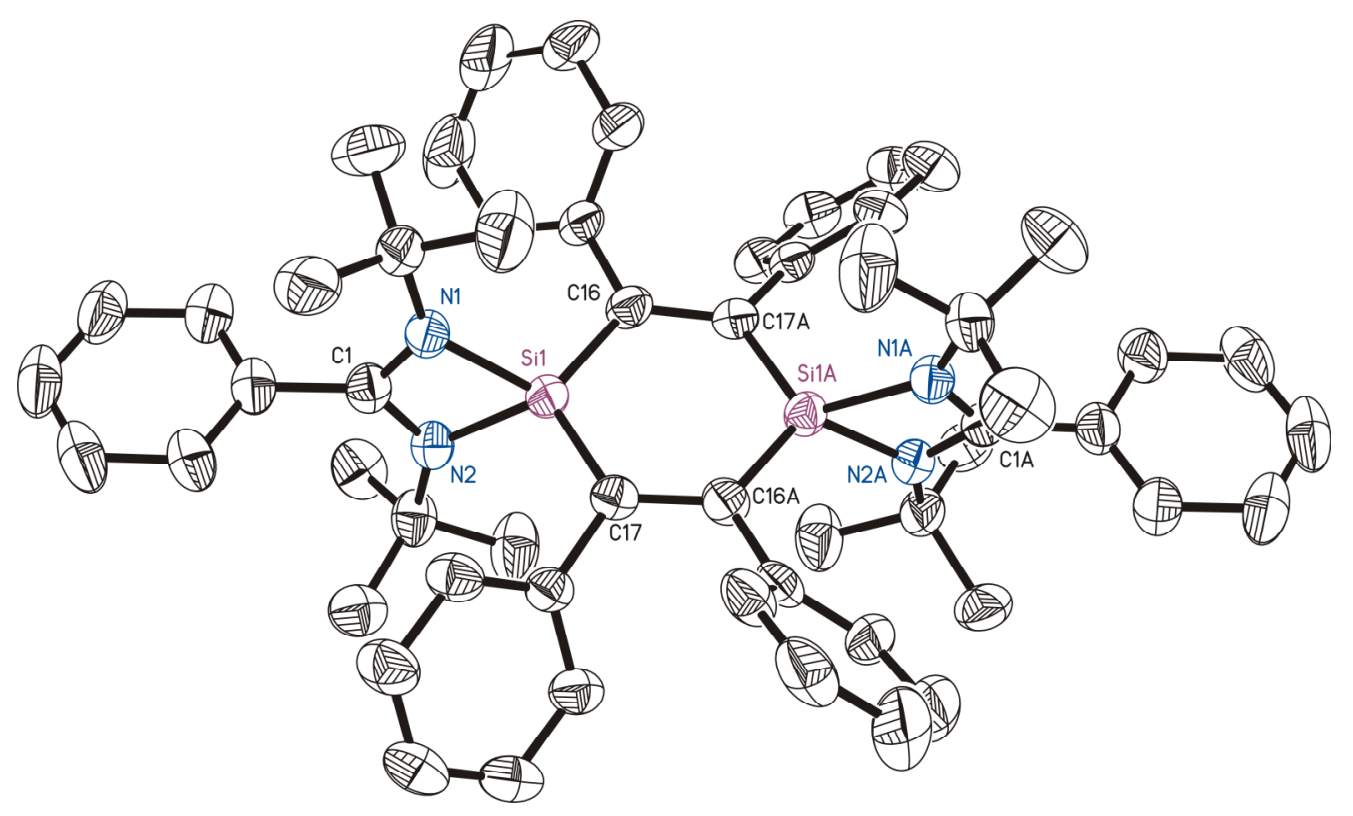

40

To explore the chemistry of bis-germylene (42) we carried out reactions with diazobenzene and diironnonacarbonyl. In the former case the cleavage of the $\mathrm{Ge}-\mathrm{Ge}$ bond occurs to form a vicinal digermahydrazine derivative (44), whereas in the latter case a Lewis acid-base adduct (45) was formed keeping the Ge-Ge bond intact. The most interesting feature of $\mathbf{4 4}$ is that it is a molecular chain containing four elements each with a lone pair of electrons, whereas $\mathbf{4 5}$ is the first example where the $\mathrm{Fe}(\mathrm{CO})_{4}$ moiety is attached to a germanium atom in the formal oxidation state of $+\mathrm{I}$.

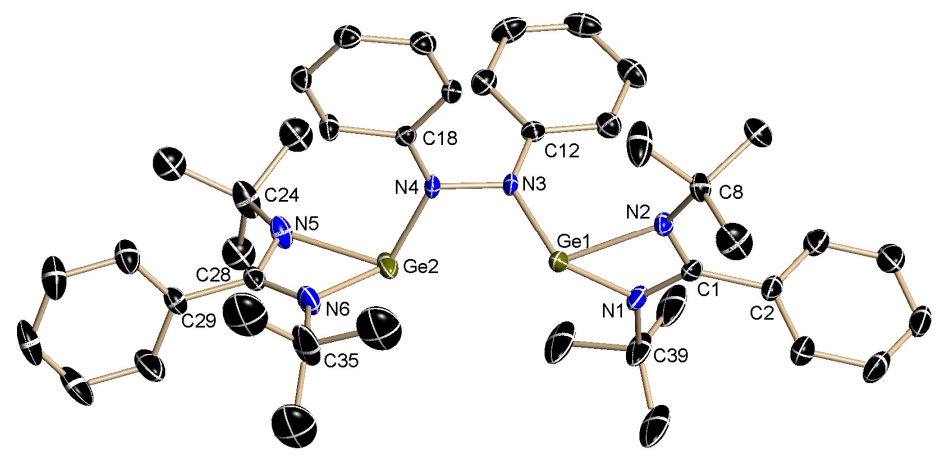

44

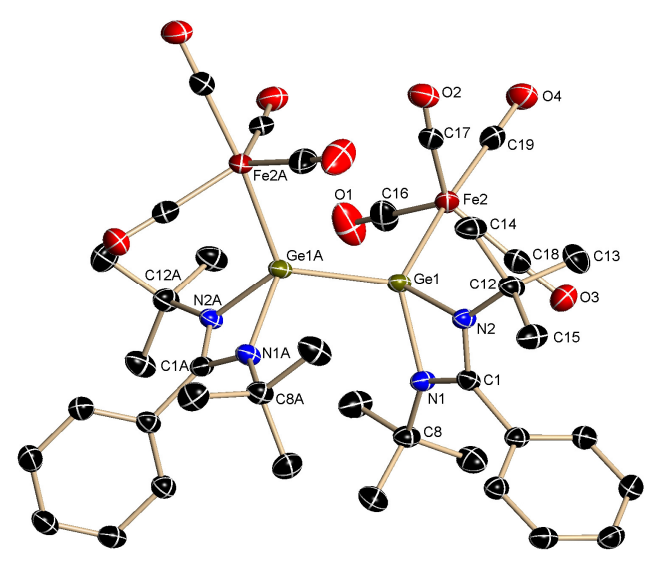

45 
In 2006, our group reported the synthesis of the first heteroleptic chloro silylene $\mathrm{PhC}(\mathrm{N} t \mathrm{Bu})_{2} \mathrm{SiCl}(\mathbf{1 8})$, but the yield of the reaction is only $10 \%$. The paucity of yield hindered our further investigation but we found out a very convenient and facile route to access $\mathbf{1 8}$. Treatment of $\mathrm{PhC}(\mathrm{N} t \mathrm{Bu})_{2} \mathrm{SiHCl}_{2}(\mathbf{2 1})$ with $\mathrm{LiN}(\mathrm{TMS})_{2}$ leads to the formation of $\mathbf{1 8}$ in $90 \%$ yield.<smiles>CN(C[Si]1(Cl)N(C(C)(C)C)C(c2ccccc2)N1C(C)(C)C)C1=NN(C(C)(C)C)[Si]1Cl</smiles>

The increase in yield helped us to study the reactivity of 18. Silicon-containing small ring compounds are one of the most wonderful edifices in modern organosilicon chemistry due to their very unusual structural properties and high reactivity. Synthesis and characterization of silicon containing ring compounds is of fundamental interest and synthetic challenge. Accordingly, we reacted $\mathbf{1 8}$ with biphenyl alkine, ketone, diketone, tert-butyl isocyanate, carbodiimide, phosphalkine etc. With biphenyl alkine it afforded 1,2-disilacyclobutadiene (22), whereas with ketone and diketone it resulted in the formation of monosilaepoxide (24), and monosilaoxolene (25). The formation of stable silaoxirane by the reaction of silylene and ketone proves that base stabilization allows isolation of silaoxirane which are otherwise assumed to exist as reactive intermediate. With carbodiimide it yielded a silimine complex (26) with $\mathrm{Si}=\mathrm{N}$ double bond whereas with tert-butyl isocyanate it led to the formation of a $\mathrm{Si}_{2} \mathrm{O}_{2}$ core (29). The most intriguing reaction happened with adamantylphosphaalkine, which led to the formation of a 1,3-disilacarbaphosphide (32), an analogue of a stable 
cyclobutadiene containing three heteroatoms. The $\mathrm{Si}-\mathrm{C}$ bond lengths in $\mathbf{3 2}$ are exactly identical, while the same is true for Si-P bonds. The interatomic Si...Si distance $(2.48 \AA)$ inevitably proved that there is no $\mathrm{Si}-\mathrm{Si}$ bond in 32. All compounds were characterized by single crystal X-ray diffraction, multinuclear NMR spectroscopy, EI-MS spectrometry and elemental analysis.

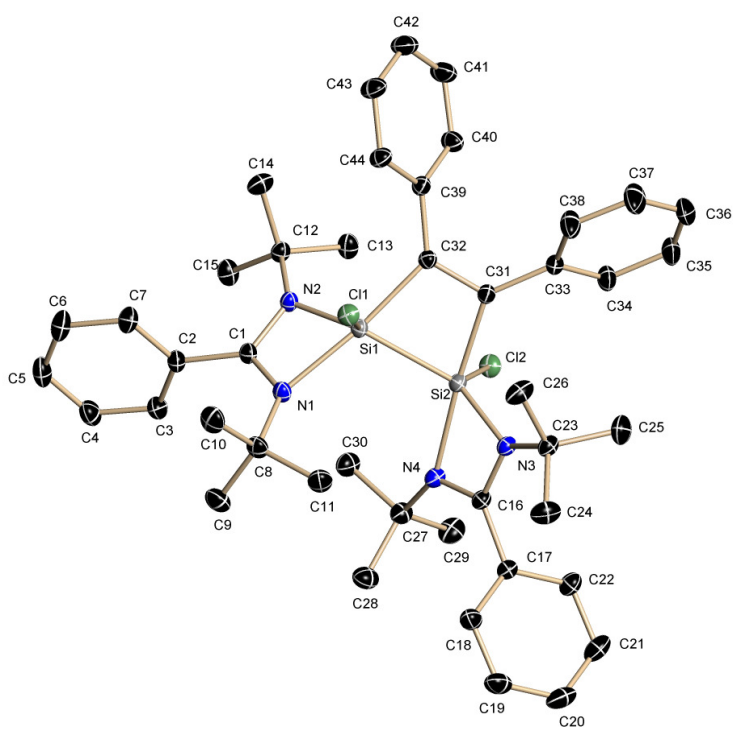

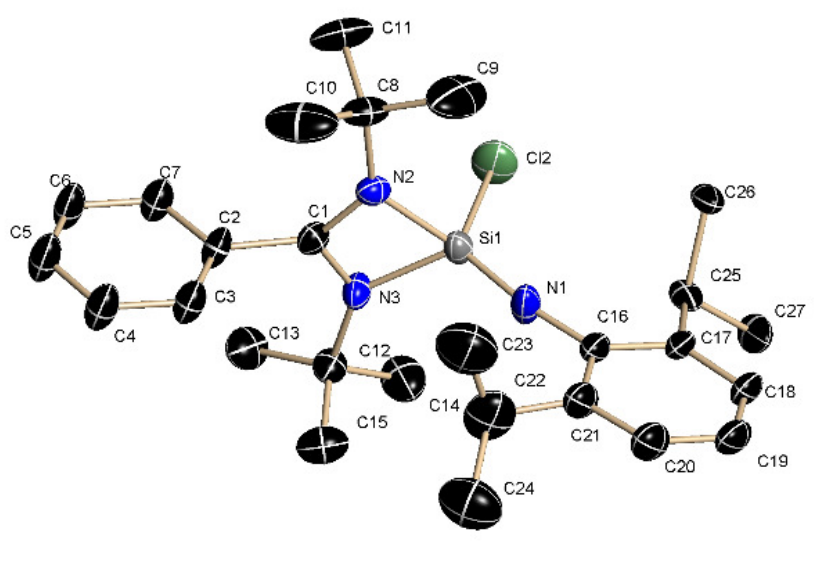
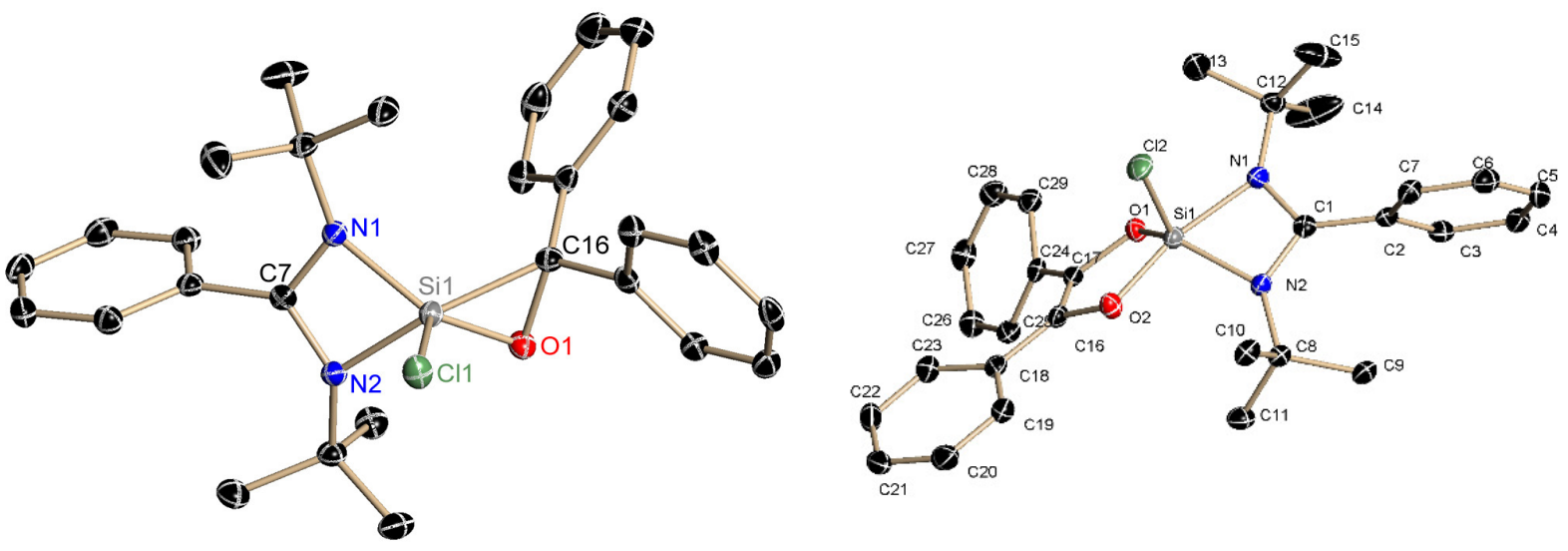


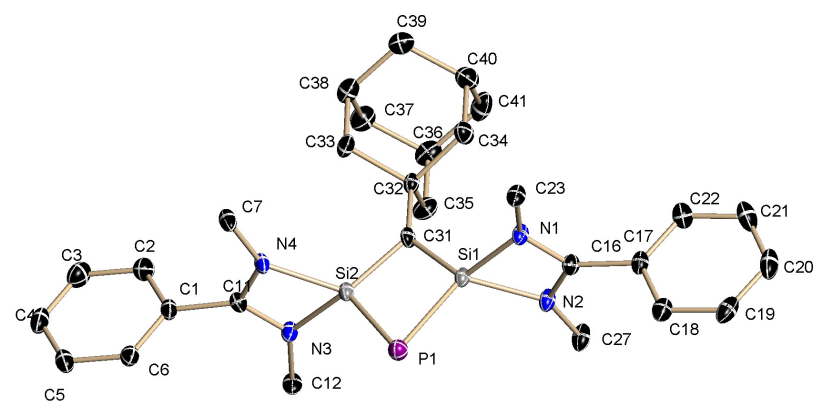

32

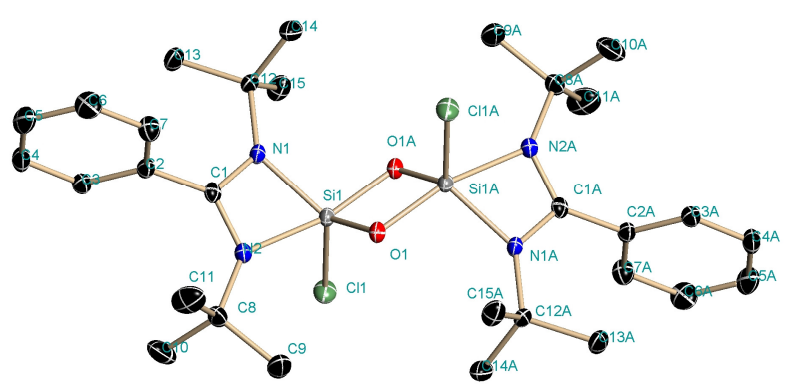

29

\subsection{Future Scope}

Extension of this work will surely yield many fascinating results. The reactivity study of $\mathrm{Si}-\mathrm{Si}$ and Ge-Ge bond is in its infancy. So far we were not able to insert another group 14 moiety into the Si-Si bond. Treatment of $\mathrm{PhC}(\mathrm{N} t \mathrm{Bu})_{2} \mathrm{SiCl}$ and $\mathrm{PhC}(\mathrm{N} t \mathrm{Bu})_{2} \mathrm{GeCl}$ with $\mathrm{LSi}-$ SiL may be an interesting approach. Further reduction may lead to the formation of silaallene, and mixed-allene derivative respectively.

Furthermore due to lack of time the reaction of LSi-SiL with transition metals was not performed which is very promising area, and may produce a variety of silylidyne complexes. These types of compounds are among the most important organometallic compounds, and great utility of transition metal alkylidyne complexes in organometallic and organic chemistry was highlighted by E.O. Fischer and R. R. Schrock in their noble lectures. ${ }^{138}$

Although we were able to isolate compounds containing Si-Si and Ge-Ge bonds, the alkine analogue of $\mathrm{Sn}-\mathrm{Sn}$ and $\mathrm{Pb}-\mathrm{Pb}$ bond stabilized by amidinatio ligand is still elusive. But recent experiments gave us an important clue that the substituent on the nitrogen atoms is playing pivotal role in stabilization of alkine analogue of group 14 elements. In the last chapter it was mentioned that the reduction of amidinato chlorostannylene with $t \mathrm{Bu}$ substituent on nitrogen 
leads to the formation of homoleptic tin(II) compound. Exchanging the $t \mathrm{Bu}$ group by 2,6diisoprpyl phenyl group would be the key to obtain the alkine analogues of tin and lead.

From the application point of view low valent group 14 elements are really potential precursors in material sciences. The capability of silylene to coordinate as a two-electron donor ligand to the transition metal complexes was already mentioned. Recently the Fischer group showed that $\left[\mathrm{GaCp}^{*}\right]$ and $\mathrm{Ga}$ [Dippnacnac] can be used as precursors for the synthesis of unforeseen molecular, metal rich Hume-Rothery type systems of inner metallic complexes. ${ }^{139}$ Similar type of reaction can be carried out with silylene and germylene. Very recently Fürstner et al. showed that stable silylene has the ability to be a ligand for cross coupling reaction like N-heterocyclic carbene. ${ }^{140}$ This research area is still unexplored, and I am sure in recent years many more striking and fascinating results will come.

The isolation of room temperature stable 1,4-disilabenzene is also of paramount importance. The chemical behavior of silabenzene can be compared with benzene and it would be of fundamental interest to carry out electrophilic aromatic substitution reaction with 1,4disilabenzene. Moreover, 1,4-digermabenzene is still not isolable, and $\mathbf{4 2}$ can be a good precursor for it. 
A work such as this is actually never complete.

One must declare it to be complete when one has done all that is possible given the time and the circumstances.

Johann Wolfgang von Goethe, “Italian Journey” (1787) 


\section{Chapter 9}

\section{Experimental Section}

\section{General Procedure}

All reactions and handling of reagents were performed under an atmosphere of dry nitrogen or argon using standard Schlenk techniques or a glove box where the $\mathrm{O}_{2}$ and $\mathrm{H}_{2} \mathrm{O}$ levels were usually kept below $1 \mathrm{ppm}$. All glassware was oven-dried at $150{ }^{\circ} \mathrm{C}$ for at least $24 \mathrm{~h}$, assembled hot and cooled under high vacuum prior to use. Toluene, $n$-hexane, $n$-pentane, tetrahydrofuran, and diethyl ether were purified with the M-Braun solvent drying system. Dichloromethane was dried over $\mathrm{CaH}_{2}$ and distilled prior to use.

\subsection{Physical Measurements}

Melting points were measured in sealed glass tubes on a Büchi B-540 melting point apparatus.

NMR spectra were recorded on Bruker Avance 200, Bruker Avance 300, and Bruker Avance 500 NMR spectrometers. Downfield shifts from the reference are quoted positive; upfield shifts are assigned negative values for the case of external references. Deuterated NMR solvents $\mathrm{C}_{6} \mathrm{D}_{6}, \mathrm{C}_{7} \mathrm{D}_{8}$, and THF- $\mathrm{D}_{8}$ were dried by stirring for 2 days over $\mathrm{Na} / \mathrm{K}$ alloy followed by distillation in vacuo and degassed. $\mathrm{CD}_{2} \mathrm{Cl}_{2}$ was stirred with $\mathrm{CaH}_{2}$ for $6 \mathrm{~h}$ followed by filtration. Heteroatom NMR spectra were recorded ${ }^{1} \mathrm{H}$ decoupled with the exception of ${ }^{19} \mathrm{~F}$. Chemical shifts are reported in ppm with reference to residual deuterated solvent peak (internal) for ${ }^{1} \mathrm{H}$ nuclei, $\mathrm{SnMe}_{4}$ (external) for ${ }^{119} \mathrm{Sn}$ nuclei, $\mathrm{CFCl}_{3}$ (external) for ${ }^{19} \mathrm{~F}$ nuclei, $85 \% \mathrm{H}_{3} \mathrm{PO}_{4}$ (external) for ${ }^{31} \mathrm{P}$ nuclei, $\mathrm{SiMe}_{4}$ (external) for ${ }^{29} \mathrm{Si}$ nuclei.

IR spectra were recorded on a Bio-Rad Digilab FTS7 spectrometer in the range 4000-350 $\mathrm{cm}^{-1}$ as $\mathrm{KBr}$ pellets. Only the absorption of significant moieties $(\mathrm{N}-\mathrm{H}, \mathrm{O}-\mathrm{H}, \mathrm{O}-\mathrm{D}, \mathrm{CO}, \mathrm{C}=\mathrm{C})$ are assigned. 
Mass spectra were obtained with a Finnigan MAT 8230 or a Varian MAT CH5 instrument (70 eV) by EI-MS methods.

Elemental analyses were performed by the Analytisches Labor des Instituts für Anorganische Chemie der Universität Göttingen.

Chemicals were purchased and used as received.

\subsection{Synthesis of compounds}

9.2.1 Preparation of 21: $\mathrm{PhLi}(6.86 \mathrm{~mL}, 13.72 \mathrm{mmol}, 1.8 \mathrm{M}$ in diethyl ether) was added to a solution of $t \mathrm{BuN}=\mathrm{C}=\mathrm{N} t \mathrm{Bu}(2.12 \mathrm{~g}, 13.72 \mathrm{mmol})$ in diethyl ether $(80 \mathrm{~mL})$ at $-78{ }^{\circ} \mathrm{C}$. The solution was raised to ambient temperature and stirred for $4 \mathrm{~h}$. To the solution $\mathrm{HSiCl}_{3}$ was added drop by drop $(4.25 \mathrm{~mL}, 17.17 \mathrm{mmol})$ at $-78{ }^{\circ} \mathrm{C}$. The reaction mixture was warmed to room temperature and was stirred for $12 \mathrm{~h}$. The precipitate was filtered off, and after removal of all volatiles the residue was extracted with toluene $(20 \mathrm{~mL})$.Storage of the extract at $-32{ }^{\circ} \mathrm{C}$ in a freezer for 1 day afforded colorless crystals of 21. Mp $145-150{ }^{\circ} \mathrm{C}$. Elemental analysis (\%) calcd for $\mathrm{C}_{15} \mathrm{H}_{24} \mathrm{Cl}_{2} \mathrm{~N}_{2} \mathrm{Si}$ (331.36): C, 54.37; H, 7.30; N, 8.45; found: C, 54.66; H, 7.63; N, 8.06. ${ }^{1} \mathrm{H}$ NMR $\left(500 \mathrm{MHz}, \mathrm{C}_{6} \mathrm{D}_{6}, 25^{\circ} \mathrm{C}\right): \delta 1.08$ (s, $\left.18 \mathrm{H}, t \mathrm{Bu}\right), 6.70(\mathrm{~s}, 1 \mathrm{H}, \mathrm{Si}-H), 7.41-7.48$ (m, 5H,Ph) ppm; ${ }^{13} \mathrm{C}\left\{{ }^{1} \mathrm{H}\right\} \mathrm{NMR}\left(125.75 \mathrm{MHz}, \mathrm{C}_{6} \mathrm{D}_{6}, 25^{\circ} \mathrm{C}\right): \delta 31.8\left(\mathrm{CMe}_{3}\right), 54.2\left(\mathrm{CMe}_{3}\right)$, 128.6, 128.9, 129.1, 129.9, 130.6, $135.6(\mathrm{Ph}), 173.3(\mathrm{NCN}) \mathrm{ppm} ;{ }^{29} \mathrm{Si}\left\{{ }^{1} \mathrm{H}\right\} \mathrm{NMR}(99.36 \mathrm{MHz}$, $\left.\mathrm{C}_{6} \mathrm{D}_{6}, 25^{\circ} \mathrm{C}\right): \delta-96.4$ ppm. EI-MS: $m / z(\%): 330\left[\mathrm{M}^{+}\right],(100)$.

9.2.2 New Synthesis of 18: Toluene $(50 \mathrm{~mL})$ was added to a mixture of $21(0.31 \mathrm{~g}, 1.00$ mmol) and bis-trimethylsilyl lithium amide $(0.17 \mathrm{~g}, 1.01 \mathrm{mmol})$ at ambient temperature. Immediately the solution turned to a red color with the formation of $\mathrm{LiCl}$. The resulting mixture was stirred overnight. The solvent was then removed in vacuo, and the residue was extracted with toluene $(20 \mathrm{~mL})$. The filtrate was concentrated to yield colorless crystals of $\mathbf{1 8}$ $(0.26 \mathrm{~g}, 90 \%)$ 
Alternative Method: Toluene (50 mL) was added to a mixture of $21(1.00 \mathrm{~g}, 3.03 \mathrm{mmol})$ and 1,3-di-tert-butylimidazol-2-ylidene $(0.6 \mathrm{~g}, 3.33 \mathrm{mmol})$ at ambient temperature. The resulting mixture was stirred overnight. The solvent was then removed in vacuo, and the residue was extracted with toluene $(50 \mathrm{~mL})$. The filtrate was concentrated to yield colorless crystals of $\mathbf{1 8}$ (0.32g, 35\%). Mp 159-162 ${ }^{\circ} \mathrm{C}$. Elemental analysis (\%) calcd for $\mathrm{C}_{15} \mathrm{H}_{23} \mathrm{ClN}_{2} \mathrm{Si}: \mathrm{C}, 61.10 ; \mathrm{H}$, 7.87; N, 9.51; found: C, 60.95; H, 7.64; N, 9.35. ${ }^{1} \mathrm{H}$ NMR (200 MHz, $\left.\mathrm{C}_{6} \mathrm{D}_{6}, 25{ }^{\circ} \mathrm{C}\right): \delta 1.08$ (s, $18 \mathrm{H}, t \mathrm{Bu}), 6.78-7.0(\mathrm{~m}, 5 \mathrm{H}, P h) \mathrm{ppm} ;{ }^{13} \mathrm{C}\left\{{ }^{1} \mathrm{H}\right\}$ NMR $\left(125.75 \mathrm{MHz}, \mathrm{C}_{6} \mathrm{D}_{6}, 25^{\circ} \mathrm{C}\right): \delta 31.4$ $\left(\mathrm{CMe}_{3}\right), 53.7\left(\mathrm{CMe}_{3}\right), 126.32,127.4,127.9,128.4,129.8,133.0(\mathrm{Ph}), 166.7(\mathrm{NCN}) \mathrm{ppm}$; ${ }^{29} \mathrm{Si}\left\{{ }^{1} \mathrm{H}\right\} \mathrm{NMR}\left(99.36 \mathrm{MHz}, \mathrm{C}_{6} \mathrm{D}_{6}, 25{ }^{\circ} \mathrm{C}\right): \delta 14.6 \mathrm{ppm}$. EI-MS: $m / z: 295\left[\mathrm{M}^{+}\right]$

9.2.3 Preparation of 22: To the mixture of $\mathbf{1 8}(0.30 \mathrm{~g}, 1.02 \mathrm{mmol})$ and biphenyl alkine $(0.10$ $\mathrm{g}, 0.60 \mathrm{mmol})$ toluene $(20 \mathrm{~mL})$ was added at room temperature. The mixture was stirred overnight. The solid was filtered off and the solution was concentrated and kept at room temperature for 4 days to yield colorless crystals of $22(0.11 \mathrm{~g}, 42 \%)$. Mp 165-170 ${ }^{\circ} \mathrm{C}$. Elemental analysis (\%) calcd for $\mathrm{C}_{44} \mathrm{H}_{56} \mathrm{Cl}_{2} \mathrm{~N}_{4} \mathrm{Si}_{2}: \mathrm{C}, 68.81 ; \mathrm{H}, 7.35 ; \mathrm{N}, 7.29$; found: C, 67.95; H, 7.04; N, 8.35. ${ }^{1} \mathrm{H}$ NMR (300 MHz, $\left.\mathrm{C}_{6} \mathrm{D}_{6}, 25{ }^{\circ} \mathrm{C}\right): \delta 1.15(\mathrm{~s}, 36 \mathrm{H}, t \mathrm{Bu}), 6.78-7.05$ $(\mathrm{m}, 10 \mathrm{H}, \mathrm{Ph}) ; 7.28-7.55(\mathrm{~m}, 10 \mathrm{H}, \mathrm{Ph}) \mathrm{ppm} ;{ }^{13} \mathrm{C}\left\{{ }^{1} \mathrm{H}\right\} \mathrm{NMR}\left(125.75 \mathrm{MHz}, \mathrm{C}_{6} \mathrm{D}_{6}, 25{ }^{\circ} \mathrm{C}\right): \delta$ $32.83\left(\mathrm{CMe}_{3}\right), 55.23\left(\mathrm{CMe}_{3}\right), 128.5,128.9,129.4,129.9,130.2,133.0(\mathrm{Ph}), 156.45(\mathrm{NCN})$ ppm; ${ }^{29} \mathrm{Si}\left\{{ }^{1} \mathrm{H}\right\}$ NMR $\left(99.36 \mathrm{MHz}, \mathrm{C}_{6} \mathrm{D}_{6}, 25{ }^{\circ} \mathrm{C}\right): \delta=-109.53 \mathrm{ppm}$. EI-MS: $m / z: 294\left[\mathrm{M}^{+}\right]$ $(100 \%)$.

9.2.4 Preparation of 23: To the mixture of $22(0.5 \mathrm{~g}, 0.65 \mathrm{mmol})$ and trimethyl tin fluoride $(0.24 \mathrm{~g}, 1.31 \mathrm{mmol})$ toluene $(25 \mathrm{~mL})$ was added and stirred until the solution becomes transparent. After filtration the solution was concentrated and kept at $-32{ }^{\circ} \mathrm{C}$ to get pure compound $23(0.2 \mathrm{~g}, 41 \%)$. Mp $182-185^{\circ} \mathrm{C} .{ }^{1} \mathrm{H}$ NMR $\left(300 \mathrm{MHz}, \mathrm{C}_{6} \mathrm{D}_{6}, 25^{\circ} \mathrm{C}\right): \delta 1.23(\mathrm{~s}$, $36 \mathrm{H}, t \mathrm{Bu}), 7.12-7.28(\mathrm{~m}, 10 \mathrm{H}, P h) ; 7.48-7.75(\mathrm{~m}, 10 \mathrm{H}, P h) \mathrm{ppm} ;{ }^{13} \mathrm{C}\left\{{ }^{1} \mathrm{H}\right\} \mathrm{NMR}(125.75$ $\left.\mathrm{MHz}, \mathrm{C}_{6} \mathrm{D}_{6}, 25{ }^{\circ} \mathrm{C}\right): \delta 32.4\left(\mathrm{CMe}_{3}\right), 53.7\left(\mathrm{CMe}_{3}\right), 127.4,127.9,128.4,129.8,133.0(P h)$, 
166.7 (NCN) ppm; ${ }^{19} \mathrm{~F}\left(188.31 \mathrm{MHz}, \mathrm{CFCl}_{3}, \mathrm{C}_{6} \mathrm{D}_{6}, 25^{\circ} \mathrm{C}\right): \delta-71.73\left(J_{\mathrm{Si}-\mathrm{F}}=254.22 \mathrm{~Hz}\right) \mathrm{ppm}$; ${ }^{29} \mathrm{Si}\left\{{ }^{1} \mathrm{H}\right\}$ NMR $\left(99.36 \mathrm{MHz}, \mathrm{C}_{6} \mathrm{D}_{6}, 25^{\circ} \mathrm{C}\right): \delta-117.82,-115.26$ (doublet $J_{\text {Si-F }}=254.22 \mathrm{~Hz}$ ) ppm. EI-MS: m/z: $283\left[\mathrm{M}^{+}\right](100 \%)$.

9.2.5 Preparation of 24: To a $100 \mathrm{~mL}$ Schlenk flask containing 18 (0.6 g, $2.04 \mathrm{mmol})$ and benzophenone $(0.37 \mathrm{~g}, 2.04 \mathrm{mmol})$ was added toluene $(30 \mathrm{~mL})$. The resulting colorless solution was stirred at room temperature over night. Removal of all volatiles under vacuum gave a white solid. Recrystallization from a saturated toluene solution at $0{ }^{\circ} \mathrm{C}$ yielded $0.68 \mathrm{~g}$, $70 \%$ of colorless crystals. Mp $158-163{ }^{\circ} \mathrm{C}$. Elemental analysis (\%) calcd for $\mathrm{C}_{28} \mathrm{H}_{33} \mathrm{ClN}_{2} \mathrm{OSi}$ : C 70.49, H 6.97, N 5.87; found: C 70.64, H 6.42, N 5.67. ${ }^{1} \mathrm{H}$ NMR $\left(500 \mathrm{MHz}, \mathrm{C}_{6} \mathrm{D}_{6}, 25{ }^{\circ} \mathrm{C}\right): \delta$ $1.02(\mathrm{~s} 18 \mathrm{H}, t \mathrm{Bu}), 6.86-7.43(\mathrm{~m}, 15 \mathrm{H}, \mathrm{Ph}) \mathrm{ppm} ;{ }^{13} \mathrm{C}\left\{{ }^{1} \mathrm{H}\right\}$ NMR $\left(125.77 \mathrm{MHz}, \mathrm{C}_{6} \mathrm{D}_{6}, 25{ }^{\circ} \mathrm{C}\right)$ : $\delta 30.80\left(\mathrm{CMe}_{3}\right), 54.54\left(\mathrm{CMe}_{3}\right), 83.16\left(\mathrm{SiCPh}_{2}\right), 125.05,127.80,127.91,128.00,128.06$, $128.12,128.19,128.29,128.58,130.17,130.34,131.12(\mathrm{Ph}), 176.43(\mathrm{NCN}) \mathrm{ppm} ;{ }^{29} \mathrm{Si} \mathrm{NMR}$ (59.62 MHz, $\left.\mathrm{C}_{6} \mathrm{D}_{6}, 25^{\circ} \mathrm{C}\right): \delta-115.53$ ppm. EI-MS: (m/z): $476.21(100 \%)$.

9.2.6 Preparation of 25 . Toluene $(25 \mathrm{~mL})$ was added to the mixture of $\mathbf{1 8}(0.29 \mathrm{~g}, 1.02$ mmol) and benzil $(0.21 \mathrm{~g}, 1.00 \mathrm{mmol})$ at ambient temperature. The mixture was stirred overnight. The solvent was removed under vacuum. The residue was treated with $20 \mathrm{~mL}$ toluene and filtered. Storage of the filtrate at $-32{ }^{\circ} \mathrm{C}$ in a freezer for 1 day afforded colorless crystals of $25(0.39 \mathrm{~g}, 71.4 \%)$. Mp $164-170{ }^{\circ} \mathrm{C}$. Elemental analysis (\%) calcd for $\mathrm{C}_{29} \mathrm{H}_{33} \mathrm{ClN}_{2} \mathrm{O}_{2} \mathrm{Si}$ (505.12): C, 68.96; H, 6.58; N, 5.55; found: C, 68.66; H, 6.43; N, 5.06. ${ }^{1} \mathrm{H}$ NMR $\left(500 \mathrm{MHz}, \mathrm{THF}^{\mathrm{D}} \mathrm{D}_{8}, 25{ }^{\circ} \mathrm{C}\right): \delta 1.20(\mathrm{~s}, 18 \mathrm{H}, t \mathrm{Bu}), 7.13-7.56(\mathrm{~m}, 15 \mathrm{H}, \mathrm{Ph}) \mathrm{ppm} ;$ ${ }^{13} \mathrm{C}\left\{{ }^{1} \mathrm{H}\right\}$ NMR $\left(125.75 \mathrm{MHz}, \mathrm{THF}-\mathrm{D}_{8}, 25^{\circ} \mathrm{C}\right): \delta 31.8\left(\mathrm{CMe}_{3}\right), 55.6\left(\mathrm{CMe}_{3}\right), 127.4,127.6$, $128.5,128.6,128.9,129.1,129.9,130.6,135.6,135.2,135.8(P h), 175.2(\mathrm{NCN}) \mathrm{ppm} ;$ ${ }^{29} \mathrm{Si}\left\{{ }^{1} \mathrm{H}\right\} \mathrm{NMR}\left(99.36 \mathrm{MHz}, \mathrm{C}_{6} \mathrm{D}_{6}, 25{ }^{\circ} \mathrm{C}\right): \delta-92.2 \mathrm{ppm}$. EI-MS: $m / z: 505\left[\mathrm{M}^{+}\right],(100 \%)$.

9.2.7 Preparation of 26. To the mixture of $\mathbf{1 8}(0.30 \mathrm{~g}, 1.02 \mathrm{mmol})$ and 2,6-diisopropylphenyl carbodiimide $(0.36 \mathrm{~g}, 0.99 \mathrm{mmol})$ toluene $(25 \mathrm{~mL})$ was added at room temperature. The 
mixture was stirred overnight. The solvent was removed under vacuum and the remaining solid was extracted with toluene $(20 \mathrm{~mL})$. The resulting solution was concentrated and stored at room temperature for 2 days to yield colorless crystals of 26 (0.11 g, 42\%). Mp 165-170 ${ }^{\circ} \mathrm{C}$. Elemental analysis (\%) calcd for $\mathrm{C}_{27} \mathrm{H}_{40} \mathrm{ClN}_{3} \mathrm{Si}$ (470.17): C, 68.97; H, 8.58; N, 8.94; found: $\mathrm{C}, 67.95 ; \mathrm{H}, 8.14 ; \mathrm{N}, 8.35 .{ }^{1} \mathrm{H}$ NMR $\left(500 \mathrm{MHz}, \mathrm{C}_{6} \mathrm{D}_{6}, 25{ }^{\circ} \mathrm{C}\right): \delta 1.20-1.21(\mathrm{~d}, 12 \mathrm{H}$, $\left.\mathrm{CH}_{3}\right), 1.25-1.27$ (d, $\left.18 \mathrm{H}, t \mathrm{Bu}\right) ; 3.5-3.6$ (sept, $1 \mathrm{H}, \mathrm{CHMe}$ ), $4.0-4.1$ (sept, $1 \mathrm{H}, \mathrm{CHMe}$ ), 7.04-7.23 (m, $8 \mathrm{H}, \mathrm{Ph}) \mathrm{ppm} ;{ }^{13} \mathrm{C}\left\{{ }^{1} \mathrm{H}\right\}$ NMR $\left(75.45 \mathrm{MHz}, \mathrm{C}_{6} \mathrm{D}_{6}, 25{ }^{\circ} \mathrm{C}\right): \delta 23.3(\mathrm{CHMe}), 28.4$ $\left(\mathrm{CHMe}_{2}\right), 32.9\left(\mathrm{CMe}_{3}\right), 54.9\left(\mathrm{CMe}_{3}\right), 117.3,122.5,123.2,133.4,138.4,140.4,142.9,146.6$, (Ph), 172.5 (NCN) ppm; ${ }^{29} \mathrm{Si}\left\{{ }^{1} \mathrm{H}\right\} \mathrm{NMR}\left(59.62 \mathrm{MHz}, \mathrm{C}_{6} \mathrm{D}_{6}, 25{ }^{\circ} \mathrm{C}\right): \delta-104.79 \mathrm{ppm} . \mathrm{EI}-\mathrm{MS}$ : $m / z: 469\left[\mathrm{M}^{+}\right](100 \%)$.

9.2.8 Preparation of 27: To the mixture of $\mathbf{1 8}(0.3 \mathrm{~g}, 1.02 \mathrm{mmol})$ and adamantyl azide $(0.18$ $\mathrm{g}, 1.02 \mathrm{mmol})$ toluene $(25 \mathrm{~mL})$ was added at room temperature and stirred overnight. After the removal of the solvent in vacuum, the solid was extracted with toluene $(30 \mathrm{~mL})$. Upon concentration and storage of the solution at room temperature afforded colorless solid 27 (0.19 g, 40\%). Mp $212-220^{\circ} \mathrm{C}$. Elemental analysis (\%) calcd for $\mathrm{C}_{30} \mathrm{H}_{46} \mathrm{Cl}_{2} \mathrm{~N}_{4} \mathrm{O}_{2} \mathrm{Si}_{2}(620.25)$ : C, 57.95; H, 7.46; N, 9.01 found: C, 57.02; H, 7.14; N, 8.95; ${ }^{1} \mathrm{H}$ NMR $\left(500 \mathrm{MHz}, \mathrm{C}_{6} \mathrm{D}_{6}\right.$, $\left.25^{\circ} \mathrm{C}\right): \delta 1.42(\mathrm{~s}, 36 \mathrm{H}, t \mathrm{Bu}), 6.86-7.13(\mathrm{~m}, 10 \mathrm{H}, P h) \mathrm{ppm} ;{ }^{13} \mathrm{C}\left\{{ }^{1} \mathrm{H}\right\} \mathrm{NMR}(75.45 \mathrm{MHz}$, $\left.\mathrm{C}_{6} \mathrm{D}_{6}, 25^{\circ} \mathrm{C}\right): \delta 32.27\left(\mathrm{CMe}_{3}\right), 54.2\left(\mathrm{CMe}_{3}\right), 127.5,128.0,128.5,128.9,129.2,137.8(P h)$ $172.69(\mathrm{NCN}) \mathrm{ppm} ;{ }^{29} \mathrm{Si}\left\{{ }^{1} \mathrm{H}\right\} \mathrm{NMR}\left(59.62 \mathrm{MHz}, \mathrm{C}_{6} \mathrm{D}_{6}, 25{ }^{\circ} \mathrm{C}\right): \delta-103.54 \mathrm{ppm} . \mathrm{EI}-\mathrm{MS}: \mathrm{m} / z$ : $620\left[\mathrm{M}^{+}\right]$

9.2.9 Preparation of 28: To the mixture of $\mathbf{1 8}(0.294 \mathrm{~g}, 1.00 \mathrm{mmol})$ and 1,3,5,7-octatetraene $(0.104 \mathrm{~g}, 1.00 \mathrm{mmol})$ toluene $(30 \mathrm{~mL})$ was added and the resulting solution was stirred overnight. The solvent was then removed in vacuo, and the residue was extracted with toluene $(20 \mathrm{~mL})$. The filtrate was concentrated to yield colorless crystals of $28(0.29 \mathrm{~g}, 75 \%)$. Mp $175-179{ }^{\circ} \mathrm{C}$. Elemental analysis $(\%)$ calcd for $\mathrm{C}_{23} \mathrm{H}_{31} \mathrm{ClN}_{2} \mathrm{Si}$ (398.19): C, 69.23; H, 7.83; N, 
7.02; found: $\mathrm{C}, 68.96 ; \mathrm{H}, 7.02 ; \mathrm{N}, 7.55 .{ }^{1} \mathrm{H}$ NMR $\left(200 \mathrm{MHz}, \mathrm{C}_{6} \mathrm{D}_{6}, 25{ }^{\circ} \mathrm{C}\right): \delta 1.12$ (br, $18 \mathrm{H}$, $t \mathrm{Bu}), 2.28(\mathrm{br}, 2 \mathrm{H}, \mathrm{CH}), 5.63-5.93(\mathrm{~m}, 6 \mathrm{H}, \mathrm{COT}), 6.76-7.00(\mathrm{~m}, 5 \mathrm{H}, \mathrm{Ph}) \mathrm{ppm} ;{ }^{13} \mathrm{C}\left\{{ }^{1} \mathrm{H}\right\} \mathrm{NMR}$ (125.75 MHz, $\left.\mathrm{C}_{6} \mathrm{D}_{6}, 25^{\circ} \mathrm{C}\right): \delta 31.8\left(\mathrm{CMe}_{3}\right), 37.7(\mathrm{CH}), 54.7\left(\mathrm{CMe}_{3}\right), 125.8,127.5,128.0$, 128.5, 128.9, 129.3, $135.9(P h), 169.3(\mathrm{NCN}) \mathrm{ppm} ;{ }^{29} \mathrm{Si}\left\{{ }^{1} \mathrm{H}\right\}$ NMR (99.36 MHz, $\mathrm{C}_{6} \mathrm{D}_{6}$, $\left.25^{\circ} \mathrm{C}\right): \delta$-9.49 ppm. EI-MS: $m / z: 397\left[\mathrm{M}^{+}\right](40 \%)$.

9.2.10 Preparation of 29: To the mixture of $\mathbf{1 8}(0.29 \mathrm{~g}, 1.00 \mathrm{mmol})$ and tert-butylisocyanate $(0.10 \mathrm{~g}, 1.01 \mathrm{mmol})$ toluene $(25 \mathrm{~mL})$ was added and the solution was stirred overnight. After the removal of the solvent in vacuum, the solid was extracted with toluene $(30 \mathrm{~mL})$. Upon concentration and storage of the solution at room temperature afforded colorless crystals of 29 $(0.24 \mathrm{~g}, 40 \%)$. Mp $182-185^{\circ} \mathrm{C}$. Elemental analysis (\%) calcd for $\mathrm{C}_{30} \mathrm{H}_{46} \mathrm{Cl}_{2} \mathrm{~N}_{4} \mathrm{O}_{2} \mathrm{Si}_{2}$ (620.25): C, 57.95; H, 7.46; N, 9.01 found: C, 57.02; H, 7.14; N, 8.95. ${ }^{1} \mathrm{H}$ NMR (500 MHz, $\left.\mathrm{C}_{6} \mathrm{D}_{6}, 25^{\circ} \mathrm{C}\right): \delta 1.42(\mathrm{~s}, 36 \mathrm{H}, t \mathrm{Bu}), 6.86-7.13(\mathrm{~m}, 10 \mathrm{H}, \mathrm{Ph}) \mathrm{ppm} ;{ }^{13} \mathrm{C}\left\{{ }^{1} \mathrm{H}\right\}$ NMR $(75.45$ $\left.\mathrm{MHz}, \mathrm{C}_{6} \mathrm{D}_{6}, 25^{\circ} \mathrm{C}\right): \delta 32.27\left(\mathrm{CMe}_{3}\right), 54.2\left(\mathrm{CMe}_{3}\right), 127.5,128.0,128.5,128.9,129.2,137.8$ (Ph) $172.69(\mathrm{NCN}) \mathrm{ppm} ;{ }^{29} \mathrm{Si}\left\{{ }^{1} \mathrm{H}\right\}$ NMR $\left(59.62 \mathrm{MHz}, \mathrm{C}_{6} \mathrm{D}_{6}, 25^{\circ} \mathrm{C}\right): \delta-113.54 \mathrm{ppm} . \mathrm{EI}-\mathrm{MS}$ : $m / z: 620\left[\mathrm{M}^{+}\right]$.

Alternative preparation of 29: To the mixture of $\mathbf{1 8}(0.3 \mathrm{~g}, 1.02 \mathrm{mmol})$ and trimethyl-Noxide (0.08 g, $1.06 \mathrm{mmol})$ THF (25 mL) was added and stirred until the solution becomes transparent. After the removal of the solvent in vacuum, the solid was extracted with toluene (30 mL).Upon concentration and storage of the solution at $-32{ }^{\circ} \mathrm{C}$ afforded colorless crystals of 29 ( $0.24 \mathrm{~g}, 40 \%)$. The formation of $\mathbf{2 9}$ is confirmed by comparing the spectroscopic data with the previous compound.

9.2.11 Preparation of 30: Toluene $(40 \mathrm{~mL})$ was added to a mixture of $\mathbf{1 8}(0.29 \mathrm{~g}, 1.00 \mathrm{mmol})$ and glyoxal-bis-(2,6-diisopropylphenyl)imine $(0.38 \mathrm{~g}, 1.01 \mathrm{mmol})$ at ambient temperature. The resulting mixture was stirred overnight. The solvent was then removed in vacuo, and the residue was extracted with toluene $(20 \mathrm{~mL})$. The filtrate was concentrated to yield colorless 
crystals of 30 (0.35 g, 52.2\%). Mp 189-192 ${ }^{\circ} \mathrm{C}$. Elemental analysis (\%) calcd for $\mathrm{C}_{41} \mathrm{H}_{59} \mathrm{ClN}_{4} \mathrm{Si}$ (671.47): C, 73.34; H, 8.86; N, 8.34; found: C, 73.67; H, 9.02; N, 9.35; ${ }^{1} \mathrm{H}$ NMR (200 MHz, $\left.\mathrm{C}_{6} \mathrm{D}_{6}, 25^{\circ} \mathrm{C}\right): \delta 1.20(\mathrm{~s}, 24 \mathrm{H}, \mathrm{CHMe} 2), 1.30$ (s, $\left.18 \mathrm{H}, t \mathrm{Bu}\right), 2.91$ (sept, 4H, $\left.\mathrm{C} H \mathrm{Me}_{2}\right), 6.2(\mathrm{~s}, 2 \mathrm{H}, \mathrm{CHCN}), 6.74-7.12(\mathrm{~m}, \mathrm{Ph}) \mathrm{ppm} ;{ }^{13} \mathrm{C}\left\{{ }^{1} \mathrm{H}\right\} \mathrm{NMR}\left(125.75 \mathrm{MHz}, \mathrm{C}_{6} \mathrm{D}_{6}\right.$, $\left.25^{\circ} \mathrm{C}\right): \delta 23.4\left(\mathrm{CHMe}_{2}\right), 28.9\left(\mathrm{CHMe}_{2}\right), 32.1\left(\mathrm{CMe}_{3}\right), 54.3\left(\mathrm{CMe}_{3}\right), 119.8(\mathrm{NCC}), 122.9$, $123.6,124.3,125.5,126.1,127.8,128.1,129.2,130.0,133.8,144.8,145.7,146.9,148.4$, 149.1, $149.6(\mathrm{Ph}), 169.4(\mathrm{NCN}) \mathrm{ppm} ;{ }^{29} \mathrm{Si}\left\{{ }^{1} \mathrm{H}\right\} \mathrm{NMR}\left(59.62 \mathrm{MHz}, \mathrm{C}_{6} \mathrm{D}_{6}, 25^{\circ} \mathrm{C}\right): \delta-101.89$ ppm. EI-MS: m/z: $670\left[\mathrm{M}^{+}\right](100 \%)$.

9.2.12 Preparation of 31: To the mixture of $\mathbf{1 8}(0.29 \mathrm{~g}, 1.00 \mathrm{mmol})$ and biphenylbisulfide $(0.22 \mathrm{~g}, 1.00 \mathrm{mmol})$ toluene $(25 \mathrm{~mL})$ was added and stirred till the solution became transparent. The solvent was removed under reduced pressure and the crude product was extracted with toluene $(2 \mathrm{~mL})$. Upon concentration and storing at $-32{ }^{\circ} \mathrm{C}$ in a freezer afforded yellowish white solid. Elemental analysis (\%) calcd for $\mathrm{C}_{27} \mathrm{H}_{33} \mathrm{ClN}_{2} \mathrm{~S}_{2} \mathrm{Si}$ (512.15) C, 63.19; H, 6.48; N, 5.46; found: C, 62.96; H, 6.02; N, 6.12; ${ }^{1} \mathrm{H}$ NMR $\left(200 \mathrm{MHz}, \mathrm{C}_{6} \mathrm{D}_{6}, 25{ }^{\circ} \mathrm{C}\right): \delta 1.42(\mathrm{~s}$, $18 \mathrm{H}, t \mathrm{Bu}), 6.90-7.10(\mathrm{~m}, 11 \mathrm{H}, \mathrm{Ph}), 7.88-7.91(\mathrm{~m}, 4 \mathrm{H}, \mathrm{Ph}) \mathrm{ppm} ;{ }^{13} \mathrm{C}\left\{{ }^{1} \mathrm{H}\right\} \mathrm{NMR}(125.75$ $\left.\mathrm{MHz}, \mathrm{C}_{6} \mathrm{D}_{6}, 25^{\circ} \mathrm{C}\right): \delta 32.2\left(\mathrm{CMe}_{3}\right), 56.2\left(\mathrm{CMe}_{3}\right), 127.5,127.81,128.0,128.3,129.1,129.8$ 133.8, 135.8, $136.6(P h), 170.7(\mathrm{NCN}) \mathrm{ppm} ;{ }^{29} \mathrm{Si}\left\{{ }^{1} \mathrm{H}\right\} \mathrm{NMR}\left(99.36 \mathrm{MHz}, \mathrm{C}_{6} \mathrm{D}_{6}, 25{ }^{\circ} \mathrm{C}\right): \delta-$ 83.26 ppm. EI-MS: m/z: 477 [M-Cl] (100\%).

9.2.13 Preparation of 32: To the mixture of $\mathbf{1 8}(0.29 \mathrm{~g}, 1.00 \mathrm{mmol})$ and adamantyl phosphaalkine $(0.07 \mathrm{~g}, 0.38 \mathrm{mmol})$ at $-20{ }^{\circ} \mathrm{C}$ toluene $(25 \mathrm{~mL})$ was added and the color of the solution immediately changed from colorless to red. The reaction mixture was stirred overnight. The solvent was removed under reduced pressure and the crude product was extracted with toluene $(10 \mathrm{~mL})$. Upon concentration and storing at $0{ }^{\circ} \mathrm{C}$ in a freezer afforded reddish yellow crystals of 32. ${ }^{1} \mathrm{H}$ NMR $\left(200 \mathrm{MHz}, \mathrm{C}_{6} \mathrm{D}_{6}, 25{ }^{\circ} \mathrm{C}\right): \delta 1.26(\mathrm{~s}, 36 \mathrm{H}, t \mathrm{Bu}), 1.21$ (s, 4H, Ad), 1.72 (s, 12H, Ad), 6.90-7.10 (m, $10 \mathrm{H}, \mathrm{Ph}) \mathrm{ppm} ;{ }^{31} \mathrm{P}$ NMR (121.5 MHz, $\mathrm{C}_{6} \mathrm{D}_{6}, 25$ 
$\left.{ }^{\circ} \mathrm{C}\right)-243 \mathrm{ppm} ;{ }^{29} \mathrm{Si}\left\{{ }^{1} \mathrm{H}\right\} \mathrm{NMR}\left(99.36 \mathrm{MHz}, \mathrm{C}_{6} \mathrm{D}_{6}, 25^{\circ} \mathrm{C}\right): \delta-5.1 \mathrm{ppm}$; EI-MS: $m / z: 696$ (100\%).

9.2.14 Preparation of 33: The prepared $0.64 \mathrm{M}$ solution of $\mathrm{Ni}(\mathrm{CO})_{4}$ in ethyl ether $(1.75 \mathrm{~mL}$, $1.12 \mathrm{mmol})$ was added to the solution of $\mathbf{1 8}(0.31 \mathrm{~g}, 1.05 \mathrm{mmol})$ in toluene $(50 \mathrm{~mL})$ at ambient temperature. The color of the solution changed slowly from yellow to colorless. The mixture was stirred overnight. The reaction mixture was then filtered through celite and the solution was concentrated and stored at $4{ }^{\circ} \mathrm{C}$ overnight to yield colorless crystals of $\mathbf{3 3}(0.25$ g, 54.5\%). Mp $168-175^{\circ} \mathrm{C}$. Elemental analysis (\%) calcd for $\mathrm{C}_{18} \mathrm{H}_{23} \mathrm{ClN}_{2} \mathrm{NiO}_{3} \mathrm{Si}$ (438): C, 49.40; H, 5.30; N, 6.40. Found: C, 50.71; H, 5.95; N, 6.68. ${ }^{1} \mathrm{H}$ NMR $\left(200 \mathrm{MHz}, \mathrm{C}_{6} \mathrm{D}_{6}, 25{ }^{\circ} \mathrm{C}\right)$ : $\delta 1.06(\mathrm{~s}, 18 \mathrm{H}, t \mathrm{Bu}), 6.71-6.95(\mathrm{~m}, 5 \mathrm{H}, \mathrm{Ph}) \mathrm{ppm} ;{ }^{13} \mathrm{C}\left\{{ }^{1} \mathrm{H}\right\} \mathrm{NMR}\left(125.75 \mathrm{MHz}, \mathrm{C}_{6} \mathrm{D}_{6}, 25{ }^{\circ} \mathrm{C}\right)$ : $\delta 30.87\left(\mathrm{CMe}_{3}\right), 54.8\left(\mathrm{CMe}_{3}\right), 125.64,127.81,128.0,128.65,129.28,130.94(P h), 171.13$ (NCN), 199.31 (CO) ppm; ${ }^{29} \mathrm{Si}\left\{{ }^{1} \mathrm{H}\right\}$ NMR $\left(99.36 \mathrm{MHz}, \mathrm{C}_{6} \mathrm{D}_{6}, 25{ }^{\circ} \mathrm{C}\right): \delta 62.69 \mathrm{ppm} . \mathrm{EI}-\mathrm{MS}$ : $m / z(100 \%)$ 438. IR (Nujol, $\left.\mathrm{cm}^{-1}\right) \tilde{v}: 2060,1984$.

9.2.15 Preparation of 34: THF $(20 \mathrm{~mL})$ was added to the mixture of $\mathbf{1 8}(0.3 \mathrm{~g}, 1.02 \mathrm{mmol})$ and diiron nonacarbonyl $(0.26 \mathrm{~g}, 1.02 \mathrm{mmol})$ at ambient temperature under $\mathrm{N}_{2}$. After stirring for $2 \mathrm{~d}$ the initially yellow solution became darker in color to ultimately afford a garnet brown solution. Solvent was then removed in vacuum, and the residue was extracted with toluene (30 mL). The insoluble solid was filtered off. The garnet brown filtrate was concentrated and stored at $-30{ }^{\circ} \mathrm{C}$ to yield red brown solid of $34(0.24 \mathrm{~g}, 61 \%)$. Mp $168-175^{\circ} \mathrm{C}$. Elemental analysis (\%) calcd for $\mathrm{C}_{19} \mathrm{H}_{23} \mathrm{ClFeN}_{2} \mathrm{O}_{4} \mathrm{Si}$ (462.78): C, 49.31; H, 5.01; N, 6.05. Found: C, 51.92; H, 5.95; N, 6.14. ${ }^{1} \mathrm{H}$ NMR (200 MHz, $\left.\mathrm{C}_{6} \mathrm{D}_{6}, 25^{\circ} \mathrm{C}\right): \delta 1.01(\mathrm{~s}, 18 \mathrm{H}, t \mathrm{Bu}), 6.52-6.89$ (m, 5H, Ph) ppm; ${ }^{13} \mathrm{C}\left\{{ }^{1} \mathrm{H}\right\} \mathrm{NMR}\left(125.75 \mathrm{MHz}, \mathrm{C}_{6} \mathrm{D}_{6}, 25{ }^{\circ} \mathrm{C}\right): \delta 28.17\left(\mathrm{CMe}_{3}\right), 50.8\left(\mathrm{CMe}_{3}\right)$, 123.64, 124.56, 125.45, 126.78, 129.32, $130.44(P h), 168.87(\mathrm{NCN}), 196.47(C \mathrm{O}) \mathrm{ppm}$; ${ }^{29} \mathrm{Si}\left\{{ }^{1} \mathrm{H}\right\}$ NMR (99.36 MHz, $\left.\mathrm{C}_{6} \mathrm{D}_{6}, 25{ }^{\circ} \mathrm{C}\right): \delta 43.19 \mathrm{ppm}$. EI-MS: $\mathrm{m} / z, 462$ (100\%). IR (Nujol, $\left.\mathrm{cm}^{-1}\right) \tilde{v}: 2096,2025$. 
9.2.16 Preparation of 35: THF (50 mL) was added to a mixture of 20a (1.49 g, $4.08 \mathrm{mmol})$ and potassium graphite $(1.65 \mathrm{~g}, 12.24 \mathrm{mmol})$ at $-78^{\circ} \mathrm{C}$. The resulting red mixture was stirred overnight. The solvent was then removed in vacuo, and the residue was extracted with toluene $(50 \mathrm{~mL})$. The insoluble precipitate was filtered off and the red filtrate was concentrated to yield orange-red crystals of $35(0.11 \mathrm{~g}, 5.21 \%)$. Mp: $155-160{ }^{\circ} \mathrm{C} .{ }^{1} \mathrm{H}$ NMR $(200 \mathrm{MHz}$, THF$\left.d_{8}, 25^{\circ} \mathrm{C}\right): \delta 1.23(\mathrm{~s}, 36 \mathrm{H}, t \mathrm{Bu}), 7.34-7.38(\mathrm{~m}, 10 \mathrm{H}, \mathrm{Ph}) \mathrm{ppm} ;{ }^{13} \mathrm{C}\left\{{ }^{1} \mathrm{H}\right\} \mathrm{NMR}(125.75 \mathrm{MHz}$, THF- $\left.d_{8}, 25^{\circ} \mathrm{C}\right): \delta 32.19\left(\mathrm{CMe}_{3}\right), 53.36\left(\mathrm{CMe}_{3}\right), 128.42,129.91,130.95,131.95,134.35$, 136.49, $(P h), 146.32(\mathrm{NCN}) \mathrm{ppm} ;{ }^{29} \mathrm{Si}\left\{{ }^{1} \mathrm{H}\right\} \mathrm{NMR}\left(59.25 \mathrm{MHz}, \mathrm{THF}-d_{8}, 25^{\circ} \mathrm{C}\right): \delta 75.71 \mathrm{ppm} ;$ ${ }^{1} \mathrm{H}$ NMR $\left(200 \mathrm{MHz}, \mathrm{C}_{6} \mathrm{D}_{6}, 25^{\circ} \mathrm{C}\right): \delta 1.18(\mathrm{~s}, 36 \mathrm{H}, t \mathrm{Bu}), 7.22-7.30(\mathrm{~m}, 10 \mathrm{H}, P h) \mathrm{ppm}$; ${ }^{13} \mathrm{C}\left\{{ }^{1} \mathrm{H}\right\} \mathrm{NMR}\left(500 \mathrm{MHz}, \mathrm{C}_{6} \mathrm{D}_{6}, 25^{\circ} \mathrm{C}\right): \delta 32.01\left(\mathrm{CMe}_{3}\right), 53.73\left(\mathrm{CMe}_{3}\right), 127.71,128.19$, 128.40, 129.21, 130.32, 135.98, $(P h), 145.67(\mathrm{NCN}) \mathrm{ppm} ;{ }^{29} \mathrm{Si}\left\{{ }^{1} \mathrm{H}\right\} \mathrm{NMR}\left(500 \mathrm{MHz}, \mathrm{C}_{6} \mathrm{D}_{6}\right.$, $\left.25^{\circ} \mathrm{C}\right): \delta 76.29 \mathrm{ppm}$. EI-MS: $m / z(\%): 518.3\left[\mathrm{M}^{+}\right](100)$. Elemental analysis $(\%)$ calcd for $\mathrm{C}_{30} \mathrm{H}_{46} \mathrm{~N}_{4} \mathrm{Si}_{2}$ (518.33): C 69.44; H 8.94; N 10.80; found: C,68.26; H, 8.63; N,11.06.

9.2.17 Preparation of 36: THF (20 mL) was added to the mixture of $\mathbf{3 5}(0.5 \mathrm{gm}, 0.96 \mathrm{mmol})$ and benzophenone $(0.35 \mathrm{gm}, 1.92 \mathrm{mmol})$ at ambient temperature. The mixture was stirred overnight. The solution was removed in vacuo and $n$-hexane $(20 \mathrm{~mL})$ was added to it. The reaction mixture once again stirred overnight. Hexane was removed in vacuum and toluene (5 $\mathrm{mL}$ ) was added to the reaction mixture and the solution was concentrated and stored at room temperature for two days to yield colorless crystals of $36(0.22 \mathrm{~g}, 26 \%)$. Mp $165-170{ }^{\circ} \mathrm{C}$ Elemental analysis (\%) calcd for $\mathrm{C}_{70} \mathrm{H}_{84} \mathrm{~N}_{4} \mathrm{O}_{2} \mathrm{Si}_{2}$ (885.34) $\mathrm{C}, 75.97 ; \mathrm{H}, 7.74 ; \mathrm{N}, 6.33$; found: $\mathrm{C}, 77.56 ; \mathrm{H}, 8.04 ; \mathrm{N}, 5.35 ;{ }^{1} \mathrm{H} \mathrm{NMR}\left(300 \mathrm{MHz}, \mathrm{C}_{6} \mathrm{D}_{6}, 25 \mathrm{C}\right): \delta 1.27(\mathrm{~s}, 18 \mathrm{H}, t \mathrm{Bu}), 1.30$ (s, $18 \mathrm{H}, t \mathrm{Bu}), 4.1(\mathrm{~s}, 2 \mathrm{H}, \mathrm{CH}), 7.26-7.33(\mathrm{~m}, 10 \mathrm{H}, P h), 7.52-7.83(\mathrm{~m}, 10 \mathrm{H}, P h) \mathrm{ppm} ;{ }^{13} \mathrm{C}\left\{{ }^{1} \mathrm{H}\right\}$ $\operatorname{NMR}\left(125.75 \mathrm{MHz}, \mathrm{C}_{6} \mathrm{D}_{6}, 25^{\circ} \mathrm{C}\right): \delta 30.1\left(\mathrm{CMe}_{3}\right), 32.83\left(\mathrm{CMe}_{3}\right), 37.5(C \mathrm{H}), 44.9\left(\mathrm{CMe}_{3}\right)$, $57.25\left(\mathrm{CMe}_{3}\right), 128.5,128.9,129.4,129.9,130.2,133.0(\mathrm{Ph}), 156.45(\mathrm{NCN}) \mathrm{ppm} ;{ }^{29} \mathrm{Si}\left\{{ }^{1} \mathrm{H}\right\}$ NMR (99.36 MHz, $\left.\mathrm{C}_{6} \mathrm{D}_{6}, 25{ }^{\circ} \mathrm{C}\right): \delta-86.248 \mathrm{ppm}$. EI-MS: m/z: $884\left[\mathrm{M}^{+}\right](100 \%)$. 
9.2.18 Preparation of 37 . Toluene $(20 \mathrm{~mL})$ was added to a mixture of $\mathbf{3 5}(0.1 \mathrm{~g}, 0.19 \mathrm{mmol})$ and benzil $(0.08 \mathrm{~g}, 0.37 \mathrm{mmol})$ at ambient temperature. The mixture was stirred overnight. The volatiles were removed in vacuo and $n$-hexane $(10 \mathrm{~mL})$ was added to the residue. The reaction mixture once again was stirred overnight. The $n$-hexane was removed in vacuo and toluene $(10 \mathrm{~mL})$ was added to the reaction mixture. The solution was concentrated and stored at room temperature for two days to yield colorless crystals of $37 \cdot 0.5$ toluene $(0.07 \mathrm{~g}, 38.9 \%)$. Mp 154-158 ${ }^{\circ} \mathrm{C}$. For the elemental analysis 37 was treated in vacuo overnight. Anal. calcd for $37 \cdot 0.5$ toluene, $\mathrm{C}_{61.5} \mathrm{H}_{70} \mathrm{~N}_{4} \mathrm{O}_{4} \mathrm{Si}_{2}(985.39) \mathrm{C}, 74.96 ; \mathrm{H}, 7.16 ; \mathrm{N}, 5.69$; found: $\mathrm{C}, 74.51 ; \mathrm{H}$, 7.22; N, 5.55. ${ }^{1} \mathrm{H}$ NMR (500 MHz, $\left.\mathrm{C}_{6} \mathrm{D}_{6}, 25^{\circ} \mathrm{C}\right): \delta 1.42(\mathrm{~s}, 36 \mathrm{H}, t \mathrm{Bu}), 6.29(\mathrm{~s}, 2 \mathrm{H}, \mathrm{CH})$, 6.86-6.96 (m, $10 \mathrm{H}, P h) ; 7.92-8.19(\mathrm{~m}, 10 \mathrm{H}, P h) \mathrm{ppm} ;{ }^{13} \mathrm{C}\left\{{ }^{1} \mathrm{H}\right\}$ NMR (125.75 MHz, $\mathrm{C}_{6} \mathrm{D}_{6}$, $\left.25^{\circ} \mathrm{C}\right): \delta 30.8\left(\mathrm{CMe}_{3}\right), 57.2\left(\mathrm{CMe}_{3}\right), 125.6,126.4,127.0,127.8,127.9,128.5,128.9,129.4$, 129.9, 130.2, $133.0(P h), 171.37(\mathrm{NCN}) \mathrm{ppm} ;{ }^{29} \mathrm{Si}\left\{{ }^{1} \mathrm{H}\right\} \mathrm{NMR}\left(99.36 \mathrm{MHz}, \mathrm{C}_{6} \mathrm{D}_{6}, 25{ }^{\circ} \mathrm{C}\right): \delta-$ 79.75 ppm. EI-MS: m/z: $939\left[\mathrm{M}^{+}\right](100 \%)$.

9.2.19 Preparation of 38. Dry $\mathrm{N}_{2} \mathrm{O}$ was bubbled into a solution of $\mathbf{3 5}(0.11 \mathrm{~g}, 0.21 \mathrm{mmol})$ in toluene $(20 \mathrm{~mL})$ at room temperature. After $5 \mathrm{~min}$ the gas flow of $\mathrm{N}_{2} \mathrm{O}$ was disconnected, and all the volatiles were removed in vacuum. The residue was treated with $n$-hexane $(10 \mathrm{~mL})$ and stirred overnight. The $n$-hexane was removed in vacuum and toluene $(15 \mathrm{~mL})$ was added to the reaction mixture and the solution was concentrated and stored at room temperature to yield colorless crystals of $\mathbf{3 8} \cdot$ toluene $(0.12 \mathrm{~g}, 50 \%)$. Mp $168-175^{\circ} \mathrm{C}$. For the elemental analysis $\mathbf{3 8}$ toluene was kept in vacuum overnight to remove the toluene $(\%)$ calcd for $\mathrm{C}_{60} \mathrm{H}_{92} \mathrm{~N}_{8} \mathrm{O}_{6} \mathrm{Si}_{4}(1132.62) \mathrm{C}, 63.56 ; \mathrm{H}, 8.18 ; \mathrm{N}, 9.88$; found: $\mathrm{C}, 64.71 ; \mathrm{H}, 8.55 ; \mathrm{N}, 9.09 .{ }^{1} \mathrm{H}$ NMR $\left(500 \mathrm{MHz}, \mathrm{C}_{6} \mathrm{D}_{6}, 25{ }^{\circ} \mathrm{C}\right): \delta 1.58(\mathrm{~s}, 36 \mathrm{H}, t \mathrm{Bu}), 6.94-7.14(\mathrm{~m}, 20 \mathrm{H}, P h) \mathrm{ppm} ;{ }^{13} \mathrm{C}\left\{{ }^{1} \mathrm{H}\right\}$ NMR (125.75 MHz, $\left.\mathrm{C}_{6} \mathrm{D}_{6}, 25^{\circ} \mathrm{C}\right): \delta 33.1\left(\mathrm{CMe}_{3}\right), 53.5\left(\mathrm{CMe}_{3}\right), 125.6,127.6,128.3,128.5$, 128.8, 129.3, $135.9(P h), 171.23(\mathrm{NCN}) \mathrm{ppm} ;{ }^{29} \mathrm{Si}\left\{{ }^{1} \mathrm{H}\right\} \mathrm{NMR}\left(99.36 \mathrm{MHz}, \mathrm{C}_{6} \mathrm{D}_{6}, 25{ }^{\circ} \mathrm{C}\right): \delta-$ 111.02 ppm. EI-MS: m/z: $1132\left[\mathrm{M}^{+}\right](100 \%)$. 
Alternative Synthesis of 38. $t \mathrm{BuN}=\mathrm{C}=\mathrm{O}(0.99 \mathrm{~g}, 1.00 \mathrm{mmol})$ and $35(0.52 \mathrm{~g}, 1.00 \mathrm{mmol})$ were weighed in the same $100 \mathrm{~mL}$ Schlenk flask and toluene $(30 \mathrm{~mL})$ was charged into the reaction mixture at room temperature. The color of the reaction immediately turned from deep red to colorless. The reaction mixture was stirred for $5 \mathrm{~min}$, and concentrated to yield colorless crystals of 38. The multinuclear NMR spectroscopy corroborated the formation of 38.

9.2.20 Preparation of 39. THF $(25 \mathrm{~mL})$ was charged to the mixture of white phosphorus $(0.03 \mathrm{~g}, 0.21 \mathrm{mmol})$ and $35(0.11 \mathrm{~g}, 0.21 \mathrm{mmol})$ at room temperature. The color of the solution changed immediately from deep red to faint red. The reaction mixture was stirred for $2 \mathrm{~h}$ and all the volatiles were removed in vacuum. The residue was treated with THF $(10 \mathrm{~mL})$ and filtered through celite. The solution was concentrated and stored at $-32{ }^{\circ} \mathrm{C}$ to yield yellow crystals of $39(0.12 \mathrm{~g}, 50 \%)$. Mp $168-175^{\circ} \mathrm{C} .{ }^{1} \mathrm{H}$ NMR $\left(500 \mathrm{MHz}, \mathrm{C}_{6} \mathrm{D}_{6}, 25^{\circ} \mathrm{C}\right): \delta$ $1.36(\mathrm{~s}, 36 \mathrm{H}, t \mathrm{Bu}), 6.61-6.98(\mathrm{~m}, 10 \mathrm{H}, P h) \mathrm{ppm} ;{ }^{13} \mathrm{C}\left\{{ }^{1} \mathrm{H}\right\}$ NMR $\left(125.75 \mathrm{MHz}, \mathrm{C}_{6} \mathrm{D}_{6}\right.$, $\left.25^{\circ} \mathrm{C}\right): \delta 33.1\left(\mathrm{CMe}_{3}\right), 53.5\left(\mathrm{CMe}_{3}\right), 125.6,127.6,128.3,128.5,128.8,129.3,135.9(P h)$, $171.23(\mathrm{NCN})$ ppm; ${ }^{31} \mathrm{P}$ NMR $\left(121.5 \mathrm{MHz}, \mathrm{C}_{6} \mathrm{D}_{6}, 25{ }^{\circ} \mathrm{C}\right): \delta-166.02 \mathrm{ppm} ;{ }^{29} \mathrm{Si}\left\{{ }^{1} \mathrm{H}\right\} \mathrm{NMR}$ (99.36 MHz, $\left.\mathrm{C}_{6} \mathrm{D}_{6}, 25^{\circ} \mathrm{C}\right): \delta 25.6 \mathrm{ppm}$. EI-MS: $m / z: 580\left[\mathrm{M}^{+}\right](100 \%)$.

9.2.21 Preparation of 40. Toluene $(20 \mathrm{~mL})$ was added to a mixture of $\mathbf{3 5}(0.1 \mathrm{~g}, 0.19 \mathrm{mmol})$ and biphenyl alkine $(0.07 \mathrm{~g}, 0.39 \mathrm{mmol})$ at ambient temperature. The mixture was stirred overnight. The volatiles were removed in vacuo and cyclopentane $(10 \mathrm{~mL})$ was added to the residue. The reaction mixture once again was stirred overnight. The cyclopentane was removed in vacuum and THF $(2 \mathrm{~mL})$ and toluene $(10 \mathrm{~mL})$ were added to the reaction mixture. The solution was concentrated and stored at room temperature for two days to yield dark red crystals of $40(0.05 \mathrm{~g}, 29.41 \%)$. Mp $154-158{ }^{\circ} \mathrm{C}$. Anal. calcd for $\mathbf{4 0}, \mathrm{C}_{58} \mathrm{H}_{66} \mathrm{~N}_{4} \mathrm{Si}_{2}$ (874.34) C, 79.58; H, 7.60; N, 6.40 found: C, 80.51; H, 7.29; N, 6.55; Due to the high sensitivity of the crystals the reported values deviate from the calculated one. ${ }^{1} \mathrm{H}$ NMR $\left(200 \mathrm{MHz}, \mathrm{C}_{6} \mathrm{D}_{6}\right.$, 
$\left.25^{\circ} \mathrm{C}\right): \delta 1.31(\mathrm{~s}, 36 \mathrm{H}, t \mathrm{Bu}), 6.86-6.96(\mathrm{~m}, 10 \mathrm{H}, \mathrm{Ph}) ; 7.92-8.19 \mathrm{ppm}(\mathrm{m}, 20 \mathrm{H}, \mathrm{Ph}){ }^{13} \mathrm{C}\left\{{ }^{1} \mathrm{H}\right\}$ NMR (125.75 MHz, $\left.\mathrm{C}_{6} \mathrm{D}_{6}, 25{ }^{\circ} \mathrm{C}\right): \delta 30.8\left(\mathrm{CMe}_{3}\right), 57.2\left(\mathrm{CMe}_{3}\right), 125.6,126.4,127.0,127.8$, $127.9,128.5,128.9,129.4,129.9,130.2,133.0,136.4,138.9,139.0,140.2,141.5(P h), 171.37$ $(\mathrm{NCN}) \mathrm{ppm} ;{ }^{29} \mathrm{Si}\left\{{ }^{1} \mathrm{H}\right\} \mathrm{NMR}\left(99.36 \mathrm{MHz}, \mathrm{C}_{6} \mathrm{D}_{6}, 25{ }^{\circ} \mathrm{C}\right): \delta-18.05 \mathrm{ppm}$. EI-MS: $m / z: 874\left[\mathrm{M}^{+}\right]$ $(30 \%)$.

9.2.22 Preparation of Compound 41: $\mathrm{PhLi}(6.86 \mathrm{~mL}, 13.72 \mathrm{mmol}, 1.8 \mathrm{M}$ in diethyl ether) was added to a solution of $t \mathrm{BuN}=\mathrm{C}=\mathrm{N} t \mathrm{Bu}(2.12 \mathrm{~g}, 13.72 \mathrm{mmol})$ in diethyl ether $(80 \mathrm{~mL})$ at $78^{\circ} \mathrm{C}$. The solution was raised to ambient temperature and stirred for $4 \mathrm{~h}$. The solution was added drop by drop to a stirred suspension of $\mathrm{GeCl}_{2} \cdot$ dioxane $(3.18 \mathrm{~g}, 13.72 \mathrm{mmol})$ in diethyl ether $(20 \mathrm{~mL})$ at $-78{ }^{\circ} \mathrm{C}$. The reaction mixture was warmed to room temperature and was stirred for $24 \mathrm{~h}$. The precipitate was filtered off, and after removal of all volatiles, the residue was extracted with toluene $(20 \mathrm{~mL})$. Storage of the extract at $-32{ }^{\circ} \mathrm{C}$ in a freezer for 1 day afforded colorless crystals of 41. Mp 145-150 ${ }^{\circ} \mathrm{C}$. Elemental analysis (\%) calcd for $\mathrm{C}_{15} \mathrm{H}_{23} \mathrm{ClGeN}_{2}$ (338.45): C, 53.18; H, 6.77; N, 8.27; found: $\mathrm{C}, 52.66 ; \mathrm{H}, 6.63 ; \mathrm{N}, 8.06 .{ }^{1} \mathrm{H}$ NMR (200 MHz, THF- $\left.d_{8}, 25^{\circ} \mathrm{C}\right): \delta 1.08 \mathrm{ppm}(\mathrm{s}, 18 \mathrm{H}, t \mathrm{Bu}), 7.41-7.48 \mathrm{ppm}(\mathrm{m}, 5 \mathrm{H}, \mathrm{Ph})$; ${ }^{13} \mathrm{C}\left\{{ }^{1} \mathrm{H}\right\}$ NMR $\left(125.75 \mathrm{MHz}, \mathrm{THF}-d_{8}, 25^{\circ} \mathrm{C}\right): \delta 31.8\left(\mathrm{CMe}_{3}\right), 54.2\left(\mathrm{CMe}_{3}\right), 128.6,128.9$,

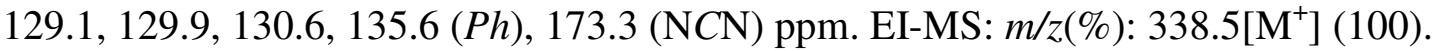

9.2.23 Preparation of Compound 42: THF (50 mL) was added to a mixture of $41(1.42 \mathrm{~g}$, $4.18 \mathrm{mmol})$ and finely divided potassium $(0.25 \mathrm{~g}, 6.25 \mathrm{mmol})$ at ambient temperature. The resulting red mixture was stirred for 2 days. The solvent was then removed in vacuo, and the residue was extracted with toluene $(50 \mathrm{~mL})$. The insoluble precipitate was filtered off and the red filtrate was concentrated to yield crystals of $\mathbf{4 2}(0.89 \mathrm{~g}, 35 \%)$. Mp 220-225 ${ }^{\circ} \mathrm{C}$. Elemental analysis (\%) calcd for $\mathrm{C}_{30} \mathrm{H}_{46} \mathrm{Ge}_{2} \mathrm{~N}_{4}$ (607.99): C, 59.26; H, 7.63; N, 9.22; found: $\mathrm{C}, 57.52 ; \mathrm{H}$, $7.47 ; \mathrm{N}, 8.54$. Due to the sensitivity of the crystals the analytical values show some deviation from the calcd one. ${ }^{1} \mathrm{H}$ NMR $\left(200 \mathrm{MHz}, \mathrm{THF}-d_{8}, 25^{\circ} \mathrm{C}\right): \delta 1.16(\mathrm{~s}, 36 \mathrm{H}, t \mathrm{Bu}), 7.34-7.39(\mathrm{~m}$, 
$10 \mathrm{H}, \mathrm{Ph}) \mathrm{ppm} ;{ }^{13} \mathrm{C}\left\{{ }^{1} \mathrm{H}\right\} \mathrm{NMR}\left(125.75 \mathrm{MHz}, \mathrm{THF}-d_{8}, 25^{\circ} \mathrm{C}\right): \delta 31.9\left(\mathrm{CMe}_{3}\right), 32.1\left(\mathrm{CMe}_{3}\right)$, $53.5\left(\mathrm{CMe}_{3}\right), 55.0\left(\mathrm{CMe}_{3}\right), 123.0,126.9,127.8,128.1,128.3,128.9,129.1,129.4,129.9$, 130.5, 131.5, $138.0(\mathrm{Ph}), 154.5(\mathrm{NCN}), 164.3(\mathrm{NCN})$ ppm. EI-MS: $m / z(\%): 608.5\left[\mathrm{M}^{+}\right](100)$. 9.2.24 Preparation of 44: A mixture of $42(0.2 \mathrm{~g}, 0.33 \mathrm{mmol})$ and $\mathrm{PhN}=\mathrm{NPh}(0.06 \mathrm{~g}$, $0.33 \mathrm{mmol})$ in toluene $(20 \mathrm{~mL})$ was stirred at room temperature overnight. The solvent was removed under reduced pressure, and the remaining powder was washed with $n$-hexane (10 $\mathrm{mL})$. Further recrystallisation from toluene $(5 \mathrm{~mL})$ at room temperature afforded colorless crystals of $44(0.16 \mathrm{~g}, 61.5 \%)$. Mp $148-155^{\circ} \mathrm{C}$ Elemental analysis (\%) calcd for $\mathrm{C}_{42} \mathrm{H}_{56} \mathrm{Ge}_{2} \mathrm{~N}_{6}$ (885.34) C, 75.97; H, 7.74; N, 6.33; found: C, 77.56; H, 8.04; N, 5.35. ${ }^{1} \mathrm{H}$ NMR (300 MHz, $\left.\mathrm{C}_{6} \mathrm{D}_{6}, 25^{\circ} \mathrm{C}\right): \delta 1.27(\mathrm{~s}, 18 \mathrm{H}, t \mathrm{Bu}), 1.30(\mathrm{~s}, 18 \mathrm{H}, t \mathrm{Bu}), 4.1(\mathrm{~s}, 2 \mathrm{H}, C \mathrm{H}), 7.26-7.33(\mathrm{~m}, 10 \mathrm{H}$, $\mathrm{Ph}) ;$ 7.52-7.83 (m, $10 \mathrm{H}, \mathrm{Ph}) \mathrm{ppm} ;{ }^{13} \mathrm{C}\left\{{ }^{1} \mathrm{H}\right\} \mathrm{NMR}\left(125.75 \mathrm{MHz}, \mathrm{C}_{6} \mathrm{D}_{6}, 25{ }^{\circ} \mathrm{C}\right): \delta 30.1\left(\mathrm{CMe} e_{3}\right)$, $32.83\left(\mathrm{CMe}_{3}\right), 37.5(\mathrm{CH}), 44.9\left(\mathrm{CMe}_{3}\right), 57.25\left(\mathrm{CMe}_{3}\right), 128.5,128.9,129.4,129.9,130.2$, $133.0(\mathrm{Ph}), 156.45(\mathrm{NCN}) \mathrm{ppm} . \mathrm{EI}-\mathrm{MS}: \mathrm{m} / z: 790\left[\mathrm{M}^{+}\right](100 \%)$.

9.2.25 Preparation of 45: THF was added to the mixture of $\mathbf{4 2}(0.2 \mathrm{~g}, 0,33 \mathrm{mmol})$ and diiron nonacarbonyl $(0.24 \mathrm{~g}, 0.67 \mathrm{mmol})$ at ambient temperature under $\mathrm{N}_{2}$. After stirring for $40 \mathrm{~h}$ the initially light orange solution became darker in color to ultimately afford a garnet brown solution. Solvent was then removed in vacuum, and the residue was extracted with toluene (30 mL). The insoluble solid was filtered off. The garnet brown filtrate was concentrated and stored at $-30{ }^{\circ} \mathrm{C}$ to yield red brown solid of 45 (0.52 g, 61\%). Mp 180-185 ${ }^{\circ} \mathrm{C}$. Elemental analysis (\%) Calcd for $\mathrm{C}_{38} \mathrm{H}_{46} \mathrm{Fe}_{2} \mathrm{Ge}_{2} \mathrm{~N}_{4} \mathrm{O}_{8}$ (943.67): C, 48.36; $\mathrm{H}, 4.91 ; \mathrm{N}$, 5.94; found: $\mathrm{C}$, 47.51; $\mathrm{H}, 4.33 ; \mathrm{N}, 5.85 .{ }^{1} \mathrm{H}$ NMR $\left(200 \mathrm{MHz}, \mathrm{C}_{6} \mathrm{D}_{6}, 25^{\circ} \mathrm{C}\right): \delta 1.41(\mathrm{~s}, 36 \mathrm{H}, t \mathrm{Bu}), 7.56-7.82(\mathrm{~m}$, 5H, Ph) ppm; ${ }^{13} \mathrm{C}\left\{{ }^{1} \mathrm{H}\right\} \mathrm{NMR}\left(125.75 \mathrm{MHz}, \mathrm{C}_{6} \mathrm{D}_{6}, 25^{\circ} \mathrm{C}\right): \delta 31.3\left(\mathrm{CMe}_{3}\right), 54.9\left(\mathrm{CMe}_{3}\right), 127.8$, 128.2, 128.3, 129.2, 130.0, $130.7(P h), 171.1(\mathrm{NCN}), 220.5(C \mathrm{O}) \mathrm{ppm} . \mathrm{IR}\left(\mathrm{Nujol}, \mathrm{cm}^{-1}\right): \tilde{v}$ (2029(m), 1974(s), 1920(s) (CO). EI-MS: m/z(\%): 943[M+], (100). 
9.2.25 Preparation of 46. $\mathrm{PhLi}(6.86 \mathrm{~mL}, 13.72 \mathrm{mmol}, 1.8 \mathrm{~m}$ in diethyl ether) was added to a solution of $t \mathrm{BuN}=\mathrm{C}=\mathrm{N} t \mathrm{Bu}(2.12 \mathrm{~g}, 13.72 \mathrm{mmol})$ in diethyl ether $(80 \mathrm{~mL})$ in a $250 \mathrm{~mL}$ Schlenk flask at $-78{ }^{\circ} \mathrm{C}$. The solution was raised to ambient temperature and stirred for $4 \mathrm{~h}$. The solution was added drop by drop to a stirred suspension of $\mathrm{SnCl}_{2}(3.18 \mathrm{~g}, 13.72 \mathrm{mmol})$ in diethyl ether $(20 \mathrm{~mL})$ at $-78{ }^{\circ} \mathrm{C}$. The reaction mixture was warmed to room temperature and was stirred for $24 \mathrm{~h}$. The precipitate was filtered off, and after removal of all volatiles in vacuum, the residue was extracted with toluene $(20 \mathrm{~mL})$. Storage of the extract at $-32{ }^{\circ} \mathrm{C}$ in a freezer for 1 day afforded colorless crystals of 46. Mp $135-140{ }^{\circ} \mathrm{C}$. Elemental analysis (\%) Calcd for $\mathrm{C}_{15} \mathrm{H}_{23} \mathrm{ClN}_{2} \mathrm{Sn}$ (385.52): C, 46.73; H, 6.01; N, 7.27; found: C, 46.96; H, 6.33; N, 7.56. ${ }^{1} \mathrm{H}$ NMR $\left(200 \mathrm{MHz}, \mathrm{THF}-d_{8}, 25^{\circ} \mathrm{C}\right): \delta 1.08(\mathrm{~s}, 18 \mathrm{H}, t \mathrm{Bu}), 7.41-7.48(\mathrm{~m}, 5 \mathrm{H}, \mathrm{Ph}) \mathrm{ppm}$; ${ }^{13} \mathrm{C}\left\{{ }^{1} \mathrm{H}\right\} \mathrm{NMR}\left(500 \mathrm{MHz}, \mathrm{THF}-d_{8}, 25^{\circ} \mathrm{C}\right): \delta 31.8\left(\mathrm{CMe}_{3}\right), 54.2\left(\mathrm{CMe}_{3}\right), 128.6,128.9,129.1$, 129.9, 130.6, $135.6(P h), 173.3(\mathrm{NCN}) \mathrm{ppm} ;{ }^{119} \mathrm{Sn} \mathrm{NMR}\left(500 \mathrm{~Hz}, \mathrm{THF}-d_{8}, 25^{\circ} \mathrm{C}\right): \delta 29.6$ ppm. EI-MS: $m / z(\%): 385.5\left[\mathrm{M}^{+}\right],(100)$

9.2.26 Preparation of 47. A solution of $\mathbf{4 6}(0.385 \mathrm{~g}, 1.0 \mathrm{mmol})$ in toluene $(20 \mathrm{~mL})$ was added to a stirred suspension of $\mathrm{AgSO}_{3} \mathrm{CF}_{3}(0.257 \mathrm{~g}, 1.0 \mathrm{mmol})$ in toluene $(10 \mathrm{~mL})$ at room temperature and was stirred for $4 \mathrm{~h}$. The precipitate was filtered off, and the solvent was partially removed (ca. $15 \mathrm{~mL}$ ) under reduced pressure. Storage of the remaining solution in a $10{ }^{\circ} \mathrm{C}$ freezer for 2 days afforded colorless crystals of 47 suitable for X-ray diffraction analyses (0.55 g, 80\%). Mp 135-140 ${ }^{\circ} \mathrm{C}$. Elemental analysis(\%) calcd. for $\mathrm{C}_{16} \mathrm{H}_{23} \mathrm{~F}_{3} \mathrm{~N}_{2} \mathrm{O}_{3} \mathrm{SSn}$ (500.04): C, 38.50; H, 4.64; N, 5.61; found: C, 38.96; H, 4.75; N, 6.01. ${ }^{1} \mathrm{H}$ NMR (200 MHz, THF- $\left.d_{8}, 25^{\circ} \mathrm{C}\right): \delta 1.08(\mathrm{~s}, 18 \mathrm{H}, t \mathrm{Bu}), 7.41-7.48(\mathrm{~m}, 5 \mathrm{H}, \mathrm{Ph}) \mathrm{ppm} ;{ }^{13} \mathrm{C}\left\{{ }^{1} \mathrm{H}\right\} \mathrm{NMR}(125.75 \mathrm{MHz}$, THF- $\left.d_{8}, 25^{\circ} \mathrm{C}\right): \delta 32.7\left(\mathrm{CMe}_{3}\right), 53.0\left(\mathrm{CMe}_{3}\right), 127.3,127.5,128.0,128.4,128.7,129.3(\mathrm{Ph})$, $168.0(\mathrm{NCN}) \mathrm{ppm} ;{ }^{119} \mathrm{Sn}$ NMR $\left(111.92 \mathrm{~Hz}, \mathrm{THF}-d_{8}, 25^{\circ} \mathrm{C}\right): \delta-33.16 \mathrm{ppm} ;{ }^{19} \mathrm{~F}$ NMR $(188.3$ Hz, THF- $\left.d_{8}, 25^{\circ} \mathrm{C}\right): \delta-73.6$ ppm. EI-MS: $m / z(\%): 500\left[\mathrm{M}^{+}\right],(100)$. 
9.2.27 Preparation of 48. In a $100 \mathrm{~mL}$ Schlenk flask compound 46 and $\mathrm{LiN}\left(\mathrm{SiMe}_{3}\right)_{2}$ were placed and diethylether $(40 \mathrm{~mL})$ was added to the flask at room temperature. The reaction mixture was stirred overnight. A precipitate was formed and filtered off. The solvent was partially removed in vaccuo. Storage of the remaining solution at $-30{ }^{\circ} \mathrm{C}$ overnight in a freezer resulted in colorless crystals of $\mathbf{4 8}$ suitable for $\mathrm{X}$ ray analysis. Mp 120-125 ${ }^{\circ} \mathrm{C}$. Elemental analysis (\%) calcd for $\mathrm{C}_{21} \mathrm{H}_{41} \mathrm{~N}_{3} \mathrm{Si}_{2} \mathrm{Sn}$ (511.19): C, 49.41; H, 8.10; N, 8.23; found: C, 48.96; H, 8.33; N, 7.56. ${ }^{1} \mathrm{H}$ NMR $\left(200 \mathrm{MHz}, \mathrm{THF}-d_{8}, 25^{\circ} \mathrm{C}\right): \delta 0.25(\mathrm{~s}, 18 \mathrm{H}, T M S), 1.27(\mathrm{~s}, 18 \mathrm{H}$, $t \mathrm{Bu}), 7.33-7.43(\mathrm{~m}, 5 \mathrm{H}, \mathrm{Ph}) \mathrm{ppm} ;{ }^{13} \mathrm{C}\left\{{ }^{1} \mathrm{H}\right\} \mathrm{NMR}\left(125.75 \mathrm{MHz}, \mathrm{THF}-d_{8}, 25^{\circ} \mathrm{C}\right): \delta 25.3$ $\left(\mathrm{SiMe}_{3}\right), 32.9\left(\mathrm{CMe}_{3}\right), 53.7\left(\mathrm{CMe}_{3}\right), 128.2,128.3,128.6,129.1,129.7,130.1(\mathrm{Ph}), 169.3$ (NCN) ppm; ${ }^{119} \mathrm{Sn}$ NMR $\left(111.92 \mathrm{~Hz}, \mathrm{THF}-d_{8}, 25^{\circ} \mathrm{C}\right): \delta-33.58 \mathrm{ppm} ;{ }^{29} \mathrm{Si} \mathrm{NMR}(59.63 \mathrm{~Hz}$, THF- $\left.d_{8}, 25^{\circ} \mathrm{C}\right): \delta 1.49$ ppm. EI-MS: $m / z(\%): 511\left[\mathrm{M}^{+}\right],(100)$.

9.2.28 Preparation of 49. : A solution of $\mathrm{K}\left[\mathrm{HB}(\mathrm{sec}-\mathrm{Bu})_{3}\right]$ in $\mathrm{THF}(2.00 \mathrm{~mL}, 1 \mathrm{~m}$ in THF) was slowly added drop by drop to a stirred solution of $\mathbf{4 6}(1.050 \mathrm{~g}, 2 \mathrm{mmol})$ in toluene $(30 \mathrm{~mL})$ at $-10{ }^{\circ} \mathrm{C}$. The reaction mixture was warmed to room temperature and then stirred for additional $1 \mathrm{~h}$. After removal of all the volatiles, the residue was extracted with toluene $(30 \mathrm{~mL})$, concentrated to $10 \mathrm{~mL}$, and stored in a $-30{ }^{\circ} \mathrm{C}$ freezer. Colorless crystals of $\mathbf{4 9}$ were formed after 1 day. Mp 135-140 ${ }^{\circ} \mathrm{C}$. Elemental analysis (\%) calcd for $\mathrm{C}_{30} \mathrm{H}_{46} \mathrm{~N}_{2} \mathrm{Sn}$ (581.42): C, 65.11; H, 8.38; N, 5.06; found: C, 64.96; H, 8.33; N, 5.56. ${ }^{1} \mathrm{H}$ NMR $\left(200 \mathrm{MHz}, \mathrm{THF}-d_{8}, 25^{\circ} \mathrm{C}\right): \delta$ $1.08(\mathrm{~s}, 36 \mathrm{H}, t \mathrm{Bu}), 7.41-7.48(\mathrm{~m}, 10 \mathrm{H}, \mathrm{Ph}) \mathrm{ppm} ;{ }^{13} \mathrm{C}\left\{{ }^{1} \mathrm{H}\right\} \mathrm{NMR}\left(125.75 \mathrm{MHz}, \mathrm{THF}-d_{8}, 25^{\circ} \mathrm{C}\right)$ : $\delta 31.8\left(\mathrm{CMe}_{3}\right), 54.2\left(\mathrm{CMe}_{3}\right), 128.6,128.9,129.1,129.9,130.6,135.6(\mathrm{Ph}), 173.3(\mathrm{NCN})$ ppm; ${ }^{119}$ Sn NMR $\left(111.92 \mathrm{~Hz}, \mathrm{THF}-d_{8}, 25^{\circ} \mathrm{C}\right): \delta-285$ ppm. EI-MS: $m / z(\%): 581\left[\mathrm{M}^{+}\right],(30)$.

9.2.29 Preparation of 50. THF (30 mL) was added to the mixture of $\mathbf{4 6}(0.4 \mathrm{~g}, 1.03 \mathrm{mmol})$ and diiron nonacarbonyl $(0.45 \mathrm{~g}, 1.23 \mathrm{mmol})$ at ambient temperature under $\mathrm{N}_{2}$. After stirring for $40 \mathrm{~h}$ the initially light orange solution became darker in color to ultimately afford a garnet brown solution. The solvent was then removed in vacuum, and the residue was extracted with 
toluene $(30 \mathrm{~mL})$. The insoluble solid was filtered off. The garnet brown filtrate was concentrated and stored at $-30{ }^{\circ} \mathrm{C}$ to yield red brown solid of $\mathbf{5 0}(0.52 \mathrm{~g}, 61 \%)$. Mp: $182-189$ ${ }^{\circ} \mathrm{C}$. Elemental analysis (\%) calcd for $\mathrm{C}_{19} \mathrm{H}_{23} \mathrm{ClFeN}_{2} \mathrm{O}_{4} \mathrm{Sn}$ (553.40): C, 41.24; H, 4.19; N, 5.06; found: $\mathrm{C}, 43.51 ; \mathrm{H}, 5.33 ; \mathrm{N}, 4.85$. Due to sensitivity of the crystals towards air and moisture the reported values deviate from the calcd. one. ${ }^{1} \mathrm{H}$ NMR $\left(200 \mathrm{MHz}, \mathrm{THF}-d_{8}, 25^{\circ} \mathrm{C}\right): \delta 1.41$ $(\mathrm{s}, 18 \mathrm{H}, t \mathrm{Bu}), 7.56-7.82(\mathrm{~m}, 5 \mathrm{H}, \mathrm{Ph}) \mathrm{ppm} ;{ }^{13} \mathrm{C}\left\{{ }^{1} \mathrm{H}\right\} \mathrm{NMR}\left(125.75 \mathrm{MHz}, \mathrm{THF}-d_{8}, 25^{\circ} \mathrm{C}\right): \delta$ $31.3\left(\mathrm{CMe}_{3}\right), 54.9\left(\mathrm{CMe}_{3}\right), 127.8,128.2,128.3,129.2,130.0,130.7(\mathrm{Ph}), 171.1(\mathrm{NCN}), 220.5$ (CO) ppm; ${ }^{119} \mathrm{Sn}$ NMR $\left(111.92 \mathrm{~Hz}, \mathrm{THF}-d_{8}, 25^{\circ} \mathrm{C}\right): \delta 255 \mathrm{ppm}$; EI-MS: $m / z(\%): 553\left[\mathrm{M}^{+}\right]$, (100).

\subsection{Crystal Structure Determination}

Shock cooled crystals were selected and mounted under nitrogen atmosphere using the XTEMP2. ${ }^{44}$ The structure was solved by direct methods (SHELXS) and refined on $F^{2}$ using full matrix least squares methods of SHELXL. ${ }^{44 \mathrm{~b}}$

The data of $22, \mathbf{2 4}, \mathbf{2 8}, \mathbf{2 9}, \mathbf{3 0}, \mathbf{3 2}, \mathbf{3 7}, \mathbf{3 8}, \mathbf{3 9}$, and 40 were measured on a INCOATEC Mo Microsource with Quazar mirror optics. ${ }^{44 \mathrm{~d}}$ 25, 26, 36, 44 and 45 were measured on a Bruker TXS-Mo rotating anode. Both had a APEX II detector on a D8 goniometer. The diffractometers where equipped with a low temperature device and used $\mathrm{MoK}_{\alpha}$ radiation, $\lambda=$ $0.71 \AA$. The data sets were integrated with SAINT $^{141}$ and an empirical absorption (SADABS) was applied. ${ }^{142}$

The data of 33, 41, and $\mathbf{4 2}$ were collected on a Bruker three-circle diffractometer equipped with a SMART $6000 \mathrm{CCD}$ detector and a mirror-system-monochromated $\mathrm{Cu} \mathrm{K}_{\alpha}$ source. The data of structures $\mathbf{4 6}, \mathbf{4 7}, \mathbf{4 8}$, and 49 were collected on a Bruker three-circle diffractometer equipped with a SMART $6000 \mathrm{CCD}$ detector and a mirror-system-monochromated $\mathrm{CuK}_{\alpha}$ source. The data of $\mathbf{3 3}$ was collected on an IPDS II Stoe image-plate diffractometer (graphite 
monochromated Mo $\mathrm{K}_{\alpha}$ radiation, $\lambda=0.71073 \AA$ ) at $133(2) \mathrm{K}$. The data were integrated with SAINT and an empirical absorption correction with SADABS was applied. ${ }^{143,144}$

The structures were solved by direct methods (SHELXS-97) and refined by full-matrix leastsquares methods against $F^{2}$ (SHELXL-97). ${ }^{4 \mathrm{~b}}$ All non-hydrogen-atoms were refined with anisotropic displacement parameters. The hydrogen atoms were refined isotropically on calculated positions using a riding model with their $U_{\text {iso }}$ values constrained to equal to 1.5 times the $U_{\text {eq }}$ of their pivot atoms for terminal $\mathrm{sp}^{3}$ carbon atoms and 1.2 times for all other carbon atoms. Disordered moieties were refined using bond lengths restraints and isotropic displacement parameters restraints.

In compound $\mathbf{4 6}$ one $t \mathrm{Bu}$ group was modeled in two conformations (occupancy of the minor component was refined to $0.40(1)$ ). The disordered group was refined with distance restraints and restraints for the anisopropic displacement parameters.

The structure of $\mathbf{4 8}$ was refined as a pseudo-merohedral twin (twin fraction 0.473(2)) in space group $P-1$ with two molecules in the asymmetric unit. Also a refinement in space group $P 2{ }_{1} / c$ with a disordered model was possible, but showed the following features: systematic absences violations, high residual density peak of $0.75 \mathrm{e} / \AA^{3}$, higher $R 1$ and $w R 2$ values, higher standard uncertainties for bond length and angles, strange proposed weighting scheme, high $\mathrm{K}$ (mean $F_{o}{ }^{2} / F_{c}{ }^{2}$ ) for the reflections with the lowest intensities.

Compound 49 crystallizes as a non-merohedral twin (twin fraction 0.0252(9)) in the monoclinic space group $C 2 / c$. The twin law is a twofold rotation about the real axis 001 .

All the structures are published. The data can be obtained free of charge from either $\underline{\text { www.ccdc.cam.ac.uk/data_request/cif }}$ or www.pubs.acs.org. 


\subsection{Handling and Disposal of Solvents and Residual Waste}

The recovered solvents were distilled or condensed into a cold-trap under vacuum and collected in halogen-free or halogen-containing solvent containers, and stored for disposal.

Used NMR solvents were classified into halogen-free and halogen-containing solvents and were disposed as halogen containing wastes, respectively.

Drying agents such as $\mathrm{KOH}, \mathrm{CaCl}_{2}$ and $\mathrm{P}_{4} \mathrm{O}_{10}$ were hydrolyzed and disposed as acid or base wastes.

Whenever possible, sodium metal used for drying solvents was collected for recycling. The non-reusable sodium metal was carefully hydrolyzed in cold ethanol and poured into the basebath used for cleaning glassware.

Ethanol and acetone used for cold-baths (with solid $\mathrm{CO}_{2}$ or liquid $\mathrm{N}_{2}$ ) were subsequently used for cleaning glassware.

The acid-bath used for cleaning glassware was neutralized with $\mathrm{Na}_{2} \mathrm{CO}_{3}$ and the resulting $\mathrm{NaCl}$ solution was washed-off in the communal water drainage.

The residue of the base-bath used for glassware cleaning was poured into container for base wastes.

Amounts of various types of disposable wastes generated during the work:

Halogen-containing solvent wastes $4 \mathrm{~L}$

Halogen-free solvent wastes $40 \mathrm{~L}$

Acid wastes $20 \mathrm{~L}$

Base wastes $30 \mathrm{~L}$ 


\subsection{Abbreviations}

$\delta$

$\lambda$

$\mu$

$\tilde{v}$

av

C

calcd.

$\mathrm{Cp}$

$\mathrm{Cp}^{*}$

EI

eqv.

$\mathrm{eV}$

g

$\mathrm{h}$

$\mathrm{Hz}$

IR

$J$

K

L

M

$\mathrm{m} / \mathrm{z}$

$\mathrm{Mp}$

$\mathrm{M}^{+}$

$\mathrm{Me}$

Et

$i \operatorname{Pr}$

$t \mathrm{Bu}$

$\mathrm{Ph}$

$\mathrm{Ar}$

MS chemical shift

wavelength

bridging

wave number

average

Celsius

calculated

cyclopentadienyl

pentamethylcyclopetadienyl

electron impact ionization

equivalents

electron volt

grams

hours

Hertz

infrared

coupling constant

Kelvin

ligand

metal

mass/charge

melting point

molecular ion

methyl

ethyl

iso-propyl

tert-butyl

phenyl

aryl

mass spectrometry, mass spectra 
Chapter 9. Experimental Section

$\begin{array}{ll}\text { NMR } & \text { nuclear magnetic resonance } \\ \text { ppm } & \text { parts per million } \\ \text { q } & \text { quartet } \\ \text { R } & \text { organic substituents } \\ \text { S } & \text { singlet } \\ \text { d } & \text { doublet } \\ \text { t } & \text { triplet } \\ \text { sept } & \text { septet } \\ \text { m } & \text { multiplet } \\ \text { br } & \text { broad } \\ \text { THF } & \text { tetrahydrofuran }\end{array}$




\section{Reference:}

1. (a) Regitz, M. Angew. Chem. 1991, 103, 691 - 693; Angew. Chem., Int. Ed. Engl. 1991, 30, 674 - 676. (b) Herrmann, W. A.; Köcher, C. Angew. Chem. 1997, 109, 2256 - 2282; Angew. Chem., Int. Ed. Engl. 1997, 36, 2162 - 2187.

2. Arduengo, A. J.; Harlow, R. L.; Kline, M. J. Am. Chem. Soc. 1991, 113, 361 - 363.

3. Bourissou, D.; Guerret, O.; Gabbai, F. P.; Bertrand, G. Chem. Rev. 2000, 100, 39 - 91.

4. (a) Herrmann, W. A. Angew. Chem. 2002, 114, 1342 - 1363; Angew. Chem., Int. Ed. 2002, 41, 1290 - 1309. (b) Nair, V.; Bindu, S.; Sreekumar, V. Angew. Chem. 2004, 116, 5240 - 5245; Angew. Chem., Int. Ed. 2004, 43, 5130 - 5135.

5. (a) Matsunaga, P. T.; Kouvetakis, J.; Groy, T. L. Inorg. Chem. 1995, 34, 5103 - 5104.

(b) Fjeldberg, T.; Haaland, A.; Schilling, B. E. R.; Lappert, M. F.; Thorne, A. J. J. Chem. Soc., Dalton Trans. 1986, 1551 - 1556.

6. For examples, see: (a) du Mont, W. W.; Gust, T.; Seppälä, E.; Wismach, C. J. Organomet. Chem. 2004, 689, 1331 - 1336. (b) Rizzo, A.; Puzzarini, C.; Coriani, S.; Gauss, J. J. Chem. Phys. 2006, 124, 10 - 16. (c) Goldberg, N.; Ogden, J. S.; Almond, M. J.; Walsh, R.; Cannady, J. P.; Becerra, R.; Lee, J. A. Phys. Chem. Chem. Phys. 2003, 5, 5371 - 5377. (d) du Mont, W. W.; Gust, T.; Seppälä, E.; Wismach, C.; Jones, P. G.; Ernst, L.; Grunenberg, J.; Marsmann, H. C. Angew. Chem. 2002, 114, 3984 3986; Angew. Chem., Int. Ed. 2002, 41, 3829 - 3825. (e) Kang, S. H.; Han, J. S.; Lee, M. E.; Yoo, B. R.; Jung, I. N. Organometallics 2003, 22, 2551 - 2553. (f) Kang, S. H.; Han, J. S.; Yoo, B. R.; Lee, M. E.; Jung, I. N. Organometallics 2003, 22, 529 - 534.

7. Luke, B. T.; Pople, J. A.; Kroghjespersen, M. B.; Apeloig, Y.; Karni, M.; Chandrasekhar, J.; Schleyer, P. v. R. J. Am. Chem. Soc. 1986, 108, 270 - 284.

8. (a) Sasamori, T.; Tokitoh, N. Encyclopedia of Inorganic Chemistry II; King, R. B., Ed.; John Wiley \& Sons: Chichester, U.K., 2005; pp 1698-1740. (b) The Transition 
State: A Theoretical Approach; Fueno, T.; Ed.; Gordon and Breach Science Publishers: Langhorne, PA, 1999; pp 147-161.

9. Trinquier, G. J. Am. Chem. Soc. 1990, 112, 2130 - 2137.

10. (a) Weidenbruch, M. J. Organomet. Chem. 2002, 646, 39 - 52. (b) Weidenbruch, M. Organometallics 2003, 22, $4348-4360$.

11. West, R.; Fink, M. J.; Michl, J. Science 1981, 214, 1343 - 1344.

12. Brook, A. G.; Abdesaken, F.; Gutekunst, B.; Gutekunst. G.; Kallury, R. K. J. Chem. Soc., Chem. Commun. 1981, $191-192$.

13. (a) Okazaki, R,; West, R. Adv. Organomet. Chem. 1996, 39, 231 - 273. (b) Kira, M.; Iwamoto, T. Adv. Organomet. Chem. 2006, 54, 73 - 148. (c) Fukazawa, A.; Li, Y.; Yamaguchi, S.; Tsuji, H.; Tamao, K. J. Am. Chem. Soc. 2007, 129, 14164 - 14165. (d) Sasamori, T.; Yuasa, A.; Hosoi, Y.; Furukawa, Y.; Tokitoh, N. Organometallics 2008, $27,3325-3327$.

14. Hitchcock, P. B.; Lappert, M. F.; Miles, S. J.; Thorne, A. J. J. Chem. Soc., Chem. Commun. 1984, $480-482$.

15. Snow, J. T.; Murakami, S.; Masamune, S.; Williams, D. J. Tetrahedron Lett. 1984, 25, $4191-4194$

16. Pu, L.; Twamley, B.; Power, P. P. J. Am. Chem. Soc. 2000, 122, 3524 - 3525.

17. Stender, M.; Phillips, A. D.; Wright, R. J.; Power, P. P. Angew. Chem., Int. Ed. 2002, $41,1785-1787$.

18. Philips, A. D.; Wright, R. J.; Olmstead, M. M.; Power, P. P. J. Am. Chem. Soc. 2002, $124,5930-5931$.

19. Sekiguchi, A.; Kinjo, R.; Ichinohe, M. Science 2004, 305, 1755 - 1757.

20. (a) Jambor, R.; Kašná, B.; Kirschner, K. N.; Schürmann, M.; Jurkschat, K. Angew. Chem. 2008, 120, 1674 - 1677; Angew. Chem., Int. Ed. 2008, 47, 1650 - 1653. (b) Green, S. P.; Jones, C.; Junk, P. C.; Lippert, K. -A.; Stasch, A. Chem. Commun. 2006, 
3978 - 3980. (c) Wang, W.; Inoue, S.; Yao, S.; Driess, M. Chem. Commun. 2009, 2661 - 2663. (d) Lee, V. Y.; Sekiguchi, A. Organometallics 2004, 23, 2822 - 2834. (e) Weidenbruch, M. Angew. Chem. 2003, 115, 2322 - 2324; Angew. Chem., Int. Ed. 2003, 42, 2222 - 2224. (f) Sekiguchi, A.; Lee, V. Y. Chem. Rev. 2003, 103, 1429 1448. (g) West, R. Polyhedron 2002, 21, 467 - 472. (h) Kira, M. Pure Appl. Chem. 2000, 72, 2333 - 2342. (i) Bejan, I.; Scheschkewitz, D. Angew. Chem. 2007, 119, 5885 - 5888; Angew. Chem. Int. Ed. 2007, 46, 5783 - 5786. (j) Sekuguchi, A.; Inoue, S.; Ichinohe, M.; Arai, Y. J. Am. Chem. Soc. 2004, 126, 9626 - 9629. (k) Scheschkewitz, D. Chem. Eur. J. 2009, 15, 2476 - 2485. (1) Sasamori, T.; Hironaka, K.; Sugiyama, Y.; Takagi, N.; Nagase, S.; Furukawa, Y.; Tokitoh, N. J. Am. Chem. Soc. 2008, 130, 13856 - 13857. (m) Sugijama, Y.; Sasamori, T.; Hosoi, Y.; Furukawa, Y.; Takagi, N.; Nagase, S.;Tokitoh, N. J. Am. Chem. Soc. 2006, 128, 1023 - 1031. (n) Mizuhata, Y.; Sasamori, T.; Tokitoh, N. Chem. Rev. 2009, 109, 3479 - 3511.

21. Wang, Y.; Xie, Y.; Wei, P.; King, R. B.; Schaefer, H. F., III; Schleyer, P. v. R.; Robinson, G. H. Science 2008, 321, 1069 - 1071.

22. Denk, M.; Lennon, R.; Hayashi, R.; West, R.; Belyakov, A. V.; Verne, H. P.; Haaland, A.; Wagner, M.; Metzler, N. J. Am. Chem. Soc. 1994, 116, 2691 - 2692.

23. (a) Gehrhus, B.; Lappert, M. F.; Heinicke, J.; Boese, R.; Bläser, D. J. Chem. Soc., Chem. Commun. 1995, 1931 - 1932. (b) Kira, M.; Ishida, S.; Iwamoto, T.; Kabuto, C. J. Am. Chem. Soc. 1999, 121, 9722 - 9723. (c) Driess, M.; Yao, S.; Brym, M.; van Wüllen, C.; Lentz, D. J. Am. Chem. Soc. 2006, 128, 9628 - 9629.

24. (a) Li, R. -E.; Sheu, J. -H.; Su, M. -D. Inorg. Chem. 2007, 46, 9245 - 9253. (b) Bharatam, P. V.; Moudgil, R.; Kaur, D. Inorg. Chem. 2003, 42, 4743 - 4749. (c) Bharatam, P. V.; Moudgil, R.; Kaur, D. Organometallics 2002, 21, 3683 - 3690.

25. (a) So, C. -W.; Roesky, H. W.; Magull, J.; Oswald, R. B. Angew. Chem. 2006, 118, 4052 - 4054; Angew. Chem., Int. Ed. 2006, 45, 3948 - 3950. (b) Ghadwal, R. S.; 
Roesky, H. W.; Merkel, S.; Henn, J.; Stalke, D. Angew. Chem. 2009, 121, 5793 5796; Angew. Chem., Int. Ed. 2009, 48, 5683 - 5686.

26. (a) Schmeisser, M.; Voss, P. Z. Anorg. Allg. Chem. 1964, 334, 50 - 56. (b) Timms, P. L. Inorg. Chem. 1968, 7, 387 - 389. (c) Timms, P. L. Acc. Chem. Res. 1973, 6, 118 123.

27. Wang, Y.; Robinson, G. H. Chem. Commun. 2009, 5201 - 5213.

28. (a) Cui, C.; Roesky, H. W.; Schmidt, H.-G.; Noltemeyer, M.; Hao, H.; Cimpoesu, F. Angew. Chem. 2000, 112, 4444 - 4446; Angew. Chem., Int. Ed. 2000, 39, 4274 4276. (b) Ding, Y.; Roesky, H. W.; Noltemeyer, M.; Schmidt, H.-G.; Power, P. P. Organometallics 2001, 20, 1190 - 1194.

29. (a) Jutzi, P.; Kanne, D.; Krüger, C. Angew. Chem. 1986, 98, 163 - 164; Angew. Chem., Int. Ed. Engl 1986, 25, 164. (b) Resa, I.; Carmona, E.; Puebla, E. G.; Monge, A. Science 2004, 305, $1136-1138$.

30. Nguyen, T.; Sutton, A. D.; Brynda, M.; Fettinger, J. C.; Long, G. J.; Power, P. P. Science 2005, 310, $844-847$.

31. Green, S. P.; Jones, C.; Stasch, A. Science 2008, 318, 1754 - 1757.

32. (a) Wang, Y.; Quillian, B.; Wei, P.; Wannere, C. S.; Xie, Y.; King, R. B.; Schaefer, H. F., III; Schleyer, P. v. R.; Robinson, G. H. J. Am. Chem. Soc. 2007, 129, 12412 12413. (b) Wang, Y.; Quillian, B.; Wei, P.; Xie, Y.; Wannere, C. S.; King, R. B.; Schaefer, H. F., III; Schleyer, P. v. R.; Robinson, G. H. J. Am. Chem. Soc. 2008, 130, 3298 - 3299. (c) Wang, Y.; Xie, Y.; Wei, P.; King, R. B.; Schaefer, H. F., III; Schleyer, P. v. R.; Robinson, G. H. J. Am. Chem. Soc. 2008, 130, 14970 - 14971. (d) Abraham, M. Y.; Wang, Y.; Xie, Y.; Wei, P.; Schaefer, H. F., III; Schleyer, P. v. R.; Robinson, G. H. Chem. Eur. J. 2010, 16, 432 - 435. (e) Quillian,B.;Wei, P.;Wannere,C. S.; Schleyer, P. v. R.; Robinson, G. H. J. Am. Chem. Soc. 2009, 131, $3168-3169$. 
33. Gerhardt, C. Annalen 1858, 108, 219.

34. (a) Hao, S.; Gambarotta, S.; Bensimon, C.; Edema, J. J. H. Inorg. Chim. Acta 1993, 213, 65 - 74. (b) Coles, M. P.; Swenson, D. C.; Jordan, R. F.; Young Jr., V. G. Organometallics 1997, 16, 5183 - 5194. (c) Barker, J.; Kilner, M. Coord. Chem. Rev. 1994, 133, $219-300$.

35. Cowley, A. H. J. Organomet. Chem. 2004, 689, 3866 - 3872.

36. Mandal, S. K.; Roesky, H. W. Chem. Commun. 2010, DOI: 10.1039/c0cc01003k

37. So, C.-W.; Roesky, H. W.; Gurubasavaraj, P. M.; Oswald, R. B.; Gamer, M. T.; Jones, P. T.; Blaurock, S. J. Am. Chem. Soc. 2007, 129, 12049 - 12054.

38. Jana, A.; Objartel, I.; Roesky, H. W.; Stalke, D. Inorg. Chem, 2009, 48, 798 - 800.

39. Cui, H.; Shao, Y.; Li, X; Kong, L.; Cui, C. Organometallics 2009, 28, 5191 - 5195.

40. Benkeser, R. A. Acc. Chem. Res. 1971, 4, $94-100$.

41. Driess, M.; Yao, S. L.; Brym, M.; van Wüllen, C. Angew. Chem. 2006, 118, 44554458; Angew. Chem., Int. Ed. 2006, 45, 4349-4352.

42. (a) Barton, T. J. in Comprehensive Organometallic Chemistry; Wilkinson, G., Stone, F. G. A., and Abel, E. W., Eds.; Pergamon Press: Oxford, U.K., 1982; Vol. 2, p 205. (b) Aylett, B. J., and Sullivan, A. C. In Comprehensive Organometallic Chemistry II; Wilkinson, G., Stone, F. G. A., and Abel, E. W., Eds.; Pergamon Press: Oxford, U.K., 1995; Vol. 2, p 45.

43. (a) Ando, W.; Shiba, T.; Hidaka, T.; Morihashi, K.; Kikuchi, O. J. Am. Chem. Soc. 1997, 119, 3629 - 3630. (b) Metz, S.; Burschka, C.; Tacke, R. Organometallics 2008, 27, 6032 - 6034. (c) Seiler, O.; Büttner, M.; Penka, M.; Tacke, R. Organometallics 2005, 24, 6059 - 6062. (d) Tacke, R.; Burschka, C.; Richter, I.; Wagner, B.; Willeke, R. J. Am. Chem. Soc. 2000, 122, 8480 - 8485. (e) Tacke, R.; Ulmer, B.; Wagner, B.; Arlt, M. Organometallics 2000, 19, 5297 - 5309. (f) Theis, B.; Metz, S.; Burschka, C.; Bertermann, R.; Maisch, S.; Tacke, R. Chem. Eur. J. 2009, 15, 7329 - 7338; (g) 
Seiler, O.; Burschka, C.; Metz, S.; Penka, M.; Tacke, R. Chem. Eur. J. 2005, 11, 7379 $-7386$.

44. (a) Kottke, T.; Stalke, D. J. Appl. Crystallogr. 1993 26, 615 - 619. (b) Sheldrick, G. M. Acta Crystallogr. Sect. A 2008, 64, 112 - 122. (c) Stalke, D. Chem. Soc. Rev. 1998, 27, 171 - 178. (d) Schulz, T.; Meindl, K.; Leusser, D.; Stern, D.; Ruf, M.; Sheldrick, G. M.; Stalke, D. J. Appl. Crystallogr. 2009, 42, 885 - 891.

45. (a) Weidenbruch, M. in The Chemistry of Organic Silicon Compounds, Rappoport, Z.; Apeloig, Y. Eds. ( Wiley, Chichester, UK, 2001), vol. 3, chap. 5.

46. (a) Cowley, A. H.; Ebsworth, E. A. V.; Mehrotra, S. K.; Rankin, M. D. Walkinshaw, D. W. H. J. Chem. Soc., Chem. Commun. 1982, 1099 - 1100; (b) 'Tables of Interatomic Distances and Configurations in Molecules and Ions,' Ed.; Sutton, L. E. Chem Soc. Spec. Publ. 1965, 18, 5225.

47. For example: (a) Batcheller, S. A.; Masamune, S. Tetrahedron Lett.1988, 29, 3382 3384. (b) Ando, W.; Tsumuraya, T. J. Chem. Soc., Chem. Commun.1989, $770-772$. (c) Apeloig, Y.; Bravo-Zhivotovskii, D.; Zharov, I.; Panov, V.; Leigh, W. J.; Sluggett, G. W. J. Am. Chem. Soc. 1998, 120, 1398 - 1404. (d) Bravo- Zhivotovskii, D.; Zharov, I.; Kapon, M.; Apeloig, Y. J. Chem. Soc., Chem. Commun. 1995, 1625 -1626. (e) Fukaya, N.; Ichinohe, M.; Kabe, Y.; Sekiguchi, A. Organometallics 2001, 20, 3364 - 3366. (f) Fukaya, N.; Ichinohe, M.; Sekiguchi, A. Angew. Chem. 2000, 112, 4039 4042; Angew. Chem., Int. Ed. 2000, 39, 3881 - 3884. (g) Cui, C.; Olmstead, M. M.; Power, P. P. J. Am. Chem. Soc. 2004, 126, 5062 - 5063.

48. Bourissou, D.; Guerret, O.; Gabbai, F. P.; Bertrand, G. Chem. Rev. 2000, 100, 39 - 91.

49. Weidenbruch, M.; Olthoff, S.; Saak, W.; Marsmann, H. Eur. J. Inorg. Chem. 1998, $1755-1758$.

50. (a) Minkin, V. I. ; Glukhovtsev, M. N.; Simkin, B. Y. Aromaticity and Antiaromaticity—Electronic and Structural Aspects, J. Wiley \& Sons, New York, 
1994. (b) Cyranski, M. K. Chem. Rev. 2005, 105, 3773 - 3811. (c) Kaufman, H. S.; Fankuchen, I.; Mark, H. Nature 1948, 161, 165. (d) Thomas, P. M.; Weber, A. J. Raman Spectros. 1978, 7, 353 - 357. (e) Bastiansen, O.; Hassel, O.; Langseth, A. Nature 1947, 160, 128.

51. (a) Evans, W. J.; Clark, R. D.; Ansari, M. A.; Ziller, J. W. J. Am. Chem. Soc. 1998, 120, 9555 - 9563. (b) Evans, W. J.; Nyce, G. W.; Ziller, J. W. Angew. Chem. 2000, 112, 246 - 248; Angew. Chem., Int. Ed. 2000, 39, 240 - 242. (c) Cummins, C. C.; Diaconescu, P. L. J. Am. Chem. Soc. 2002, 124, 7660 - 7661. (d) Summerscales, O. T.; Jones, S. C.; Cloke, F. G. N.; Hitchcock, P. B. Organometallics 2009, 28, 5896 5908. (e) Hu, N.; Gong, L.; Jin, Z.; Chen, W. J. Organomet. Chem. 1988, 352, 61 66. (f) Walter, M. D.; Wolmershäuser, G.; Sitzmann, H. J. Am. Chem. Soc. 2005, 127, 17494 - 17503. (g) Summerscales, O: T.; Wang, X.; Power, P. P. Angew. Chem. 2010, 122, 4898 - 4890; Angew. Chem., Int. Ed. 2010, 49, 4788 - 4790.

52. (a) Bulo, R. E.; Jansen, H.; Ehlers, A. W.; de Kanter, F. J. J.; Schakel, M.; Lutz, M.; Spek, A. L.; Lammertsma, K. Angew. Chem. 2004, 116, 732 - 735; Angew. Chem., Int. Ed. 2004, 43, 714 - 717. (b) Bulo, R. E.; Allaart, F.; Ehlers, A.W.; de Kanter, F. J. J. ; Schakel, M.; Lutz, M.; Spek, A. L.; Lammertsma, K. J. Am. Chem. Soc. 2006, $128,12169-12173$.

53. (a) Fink, M. J.; De Young, D. J.; West, R.; Michl, J. J. Am. Chem. Soc. 1983, 105, 1070 - 1071. (b) Fink, M. J.; Haller, K. J.; West, R.; Michl, J. J. Am. Chem. Soc. 1984, 106, 822 - 823. (c) Michalczyk, M. J.; Fink, M. J.; Haller, K. J.; West, R.; Michl, J. Organometallics 1986, 5, 531 - 536. (d) Sohn, H.; Tan, R. P.; Powell, D. R.; West, R. Organometallics 1994, 13, 1390 - 1394.

54. (a) Kipping, F. S.; Lloyd, L. L. J. Chem. Soc., Trans. 1901, 449 - 459. (b) Greenwood, N. N.; Earnshaw, A. Chemistry of the Elements (2nd Ed.), Oxford: ButterworthHeinemann, p. 362. (c) Corey, J. Y.; Historical overview and comparison of silicon 
with carbon, pp. 1-56 in Patai, S. and Rappoport, Z. Eds. The Chemistry of Organic Silicon Compounds (New York: Wiley, 1989).

55. (a) Xiong, Y.; Yao, S.; Driess, M. J. Am. Chem. Soc. 2009, 131, 7562 - 7563. (b) Xiong, Y.; Yao, S.; Müller, R.; Kaupp, M.; Driess, M. J. Am. Chem. Soc. 2010, 132, $6912-6913$

56. (a) Haaf, M.; Schmedake, T. A.; West, R. Acc. Chem. Res. 2000, 33, 704 - 714. (b) Jutzi, P.; Möhrke, A. Angew. Chem. 1989, 101, 769 - 770; Angew. Chem., Int. Ed. Engl. 1989, 28, 762 - 763. (c) Suzuki, H.; Tokitoh, N.; Nagase, S.; Okazaki, R. J. Am. Chem. Soc. 1994, 116, 11578 - 11579. (d) Jutzi, P.; Eikenberg, D.; Möhrke, A.; Neumann, B.; Stammler, H.-G. Organometallics 1996, 15, 753 - 759.

57. (a) Regitz, M.; Binger, P. in Multiple Bonds and Low Coordination in Phosphorus Chemistry; Regitz, M.; Scherer, O.; Eds.; Thieme: Sttugart, 1990; p 58 - 93. (b) Nguyen, M. T.; Landuyt, L.; Vanquickenborne, L. G. J. Org. Chem. 1993, 58, 2817 2821. (c) Figueroa, J. S.; Cummins, C. C. J. Am. Chem. Soc. 2004, 126, 13916 13917. (d) Figueroa, J. S.; Cummins, C. C. Dalton Trans. 2006, 2161 - 2168. (e) West, N. M.; White, P. S.; Templeton, J. L.; Nixon, J. F. Organometallics 2009, 28, $1425-1434$.

58. (a) Bender, H. R. G.; Niecke, E.; Nieger, M. J. Am. Chem. Soc. 1993, 115, 3314 3315. (b) Rademacher, P. in Grösse und Gestalt von Molekulen, Strukturen organischer Molekiile. Physikalische Organische Chemie; Klessinger, M., Ed.; Verlag Chemie: Weinheim, 1987; p 55.

59. (a) Kaftory, M.; Kapon, M.; Botoshansky, M. in The Chemistry of Organic Silicon Compounds; Rappoport, Z. Apeloig, Y. Eds.; Wiley: Chichester, U.K., 1998; Vol. 2, Chapter 5.

60. Kinjo, R.; Ichinohe, M.; Sekiguchi, A.; Takagi, N.; Sumimoto, M.; Nagase, S. J. Am. Chem. Soc. 2007, 129, $7766-7767$. 
61. Kramkowski, P.; Scheer, M. Angew. Chem. 2000, 112, 959 - 962; Angew. Chem., Int. Ed. 2000, 39, 928 - 931.

62. (a) Nolan, S. P. in N-Heterocyclic Carbenes in Synthesis; Ed.; Wiley-VCH: Weinheim, Germany, 2006. (b) Glorius, F. in N-Heterocyclic Carbenes in Transition Metal Catalysis; Ed.; Springer-Verlag: Berlin, 2007. (c) Clavier, H.; Nolan, S. P. Annu. Rep. Prog. Chem., Sect B: Org. Chem. 2007, 103, 193 - 222. (d) Weskamp, T.; Böcher, V. P. W.; Herrmann, W. A. J. Organomet. Chem. 2000, 600, 12 - 22. (e) Jafarpour, L.; Nolan, S. P. Adv.Organomet. Chem. 2000, 46, 181 - 222. (f) Harrold, N. D.; Waterman, R.; Hillhouse, G. L.; Cundari, T. R. J. Am. Chem. Soc. 2009, 131, 12872 - 12873. (g) Sinha, A.; Rahaman, S. M. W.; Sarkar, M.; Saha, B.; Daw, P.; Bera, J. K. Inorg. Chem. 2009, 48, 11114 - 11122. (h) Lee, Y.; Li, B.; Hoveyda, A. H. J. Am. Chem. Soc. 2009, 131, 11625 - 11633.

63. (a) Catalyzed Direct Reactions of Silicon; Lewis, K. M.; Rethwisch, D. G.; Eds.; Elsevier: Amsterdam, The Netherlands, 1993. (b) Sharma, H. K.; Pannell, K. H. Chem. Rev. 1995, 95, 1351 - 1374. (c) Curtis, M. D.; Epstein, P. S. Adv. Organomet. Chem. 1981, 19, 213 - 232. (d) Gauvin, F.; Harrod, J. F.; Woo, H. G. Adv. Organomet. Chem. 1998, 42, 363 - 405. (e) Corey, J. Y. Adv. Organomet. Chem. 2004, 51, 1 - 52. (f) Tilley, T. D. Comments Inorg. Chem. 1990, 10, 37 - 46. (g) Fürstner, A.; Krause, H.; Lehmann, C. W. Chem. Commun. 2001, 2372 - 2373. (h) Calimano, E.; Tilley, T. D. J. Am. Chem. Soc. 2009, 131, 11161 - 11173. (i) Prévost, M.; Woerpel, K. A. J. Am. Chem. Soc. 2009, 131, 14182 - 14183. (j) Ohmura, T.; Masuda, K.; Takase, I.; Suginome, M. J. Am. Chem. Soc. 2009, 131, 16624 - 16625.

64. (a) Zybill, C.; Müller, G. Angew. Chem. 1987, 99, 683 - 684. Angew. Chem., Int. Ed. Engl. 1987, 26, 669 - 670. (b) Straus, D. A.; Tilley, T. D.; Rheingold, A. L.; Geib, S. J. J. Am. Chem. Soc. 1987, 109, 5872 - 5873. 
65. (a) Gehrhus, B.; Hitchcock, P. B.; Lappert, M. F.; Heinicke, J.; Boese, R.; Bläser, D. J. Organomet. Chem. 1996, 521, 211 - 220. (b) Haaf, M.; Schmiedl, A.; Schmedake, T. A.; Powell, D. R.; Millevolte, A. J.; Denk, M.; West, R. J. Am. Chem. Soc. 1998, 120, 12714 - 12719. (c) Metzler, N.; Denk, M. Chem. Commun. 1996, 2657 - 2658. (d) Denk, M.; Hatano, K.; Lough, A. J. Eur. J. Inorg. Chem. 1998, 1067 - 1070. (e) Drost, C.; Gehrhus, B.; Hitchcock, P. B.; Lappert, M. F. Chem. Commun. 1997, 1845 - 1846. (f) Schmedake, T. A.; Haaf, M.; Apeloig, Y.; Müller, T.; Bukalov, S.; West, R. J. Am. Chem. Soc. 1999, 121, 9479 - 9480. (g) Boesveld, W. M.; Gehrhus, B.; Hitchcock, P. B.; Lappert, M. F.; Schleyer, P. R. Chem. Commun. 2000, 955 - 956. (h) Gehrhus, B.; Hitchcock, P. B.; Lappert, M. F. Organometallics 1997, 16, 4861 - 4864. (i) Gehrhus, B.; Hitchcock, P. B.; Lappert, M. F. Organometallics 1998, 17, 1378 - 1382. (j) Gehrhus, B.; Lappert, M. Polyhedron 1998, 17, 999-1000. (k) Denk, M.; Hayashi, R.; West, R. J. Am. Chem. Soc. 1994, 116, 10813 - 10814. (1) Schmedake, T. A.; Haaf, M.; Paradise, B. J.; Millevolte, A. J.; Powell, D. R.; West, R. J. Organomet. Chem. 2001, 636, 17 - 25. (m) Epping, D. J; Yao, S.; Karni, M.; Apeloig, Y.; Driess, M. J. Am. Chem. Soc. 2010, 132, 5443 - 5455. (n) Xiong, Y.; Yao, S.; Brym, M.; Driess, M. Angew. Chem. 2007, 119, 4595 - 4597; Angew. Chem., Int. Ed. 2007, 46, 4511 4513. (o) Yao, S.; van Wüllen, C.; Sun, X.-Y.; Driess, M. Angew. Chem. 2008, 120, 3294 - 3297; Angew. Chem., Int. Ed. 2008, 47, 3250 - 3253. (p) Yao S.; Xiong. Y.; van Wüllen, C.; Driess. M. Organometallics, 2009, 28, 1610 - 1612. (q) Xiong. Y.; Yao S.; Driess. M. Organometallics 2009, 28, 1927 - 1933. (r) Jana, A.; Roesky, H. W.; Schulzke, C.; Samuel, P. P. Organometallics 2009, 28, 6574 - 6577. (s) Jana, A.; Schulzke, C.; Roesky, H. W. J. Am. Chem. Soc. 2009, 131, 4600 - 4601. (t) Xiong. Y.; Yao S.; Driess. M. Organometallics 2010, 29, 987 - 990. (u) Xiong, Y.; Yao, S.; Driess, M. J. Am. Chem. Soc. 2009, 131, 7562 - 7563. (v) Xiong, Y.; Yao, S.; Driess, M. Chem. Eur. J. 2009, 15, 5545 - 5551. (w) Nagendran, S.; Roesky, H. W. 
Organometallics 2008, 27, 457 - 492. (x) Mizuhata, Y.; Sasamori, T.; Tokitoh, N. Chem. Rev. 2009, 109, 3479 - 3511.

66. Braga, D.; Grepioni, F.; Orpen, A. G. Organometallics 1993, 12, 1481 - 1483.

67. Haaf, M.; Hayashi, R.; West, R. J. Chem. Soc., Chem. Commun. 1994, 33 - 34.

68. (a) Feldman, J. D.; Mitchell, G. P.; Nolte, J. -O.; Tilley, T. D. J. Am. Chem. Soc. 1998, 120, 11184 - 11185. (b) Gehrhus, B.; Hitchcock, P. B.; Lappert, M. F.; Maciejewski, H. Organometallics 1998, 17, 5599 - 5601. (c) Petri, S. H. A.; Eikenberg, D.; Neumann, B.; Stammler, H.-G.; Jutzi, P. Organometallics 1999, 18, 2615 - 2618. (d) Schmedake, T. A.; Haaf, M.; Paradise, B. J.; Powell, D.; West, R. Organometallics 2000, 19, 3263 - 3265. (e) Dysard, J. M.; Tilley, T. D. Organometallics 2000, 19, 4726 - 4732. (f) Clendenning, S. B.; Gehrhus, B.; Hitchcock, P. B.; Moser, D. F.; Nixon, J. F.; West, R. J. Chem. Soc., Dalton Trans. 2002, 484 - 490. (g) Amoroso, D.; Haaf, M.; Yap, G. P. A.; West, R.; Fogg, D. E. Organometallics 2002, 21, 534 - 540. (h) Avent, A. G.; Gehrhus, B.; Hitchcock, P. B.; Lappert, M. F.; Maciejewski, H. J. Organomet. Chem. 2003, 686, $321-331$.

69. Yang, W.; Fu, H.; Wang, H.; Chen, M.; Ding, Y.; Roesky, H. W.; Jana, A. Inorg. Chem. 2009, 48, $2058-2060$.

70. Li, J.; Merkel, S.; Henn, J.; Meindl, K.; Döring, A.; Roesky, H. W.; Ghadwal, R. S.; Stalke, D. Inorg. Chem. 2010, 49, 775 - 777.

71. Meltzer, A.; Präsang, C.; Driess, M. J. Am. Chem. Soc. 2009, 131, 7232 - 7233.

72. Apeloig, Y.; Pauncz, R.; Karni, M.; West, R.; Steiner, W.; Chapman, D. Organometallics 2003, 22, $3250-3256$.

73. Experimental determinations of $\Delta E \mathrm{ST}$ in $\mathrm{SiH}_{2}$ : (a) Kasden, A.; Herbst, E.; Lineberger, W. C. J. Chem. Phys. 1975, 62, 541 - 548. (b) Berkowitz, J. L.; Green, J. P.; Cho, H.; Ruščić, R. J. Chem. Phys. 1987, 86, 1235 - 1248. 
74. (a) Carter, E. A.; Goddard, W. A. III; J. Phys.Chem., 1986, 90, 998 - 1001; (b) Trinquier, G.; Malrieu, J.-P. J. Am. Chem. Soc. 1987, 109, 5303 - 5315; c) Malrieu, J.P.; Trinquier, G. J. Am. Chem. Soc. 1989, 111, 5916 - 5921. (c) Driess, M.; Grützmacher, H. Angew. Chem. 1996, 108, 900 - 929; Angew. Chem., Int. Ed. Engl. 1996, 35, 828 - 856; (d) Grützmacher, H.; Fässler, T. F. Chem. Eur. J. 2000, 6, 2317 -2325 .

75. Wiberg, N.; Vasisht, S. K.; Fischer, G.; Mayer, P. Z. Anorg. Allg. Chem. 2004, 630, $1823-1828$.

76. Corey, J. Y. in The Chemistry of Organic Silicon Compounds, Volume 1, Patai, S. Rappoport, Z. Eds. (Wiley, Chichester, UK, 1989), chap. 1.

77. Nesper, R.; Currao, A.; Wengert, S. in Organosilicon Chemistry II: From Molecules to Materials, Auner, N. Weis, J. Eds. (VCH, Weinheim, Germany, 1996), p. 469.

78. Weidenbruch, M. in The Chemistry of Organic Silicon Compounds, Volume 3, Rappoport, Z.; Apeloig, Y. Eds. (Wiley, Chichester, UK, 2001), chap. 5.

79. Cowley, A. H.; White, W. D.; Damasco, M. C. J. Am. Chem. Soc. 1969, 91, 1922 1928.

80. (a) Lee, C. Yang, W.; Parr, R. G. Phys. Rev. B 1988, 37, 785 - 789. (b) Miehlich, B.; Savin, A.; Stoll, H.; Preuss, H. Chem. Phys. Lett. 1989, 157, 200 - 206. (c) Becke, A. D. J. Chem. Phys. 1993, 98, $5648-5652$.

81. (a) Hay, P. J.; Wadt, W. R. J. Chem. Phys. 1985, 82, 270 - 283. (b) Wadt, W. R.; Hay, P. J. J. Chem. Phys. 1985, 82, 284 - 298. (c) Hay, P. J.; Wadt, W. R. J. Chem. Phys. 1985, 82, $299-310$.

82. Gaussian 03 (Revision C.02): Frisch, M. J.; Trucks, G. W.; Schlegel, H. B.; Scuseria, G. E.; Robb, M. A.; Cheeseman, J. R.; Montgomery, Jr., J. A.; Vreven, T.; Kudin, K. N.; Burant, J. C.; Millam, J. M.; Iyengar, S. S.; Tomasi, J.; Barone, V.; Mennucci, B.; Cossi, M.; Scalmani, G.; Rega, N.; Petersson, G. A.; Nakatsuji, H.; Hada, M.; Ehara, 
M.; Toyota, K.; Fukuda, R.; Hasegawa, J.; Ishida, M.; Nakajima, T.; Honda, Y.; Kitao, O.; Nakai, H.; Klene, M.; Li, X.; Knox, J. E.; Hratchian, H. P.; Cross, J. B.; Bakken, V.; Adamo, C.; Jaramillo, J.; Gomperts, R.; Stratmann, R. E.; Yazyev, O.; Austin, A. J.; Cammi, R.; Pomelli, C.; Ochterski, J. W.; Ayala, P. Y.; Morokuma, K.; Voth, G. A.; Salvador, P.; Dannenberg, J. J.; Zakrzewski, V. G.; Dapprich, S.; Daniels, A. D.; Strain, M. C.; Farkas, O.; Malick, D. K.; Rabuck, A. D.; Raghavachari, K.; Foresman, J. B.; Ortiz, J. V.; Cui, Q.; Baboul, A. G.; Clifford, S.; Cioslowski, J.; Stefanov, B. B.; Liu, G.; Liashenko, A.; Piskorz, P.; Komaromi, I.; Martin, R. L.; Fox, D. J.; Keith, T.; Al-Laham, M. A.; Peng, C. Y.; Nanayakkara, A.; Challacombe, M.; Gill, P. M. W.; Johnson, B.; Chen, W.; Wong, M. W.; Gonzalez, C.; and Pople, J. A.; Gaussian, Inc., Wallingford CT, 2004.

83. (a) Noodleman, L. J. Chem. Phys. 1981, 74, 5737 - 5743. (b) Sarkar, S.; Dutta, A.; Mondal, A.; Chopra, D.; Ribas, J.; Rajak, K. K.; Sairam, S. M.; Pati, S. K. J. Phys. Chem. B 2006, 110, 12 - 15. (c) Behera, J. N.; Sundaresan, A.; Pati, S. K.; Rao C. N. R. ChemPhysChem 2007, 8, 217 - 219.

84. (a) Ichinohe, M.; Igarashi, M.; Sanuki, K.; Sekiguchi, A. J. Am. Chem. Soc. 2005, 127, 9978 - 9979. (b) Lee, V. Y.; Yasuda, H.; Sekiguchi, A. J. Am. Chem. Soc. 2007, 129, 2436 - 2437. (c) Lee, V. Y.; Miyazaki, S.; Yasuda, H.; Sekiguchi, A. J. Am. Chem. Soc. 2008, 130, 2758 - 2759. (d) Takeuchi, K.; Ichinohe, M.; Sekiguchi, A.; Guo, J. D.; Nagase, S. Organonmetallics 2009, 28, 2658 - 2660.

85. Malatesta, V.; Ingold, K. U. J. Am. Chem. Soc. 1981, 103, 609 - 614.

86. Yeong, H. -X.; Lau, K. -C.; Xi, H. -W.; Lim, K. H.; So, C.-W. Inorg. Chem. 2010, $49,371-373$.

87. (a) Stalke, D.; Wedler, M.; Edelmann, F. T. J. Organomet. Chem. 1992, 431, C1 - C5.

(b) Wedler, M.; Knösel, F.; Pieper, U.; Stalke, D.; Edelmann, F. T.; Amberger, H.-D. Chem. Ber. 1992, 125, $2171-2181$. 
88. (a) Kudo, T.; Nagase, S. J. Am. Chem. Soc. 1985, 107, 2589 - 2595. (b) Bacharach, S. M.; Streitwieser, A. J. Am. Chem. Soc. 1985, 107, 1186 - 1190.

89. Yao, S.; Xiong, Y.; Brym, M.; Driess, M. J. Am. Chem. Soc. 2007, 129, 7268 - 7269.

90. (a) Basolo, F.; Johnson, R. C. Coordination Chemistry, The Benjamin/Cummings Publishing Company, London, 1964, p. 130. (b) Omae, I. Coord. Chem. Rev. 2004, $248,995-1023$.

91. (a) Wade, L.G. (Sixth Ed., 2006). Organic Chemistry. Pearson Prentice Hall. pp. 279.

(b) Moss, G. P.; Smith, P. A. S.; Tavernier, D. Pure and Applied Chemistry, 1995 67, $1307-1375$.

92. Hoffmann, R. Angew. Chem. 1982, 94, 725 - 739; Angew. Chem., Int. Ed. 1982, 21, $711-724$.

93. (a) Scherer, O. J.; Sitzmann, H.; Wolmershäuser, G. Angew. Chem. 1985, 97, 352 354; Angew. Chem., Int. Ed. Engl. 1985, 24, 351 - 353. (b) Scherer, O. J.; Brück, T. Angew. Chem. 1987, 99, 59; Angew. Chem., Int. Ed. Engl. 1987, 26, 59.

94. The analogy between hydrocarbon species and organophosphorus compounds has been described: Dillon, K. B.; Mathey, F.; Nixon, J. F. Phosphorus: The Carbon Copy; Wiley: Chichester, U.K., 1998.

95. Cossairt, B. M.; Piro, N. A.; Cummins, C. C. Chem. Rev. 2010, 110, $4164-4177$

96. Caporali, M.; Gonsalvi, L.; Rossin, A.; Peruzzini, M. Chem. Rev. 2010, 110, 4178 4235 .

97. Scheer, M.; Balazs, G.; A. Seitz, Chem. Rev. 2010, 110, 4236 - 4256.

98. Xiong, Y.; Yao, S.; Bill, E.; Driess, M. Inorg. Chem. 2009, 48, 7522 - 7524.

99. (a) Faraday, M. Philos. Trans. R. Soc. London., 1825, 440 - 464. (b) Kekulé, A. Justus Liebigs Ann. Chem. 1866, 137, 129 - 136. (c) Carey, F. A.; Sundberg, R. J. Advanced Organic Chemistry, Part A: Structure and Mechanisms, 4th Ed. Kluwer Academic/Plenum Publishers, New York, 2000; (d) Schleyer, P. v. R. Chem. Rev. 
2001, 101, 1115 - 1118; and the thematic issue Aromaticity (e) Schleyer, P. v. R. Chem. Rev. 2005, 105, 3433 - 3435; and the thematic issue delocalization-pi and sigma; (f) Matito, E.; Poater, J.; Sola, M.; Schleyer, P. v. R. Chem. Reactivity Theory, CRC Press, 2009, $419-438$.

100. (a) Stock, A.; Pohland. E. Chem. Ber., 1926, 59B, 2215 - 2223. (b) Islas, R.; Chamorro, E.; Robles, J.; Heine, T.; Santos, J. C.; Merino, G. Struct. Chem. 2007, 18, 833 - 839. (c) Ashe, A. J. III; Kampf, J. W.; Nakadira, Y.; Pace, J. M. Angew. Chem. 1992, 104, 1267 - 1269; Angew. Chem., Int. Ed. Engl. 1992, 31, 1255 - 1258. (d) Ashe, A. J. III; Kampf, J. W.; Waas, J. R. Organometallics 1995, 14, 3141 - 3142. (e) Hofmann, M.; Berndt, A. Heteroatom. Chem. 2006, 17, 24 - 37. (f) Braunschweig, H.; Chiu, C.-W.; Radacki, K.; Kupfer, T. Angew. Chem. 2010, 122, 2085 - 2088; Angew. Chem., Int. Ed. 2010, 49, 2041 - 2044.

101. (a) Heinemann, C.; Müller, T.; Apeloig, Y.; Schwarz, H. J. Am. Chem. Soc. 1996, 118, 2023 - 2038. (b) Tokitoh, N.; Wakita, K.; Okazaki, R.; Nagase, S.; Schleyer, P. v. R.; Jiao, H. J. Am. Chem. Soc. 1997, 119, 6951 - 6952. (c) Apeloig, Y. in The Chemistry of Organosilicon Compounds (Eds.: Patai, S. and Rappoport, Z.), Wiley, New York, 1989, pp. 151 - 166 (d) Brook, A. G.; Brook, M. A. Adv. Organomet. Chem. 1996, 39, 71 - 158. (e) Schleyer, P. v. R.; Jiao, H.; Hommes, N. J. R. v. E.; Malkin, V. G.; Malkina, O. L. J. Am. Chem. Soc. 1997, 119, 12669 - 12670. (f) Apeloig, Y.; Karni, M. in The Chemistry of Organosilicon Compounds, Part II (Eds.: Apeloig, Y. and Rappoport, Z.), Wiley, New York, 1998, chap. 1. (f) Baldridge, K. K.; Uzan, O.; Martin, J. M. L. Organometallics 2000, 19, 1477 - 1487.

102. (a) Solouki, B.; Rosmus, P.; Bock, H.; Maier, G. Angew. Chem. 1980, 92, 56 - 57; Angew. Chem., Int. Ed. Engl. 1980, 19, 51 - 52. (b) Maier, G.; Mihm, G.; Reisenauer, H. P. Angew. Chem. 1980, 92, 58 - 59; Angew. Chem., Int. Ed. Engl. 1980, 19, 52 53. (c) Maier, G.; Mihm, G.; Reisenauer, H. P. Chem. Ber. 1982, 115, 801 - 803. (d) 
Maier, G.; Mihm, G.; Baumgärtner, R. O. W.; Reisenauer, H. P. Chem. Ber. 1984, 117, 2337 - 2350. (e) Jutzi, P.; Meyer, M.; Reisenauer, H. P.; Maier, G. Chem. Ber. 1989, $122,1227-1230$.

103. Märkl, G.; Schlosser, W. Angew. Chem. 1988, 100, 1009 - 1011; Angew. Chem., Int. Ed. Engl. 1988, 27, 963 - 965.

104. Wakita, K.; Tokitoh, N.; Okazaki, R.; Nagase, S. Angew. Chem. 2000, 112, 648 - 650; Angew. Chem., Int. Ed. 2000, 39, $634-636$.

105. Kabe, Y.; Ohkubo, K.; Ishikawa, H.; Ando, W. J. Am. Chem. Soc. 2000, 122, 3775 3776.

106. Abersfelder, K.; White, A. J. P.; Rzepa, H. S.; Scheschkewitz, D. Science 2010, 327, $564-566$.

107. Maier, G.; Schottler, K.; Reisenauer, H. P. Tetrahedron Lett. 1985, 26, 4079 - 4082.

108. (a) Rich, J. D.; West, R. J. Am. Chem. Soc. 1982, 104, 6884 - 6886. (b) Welsch, K. M.; Rich, J. D.; West, R. J. Organomet. Chem. 1987, 325, 105 - 115. (c) Sekiguchi, A.; Gillette, G. R.; West, R. Organometallics 1988, 7, 1226 - 1227. (d) Oikawa, T.; Nakata, N.; Matsumoto, T.; Kabe, Y. Sekiguchi, A. Heteroatom. Chem. 2008, 19, 87 $-92$.

109. For recent reviews see: (a) Lee, V. Y.; Sekiguchi, A.; Ichinohe, M.; Fukaya, N. J. Organomet. Chem. 2000, 611, 228 - 235. (b) Sekiguchi, A.; Lee, V. Y. Chem. Rev. 2003, 103, 1429 - 1447. (c) Tokitoh, N. Acc. Chem. Res. 2004, 37, 86 - 94. (d) Tokitoh, N. Bull. Chem. Soc. Jpn. 2004, 77, 429 - 441. (e) Lee, V. Y.; Sekiguchi, A. Angew. Chem. 2007, 119, 6716 - 6740; Angew. Chem. Int., Ed., 2007, 46, 6596 6620.

110. Takagi, N.; Shimizu, T.; Frenking, G. Chem. Eur. J. 2009, 15, 8593 - 8604.

111. Scheschkewitz, D.; Amii, H.; Gornitzka, H.; Schoeller, W. W.; Bourissou, D.; Bertrand, G. Science 2002, 295, 1880 - 1881. 
112. Jung, Y.; Head-Gordon, M. ChemPhysChem 2003, 4, 522 - 525.

113. (a) Schleyer, P. v. R.; Maerker, C.; Dransfeld, A.; Jiao, H.; Hommes, N. J. R. v. E. J. Am. Chem. Soc. 1996, 118, 6317 - 6318. (b) Chen, Z.; Wannere, C. S.; Corminboeuf, C.; Puchta, R.; Schleyer, P. v. R. Chem. Rev. 2005, 105, 3842 - 3888.

114. (a) The N-N bond length in $\left(\mathrm{H}_{3} \mathrm{Si}\right)_{2} \mathrm{NN}\left(\mathrm{SiH}_{3}\right)_{2}$ is $1.46 \AA$ : Glidewell, C.; Rankin, D. W. H.; Robiette, A. G.; Sheldrick, G. M. J. Chem. Soc. A. 1970, 318 - 320. (b) Veith, M.; Rammo, A. Z. Anorg. Allg. Chem. 2001, 627, $662-668$.

115. Cui, C.; Olmstead, M. M.; Fettinger, J. C.; Spikes, G. H.; Power, P. P. J. Am. Chem. Soc. 2005, 127, $17530-17541$.

116. (a) Pearson, R. J. Science 1966, 151, 172 - 177. (b) Pearson, R. J. J. Am. Chem. Soc. 1963, $85,3533-3539$.

117. (a) Pineda, L. W.; Jancik, V.; Colunga-Valladares, J. F.; Roesky, H. W.; Hofmeister, A.; Magull, J. Organometallics, 2006, 25, 2381 - 2383. (b) Saur, I.; Rima, G.; Miqueu, K.; Gornitzka, H.; Barrau, J. J. Organomet. Chem. 2003, 672, 77 - 85. (c) Veith, M.; Becker, S.; Huch, V. Angew. Chem. 1990, 102, 186 - 188; Angew. Chem., Int. Ed. Engl. 1990, 29, 216 - 218.

118. Barrau, J.; Rima, G. Coord. Chem. Rev. 1998, 178-180, 593 - 622.

119. (a) Jutzi, P.; Hoffmann, H. J.; Brauer, D. J.; Krüger, C. Angew. Chem. 1973, 85, 1116 - 1117; Angew. Chem., Int. Ed. Engl. 1973, 12, 1002 - 1003. (b) Stobart, S. R. J. Chem. Soc., Chem. Commun. 1979, 911 - 912.

120. Herrmann, W. A.; Denk, M.; Behm, J.; Scherer, W.; Klingan, F.-R.; Bock, H.; Solouki, B.; Wagner, M. Angew. Chem. 1992, 104, 1489 - 1492; Angew. Chem., Int. Ed. Engl. 1992, 31, $1485-1488$.

121. (a) Dias, H. V. R.; Wang, Z. J. Am. Chem. Soc. 1997, 119, 4650 - 4655. (b) Barrau, J.; Rima, G.; Amraoui, T. E. Organometallics 1998, 17, 607 - 614. (c) Ayers, A. E.; Marynick, D. S.; Dias, H. V. R. Inorg. Chem. 2000, 39, 4147 - 4151. (d) Zemlyansky, 
N. N.; Borisova, I. V.; Kuznetsova, M. G.; Khrustalev, V. N.; Ustynyuk, Y. A.; Nechaev, M. S.; Lunin, V. V.; Barrau, J.; Rima, G. Organometallics 2003, 22, 1675 1681. (e) Driess, M.; Dona, N.; Merz, K. Dalton Trans. 2004, 3176 - 3177. (f) Khrustalev, V. N.; Portnyagin, I. A.; Zemlyansky, N. N.; Borisova, I. V.; Ustynyuk, Y. A.; Yu. Antipin, M. J. Organomet. Chem. 2005, 690, 1056 - 1062. (g) Khrustalev, V. N.; Portnyagin, I. A.; Zemlyansky, N. N.; Borisova, I. V.; Nechaev, M. S.; Ustynyuk, Y. A.; Yu. Antipin, M.; Lunin, V. J. Organomet. Chem. 2005, 690, 1172 1177.

122. (a) Veith, M. Angew. Chem. 1987, 99, 1 - 14; Angew. Chem., Int. Ed. Engl. 1987, 26, 1 - 14. (b) Neumann, W. P. Chem. Rev. 1991, 91, 311 - 334. (c) Dias, H. V. R.; Wang, Z.; Jin, W. Coord. Chem. Rev. 1998, 176, 67 - 86. (d) Klinkhammer, K. W. in The Chemistry of Organic Germanium, Tin and Lead Compounds, Vol. 2; Rappoport, Z., Ed.; Wiley: New York, 2002; pp 284 - 332. (e) Kühl, O. Coord. Chem. Rev. 2004, 248, 411 - 427. (f) Saur, I.; Alonso, S. G.; Barrau, J. Appl. Organomet. Chem. 2005, 19, 414 - 428. (g) Leung, W.-P.; Kan, K.-W.; Chong, K.-H. Coord. Chem. Rev. 2007, $251,2253-2265$.

123. (a) Gans-Eichler, T.; Gudat, D.; Nieger, M. Angew. Chem. 2002, 114, 1966 - 1969; Angew. Chem., Int. Ed. 2002, 41, 1888 - 1892. (b) Manshell, S. M.; Russell, C. A.; Wass, D. F. Inorg. Chem. 2008, 47, 11367 - 11375.

124. (a) Lappert, M. F.; Power, P. P.; Sanger, A. R.; Srivastava, R. C. in Metal and Metalloid Amides, Ellis Horwood Ltd., Chichester, 1980. (b) Lappert, M. F. Main Group Metal Chemistry 1994, 17, 183 - 207. (c) Neumann, W. P. Chem. Rev. 1991, 91, 311- 334. (d) Weidenbruch, M. Eur. J. Inorg. Chem. 1999, 373 - 381. (e) Tokitoh, N.; Okazaki, R. Coord. Chem. Rev. 2000, 210, 251 - 277. (f) Boganov, S. E.; Egorov, M. V.; Faustov, V. I.; Nefedov, O. M. in The Chemistry of Organic Germanium, Tin 
and Lead Compounds; Rappoport, Z. Ed.; John Wiley and Sons: New York, 2002; Vol. 2, pp $749-839$.

125. Selected examples: (a) Bigwood, M. P.; Corvan, P. J.; Zuckerman, J. J. J. Am. Chem. Soc. 1981, 103, 7643 - 7646. (b) West, R. Science 1984, 225, 1109 - 1114. (c) Raabe, G.; Michl, J.; Chem. Rev. 1985, 85, 419 - 509. (d) Engelhardt, L. M.; Jolly, B. S.; Lappert, M. F.; Raston, C. L.; White, A. H. J. Chem. Soc. Chem. Commun. 1988, 336 - 337. (e) Jastrzebski, J. T. B. H.; van der Schaaf, P. A.; Boersma, J.; van Koten, G.; Zoutberg, M. C.; Heijdenrijk, D. Organometallics 1989, 8, 1373 - 1375. (f) Eaborn, C.; Izod, K.; Hitchcock, P. B.; Sözerli, S. E.; Smith, J. D. J. Chem. Soc. Chem. Commun. 1995, 1829 - 1830. (g) Baines, K. M.; Stibbs, W. G. Adv. Organomet. Chem. 1996, 39, 275 - 324. (h) Drost, C.; Hitchcock, P. B.; Lappert, M. F.; Pierssens, L. J. J. Chem. Soc., Chem. Commun. 1997, 1141 - 1142. (i) Hitchcock, P. B.; Lappert, M. F.; Layh, M. Inorg. Chim. Acta 1998, 269, 181 - 190. (j) Pu, L.; Olmstead, M. M.; Power, P. P.; Schiemenz, B. Organometallics 1998, 17, 5602 - 5206. (k) Ayers, A. E.; Marynick, D. S.; Dias, H. V. R. Inorg. Chem. 2000, 39, 4147 - 4151.

126. Holt, M. S.; Wilson, W. L.; Nelson, J. H. Chem. Rev. 1989, 89, 11 - 49.

127. Batchelor, R. J.; Ruddick, J. N. R.; Sams, J. R.; Aubke, F. Inorg. Chem. 1977, 16, $1414-1417$.

128. Hitchcock, P. B.; Lappert, M. F.; Lawless, G. A.; Lima, G. M.; Pierssens, L. J. J. Organomet. Chem. 2000, 601, $142-146$.

129. Jana, A.; Roesky, H. W.; Schulzke, C.; Döring, A.; Beck, T.; Pal, A.; Herbst-Irmer, R. Inorg Chem. 2009, 48, 193 - 197.

130. Brym, M.; Francis, M. D.; Jin, G.; Jones, C.; Mills, D. P.; Stasch, A. Organometallics 2006, $25,4799-4807$.

131. Nimitsiriwat, N.; Gibson, V. C.; Marshall, E. L.; White, A. J. P.; Dale, S. H.; Elsegood, M. R. J. Dalton Trans. 2007, $4464-4471$. 
132. Aubrecht, K. B.; Hillmyer, M. A.; Tolman, W. B. Macromolecules 2002, 35, 644 650.

133. (a) Harris, D. H.; Lappert, M. F.; Pedley, J. B.; Sharp, G. J. J. Chem. Soc. Dalton Trans. 1976, 945 - 949. (b) Gyenane, M. J. S.; Harris, D. H.; Lappert, M. F.; Power, P. P.; Rivière, P.; Rivière-Baudet, M. J. Chem. Soc. Dalton Trans. 1977, 2004 - 2009. (c) Jones, K.; Lappert, M. F. in 'Organotin Cornpounds', Ed. A. K. Sawyer, Marcel Dekker, New York, 1971, vol. 2, ch. 7. (d) Foley, S. R.; Zhou, Y.; Yap, G. P. A.; Richeson, D. S. Inorg. Chem. 2000, 39, 924 - 929. (e) Foley, S. R.; Yap, G. P. A.; Richeson, D. S. Organometallics 1999, 18, 4700 - 4705.

134. Pineda, L. W.; Jancik, V.; Starke, K.; Oswald, R. B.; Roesky, H. W. Angew. Chem. 2006, 118, 2664 - 2667; Angew. Chem., Int. Ed. 2006, 45, 2602 - 2605.

135. Jana, A.; Ghoshal, D.; Roesky, H. W.; Objartel, I.; Schwab, G.; Stalke, D. J. Am. Chem. Soc. 2009, 131, $1288-1293$.

136. Antolini, F.; Hitchcock, P. B.; Khvostov, A. V.; Lappert, M. F. Can. J. Chem. 2006, $84,269-276$.

137. Jana, A.; Sarish, S. P.; Roesky, H. W.; Schulzke, C.; Samuel, P. P. Chem Commun. 2010, $707-709$.

138. (a) Fischer, E. O. Nobel Lecture, December 11, 1973, http://nobelprize.org/chemistry/laureates/1973/fischer-lecture.pdf; see also Fischer, E. O. Angew. Chem. 1974, 86, 651 - 682. (b) Schrock, R. R. Nobel Lecture, December 8, 2005, http://nobelprize.org/chemistry/laureates/2005/schrock-lecture.pdf; see also Schrock, R. R. Angew. Chem. 2006, 118, 3832 - 3844; Angew. Chem., Int. Ed. 2006, $45,3748-3759$.

139. (a) Cadenbach, T.; Gemel, C.; Fischer, R. A. Angew. Chem. 2008, 120, 9286 - 9289; Angew. Chem., Int. Ed. 2008, 47, 9146 - 9149. (b) Cadenbach, T.; Bollermann, T.; Gemel, C.; Fernandez, I.; Von Hopffgarten, M.; Frenking, G.; Fischer, R. A. Angew. 
Chem. 2008, 120, 9290 - 9295; Angew. Chem., Int. Ed. 2008, 47, 9150 - 9154. (c) Schulz, S. Chem. Eur. J. 2010, 16, $6416-6428$.

140. Alcarazo, M.; Lehmann, C. W.; Anoop, A.; Thiel, W.; Fürstner, A. Nature Chem. 2009, $1,295-301$.

141. Bruker, SAINT v 7.68A., Bruker AXS Inc., Madison (WI, USA), 2009.

142. Sheldrick, G. M. SADABS 2008/2, Göttingen, 2008.

143. SAINT-NT, Bruker AXS Inc., Madison, Wisconsin (USA) 2000.

144. Sheldrick, G. M. SADABS 2.0, Universität Göttingen, Germany, 2000. 


\section{List of Scientific Contributions}

\section{$\underline{\text { Publications }}$}

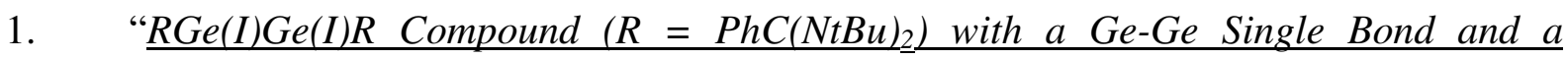
Comparison with the Gauche Conformation of Hydrazine"

Selvarajan Nagendran, Sakya S. Sen, Herbert W. Roesky, Debasis Koley, Helmut Grubmüller, Aritra Pal, Regine Herbst-Irmer, Organometallics 2008, 27, 5459 - 5463.

2. "End-On Nitrogen Insertion of a Diazo Compound into a Germanium(II) Hydrogen Bond and a Comparable Reaction with Diethyl Azodicarboxylate"

Anukul Jana, Sakya S. Sen, Herbert W. Roesky, Carola Schulzke, Sudipta Dutta, Swapan K. Pati, Angew. Chem. 2009, 121, 4310 - 4312; Angew. Chem. Int. Ed. 2009, $48,4246-4248$.

3. "A Remarkable Base-Stabilized Bis(silylene) with a Silicon(I)-Silicon(I) Bond"

Sakya S. Sen, Anukul Jana, Herbert W. Roesky, Carola Schulzke, Angew. Chem. 2009, 121, 8688 - 8690; Angew. Chem. Int. Ed. 2009, 48, 8536 - 8538.

4. "Neutral Penta- and Hexacoordinate N-Heterocyclic Carbene Complexes Derived from $\left.\operatorname{SiX}_{4} \underline{ }_{(X=F, B r}\right)^{\prime \prime}$

Rajendra S. Ghadwal, Sakya S. Sen, Herbert W. Roesky, Gasper Tavcar, Sebastian Merkel, Dietmar Stalke, Organometallics 2009, 28, 6374 - 6377.

5. "High Yield Access to Silylene RSiCl $\left(R=P h C(N t B u)_{2}\right)$ and its Reactivity toward Alkyne: Synthesis of Stable Disilacyclobutene"

Sakya S. Sen, Herbert W. Roesky, Daniel Stern, Julian Henn, Dietmar Stalke, J. Am. Chem. Soc. 2010, 132, 1123 - 1126.

6. "Convenient Access to Monosilicon Epoxides with Pentacoordinate silicon"

Rajendra S. Ghadwal, Sakya S. Sen, Herbert W. Roesky, Markus Granitzka, Daniel Kratzert, Sebastian Merkel, Dietmar Stalke, Angew. Chem. 2010, 122, 4044 - 4047; Angew. Chem. Int. Ed. 2010, 49, 3952 - 3955.

7. "Synthesis of a Stable Four-membered $\mathrm{Si}_{2} \underline{O}_{2}$ Ring and a Dimer with Two Fourmembered $\mathrm{Si}_{2} \underline{\mathrm{O}}_{2}$ Rings bridged by Two Oxygen Atoms, with Five-coordinate Silicon atoms in both the Ring Systems"

Sakya S. Sen, Gašper Tavčar, Herbert W. Roesky, Daniel Kratzert, Jakob Hey, Dietmar Stalke, Organometallics 2010, 29, 2343 - 2347. 
8. "Reactivity Studies of a Ge(I)-Ge(I) Compound with and without cleavage of the GeGe bond"

Sakya S. Sen, Daniel Kratzert, Daniel Stern, Herbert W. Roesky, Dietmar Stalke, Inorg. Chem. 2010, 49, $5786-5788$.

9. "Synthesis, Structure, and Theoretical Investigation of Amidinato Supported 1,4disilabenzene"

Sakya S. Sen, Herbert W. Roesky, Kathrin Meindl, Daniel Stern, Julian Henn, A. Claudia Stückl, Dietmar Stalke, Chem. Commun. 2010, 46, 5873 - 5875 (Selected as a HOT article).

10. "Reaction of a Bis-silylene ( $\mathrm{LSi}$-SiL, L $\left.=\mathrm{PhC}(\mathrm{NtBu})_{2}\right)$ and a Heteroleptic Chloro Silylene (LSiCl) with Benzil: Formation of Bis(siladioxolene) and Monosiladioxolene Analogue with Five-Coordinate Silicon Atoms in Both Ring Systems"

Gašper Tavčar, Sakya S. Sen, Herbert W. Roesky, Jakob Hey, Daniel Kratzert, Dietmar Stalke, Organometallics 2010, 29, 3930 - 3935.

11. "Synthesis of Monomeric Divalent Tin(II) Compounds with terminal Chloride, Amide, and Ttriflate substituents"

Sakya S. Sen, Malte P. Kritzler-Kosch, Selvarajan Nagendran, Herbert W. Roesky, Tobias Beck, Aritra Pal, Regine Herbst-Irmer, Eur. J. Inorg. Chem. 2010, 33, 5304 5311.

12. "One pot Synthesis of tricylodisilaheptene Analogue and Jutzi's Disilene"

Shabana Khan, Sakya S. Sen, Daniel Kratzert, Reent Michel, Herbert W. Roesky, Dietmar Stalke, Inorg. Chem. 2010, 49, 9689 - 9693.

13. "Facile Syntheses of Silylene Nickel Carbonyl Complexes from Lewis Base Stabilized Chlorosilylenes"

Gašper Tavčar, Sakya S. Sen, Ramachandran Azhakar, Andrea Thorn, Herbert W. Roesky, Inorg. Chem. 2010, 49, 10199 - 10202.

14 " $\not$-Diketiminate Stabilized Magnesium Hydroxide, Heterobimetallic, and Halide Complexes: Synthesis and X-ray Structural Studies"

Sharanappa Nembenna, Sanjay Singh, Sakya S. Sen, Herbert W. Roesky, Holger Ott, Dietmar Stalke, ZAAC 2010, DOI: 10.1002/zaac.201000348. 
15. "Stable Cyclobutadiene Analogues with three and four Heteroatoms in the fourmembered Ring"

Sakya S. Sen, Shabana Khan, Herbert W. Roesky, Daniel Kratzert, Kathrin Meindl, Julian Henn, Dietmar Stalke, Angew. Chem. 2010, DOI: 10.1002/ange.201005866.; Angew. Chem. Int. Ed. 2010, DOI: 10.1002/anie.201005866.

16. "Synthesis, Characterization, and Structure of $\left[\left\{P h C(N t B u)_{2} L_{2} \underline{G e}_{2} \underline{e}_{2}(\mu-S)_{2} \underline{C l_{2}} \underline{L}_{2}\right.\right.$ and a Germadithiocarboxylate Analogue"

Sakya S. Sen, Rajendra S. Ghadwal, Daniel Kratzert, Daniel Stern, Herbert W. Roesky, Dietmar Stalke, Organometallics 2010 (Accpeted article).

17. "Synthesis and Characterization of Stable Silicon Heterocycles by Oxidative Addition of Organic Substrates with Chloro Silylene [PhC $(\mathrm{NtBu})_{2}$ SiCll]"

Shabana Khan, Sakya S. Sen, Daniel Kratzert, Gašper Tavčar, Herbert W. Roesky, Dietmar Stalke, Chem. Eur. J. 2010 (Accepted article).

\section{Presentations}

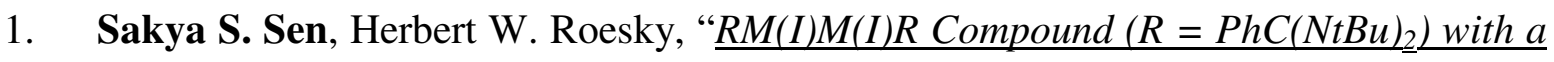
$\underline{M-M}$ Single Bond" in Göttinger Chemieforum, Göttingen, Germany, July 2009. (Awarded as Best Poster Prize)

2. Herbert W. Roesky, Rajendra S. Ghadwal, Anukul Jana, Sakya S. Sen, " of Compounds with Low Valent Elements of Group 14"; Abstracts of Papers, 19th International Symposium on Fluorine Chemistry, Jackson Hole, Wyoming, USA, 23-28 ${ }^{\text {th }}$ August, 2009

3. Sakya S. Sen, Herbert W. Roesky, "Synthesis and Characterization of a New Class of Lanthanide Compounds Containing a Ln-O-M Arrangement and a Novel Chlorine Centered Lanthanide Cluster" in SPP 1166 Molekülchemie / Katalyse, Karlsruhe, Germany, April 2010. (Oral Presentation)

4. Gašper Tavčar, Sakya S. Sen, Jakob Hey, Herbert W. Roesky, "Reactivity of some silicon compounds in low oxidation states" in Slovenski Kemijski Dnevi, Maribor, Slovenia, 23-24 ${ }^{\text {th }}$ September, 2010.

5. Sakya S. Sen, Herbert W. Roesky, "New Class of Lanthanide Compounds Containing a Ln-O-M Arrangement and Novel Chlorine Centered Lanthanide Clusters" in Terrae Rarae 2010, Bayreuth, 6- $8^{\text {th }}$ October, 2010. (Poster Presentation) 


\section{Lebenslauf}

Name Sakya Singha Sen

Geboren $\quad 2^{\text {nd }}$ March, 1983 in Kolkata

Staatsangehörigkeit indisch

Schulbesuch

$1989-1999 \quad$ Secondary School (Class I - X)

$1999-2001 \quad$ Higher Secondary (Class XI - XII)

Studium

$2001-2004 \quad$ B.Sc. in Chemie, University of Kolkata, India

2004 - 2006 M.Sc. in Chemie, IIT Kharagpur, India

Promotion

2006 November - present Experimentelle Promotionsarbeit im Institut für Anorganische Chemie Göttingen unter der Leitung von Prof. Dr. Dr. h. c. mult. Herbert W. Roesky

Promotionsarbeit mit dem Titel "Preparation and Reactions of Compounds with Heavier Group 14 Elements in Low Oxidation States" 